\title{
Evaluation of the Start- Up Core Physics Tests at Japan's High Temperature Engineering Test Reactor (Annular Core Loadings)
}

John D. Bess

Nozomu Fujimoto James W. Sterbentz Luka Snoj Atsushi Zukeran

March 2010

The INL is a U.S. Department of Energy National Laboratory operated by Battelle Energy Alliance

Idaho National Laboratory 
INL/EXT-09-15794

\title{
Evaluation of the Start-Up Core Physics Tests at Japan's High Temperature Engineering Test Reactor (Annular Core Loadings)
}

\author{
John D. Bess \\ Nozomu Fujimoto ${ }^{1}$ \\ James W. Sterbentz \\ Luka Snoj $^{2}$ \\ Atsushi Zukeran ${ }^{3}$ \\ ${ }^{1}$ Japan Atomic Energy Agency \\ ${ }^{2}$ Jozef Stefan Institute \\ ${ }^{3}$ Senior Reactor Physics Consultant
}

March 2010

\begin{abstract}
Idaho National Laboratory
Idaho Falls, Idaho 83415
\end{abstract}

http://www.inl.gov

Prepared for the

U.S. Department of Energy

Office of Nuclear Energy

Under DOE Idaho Operations Office

Contract DE-AC07-05ID14517 


\title{
EVALUATION OF THE START-UP CORE PHYSICS TESTS AT JAPAN'S HIGH TEMPERATURE ENGINEERING TEST REACTOR (ANNULAR CORE LOADINGS)
}

\author{
Evaluators \\ John D. Bess \\ Idaho National Laboratory \\ Nozomu Fujimoto \\ Japan Atomic Energy Agency \\ Internal Reviewers \\ James W. Sterbentz \\ Idaho National Laboratory
}

Independent Reviewers

Luka Snoj

Jozef Stefan Institute

Atsushi Zukeran

Senior Reactor Physics Consultant 
NEA/NSC/DOC(2006)1

Gas Cooled (Thermal) Reactor - GCR

HTTR-GCR-RESR-002

CRIT-REAC-RRATE

Status of Compilation / Evaluation / Peer Review

\begin{tabular}{|c|c|c|c|c|c|}
\hline & Section 1 & Compiled & $\begin{array}{l}\text { Independent } \\
\text { Review }\end{array}$ & $\begin{array}{l}\text { Working Group } \\
\text { Review }\end{array}$ & Approved \\
\hline 1.0 & " DETAILED DESCRIPTION & YES & YES & YES & YES \\
\hline 1.1 & $\begin{array}{l}\text { Description of the Critical and / or } \\
\text { Subcritical Configuration }\end{array}$ & YES & YES & YES & YES \\
\hline 1.2 & $\begin{array}{l}\text { Description of Buckling and } \\
\text { Extrapolation Length Measurements }\end{array}$ & NA & NA & NA & NA \\
\hline 1.3 & $\begin{array}{l}\text { Description of Spectral Characteristics } \\
\text { Measurements }\end{array}$ & NA & NA & NA & NA \\
\hline 1.4 & $\begin{array}{l}\text { Description of Reactivity Effects } \\
\text { Measurements }\end{array}$ & YES & YES & YES & YES \\
\hline 1.5 & $\begin{array}{l}\text { Description of Reactivity Coefficient } \\
\text { Measurements }\end{array}$ & NA & NA & NA & NA \\
\hline 1.6 & Description of Kinetics Measurements & NA & NA & NA & NA \\
\hline 1.7 & $\begin{array}{l}\text { Description of Reaction-Rate } \\
\text { Distribution Measurements }\end{array}$ & YES & YES & YES & YES \\
\hline 1.8 & $\begin{array}{l}\text { Description of Power Distribution } \\
\text { Measurements }\end{array}$ & NA & NA & NA & NA \\
\hline 1.9 & Description of Isotopic Measurements & NA & NA & NA & NA \\
\hline 1.10 & $\begin{array}{l}\text { Description of Other Miscellaneous } \\
\text { Types of Measurements }\end{array}$ & NA & NA & NA & NA \\
\hline & Section 2 & Evaluated & $\begin{array}{c}\text { Independent } \\
\text { Review }\end{array}$ & $\begin{array}{c}\text { Working Group } \\
\text { Review }\end{array}$ & Approved \\
\hline 2.0 & $\begin{array}{l}\text { EVALUATION OF EXPERIMENTAL } \\
\text { DATA }\end{array}$ & YES & YES & YES & YES \\
\hline 2.1 & $\begin{array}{l}\text { Evaluation of Critical and / or Subcritical } \\
\text { Configuration Data }\end{array}$ & YES & YES & YES & YES \\
\hline 2.2 & $\begin{array}{l}\text { Evaluation of Buckling and Extrapolation } \\
\text { Length Data }\end{array}$ & NA & NA & NA & NA \\
\hline 2.3 & $\begin{array}{l}\text { Evaluation of Spectral Characteristics } \\
\text { Data }\end{array}$ & NA & NA & NA & NA \\
\hline 2.4 & Evaluation of Reactivity Effects Data & YES & YES & YES & YES \\
\hline 2.5 & Evaluation of Reactivity Coefficient Data & NA & NA & NA & NA \\
\hline 2.6 & $\begin{array}{l}\text { Evaluation of Kinetics Measurements } \\
\text { Data }\end{array}$ & NA & NA & NA & NA \\
\hline 2.7 & $\begin{array}{l}\text { Evaluation of Reaction Rate } \\
\text { Distributions }\end{array}$ & YES & YES & YES & YES \\
\hline 2.8 & Evaluation of Power Distribution Data & NA & NA & NA & NA \\
\hline 2.9 & Evaluation of Isotopic Measurements & NA & NA & NA & NA \\
\hline 2.10 & $\begin{array}{l}\text { Evaluation of Other Miscellaneous Types } \\
\text { of Measurements }\end{array}$ & NA & NA & NA & NA \\
\hline
\end{tabular}


NEA/NSC/DOC(2006)1

Gas Cooled (Thermal) Reactor - GCR

HTTR-GCR-RESR-002

CRIT-REAC-RRATE

\begin{tabular}{|c|c|c|c|c|}
\hline Section 3 & Compiled & $\begin{array}{c}\text { Independent } \\
\text { Review }\end{array}$ & $\begin{array}{c}\text { Working } \\
\text { Group Review } \\
\end{array}$ & Approved \\
\hline $\begin{array}{ll}3.0 & \text { BENCHMARK SPECIFICATIONS } \\
\end{array}$ & YES & YES & YES & YES \\
\hline $\begin{array}{ll}3.1 & \text { Benchmark-Model Specifications for } \\
\text { Critical and / or Subcritical } \\
\text { Measurements }\end{array}$ & YES & YES & YES & YES \\
\hline $\begin{array}{ll}3.2 & \text { Benchmark-Model Specifications for } \\
& \text { Buckling and Extrapolation Length } \\
& \text { Measurements } \\
\end{array}$ & NA & NA & NA & NA \\
\hline $\begin{array}{ll}3.3 & \text { Benchmark-Model Specifications for } \\
& \text { Spectral Characteristics Measurements }\end{array}$ & NA & NA & NA & NA \\
\hline $\begin{array}{ll}3.4 & \text { Benchmark-Model Specifications for } \\
& \text { Reactivity Effects Measurements }\end{array}$ & YES & YES & YES & YES \\
\hline $\begin{array}{ll}3.5 & \text { Benchmark-Model Specifications for } \\
& \text { Reactivity Coefficient Measurements }\end{array}$ & NA & NA & NA & NA \\
\hline $\begin{array}{ll}3.6 & \text { Benchmark-Model Specifications for } \\
& \text { Kinetics Measurements }\end{array}$ & NA & NA & NA & NA \\
\hline $\begin{array}{ll}3.7 & \text { Benchmark-Model Specifications for } \\
& \text { Reaction-Rate Distribution } \\
& \text { Measurements }\end{array}$ & YES & YES & YES & YES \\
\hline $\begin{array}{ll}3.8 & \text { Benchmark-Model Specifications for } \\
& \text { Power Distribution Measurements }\end{array}$ & NA & NA & NA & NA \\
\hline $\begin{array}{ll}3.9 & \text { Benchmark-Model Specifications for } \\
& \text { Isotopic Measurements }\end{array}$ & NA & NA & NA & NA \\
\hline $\begin{array}{l}3.10 \text { Benchmark-Model Specifications of } \\
\text { Other Miscellaneous Types of } \\
\text { Measurements }\end{array}$ & NA & NA & NA & NA \\
\hline Section 4 & Compiled & $\begin{array}{c}\text { Independent } \\
\text { Review }\end{array}$ & $\begin{array}{c}\text { Working } \\
\text { Group Review } \\
\end{array}$ & Approved \\
\hline $\begin{array}{ll}4.0 & \text { RESULTS OF SAMPLE } \\
& \text { CALCULATIONS }\end{array}$ & YES & YES & YES & YES \\
\hline $\begin{array}{ll}4.1 & \text { Results of Calculations of the Critical or } \\
\text { Subcritical Configurations }\end{array}$ & YES & YES & YES & YES \\
\hline $\begin{array}{ll}4.2 & \text { Results of Buckling and Extrapolation } \\
\text { Length Calculations }\end{array}$ & NA & NA & NA & NA \\
\hline $\begin{array}{ll}4.3 & \text { Results of Spectral Characteristics } \\
& \text { Calculations }\end{array}$ & NA & NA & NA & NA \\
\hline 4.4 Results of Reactivity Effect Calculations & YES & YES & YES & YES \\
\hline $\begin{array}{ll}4.5 & \text { Results of Reactivity Coefficient } \\
& \text { Calculations }\end{array}$ & NA & NA & NA & NA \\
\hline $\begin{array}{ll}\text { 4.6 } & \text { Results of Kinetics Parameter } \\
& \text { Calculations }\end{array}$ & NA & NA & NA & NA \\
\hline $\begin{array}{ll}\text { 4.7 Results of Reaction-Rate Distribution } \\
\text { Calculations }\end{array}$ & YES & YES & YES & YES \\
\hline $\begin{array}{ll}4.8 & \begin{array}{l}\text { Results of Power Distribution } \\
\text { Calculations }\end{array} \\
\end{array}$ & NA & NA & NA & NA \\
\hline 4.9 Results of Isotopic Calculations & NA & NA & NA & NA \\
\hline $\begin{array}{l}\text { 4.10 Results of Calculations of Other } \\
\text { Miscellaneous Types of Measurements }\end{array}$ & NA & NA & NA & NA \\
\hline Section 5 & Compiled & $\begin{array}{c}\text { Independent } \\
\text { Review }\end{array}$ & $\begin{array}{c}\text { Working Group } \\
\text { Review }\end{array}$ & Approved \\
\hline $\begin{array}{lll}5.0 & \text { REFERENCES } \\
\end{array}$ & YES & YES & YES & YES \\
\hline $\begin{array}{l}\text { Appendix A: Computer Codes, Cross Sections, and } \\
\text { Typical Input Listings }\end{array}$ & YES & YES & YES & YES \\
\hline
\end{tabular}


NEA/NSC/DOC(2006)1

Gas Cooled (Thermal) Reactor - GCR

HTTR-GCR-RESR-002

CRIT-REAC-RRATE

\title{
EVALUATION OF THE START-UP CORE PHYSICS TESTS AT JAPAN'S HIGH TEMPERATURE ENGINEERING TEST REACTOR (ANNULAR CORE LOADINGS)
}

\author{
IDENTIFICATION NUMBER: HTTR-GCR-RESR-002 \\ CRIT-REAC-RRATE
}

\begin{abstract}
KEY WORDS: annular cores, critical configurations, graphite-moderated, graphite-reflected, helium coolant, HTTR, low enriched uranium (LEU), pin-in-block fuel, prismatic fuel, thermal reactor, TRISO, uranium oxide fuel
\end{abstract}

\section{SUMMARY INFORMATION}

\subsection{DETAILED DESCRIPTION}

The High Temperature Engineering Test Reactor (HTTR) of the Japan Atomic Energy Agency (JAEA) is a $30 \mathrm{MWth}$, graphite-moderated, helium-cooled reactor that was constructed with the objectives to establish and upgrade the technological basis for advanced high-temperature gas-cooled reactors (HTGRs) as well as to conduct various irradiation tests for innovative high-temperature research. The core size of the HTTR represents about one-half of that of future HTGRs, and the high excess reactivity of the HTTR, necessary for compensation of temperature, xenon, and burnup effects during power operations, is similar to that of future HTGRs. During the start-up core physics tests of the HTTR, various annular cores were formed to provide experimental data for verification of design codes for future HTGRs (Ref. 1, p. 310).

The Japanese government approved construction of the HTTR in the 1989 fiscal year budget; construction began at the Oarai Research and Development Center in March 1991 and was completed May 1996. Fuel loading began July 1, 1998, from the core periphery. The first criticality was attained with an annular core on November 10, 1998 at 14:18, followed by a series of start-up core physics tests (Figure 1.1) until a fully-loaded core was developed on December 16, 1998. Criticality tests were carried out into January 1999. The first full power operation with an average core outlet temperature of $850^{\circ} \mathrm{C}$ was completed on December 7, 2001, and operational licensing of the HTTR was approved on March 6, 2002. The HTTR attained high temperature operation at $950{ }^{\circ} \mathrm{C}$ in April 19, 2004. After a series of safety demonstration tests, it will be used as the heat source in a hydrogen production system by 2015 (Ref. 3, pp. 12-14).

Hot zero-power critical, ${ }^{\mathrm{a}}$ rise-to-power, ${ }^{\mathrm{b}}$ irradiation, ${ }^{\mathrm{c}}$ and safety demonstration testing ${ }^{\mathrm{de}}$ have also been performed with the HTTR, representing additional means for computational validation efforts. Power tests were performed in steps from 0 to $30 \mathrm{MW}$, with various tests performed at each step to confirm core

\footnotetext{
a J. C. Kuijper, X. Raepsaet, J. B. M. de Haas, W. von Lensa, U. Ohlig, H-J. Ruetten, H. Brockmann, F. Damian, F. Dolci, W. Bernnat, J. Oppe, J. L. Kloosterman, N. Cerullo, G. Lomonaco, A. Negrini, J. Magill, and R. Seiler, "HTGR Reactor Physics and Fuel Cycle Studies,” Nucl. Eng. Des., 236: 615-634 (2006).

b S. Nakagawa, Y. Tachibana, K. Takamatsu, S. Ueta, and S. Hanawa, "Performance Test of HTTR,” Nucl. Eng. Des., 233: 291-300 (2004).

${ }^{c}$ T. Shibata, T. Kikuchi, S. Miyamoto, and K. Ogura, “Assessment of Irradiation Temperature Stability of the First Irradiation Test Rig in the HTTR,” Nucl. Eng. Des., 223: 133-143 (2003).

${ }^{\text {d }}$ Y. Tachibana, S. Nakagawa, T. Takeda, A. Saikusa, T. Furusawa, K. Takamatsu, K. Sawa, and T. Iyoku, "Plan for the First Phase of Safety Demonstration Tests of the High Temperature Engineering Test Reactor (HTTR)," Nucl. Eng. Des., 224: 179-197 (2003).

e S. Nakagawa, K. Takamatsu, Y. Tachibana, N. Sakaba, and T. Iyoku, "Safety Demonstration Tests using High Temperature Engineering Test Reactor,” Nucl. Eng. Des., 233: 301-308 (2004).
} 
NEA/NSC/DOC(2006)1

\section{Gas Cooled (Thermal) Reactor - GCR \\ HTTR-GCR-RESR-002 \\ CRIT-REAC-RRATE}

characteristics, thermal-hydraulic properties, and radiation shielding (Ref. 4, p. 284). The hightemperature test operation at $950{ }^{\circ} \mathrm{C}$ represented the fifth and final phase of the rise-to-power tests. ${ }^{\mathrm{a}}$ The safety tests demonstrated inherent safety features of the HTTR such as slow temperature response during abnormal events due to the large heat capacity of the core and the negative reactivity feedback. ${ }^{b}$

The experimental benchmark performed and currently evaluated in this report pertains to the data available for the annular core criticals from the initial six isothermal, annular and fully-loaded, core critical measurements performed at the HTTR. Evaluation of the start-up core physics tests specific to the fully-loaded core is compiled elsewhere (HTTR-GCR-RESR-001).

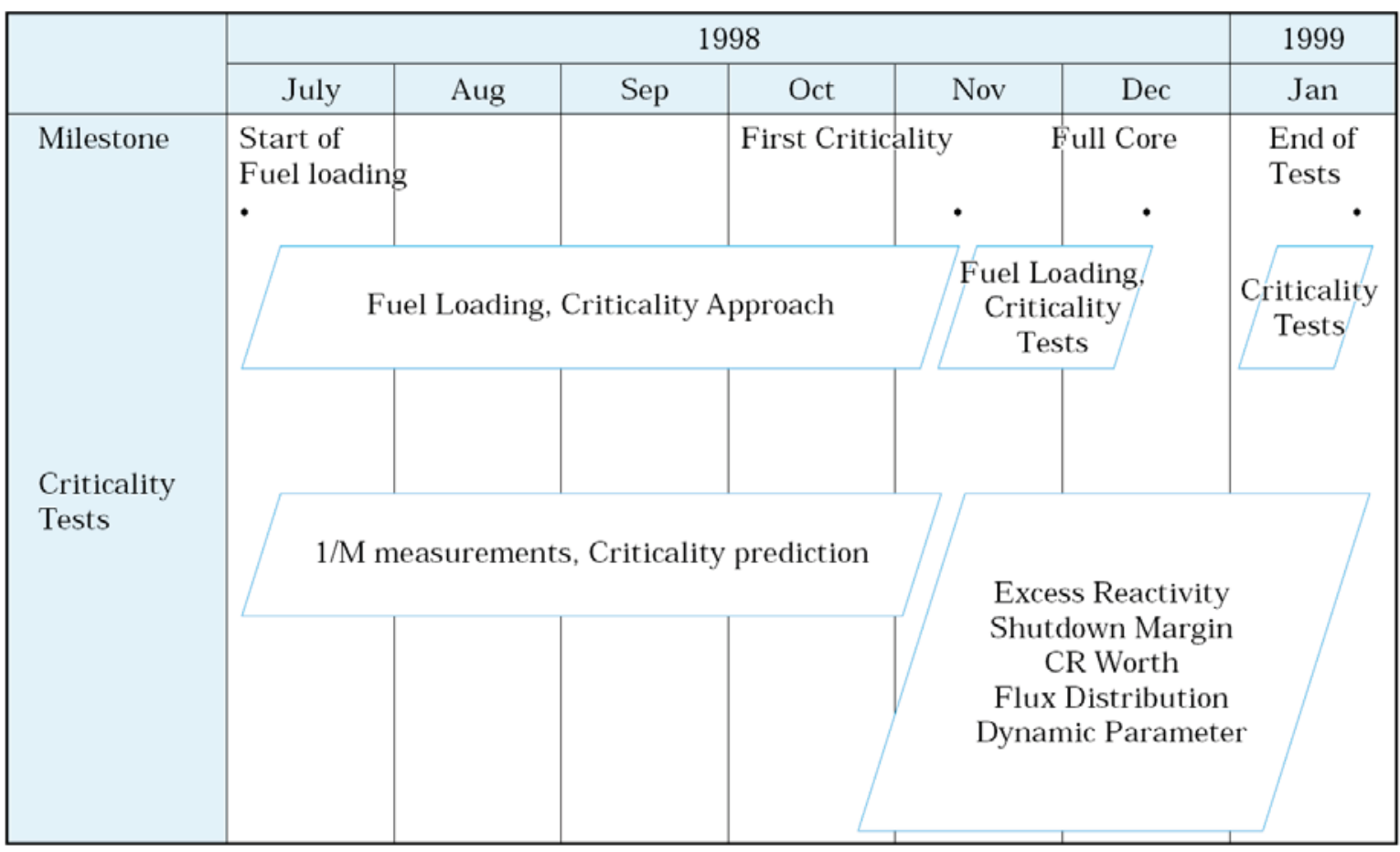

Figure 1.1. Progress of Start-Up Core Physics Tests. ${ }^{c}$

\subsection{Description of the Critical and / or Subcritical Configuration}

\subsubsection{Overview of Experiment}

The initial start-up core physics tests for the High Temperature Engineering Test Reactor were performed between the months of July 1998 and January 1999. The HTTR facility is at the Oarai Research and Development Center of the JAEA.

\footnotetext{
${ }^{a}$ S. Fujikawa, H. Hayashi, T. Nakazawa, K. Kawasaki, T. Iyoku, S. Nakagawa, and N. Sakaba, "Achievement of Reactor-Outlet Coolant Temperature of $950^{\circ} \mathrm{C}$ in HTTR,” J. Nucl. Sci. Tech., 41(12): 1245-1254 (December 2004).

${ }^{\mathrm{b}}$ S. Nakagawa, D. Tochio, K. Takamatsu, M. Goro, and T. Takeda, "Improvement of Analysis Technology for High Temperature Gas-Cooled Reactor by using Data Obtained in High Temperature Engineering Test Reactor," $J$. Power Energy Syst., 2(1): 83-91 (2008).

c "Present Status of HTGR Research and Development,” Japan Atomic Energy Research Institute, Tokaimura, March 2004.
}

Revision: 0 
NEA/NSC/DOC(2006)1

Gas Cooled (Thermal) Reactor - GCR

HTTR-GCR-RESR-002

CRIT-REAC-RRATE

First criticality was attained on November 10, 1998, and the core was fully loaded in December 1998. During the fuel loading period, various tests were conducted under cold clean conditions as the start-up core physics tests. During these tests, the power was limited to below $30 \mathrm{~W}$ and the helium pressure was 1 bar. The tests for the critical approach, excess reactivity, shutdown margin, neutron flux distribution and control rod worth were conducted (Ref. 4, p. 284). Elsewhere the pressure is listed as 1 atmosphere (Ref. 1, p. 312). One atmosphere is equal to $1.01325 \times 10^{5} \mathrm{~Pa}$, or $1.01325 \mathrm{bar}$.

Of the initial six isothermal, annular and fully-loaded, core critical measurements that were performed, only the annular core loadings were evaluated in this benchmark analysis. The maximum uncertainty range is between -1.03 and $+1.00 \% \Delta \mathrm{k}(1 \sigma)$ for the 19 -fuel-column core, with a decrease in the uncertainty as graphite dummy blocks are replaced by fuel blocks in the core. Dominant uncertainties are the impurities in the IG-110 graphite blocks, PGX graphite reflector blocks, and IG-11 graphite dummy blocks. Comprehensive biases could not be completed for all aspects of this experiment.

Currently the calculations performed using the benchmark models have a $\mathrm{k}_{\mathrm{eff}}$ between 1.4 to $2.7 \%$ of the experimental benchmark value and within $3 \sigma$, except for configuration 4 , which is within $4 \sigma$. It is currently difficult to obtain the necessary information to further improve the confidence in the benchmark model and effectively reduce the overall uncertainty; the necessary data is proprietary and its released is being restricted, because the benchmark configuration of the HTTR core is the same that is currently in operation. Once this information is made available, the HTTR benchmark can be adjusted as appropriate.

\subsubsection{Geometry of the Experiment Configuration and Measurement Procedure}

The geometric information publicly available for this benchmark can be found compiled in Section 1.1.2 of HTTR-GCR-RESR-001.

\subsubsection{Description of Criticality Measurements}

Before fuel loading, the whole region in the core was filled with graphite dummy blocks. Helium gas was filled up to $1 \mathrm{~atm}$ at room temperature. Dummy blocks were replaced with fuel blocks, column by column, in the fuel loading, starting from the core periphery to the center, as shown in Figure 1.2. 
HTTR-GCR-RESR-002

\section{CRIT-REAC-RRATE}

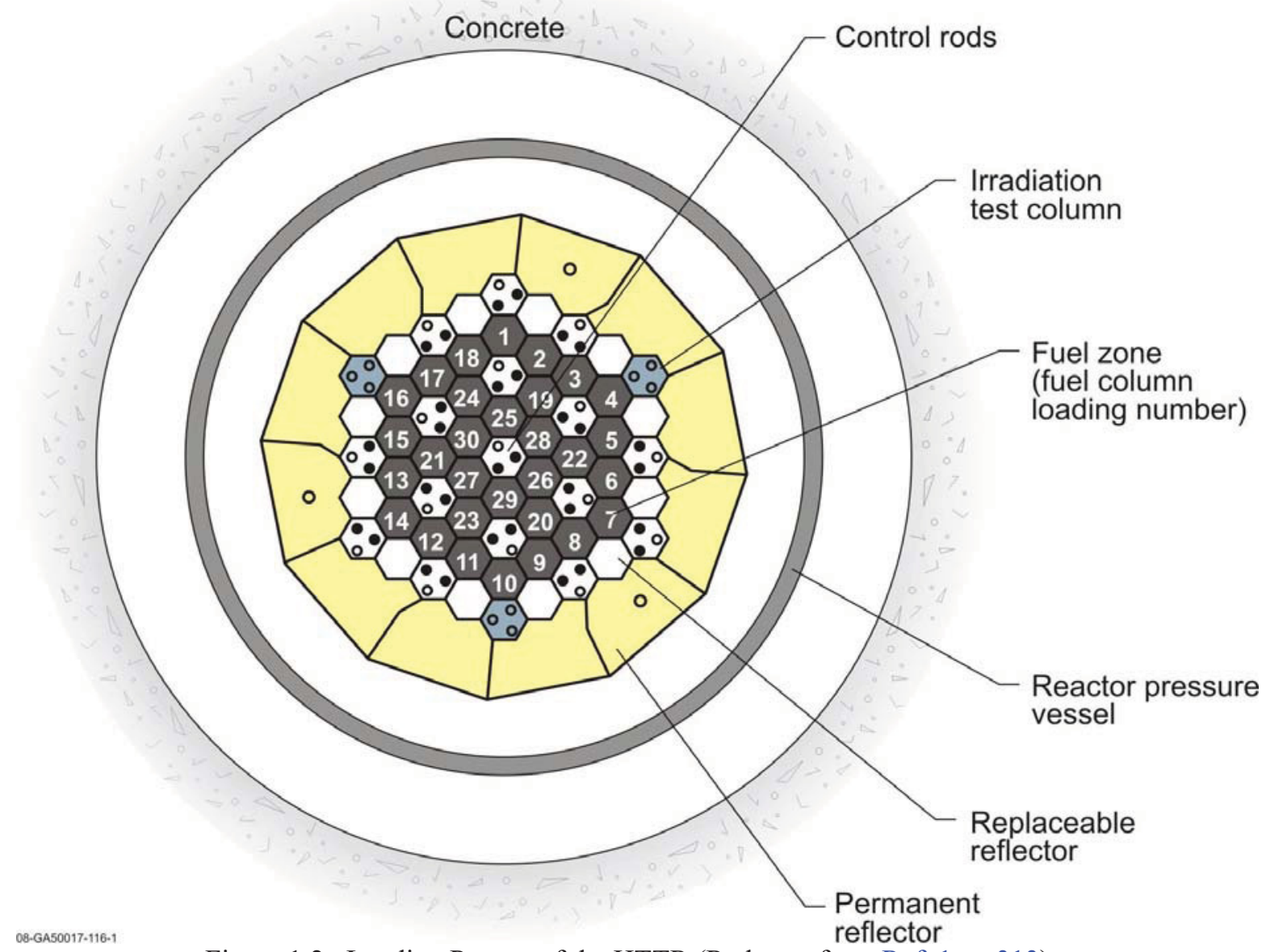

Figure 1.2. Loading Pattern of the HTTR (Redrawn from Ref. 1, p. 313).

In the first phase of fuel loading, six fuel columns were loaded consecutively. In the second phase, three fuel columns were loaded at a time, until a total of 15 columns were in the core. In the third phase of fuel loading, columns were loaded one at a time, so as to identify the number of fuel columns needed for the first criticality. In every loading phase, all control rods were withdrawn from the core after each fuel loading, and inverse multiplication factors were measured. Initial criticality was achieved with 19 fuel columns loaded. Symmetric annular cores were formed at 21-, 24-, and 27-fuel-column loaded cores. The fully-loaded core consists of 30 fuel columns. Figure 1.3 shows some of the fuel-loading steps during the initial core loading (Ref. 4, p. 285). 
NEA/NSC/DOC(2006)1

Gas Cooled (Thermal) Reactor - GCR

HTTR-GCR-RESR-002

CRIT-REAC-RRATE

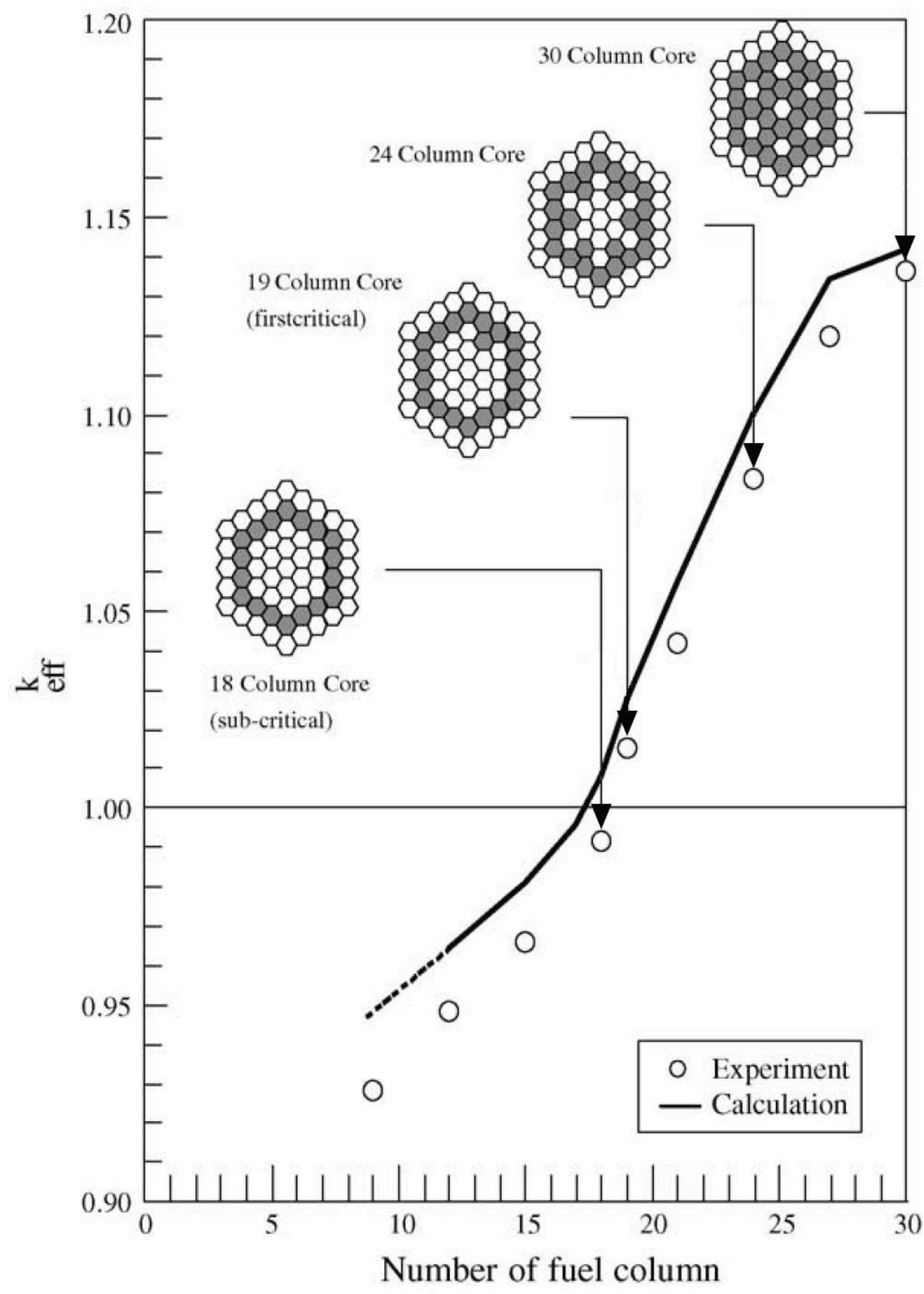

Figure 1.3. Critical Approach at Room Temperature using the Fuel Addition Method (Ref. 4, p. 285). 
NEA/NSC/DOC(2006)1

\section{Gas Cooled (Thermal) Reactor - GCR \\ HTTR-GCR-RESR-002 \\ CRIT-REAC-RRATE}

The inverse neutron multiplication factor was monitored in order to observe the approach to criticality; the core was regarded as critical when the neutron flux was self-sustained after removing the temporary neutron source. After the initial criticality, reactivity increase was measured using the IK (Inverse Kinetics) method. The excess reactivity of the core was then obtained by adding all increments of the reactivity from the first criticality to the fully-loaded core (Ref. 2, p. 14).

Inverse multiplication factors were evaluated at $0,6,9,12,15,16,17$, and 18 fuel-column-loaded cores to predict the first criticality (Ref. 2, p. 39). The inverse multiplication data were recorded and are shown in Figure 1.4.

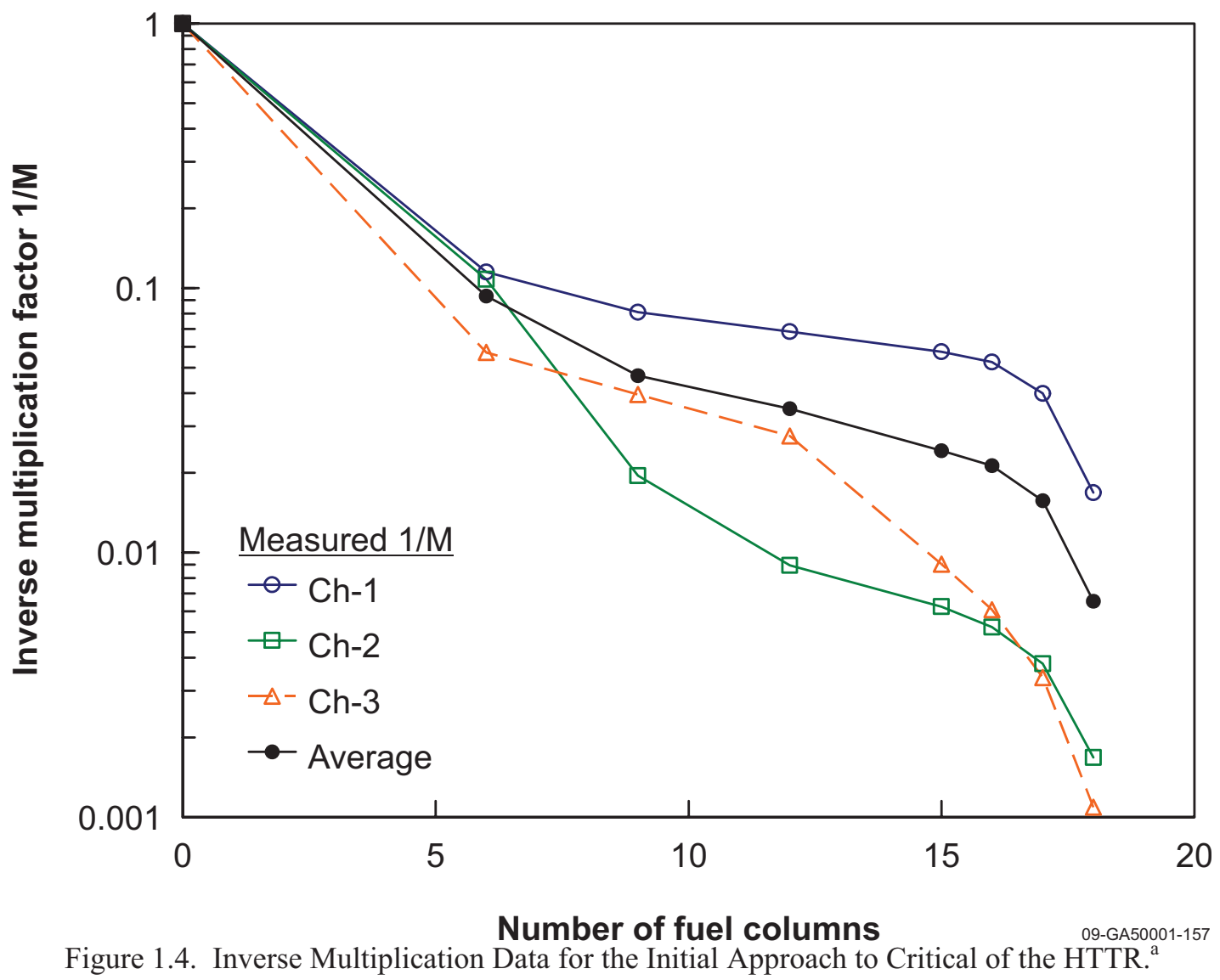

During the first approach to critical (after loading the $19^{\text {th }}$ fuel assembly) the source criticality at very low power was achieved. Then the neutron source was removed and the central control rod moved to compensate for the change in reactivity. The first criticality of the reactor was attained on November 10, 1998. Figure 1.5 shows the result of the reactivity measurements during this approach to critical (Ref. 2, p. 113).

\footnotetext{
a "Present Status of HTGR Research and Development," Japan Atomic Energy Research Institute, Tokaimura, March 2004.
} 
NEA/NSC/DOC(2006)1

Gas Cooled (Thermal) Reactor - GCR

HTTR-GCR-RESR-002

CRIT-REAC-RRATE

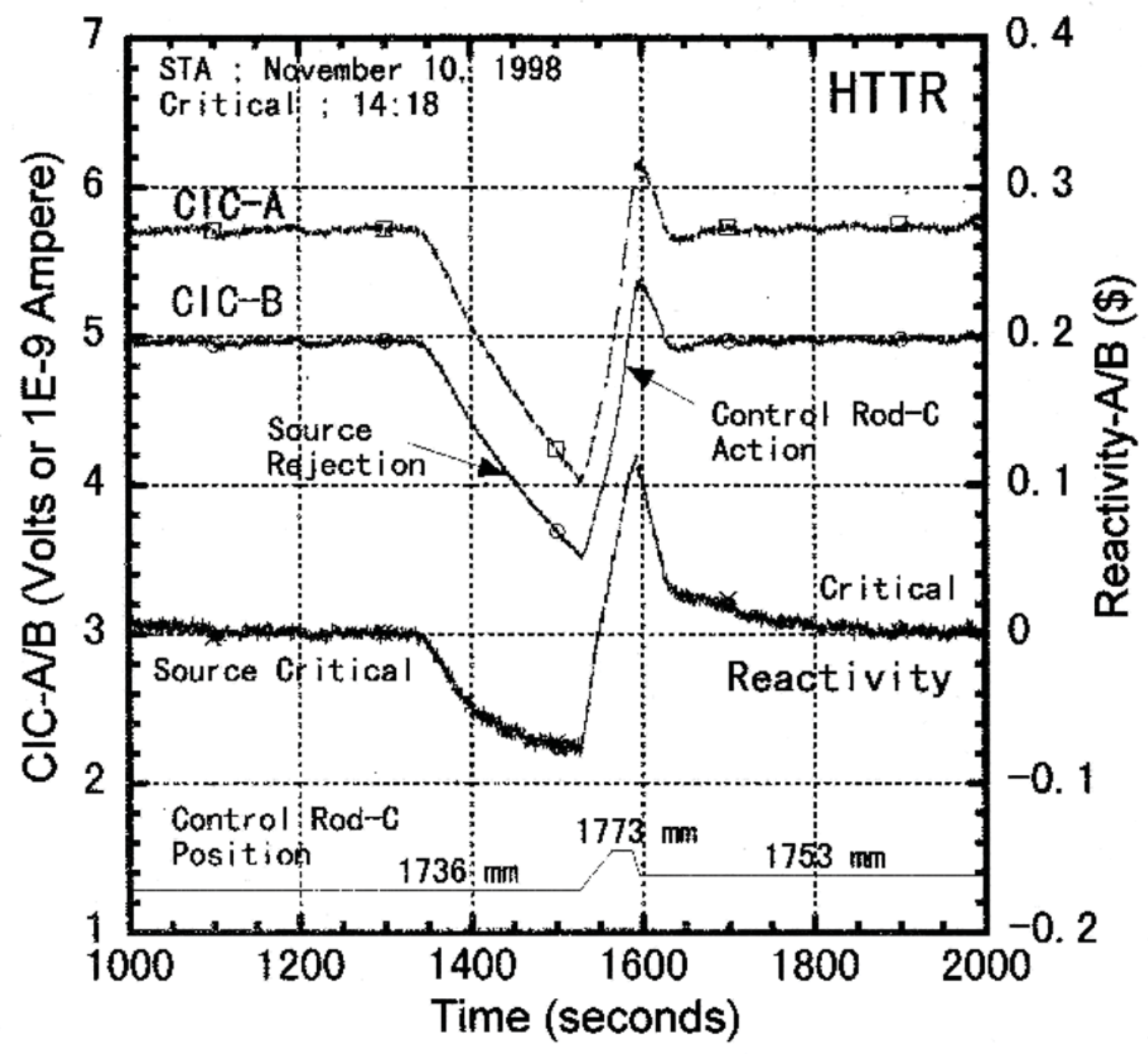

Figure 1.5. Initial Approach to Criticality Reactivity Measurements (Ref 2, p. 114).

The control rod (CR) positions are provided in Table 1.1 for the critical conditions of the annular and fully-loaded cores. The CR pairs were named center (C), ring 1 (R1), ring 2 (R2), and ring 3 (R3) from the center to the outside as shown in Figure 1.2 (also see Section 3.1.2.11 and Figure 3.17). Both R2 and R3 CRs are in the replaceable reflector region surrounding the core, where the six CR pairs on the sides of the hexagonal loading pattern are R2 CRs and the three remaining CR pairs at corners of the hex are R3 CRs. The C-CR was inserted into the 19-column core to adjust initial criticality while the other CRs were fully withdrawn (Ref. 1, p. 317).

All control rod insertion levels are adjusted on the same level except for the three pairs of control rods in the most outer region in the side reflectors. These three pairs of CRs are usually fully withdrawn (Ref. 2, p. 40).

There were two CR patterns used for the 24-fuel-column core. The first was the flat standard (FS) pattern, where the $\mathrm{C}, \mathrm{R} 1$, and R2 CRs were inserted into the core at the same height, and the R3 CRs were fully withdrawn. The second pattern was the F23 pattern, where the R2 and R3 CRs in the side reflector were inserted into the core at the same height and the C and R1 CRs were fully withdrawn. The F23 pattern was used to simulate the control of future HTGRs, where the reflector CRs would mainly be 
NEA/NSC/DOC(2006)1

\section{Gas Cooled (Thermal) Reactor - GCR \\ HTTR-GCR-RESR-002 \\ CRIT-REAC-RRATE}

used for reactivity control. The effective multiplication factors for the measured CR positions at critical conditions were evaluated for different annular cores and temperatures. The results in Table 1.1 are adjusted such that the multiplication factors could be evaluated at $27^{\circ} \mathrm{C}, 300 \mathrm{~K}$ (Ref. 1, pp. 317-318).

A simpler statement for control rod positions of four of the critical cases is shown in Table 1.2, representing the average for rods $\mathrm{C}, \mathrm{R} 1$, and $\mathrm{R} 2$ (with R3 completely withdrawn).

Effective corrections to critical rod position in the core include a sinking effect from the CR driving mechanism of about $-14 \mathrm{~mm}$ and a temperature expansion effect of about $+2 \mathrm{~mm}$ from 25 to $27^{\circ} \mathrm{C}$ (Ref. 2 , p. 46). Reported Japanese data typically had the sinking effect already accounted for in the reported rod positions.

Control rods positions were defined with distance from the bottom of the fifth fuel layer (Ref. 1, p. 317).

Table 1.1. Measured Control Rod Positions for Critical Conditions (Ref. 1, p. 318).

\begin{tabular}{|c|c|c|c|c|c|c|c|c|}
\hline \multirow{2}{*}{ Case } & \multirow{2}{*}{$\begin{array}{c}\text { Fuel } \\
\text { Columns }\end{array}$} & \multirow{2}{*}{$\begin{array}{c}\text { Temperature } \\
\left({ }^{\circ} \mathrm{C}\right)\end{array}$} & \multirow{2}{*}{$\mathrm{k}_{\mathrm{m}}^{(\mathrm{a})}$} & \multicolumn{4}{|c|}{ Critical Rod Position $(\mathrm{mm})^{(\mathrm{b})}$} & \multirow{2}{*}{$\operatorname{Remark}^{(\mathrm{c})}$} \\
\hline & & & & $\mathrm{C}$ & $\mathrm{R} 1$ & $\mathrm{R} 2$ & $\mathrm{R} 3$ & \\
\hline 1 & 19 & 23 & 1.00049 & 1739 & 4050 & 3325 & 4050 & $\mathrm{C}$ \\
\hline 2 & 21 & 24 & 1.00037 & 2647 & 2645 & 2646 & 4049 & FS \\
\hline 3 & 24 & 24 & 1.00037 & 2213 & 2215 & 2215 & 4049 & FS \\
\hline 4 & 24 & 24 & 1.00037 & 4051 & 4050 & 1593 & 1592 & F23 \\
\hline 5 & 27 & 24 & 1.00037 & 1901 & 1899 & 1899 & 4050 & FS \\
\hline 6 & 30 & 25 & 1.00025 & 1775 & 1775 & 1775 & 4049 & FS \\
\hline
\end{tabular}

(a) Measured $\mathrm{k}_{\mathrm{m}}$ has been corrected with the measured temperature coefficient (TC) for the 30 -fuel-column core $\left[-1.23 \times 10^{-4}\left(\Delta \mathrm{k} / \mathrm{k} /{ }^{\circ} \mathrm{C}\right)\right.$ to $\left.27^{\circ} \mathrm{C}(300 \mathrm{~K})\right]$. It was assumed that use of the TC for the 30 -fuel-column core was practically still useful for the other cores because of the small temperature difference of $<4$ ${ }^{\circ} \mathrm{C}$.

(b) Nominally fully withdrawn positions of C, R1, and R3 CRs are $4060 \mathrm{~mm}$ and that of the R2 CRs is $3335 \mathrm{~mm}$.

(c) $\mathrm{C}=$ criticality obtained using central control rod only. FS = flat standard pattern where C, R1, and R2 CRs were inserted into the core at the same levels while R3 CRs were fully withdrawn. F23 = only R2 and R3 CRs were used for control while $\mathrm{C}$ and $\mathrm{R} 1 \mathrm{CRs}$ were fully withdrawn.

Table 1.2. Average Control Rod Positions for Critical Conditions (Ref. 2, p. 40).

\begin{tabular}{|c|c|c|c|c|c||}
\hline Fuel Column & $\begin{array}{c}21 \\
\text { (Case 2) }\end{array}$ & $\begin{array}{c}24 \\
(\text { Case 3) }\end{array}$ & $\begin{array}{c}27 \\
\text { (Case 5) }\end{array}$ & $\begin{array}{c}30 \\
\text { (Case 6) }\end{array}$ \\
\hline \hline Rod Position (mm) & $2646 \pm 5$ & $2215 \pm 5$ & $1899 \pm 5$ & $1775 \quad \pm \quad 5$ \\
\hline \hline
\end{tabular}




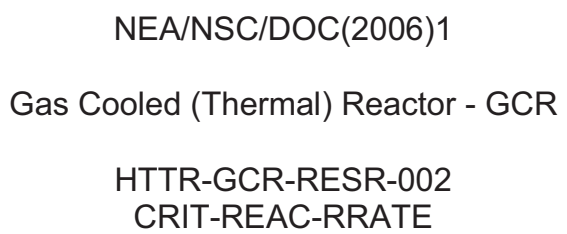

\subsubsection{Material Data}

The material data publicly available for this benchmark can be found compiled in Section 1.1.3 of HTTR-GCR-RESR-001.

\subsubsection{Temperature Data}

The core is at room temperature (Ref. 2, p. 14). Table 1.1 provides the temperature of the experiment for each of the six critical configurations, ranging between 23 and $25^{\circ} \mathrm{C}$.

\subsubsection{Additional Information Relevant to Critical and Subcritical Measurements}

Additional information is not available.

\subsection{Description of Buckling and Extrapolation Length Measurements}

Buckling and extrapolation length measurements were not made.

\section{$1.3 \quad$ Description of Spectral Characteristics Measurements}

Spectral characteristics measurements were not made.

\section{$1.4 \quad$ Description of Reactivity Effects Measurements}

After the initial criticality the excess reactivity of the core was then obtained by adding all increments of the reactivity from the first criticality to the fully-loaded core (Ref. 2, p. 14).

Further information regarding the measurement of excess reactivity of the annular core configurations is provided in Section 1.4.2.1 of HTTR-GCR-RESR-001.

\subsection{Description of Reactivity Coefficient Measurements}

Reactivity coefficient measurements were not made.

\subsection{Description of Kinetics Measurements}

Kinetics measurements were not made.

\subsection{Description of Reaction-Rate Distribution Measurements}

\subsubsection{Overview of Experiment}

The axial reaction rate profile in the instrumentation columns has been evaluated for this benchmark configuration. 
NEA/NSC/DOC(2006)1

Gas Cooled (Thermal) Reactor - GCR

HTTR-GCR-RESR-002

CRIT-REAC-RRATE

\subsubsection{Geometry of the Experiment Configuration and Measurement Procedure}

The geometry of the core is that of the fully-loaded core configuration in Section 1.1.2 with modifications as stated below.

\subsubsection{Axial Reaction Rate Distribution}

The fission reaction rate of ${ }^{235} \mathrm{U}$ was regarded as a neutron flux distribution because the neutron flux distribution could not be directly measured in the HTTR core. The three micro fission chambers (FCs) placed in the irradiation test columns were used to measure the axial reaction rate. The FCs are $5 \mathrm{~cm}$ long with a diameter of $0.6 \mathrm{~cm}$. A FC was connected to the end of a long aluminum stick such that it could be axially traversed by moving the stick in the aluminum tube well inserted into the holes of the irradiation test columns. The temporary neutron source was withdrawn from the core during measurements. The reaction rates were then measured at several points around the peak of the reaction rate distribution to search for the peak. The reactor power was changed by the movement of the FC because reactivity was added by movement of the attached stick and cable, which caused a change in the reaction rates of the FCs. The reaction rate of the traversing FC was then normalized with the reaction rate of the FC fixed in another irradiation test column. The axial fission reaction rate distributions were measured for the 24- and 30-fuel-column cores. Both FS and F23 patterns were formed in the 24-fuelcolumn core. The experimental error of the neutron flux was $\sim 0.2 \%$, and was evaluated from the number of FC pulse counts. Measured distributions are shown (compared with some of Japan's calculated results) in Figure 1.6 (Ref. 1, pp. 317-318).

The reaction rate for the 30-fuel-column core configuration is evaluated in HTTR-GCR-RESR-001, as it represents measurements performed using the fully-loaded core configuration of the HTTR. 
NEA/NSC/DOC(2006)1

Gas Cooled (Thermal) Reactor - GCR

HTTR-GCR-RESR-002

CRIT-REAC-RRATE

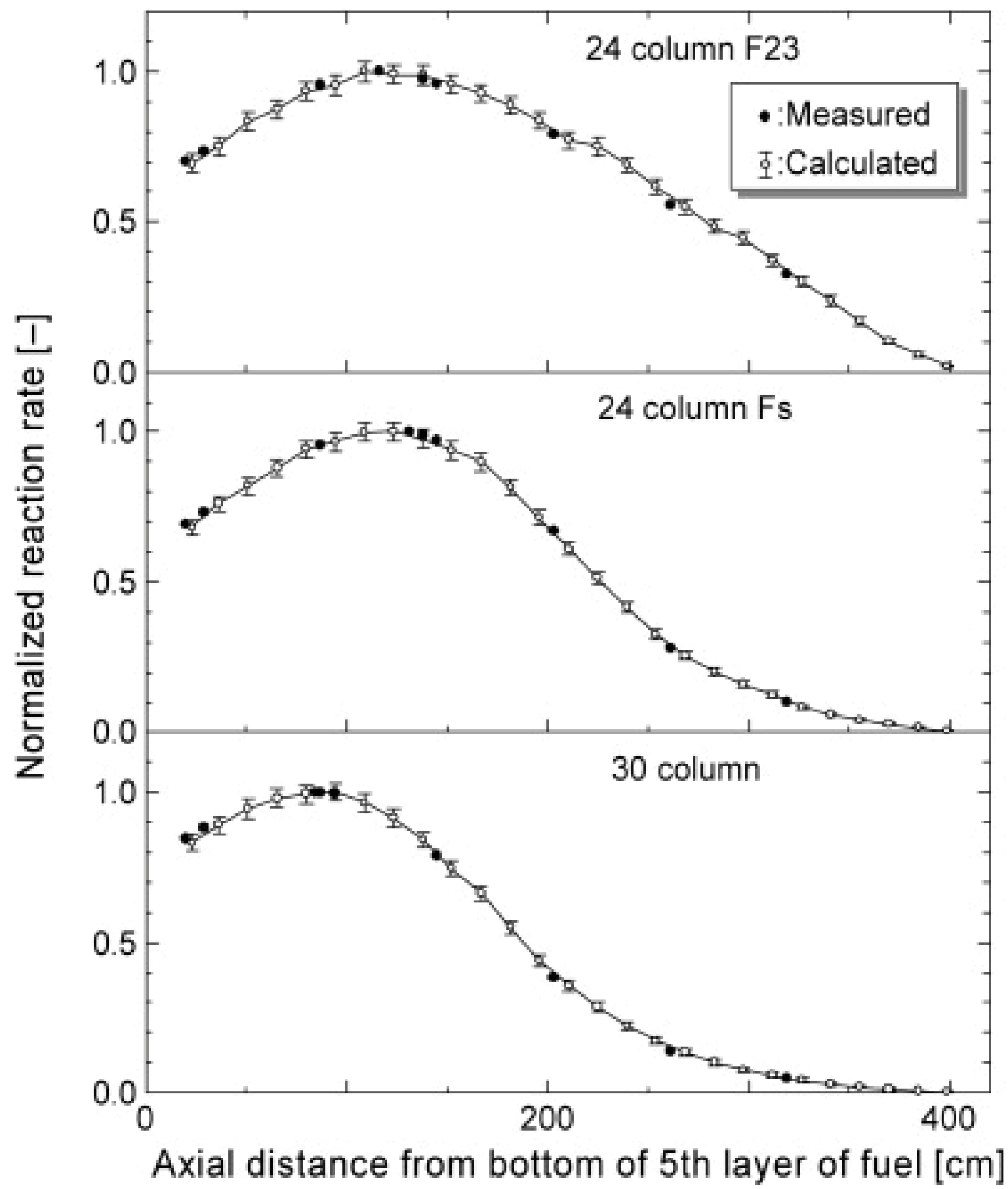

Figure 1.6. Axial Reaction Rate Distribution in Irradiation Column for 24- and 30-Fuel-Column Cores (Ref. 1, p. 318). F23 and FS are defined in the footnote of Table 1.1. The 30-fuel-column core reactionrate measurements are evaluated in HTTR-GCR-RESR-001. 


\section{NEA/NSC/DOC(2006)1 \\ Gas Cooled (Thermal) Reactor - GCR \\ HTTR-GCR-RESR-002 \\ CRIT-REAC-RRATE}

\subsubsection{Material Data}

The materials in the core were those described in the fully-loaded core configuration in Section 1.1.3 with modifications as stated below.

\subsubsection{Temperature Data}

Experiments were essentially performed at room temperature.

\subsubsection{Additional Information Relevant to Reaction-Rate Distribution Measurements}

Additional information is not available.

\subsection{Description of Power Distribution Measurements}

Power distribution measurements were not made.

\section{$1.9 \quad$ Description of Isotopic Measurements}

Isotopic measurements were not made.

\subsection{Description of Other Miscellaneous Types of Measurements}

Other miscellaneous types of measurements were not made. 
NEA/NSC/DOC(2006)1

Gas Cooled (Thermal) Reactor - GCR

HTTR-GCR-RESR-002

CRIT-REAC-RRATE

\subsection{EVALUATION OF EXPERIMENTAL DATA}

The overall uncertainty in the calculated value of $\mathrm{k}_{\text {eff, }}$ which is a function of multiple input parameters, is given by

$$
u_{c}^{2}\left(k_{e f f}\right)=\sum_{i=1}^{N}\left(\Delta k_{i}\right)^{2}+2 \sum_{i=1}^{N-1} \sum_{j=i+1}^{N}\left(\Delta k_{i}\right)\left(\Delta k_{j}\right) r_{i, j}
$$

In Equation 2.1, $\Delta k_{i}$ is the change in $\mathrm{k}_{\mathrm{eff}}$ when parameter $i$ is changed by the standard deviation in the parameter, and $r_{i, j}$ is the correlation coefficient for parameters $i$ and $j$.

Where standard deviations are available, they are used for calculating the effects these uncertainties might have on $\mathrm{k}_{\text {eff, }}$ in terms of $\Delta k_{i}$. Where observed ranges are given, but not standard deviations, the limiting values of the observed ranges are usually applied, and plausible distribution functions are assumed for finding $\Delta k_{i}$. Where only tolerances are given, their limiting values are used, along with plausible distribution functions. Where no guidance is given on the variability of a parameter, engineering judgment is used to select a range of variation that will produce the largest reasonable uncertainty in $\mathrm{k}_{\text {eff. }}$ The bounding values in this range are then applied in the uncertainty analysis. If the overall uncertainty in $\mathrm{k}_{\text {eff }}$ predicted by this approach is small enough that the experiment can be judged an acceptable benchmark, one can be confident that the real experiment is actually even better. All uncertainties are adjusted to values of one standard deviation $(1 \sigma)$. No information is available on correlations among parameters, so all parameters and their uncertainties are assumed to be uncorrelated.

Usually, no information is publicly available about the distribution function of the deviation of a parameter from its nominal value. In most cases, it is reasonably assumed that the most relevant quantity is uniformly distributed. For example, if the change in $\mathrm{k}_{\text {eff }}$ from its nominal value is dependent on the change in the volume of a spatial region, then it is assumed that the deviation of the volume of that region from its nominal value is uniformly distributed.

The uncertainty analyses were performed in accordance with guidance provided in the ICSBEP Handbook.

It should be noted that assuming a uniform distribution of a parameter between its limits leads to overprediction of the effect on $\mathrm{k}_{\mathrm{eff}}$.

These observations are used repeatedly in the following analysis.

The following sections discuss the calculation of the effects of uncertainties in the parameters listed in tables in each section. The values of the tabulated parameters are computed in the benchmark critical configuration and in the configuration with each parameter assigned its maximum variation (or its standard deviation when available), one parameter at a time. The bases for the choices of the parameter values are discussed.

In all cases where tolerances or observed variations apply to large numbers of objects, such as TRISO fuel particles, both deterministic (or systematic) uncertainties (applying to all the objects equally) and random uncertainties (different from one object to the next) will occur. For the fuel particles and their subregions especially, the random uncertainties are extremely small (i.e., the tolerance limit for the random uncertainty divided by the square root of the number of fuel particles in the core). In all cases, division by such large numbers would make the random component of the uncertainty negligible.

\footnotetext{
a International Handbook of Evaluated Criticality Safety Benchmark Experiments, NEA/NSC/DOC(95)03/I-VIII, OECD-NEA, "ICSBEP Guide to the Expression of Uncertainties," Revision 1, p. 29, September 30, 2004.
} 
NEA/NSC/DOC(2006)1

\section{Gas Cooled (Thermal) Reactor - GCR \\ HTTR-GCR-RESR-002 \\ CRIT-REAC-RRATE}

Positional dependence of objects within the assembly also influences the effective proportional effect on the resultant uncertainty and bias calculations. However, since no information is available about how the uncertainties are divided between the systematic and random components, it is assumed throughout that the uncertainties are $25 \%$ systematic and $75 \%$ random for all uncertainties that exhibit a random component.

This assumption provides a basic prediction of the effect on $\mathrm{k}_{\text {eff }}$ until additional information regarding systematic uncertainties can be better evaluated. The $25 \%$ systematic uncertainty is bound by the fact that most systematic uncertainties would be below $50 \%$ of the total uncertainty and above the historic approach of ignoring the unknown systematic components (i.e., treat it with a $0 \%$ probability). In actuality, careful experimenters may have an unknown systematic uncertainty that is approximately 10$15 \%$ of their total reported uncertainty. Evaluated uncertainties are listed as calculated, such that the readers may themselves adjust results according to some desired systematic-to-random uncertainty ratio. The summary in Section 2.1.7 does list the systematic and random components of the uncertainty as separate entities based on the assumption that uncertainties with random components have $25 \%$ systematic uncertainty.

It is important to note that most parameters regarding the TRISO particles are normally distributed.

\subsection{Evaluation of Critical and / or Subcritical Configuration Data}

In the preliminary Japanese computational evaluation, the predicted number of fuel columns to achieve criticality was $16 \pm 1$, much less than the experimental result of 19 . It was regarded that the neglect of nitrogen in the porous graphite led to this discrepancy. Two reevaluations were carried out after the initial criticality experiment (Ref. 1, pp. 313-314). The first reevaluation examined the air content in the graphite, simplification of graphite geometry, and impurity concentration in the dummy blocks. The predicted number of fuel columns for initial criticality was changed to $18 \pm 1$. $^{\text {a }}$ The second reevaluation wasn't performed until after the full power tests at $30 \mathrm{MW}$ and $850{ }^{\circ} \mathrm{C}$. Heterogeneity effects and air composition in the graphite was analyzed; the reevaluation also predicted $18 \pm 1$ fuel columns for initial criticality.

Monte Carlo n-Particle (MCNP) version 5.1.40 calculations were utilized to estimate the biases and uncertainties associated with the experimental results for HTTR critical configurations in this evaluation. MCNP is a general-purpose, continuous-energy, generalized-geometry, time-dependent, coupled n-

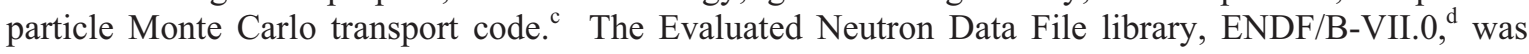
utilized in analysis of the experiment and benchmark model biases and uncertainties.

Elemental data such as molecular weights and isotopic abundances were taken from the $16^{\text {th }}$ edition of

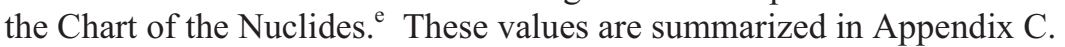

\footnotetext{
${ }^{a}$ Fujimoto, N., Nakano, M., Takeuchi, M., Fujisaki, S., and Yamashita, K., "Start-Up Core Physics Tests of High Temperature Engineering Test Reactor (HTTR), (II): First Criticality by an Annular Form Fuel Loading and Its Criticality Prediction Method,” J. Atomic Energy Society Japan, 42(5), 458-464 (2000).

${ }^{\mathrm{b}}$ Yamashita, K., Fujimoto, N., Takeuchi, M., Fujisaki, S., Nakano, M., Umeda, M., Takeda, T., Mogi, H., and Tanaka, T., "Startup Core Physics Tests of High Temperature Engineering Test Reactor (HTTR), (I): Test Plan, Fuel Loading and Nuclear Characteristics Tests," J. Atomic Energy Society Japan, 42(1), 30-42 (2000).

c X-5 Monte Carlo Team, "MCNP - a General Monte Carlo n-Particle Transport Code, version 5," LA-UR-031987, Los Alamos National Laboratory (2003).

${ }^{\text {d }}$ M. B. Chadwick, et al., "ENDF/B-VII.0: Next Generation Evaluated Nuclear Data Library for Nuclear Science and Technology," Nucl. Data Sheets, 107: 2931-3060 (2006).

${ }^{\mathrm{e}}$ Nuclides and Isotopes: Chart of the Nuclides, $16^{\text {th }}$ edition, (2002).
} 
NEA/NSC/DOC(2006)1

\section{Gas Cooled (Thermal) Reactor - GCR \\ HTTR-GCR-RESR-002 \\ CRIT-REAC-RRATE}

Only the primary components of the HTTR active core and surrounding reflector region were included in the analysis of uncertainties in this evaluation. The uncertainty analysis was performed using a model temperature of approximately $300 \mathrm{~K}$. Five configurations, or cases, representing the HTTR annular cores, created during initial fuel loading, are analyzed in this benchmark. The fully-loaded core configuration is analyzed in a separate report (HTTR-GCR-RESR-001). The difference between the different cases is the number of loaded fuel columns in the core; these are identified in Table 1.1.

For all impurity assessments, only the equivalent natural-boron content is utilized in the evaluation. In the compositions used in the evaluation models, the natural-boron content is adjusted to include only the primary absorber ${ }^{10} \mathrm{~B}$, according to an isotopic abundance of 19.9 at. $\%$.

All MCNP calculations of $\mathrm{k}_{\mathrm{eff}}$ have statistical uncertainties between 0.00011 and 0.00012 , resulting in $\Delta \mathrm{k}$ statistical uncertainties of approximately 0.00016 , assuming no correlation between the individual MCNP results.

Some of the calculated uncertainties are poorly estimated because they are very small and on the order of the statistical uncertainty of the analysis method. However, these uncertainties are insignificant in magnitude compared to the total benchmark uncertainty. Reanalysis of most of these parameters with larger variations would not significantly reduce their uncertainties below the statistical uncertainty of the Monte Carlo calculations.

Uncertainties less than 0.00001 are reported as negligible (neg). When calculated uncertainties in $\Delta \mathrm{k}_{\mathrm{eff}}$ are less than their statistical uncertainties, the statistical uncertainties are used in the calculation of the total uncertainty.

The term "Scaling Factor" denotes the necessary correction to adjust the evaluated uncertainty in $\mathrm{k}_{\text {eff }}$ to a $1 \sigma$ value. Often a larger uncertainty is evaluated such that the calculated $\Delta \mathrm{k}$ value is greater than the statistical uncertainty in the analysis method.

\subsubsection{Experimental Uncertainties}

\subsubsection{Temperature}

Temperature coefficients were not determined for the annular core configurations. The experimenters assumed that the temperature coefficient for the fully-loaded core configuration applies toward all HTTR configurations. Various temperature coefficients of reactivity are reported, ranging from $-1.23 \times 10^{-4}$ $\Delta \mathrm{k} / \mathrm{k}-{ }^{\circ} \mathrm{C}$ (Table 1.17 of HTTR-GCR-RESR-001) to $-14.2 \mathrm{pcm} /{ }^{\circ} \mathrm{C}$ (Ref. 2, p. 113 and 132). An average of $-13.25 \mathrm{pcm} /{ }^{\circ} \mathrm{C}$ with a deviation of $\pm 0.95(1 \sigma)$ was selected to represent the effective change in reactivity with temperature adjustment. The experiments were performed near room temperature, and an uncertainty of $\pm 1^{\circ} \mathrm{C}(1 \sigma)$ was selected to represent the uncertainty in the temperature of the experiment. Results are shown in Table 2.1.

The uncertainty in the temperature is considered all systematic with no random component.

Table 2.1. Effect of Uncertainty in Temperature.

\begin{tabular}{||c|ccc|c|ccc||}
\hline \hline Deviation & $\Delta \mathrm{k}$ & \pm & $\sigma_{\Delta \mathrm{k}}$ & Scaling Factor & $\Delta \mathrm{k}_{\mathrm{eff}}(1 \sigma)$ & \pm & $\sigma_{\Delta \mathrm{keff}}$ \\
\hline \hline $\begin{array}{c}-1{ }^{\circ} \mathrm{C} \\
+1{ }^{\circ} \mathrm{C}\end{array}$ & 0.00013 & \pm & 0.00001 & 1 & 0.00013 & \pm & 0.00001 \\
\hline
\end{tabular}


NEA/NSC/DOC(2006)1

\section{Gas Cooled (Thermal) Reactor - GCR \\ HTTR-GCR-RESR-002 \\ CRIT-REAC-RRATE}

\subsubsection{Control Rod Position}

Control rod positions were varied $\pm 15 \mathrm{~mm}(3 \sigma)$, as shown in Table 1.2, to determine the effective change in $\mathrm{k}_{\text {eff. }}$ The "sinking effect" of $-14 \mathrm{~mm}$ that was described in Section 1.1.2.1 (just prior to Table 1.1) had already been applied to the rod position shown in Tables 1.1 and 1.2. It is unconfirmed whether temperature expansion effect of $\pm 2 \mathrm{~mm}$ (reported at the end of Section 1.1.2.4 in HTTR-GCR-RESR-001) applies uniformly to all control rods, how it was obtained, or that is was even applied to the reported experimental positions. Therefore it is applied with the $\pm 5 \mathrm{~mm}$ in a root mean square approach, the overall uncertainty in the height remains approximately $\pm 5 \mathrm{~mm}$. No additional bias or bias uncertainty was evaluated for the sinking effect of the control rods. Results are shown in Table 2.2 .

The total number of control rods used in the core is 32 (16 pairs). For determining the random component of the uncertainty in Section 2.1.7, the results in Table 2.2 are divided by $\sqrt{16}$.

Table 2.2. Effect of Uncertainty in Control Rod Position.

\begin{tabular}{||c|c|ccc|c|ccc||}
\hline \hline Case & Deviation & $\Delta \mathrm{k}$ & \pm & $\sigma_{\Delta \mathrm{k}}$ & Scaling Factor & $\Delta \mathrm{k}_{\text {eff }}(1 \sigma)$ & \pm & $\sigma_{\Delta \mathrm{keff}}$ \\
\hline \hline 1 & $-15 \mathrm{~mm}(3 \sigma)$ & -0.00036 & \pm & 0.00017 & 3 & -0.00012 & \pm & 0.00006 \\
& $+15 \mathrm{~mm}(3 \sigma)$ & 0.00041 & \pm & 0.00017 & 3 & 0.00014 & \pm & 0.00006 \\
\hline 2 & $-15 \mathrm{~mm}(3 \sigma)$ & -0.00133 & \pm & 0.00017 & 3 & -0.00044 & \pm & 0.00006 \\
& $+15 \mathrm{~mm}(3 \sigma)$ & 0.00142 & \pm & 0.00016 & 3 & 0.00047 & \pm & 0.00005 \\
\hline \multirow{2}{*}{3} & $-15 \mathrm{~mm}(3 \sigma)$ & -0.00175 & \pm & 0.00016 & 3 & -0.00058 & \pm & 0.00005 \\
& $+15 \mathrm{~mm}(3 \sigma)$ & 0.00156 & \pm & 0.00017 & 3 & 0.00052 & \pm 0.00006 \\
\hline \multirow{2}{*}{4} & $-15 \mathrm{~mm}(3 \sigma)$ & -0.00111 & \pm & 0.00017 & 3 & -0.00037 & \pm & 0.00006 \\
& $+15 \mathrm{~mm}(3 \sigma)$ & 0.00076 & \pm & 0.00017 & 3 & 0.00025 & \pm & 0.00006 \\
\hline 5 & $-15 \mathrm{~mm}(3 \sigma)$ & -0.00169 & \pm 0.00016 & 3 & -0.00056 & \pm & 0.00005 \\
& $+15 \mathrm{~mm}(3 \sigma)$ & 0.00187 & \pm & 0.00016 & 3 & 0.00062 & \pm & 0.00005 \\
\hline \hline
\end{tabular}

\subsubsection{Measured Value of $k_{\text {eff }}$}

There is no additional information regarding the accuracy of the $\mathrm{k}_{\mathrm{eff}}$ measurements for the critical core conditions reported in Table 1.1. Typically the uncertainty and its effect are relatively insignificant.

\subsubsection{Geometrical Properties}

\subsubsection{Coated Fuel Particles}

When adjusting the diameters of the TRISO particle coatings, the other diameters are held constant. This in turn would increase or decrease the mass of the remaining layers. Whereas the diameters of the different layers appeared to be documented with more detail (see Table 1.14 and Figure 1.48 of HTTR-GCR-RESR-001), this approach seemed appropriate. The uncertainty in the mass is then evaluated as part of the density uncertainty Section 2.1.3.1.

\section{Kernel Diameter}

Because of the overspecification of the TRISO particles in Table 1.14 (HTTR-GCR-RESR-001) and the correlation of uranium kernel diameter, density, TRISO packing fraction, and mass, the effect of the 
NEA/NSC/DOC(2006)1

\section{Gas Cooled (Thermal) Reactor - GCR \\ HTTR-GCR-RESR-002 \\ CRIT-REAC-RRATE}

uncertainty in the kernel diameter is not included in the total uncertainty. However, an analysis of the uncertainty based upon the fuel mass is performed in Section 2.1.6.

\section{Buffer Diameter}

The buffer thickness was varied $\pm 12 \mu \mathrm{m}(3 \sigma)$ from the nominal value of $60 \mu \mathrm{m}$ (Tables 1.12 and 1.14, and Figure 1.48 of HTTR-GCR-RESR-001) to determine the effective change in $\mathrm{k}_{\text {eff. }}$ All other thicknesses in the TRISO particle were maintained the same. The packing fraction of the TRISO particles in the fuel was not conserved, so as to conserve fuel mass, but remained within $1 \sigma$ of the nominal value of 30 vol. $\%$ ( $\pm 3 \%$ in Table 1.14 of HTTR-GCR-RESR-001). Results are shown in Table 2.3 .

The total number of TRISO particles used in the fully-loaded core is approximately 868,140,000. For determining the random component of the uncertainty, the results in Table 2.3 would be divided by $\sqrt{\mathrm{N}}$, where $\mathrm{N}$ for each case is shown in Table 2.3.

Table 2.3. Effect of Uncertainty in Buffer Diameter.

\begin{tabular}{|c|c|c|c|c|c|c|c|c|c|}
\hline Case & Deviation & $\Delta \mathrm{k}$ & \pm & $\sigma_{\Delta \mathrm{k}}$ & $\begin{array}{l}\text { Scaling } \\
\text { Factor }\end{array}$ & $\Delta \mathrm{k}_{\text {eff }}(1 \sigma)$ & \pm & $\sigma_{\Delta \mathrm{keff}}$ & $\mathrm{N}$ \\
\hline \multirow{2}{*}{1} & & & \pm & & 3 & & \pm & 05 & \multirow{2}{*}{$5.38 \times 10^{8}$} \\
\hline & $+12 \mu$ & 0.00000 & \pm & 0.00017 & 3 & 0.00000 & \pm & 0.00006 & \\
\hline \multirow{2}{*}{2} & -12 & -0.00005 & \pm & 0.00016 & 3 & -0.00002 & \pm & 006 & \multirow{2}{*}{$5.98 \times 10^{8}$} \\
\hline & $+12 \mu$ & -0.00016 & \pm & 0.00017 & 3 & -0.00005 & \pm & 0.00006 & \\
\hline \multirow{2}{*}{3} & -1 & 19 & \pm & 0. & 3 & 0006 & \pm & 0.00005 & \multirow{2}{*}{$6.88 \times 10^{8}$} \\
\hline & $+12 \mu \mathrm{m}(3 \sigma)$ & -0.00015 & \pm & 0.00017 & 3 & -0.00005 & \pm & 0.00006 & \\
\hline \multirow{2}{*}{4} & $-12 \mu \mathrm{m}(3 \sigma)$ & 0.00002 & \pm & 0.00017 & 3 & 0.00001 & \pm & 0.00006 & \multirow{2}{*}{$6.88 \times 10^{8}$} \\
\hline & $+12 \mu \mathrm{m}(3 \sigma)$ & -0.00043 & \pm & 0.00017 & 3 & -0.00014 & \pm & 006 & \\
\hline & $-12 \mu \mathrm{m}(3 \sigma)$ & 0.00028 & \pm & 0.00016 & 3 & 0.00009 & \pm & 0.00005 & \multirow{2}{*}{$7.78 \times 10^{8}$} \\
\hline & $+12 \mu \mathrm{m}(3 \sigma)$ & -0.00022 & \pm & 0.00016 & 3 & -0.00007 & \pm & 0.00005 & \\
\hline
\end{tabular}

\section{IPyC Diameter}

The IPyC thickness was varied $\pm 6 \mu \mathrm{m}(3 \sigma)$ from the nominal value of $30 \mu \mathrm{m}$ (Tables 1.12 and 1.14, and Figure 1.48 of HTTR-GCR-RESR-001) to determine the effective uncertainty in $\mathrm{k}_{\text {eff. }}$ All other thicknesses in the TRISO particle were maintained the same. The packing fraction of the TRISO particles in the fuel was not conserved but remained within $1 \sigma$ of the nominal value of $30 \mathrm{vol} . \%( \pm 3 \%$ in Table 1.14 of HTTR-GCR-RESR-001). Results are shown in Table 2.4.

The total number of TRISO particles used in the fully-loaded core is approximately $868,140,000$. For determining the random component of the uncertainty, the results in Table 2.4 would be divided by $\sqrt{ } \mathrm{N}$, where $\mathrm{N}$ for each case is shown in Table 2.4. 
NEA/NSC/DOC(2006)1

Gas Cooled (Thermal) Reactor - GCR

HTTR-GCR-RESR-002

CRIT-REAC-RRATE

Table 2.4. Effect of Uncertainty in IPyC Diameter.

\begin{tabular}{|c|c|c|c|c|c|c|c|c|c|}
\hline Case & Deviation & $\Delta \mathrm{k}$ & \pm & $\sigma_{\Delta \mathrm{k}}$ & $\begin{array}{l}\text { Scaling } \\
\text { Factor }\end{array}$ & $\Delta \mathrm{k}_{\text {eff }}(1 \sigma)$ & \pm & $\sigma_{\Delta \mathrm{keff}}$ & $\mathrm{N}$ \\
\hline \multirow[b]{2}{*}{$I$} & $-6 \mu \mathrm{m}(3 \sigma)$ & 0.00003 & & 0.00017 & 3 & 0.00001 & \pm & 0.00006 & \multirow{2}{*}{$5.38 \times 10^{8}$} \\
\hline & $+6 \mu$ & 0.00014 & & 0.00016 & 3 & 0.00005 & \pm & 0.00005 & \\
\hline \multirow{2}{*}{2} & $-6 \mu \mathrm{m}(3 \sigma)$ & -0.00002 & \pm & 0.00016 & 3 & -0.00001 & \pm & 0.00005 & \multirow{2}{*}{$5.98 \times 10^{8}$} \\
\hline & $+6 !$ & -0.00002 & & 0.00017 & 3 & -0.00001 & \pm & 0.00006 & \\
\hline \multirow{2}{*}{3} & $-6 \mu \mathrm{m}(3 \sigma)$ & -0.00017 & \pm & 0.00017 & 3 & -0.00006 & \pm & 0.00006 & \multirow{2}{*}{$6.88 \times 10^{8}$} \\
\hline & $+6 \mu \mathrm{m}(3 \sigma)$ & 0.00007 & & 0.00017 & 3 & 0.00002 & \pm & 00006 & \\
\hline \multirow{2}{*}{4} & $-6 \mu \mathrm{m}(3 \sigma)$ & -0.00008 & $\perp$ & 0.00017 & 3 & -0.00003 & \pm & 0.00006 & \multirow{2}{*}{$6.88 \times 10^{8}$} \\
\hline & $+6 \mu \mathrm{m}(3 \sigma)$ & 0.00003 & & 0.00017 & 3 & 0.00001 & \pm & 0.00006 & \\
\hline & $-6 \mu \mathrm{m}(3 \sigma)$ & 0.00010 & & 0.00016 & 3 & 0.00003 & \pm & 0.00005 & \multirow{2}{*}{$7.78 \times 10^{8}$} \\
\hline & $+6 \mu \mathrm{m}(3 \sigma)$ & 0.00004 & \pm & 0.00016 & 3 & 0.00001 & \pm & 0.00005 & \\
\hline
\end{tabular}

\section{$\underline{\text { SiC Diameter }}$}

The $\mathrm{SiC}$ thickness was varied $\pm 12 \mu \mathrm{m}(3 \sigma)$ from the nominal value of $30 \mu \mathrm{m}$ (Tables 1.12 and 1.14, and Figure 1.48 of HTTR-GCR-RESR-001) to determine the effective uncertainty in $\mathrm{k}_{\text {eff. }}$ Although there is a negative component to the deviation in the thickness (Figure 1.48 of HTTR-GCR-RESR-001), TRISO particles with $\mathrm{SiC}$ thicknesses less than $25 \mu \mathrm{m}$ are not acceptable for use as a final product. The thickness of $25 \mu \mathrm{m}$ for the thickness appears to be a manufacturing limit while the thickness of $30 \mu \mathrm{m}$ is more likely to be the actual thickness of the SiC layer. All other thicknesses in the TRISO particle were maintained the same. The packing fraction of the TRISO particles in the fuel was not conserved but remained within $1 \sigma$ of the nominal value of 30 vol. $\%$ ( $\pm 3 \%$ in Table 1.14 of HTTR-GCR-RESR-001). Results are shown in Table 2.5.

The total number of TRISO particles used in the fully-loaded core is approximately 868,140,000. For determining the random component of the uncertainty, the results in Table 2.5 would be divided by $\sqrt{\mathrm{N}}$, where $\mathrm{N}$ for each case is shown in Table 2.5. 
NEA/NSC/DOC(2006)1

Gas Cooled (Thermal) Reactor - GCR

HTTR-GCR-RESR-002

CRIT-REAC-RRATE

Table 2.5. Effect of Uncertainty in SiC Diameter.

\begin{tabular}{|c|c|c|c|c|c|c|c|c|c|}
\hline Case & Deviation & $\Delta \mathrm{k}$ & \pm & $\sigma_{\Delta \mathrm{k}}$ & $\begin{array}{c}\text { Scaling } \\
\text { Factor }\end{array}$ & $\Delta \mathrm{k}_{\mathrm{eff}}(1 \sigma)$ & \pm & $\sigma_{\Delta \mathrm{keff}}$ & $\mathrm{N}$ \\
\hline \multirow{2}{*}{1} & & 19 & \pm & 0.00017 & 3 & 0.00006 & \pm & 0.00006 & \multirow{2}{*}{$5.38 \times 10^{8}$} \\
\hline & +12 & -0.00062 & \pm & 0.00017 & 3 & -0.00021 & \pm & 06 & \\
\hline \multirow{2}{*}{2} & & & \pm & 0.00017 & 3 & & \pm & & \multirow{2}{*}{$5.98 \times 10^{8}$} \\
\hline & +12 & -0.0 & \pm & 16 & 3 & -( & \pm & 05 & \\
\hline \multirow{2}{*}{3} & $-12 \mu \mathrm{m}(3 \sigma)$ & 0.00062 & \pm & 0.00016 & 3 & 0.00021 & \pm & 0.00005 & \multirow{2}{*}{$6.88 \times 10^{8}$} \\
\hline & $+12 \mu$ & -0.00057 & \pm & 0.0 & 3 & -0.00019 & \pm & 06 & \\
\hline \multirow{2}{*}{4} & $-12 \mu \mathrm{m}(3 \sigma)$ & 0.00025 & \pm & 0.00016 & 3 & 0.00008 & \pm & 0.00005 & \multirow{2}{*}{$6.88 \times 10^{8}$} \\
\hline & $+12 \mu \mathrm{m}(3 \sigma)$ & -0.00080 & 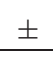 & 0.00017 & 3 & -0.00027 & \pm & 0.00006 & \\
\hline \multirow[t]{2}{*}{5} & $-12 \mu \mathrm{m}(3 \sigma)$ & 0.00068 & \pm & 0.00016 & 3 & 0.00023 & \pm & 0.00005 & \multirow{2}{*}{$7.78 \times 10^{8}$} \\
\hline & $+12 \mu \mathrm{m}(3 \sigma)$ & -0.00074 & \pm & 0.00016 & 3 & -0.00025 & \pm & 0.00005 & \\
\hline
\end{tabular}

\section{OPyC Diameter}

The OPyC thickness was varied $\pm 6 \mu \mathrm{m}(3 \sigma)$ from the nominal value of $45 \mu \mathrm{m}$ (Tables 1.12 and 1.14, and Figure 1.48 of HTTR-GCR-RESR-001) to determine the effective uncertainty in $k_{\text {eff. All other }}$ thicknesses in the TRISO particle were maintained the same. The packing fraction of the TRISO particles in the fuel was not conserved but remained within $1 \sigma$ of the nominal value of $30 \mathrm{vol} . \%( \pm 3 \%$ in Table 1.14 of HTTR-GCR-RESR-001). Results are shown in Table 2.6.

The total number of TRISO particles used in the fully-loaded core is approximately $868,140,000$. For determining the random component of the uncertainty, the results in Table 2.6 would be divided by $\sqrt{\mathrm{N}}$, where $\mathrm{N}$ for each case is shown in Table 2.6.

Table 2.6. Effect of Uncertainty in OPyC Diameter.

\begin{tabular}{|c|c|c|c|c|c|c|c|c|c|}
\hline Case & Deviation & $\Delta \mathrm{k}$ & \pm & $\sigma_{\Delta \mathrm{k}}$ & $\begin{array}{l}\text { Scaling } \\
\text { Factor }\end{array}$ & $\Delta \mathrm{k}_{\mathrm{eff}}(1 \sigma)$ & \pm & $\sigma_{\Delta \mathrm{keff}}$ & $\mathrm{N}$ \\
\hline \multirow{2}{*}{ l } & & -0.00002 & \pm & 0.00016 & 3 & 001 & \pm & 0.00005 & \multirow{2}{*}{$5.38 \times 10^{8}$} \\
\hline & $+6 \mu \mathrm{m}(3 \sigma)$ & 0.00000 & \pm & 0.00017 & 3 & 0.00000 & \pm & 0.00006 & \\
\hline \multirow{2}{*}{2} & $-6 \mu \mathrm{m}(3 \sigma)$ & 0.00019 & \pm & 0.00016 & 3 & 0.00006 & \pm & 0.00005 & \multirow{2}{*}{$5.98 \times 10^{8}$} \\
\hline & $+6 \mu \mathrm{m}(3 \sigma)$ & 0.00032 & \pm & 0.00017 & 3 & 011 & \pm & 0.00006 & \\
\hline \multirow{2}{*}{3} & $-6 \mu \mathrm{m}(3 \sigma)$ & -0.00018 & \pm & 0.00017 & 3 & -0.00006 & \pm & 0.00006 & \multirow{2}{*}{$6.88 \times 10^{8}$} \\
\hline & $+6 \mu \mathrm{m}(3 \sigma)$ & 0.00011 & \pm & 0.00017 & 3 & 0.00004 & \pm & 0.00006 & \\
\hline \multirow{2}{*}{4} & $-6 \mu \mathrm{m}(3 \sigma)$ & -0.00014 & \pm & 0.00017 & 3 & -0.00005 & \pm & 0.00006 & \multirow{2}{*}{$6.88 \times 10^{8}$} \\
\hline & $+6 \mu \mathrm{n}$ & -0.00031 & \pm & 0.00017 & 3 & -0.00010 & \pm & 0.00006 & \\
\hline \multirow{2}{*}{5} & $-6 \mu \mathrm{m}(3 \sigma)$ & -0.00003 & \pm & 0.00016 & 3 & -0.00001 & \pm & 0.00005 & \multirow{2}{*}{$7.78 \times 10^{8}$} \\
\hline & $+6 \mu \mathrm{m}(3 \sigma)$ & -0.00002 & \pm & 0.00016 & 3 & -0.00001 & \pm & 0.00005 & \\
\hline
\end{tabular}


NEA/NSC/DOC(2006)1

\section{Gas Cooled (Thermal) Reactor - GCR \\ HTTR-GCR-RESR-002 \\ CRIT-REAC-RRATE}

\section{Overcoat Diameter}

Because insufficient data is available for the final composition and density of the graphite overcoat, this layer is being treated with equal properties to that of the surrounding compact graphite matrix (Table 1.14 of HTTR-GCR-RESR-001). Therefore modification of the overcoat diameter would not generate an effective uncertainty in $\mathrm{k}_{\text {eff }}$ beyond statistical uncertainty.

\subsubsection{Prismatic Fuel Compact}

\section{Dimensions}

The inner and outer diameters (ID and OD) of a fuel compact were each individually varied $\pm 0.3 \mathrm{~mm}$ and the height $(\mathrm{H})$ was varied $\pm 0.5 \mathrm{~mm}$ to determine the effective uncertainty in $\mathrm{k}_{\text {eff. }}$. For the effective change in height, the fuel stack height was adjusted by $\pm 7.0 \mathrm{~mm}$ and the effect from a height change in a single fuel compact was then determined by dividing by 14 for the number of compacts in a fuel rod. The nominal values for the inner diameter, outer diameter, and height of the fuel compacts are 10.0,26.0, and $39.0 \mathrm{~mm}$, respectively (Table 1.14 of HTTR-GCR-RESR-001). Results are shown in Tables 2.7 through 2.9. In essence, changing the dimensions of the fuel compact without changing the number of TRISO particles would slightly adjust the packing fraction, but within the uncertainty limits.

Later information was obtained regarding manufacturing tolerances for the fuel compacts. The $\pm 0.1 \mathrm{~mm}$ of the ID and OD represents a bounding limit (with assumed uniform probability) and the effective stack height has a bounding limit (with assumed uniform probability) of $\pm 1.0 \mathrm{~mm}$. ${ }^{\mathrm{a}}$ The appropriate corrections to the scaling factors have been incorporated into the uncertainty analysis of these parameters.

The total number of fuel compacts used in the fully-loaded core is 66,780 . For determining the random component of the uncertainty, the results in Tables 2.7 through 2.9 would be divided by $\sqrt{ } \mathrm{N}$, where $\mathrm{N}$ for each case is shown in Tables 2.7 through 2.9 .

Table 2.7. Effect of Uncertainty in Compact Dimensions (Inner Diameter).

\begin{tabular}{|c|c|c|c|c|c|c|c|c|c|}
\hline Case & Deviation & $\Delta \mathrm{k}$ & \pm & $\sigma_{\Delta \mathrm{k}}$ & $\begin{array}{l}\text { Scaling } \\
\text { Factor }\end{array}$ & $\Delta \mathrm{k}_{\mathrm{eff}}(1 \sigma)$ & \pm & $\sigma_{\Delta \mathrm{keff}}$ & $\mathrm{N}$ \\
\hline \multirow{2}{*}{1} & $-0.3 \mathrm{~mm}(3 \times$ limit $)$ & 0.00016 & \pm & 0.00016 & $3 \sqrt{3}$ & 0.00003 & \pm & 0.00003 & \multirow{2}{*}{41,370} \\
\hline & $+0.3 \mathrm{~mm}(3 \times$ limit $)$ & -0.00003 & \pm & 0.00017 & $3 \sqrt{3}$ & -0.00001 & \pm & 0.00003 & \\
\hline \multirow{2}{*}{2} & $-0.3 \mathrm{~mm}(3 \times$ limit $)$ & 0.00014 & \pm & 0.00016 & $3 \sqrt{3}$ & 0.00003 & \pm & 0.00003 & \multirow{2}{*}{45,990} \\
\hline & $+0.3 \mathrm{~mm}(3 \times$ limit $)$ & -0.00020 & \pm & 0.00016 & $3 \sqrt{3}$ & -0.00004 & \pm & 0.00003 & \\
\hline \multirow{2}{*}{3} & $-0.3 \mathrm{~mm}(3 \times$ limit $)$ & 0.00014 & \pm & 0.00017 & $3 \sqrt{3}$ & 0.00003 & \pm & 0.00003 & \multirow{2}{*}{52,920} \\
\hline & $+0.3 \mathrm{~mm}(3 \times$ limit $)$ & -0.00008 & \pm & 0.00017 & $3 \sqrt{3}$ & -0.00002 & \pm & 0.00003 & \\
\hline \multirow{2}{*}{4} & $-0.3 \mathrm{~mm}(3 \times$ limit $)$ & -0.00004 & \pm & 0.00017 & $3 \sqrt{3}$ & -0.00001 & \pm & 0.00003 & \multirow{2}{*}{52,920} \\
\hline & $+0.3 \mathrm{~mm}(3 \times$ limit $)$ & -0.00037 & \pm & 0.00017 & $3 \sqrt{3}$ & -0.00007 & \pm & 0.00003 & \\
\hline \multirow{2}{*}{5} & $-0.3 \mathrm{~mm}(3 \times$ limit $)$ & 0.00012 & \pm & 0.00016 & $3 \sqrt{3}$ & 0.00002 & \pm & 0.00003 & \multirow{2}{*}{59,850} \\
\hline & $+0.3 \mathrm{~mm}(3 \times$ limit $)$ & -0.00023 & \pm & 0.00016 & $3 \sqrt{3}$ & -0.00004 & \pm & 0.00003 & \\
\hline
\end{tabular}

\footnotetext{
${ }^{a}$ S. Maruyama, K. Yamashita, N. Fujimoto, I. Murata, R. Shindo, and Y. Sudo, "Determination of Hot Spot Factors for Calculation of the Maximum Fuel Temperatures in the Core Thermal and Hydraulic Design of HTTR," JAERIM 88-250, JAEA (November 18, 1988). [in Japanese].
} 
NEA/NSC/DOC(2006)1

\section{Gas Cooled (Thermal) Reactor - GCR \\ HTTR-GCR-RESR-002 \\ CRIT-REAC-RRATE}

Table 2.8. Effect of Uncertainty in Compact Dimensions (Outer Diameter).

\begin{tabular}{|c|c|c|c|c|c|c|c|c|c|}
\hline Case & Deviation & $\Delta \mathrm{k}$ & \pm & $\sigma_{\Delta \mathrm{k}}$ & $\begin{array}{c}\text { Scaling } \\
\text { Factor } \\
\end{array}$ & $\Delta \mathrm{k}_{\mathrm{eff}}(1 \sigma)$ & \pm & $\sigma_{\Delta \mathrm{keff}}$ & $\mathrm{N}$ \\
\hline \multirow{2}{*}{1} & $-0.3 \mathrm{~mm}(3 \times$ limit $)$ & -0.00048 & \pm & 0.00017 & $3 \sqrt{3}$ & -0.00009 & \pm & 0.00003 & \multirow{2}{*}{41,370} \\
\hline & $+0.3 \mathrm{~mm}(3 \times$ limit $)$ & 0.00060 & \pm & 0.00016 & $3 \sqrt{3}$ & 0.00012 & \pm & 0.00003 & \\
\hline \multirow{2}{*}{2} & $-0.3 \mathrm{~mm}(3 \times$ limit $)$ & -0.00050 & \pm & 0.00016 & $3 \sqrt{3}$ & -0.00010 & \pm & 0.00003 & \multirow{2}{*}{45,990} \\
\hline & $+0.3 \mathrm{~mm}(3 \times$ limit $)$ & 0.00049 & \pm & 0.00016 & $3 \sqrt{3}$ & 0.00009 & \pm & 0.00003 & \\
\hline \multirow{2}{*}{3} & $-0.3 \mathrm{~mm}(3 \times$ limit $)$ & -0.00087 & \pm & 0.00016 & $3 \sqrt{3}$ & -0.00017 & \pm & 0.00003 & \multirow{2}{*}{52,920} \\
\hline & $+0.3 \mathrm{~mm}(3 \times$ limit $)$ & 0.00068 & \pm & 0.00016 & $3 \sqrt{3}$ & 0.00013 & \pm & 0.00003 & \\
\hline \multirow{2}{*}{4} & $-0.3 \mathrm{~mm}(3 \times$ limit $)$ & -0.00081 & \pm & 0.00017 & $3 \sqrt{3}$ & -0.00016 & \pm & 0.00003 & \multirow{2}{*}{52,920} \\
\hline & $+0.3 \mathrm{~mm}(3 \times$ limit $)$ & 0.00057 & \pm & 0.00017 & $3 \sqrt{3}$ & 0.00011 & \pm & 0.00003 & \\
\hline \multirow{2}{*}{5} & $-0.3 \mathrm{~mm}(3 \times$ limit $)$ & -0.00066 & \pm & 0.00016 & $3 \sqrt{3}$ & -0.00013 & \pm & 0.00003 & \multirow{2}{*}{59,850} \\
\hline & $+0.3 \mathrm{~mm}(3 \times$ limit $)$ & 0.00059 & \pm & 0.00016 & $3 \sqrt{3}$ & 0.00011 & \pm & 0.00003 & \\
\hline
\end{tabular}

Table 2.9. Effect of Uncertainty in Compact Dimensions (Height).

\begin{tabular}{|c|c|c|c|c|c|c|c|c|c|}
\hline Case & Deviation & $\Delta \mathrm{k}$ & \pm & $\sigma_{\Delta \mathrm{k}}$ & $\begin{array}{c}\text { Scaling } \\
\text { Factor }\end{array}$ & $\Delta \mathrm{k}_{\mathrm{eff}}(1 \sigma)$ & \pm & $\sigma_{\Delta \mathrm{keff}}$ & $\mathrm{N}$ \\
\hline \multirow{2}{*}{1} & $-7.0 \mathrm{~mm}(7 \times$ limit $)$ & -0.00262 & \pm & 0.00016 & $7 \sqrt{3}$ & -0.00022 & \pm & 0.00001 & \multirow{2}{*}{41,370} \\
\hline & $+7.0 \mathrm{~mm}(7 \times$ limit $)$ & 0.00246 & \pm & 0.00017 & $7 \sqrt{3}$ & 0.00020 & \pm & 0.00001 & \\
\hline \multirow{2}{*}{2} & $-7.0 \mathrm{~mm}(7 \times$ limit $)$ & -0.00236 & \pm & 0.00017 & $7 \sqrt{3}$ & -0.00019 & \pm & 0.00001 & \multirow{2}{*}{45,990} \\
\hline & $+7.0 \mathrm{~mm}(7 \times$ limit $)$ & 0.00216 & \pm & 0.00017 & $7 \sqrt{3}$ & 0.00018 & \pm & 0.00001 & \\
\hline \multirow{2}{*}{3} & $-7.0 \mathrm{~mm}(7 \times$ limit $)$ & -0.00260 & \pm & 0.00017 & $7 \sqrt{3}$ & -0.00021 & \pm & 0.00001 & \multirow{2}{*}{52,920} \\
\hline & $+7.0 \mathrm{~mm}(7 \times$ limit $)$ & 0.00191 & \pm & 0.00017 & $7 \sqrt{3}$ & 0.00016 & \pm & 0.00001 & \\
\hline \multirow{2}{*}{4} & $-7.0 \mathrm{~mm}(7 \times$ limit $)$ & -0.00282 & \pm & 0.00017 & $7 \sqrt{3}$ & -0.00023 & \pm & 0.00001 & \multirow{2}{*}{52,920} \\
\hline & $+7.0 \mathrm{~mm}(7 \times$ limit $)$ & 0.00201 & \pm & 0.00017 & $7 \sqrt{3}$ & 0.00017 & \pm & 0.00001 & \\
\hline \multirow{2}{*}{5} & $-7.0 \mathrm{~mm}(7 \times$ limit $)$ & -0.00268 & \pm & 0.00016 & $7 \sqrt{3}$ & -0.00022 & \pm & 0.00001 & \multirow{2}{*}{59,850} \\
\hline & $+7.0 \mathrm{~mm}(7 \times$ limit $)$ & 0.00201 & \pm & 0.00016 & $7 \sqrt{3}$ & 0.00017 & \pm & 0.00001 & \\
\hline
\end{tabular}

\section{Packing Fraction}

Because of the overspecification of the TRISO particles in Table 1.14 (HTTR-GCR-RESR-001) and the correlation of uranium kernel diameter, density, TRISO packing fraction, and mass, the effect of the uncertainty in the packing fraction is not included in the total uncertainty. However, an analysis of the uncertainty based upon the fuel mass is performed in Section 2.1.6.

\subsubsection{Graphite Sleeves}

The uncertainty in the sleeve thickness was unreported. An inner diameter uncertainty of $\pm 0.5 \mathrm{~mm}$, which is limited by the $0.25 \mathrm{~mm}$ gap width between the sleeve and fuel compacts (Table 1.13 of HTTR-GCR-RESR-001), and an outer diameter uncertainty of $\pm 2 \mathrm{~mm}$ were assumed; their effects on the 
NEA/NSC/DOC(2006)1

\section{Gas Cooled (Thermal) Reactor - GCR \\ HTTR-GCR-RESR-002 \\ CRIT-REAC-RRATE}

uncertainty of $\mathrm{k}_{\mathrm{eff}}$ were determined. Figure 1.51 of HTTR-GCR-RESR-001 shows an inner diameter of $26.25 \mathrm{~mm}$ for the graphite sleeves, which would only provide a gap space of $0.125 \mathrm{~mm}$. The assumed uncertainty encompasses the discrepancy in this value. Results are shown in Tables 2.10 and 2.11.

The fuel sleeve height was varied $\pm 0.5 \mathrm{~mm}$ from the nominal value of $577 \mathrm{~mm}$ (Table 1.14 and Figure 1.51 of HTTR-GCR-RESR-001) and the effect on the uncertainty of $\mathrm{k}_{\mathrm{eff}}$ was determined. Results are shown in Table 2.12.

Later information was obtained regarding manufacturing tolerances for the fuel sleeves. The $\pm 0.1 \mathrm{~mm}$ of the ID and OD represents a bounding limit (with assumed uniform probability) and the height is then assumed to have a bounding limit (with assumed uniform probability) also of $\pm 0.1 \mathrm{~mm}$. ${ }^{\text {a }}$ The appropriate corrections to the scaling factors have been incorporated into the uncertainty analysis of these parameters.

The total number of graphite sleeves used in the fully-loaded core is 4,770. For determining the random component of the uncertainty, the results in Tables 2.10 through 2.12 would be divided by $\sqrt{ } \mathrm{N}$, where $\mathrm{N}$ for each case is shown in Tables 2.10 through 2.12 .

Table 2.10. Effect of Uncertainty in Graphite Sleeve Dimensions (Inner Diameter).

\begin{tabular}{|c|c|c|c|c|c|c|c|c|c|}
\hline Case & Deviation & $\Delta \mathrm{k}$ & \pm & $\sigma_{\Delta \mathrm{k}}$ & $\begin{array}{l}\text { Scaling } \\
\text { Factor } \\
\end{array}$ & $\Delta \mathrm{k}_{\mathrm{eff}}(1 \sigma)$ & \pm & $\sigma_{\Delta \mathrm{keff}}$ & $\mathrm{N}$ \\
\hline \multirow{2}{*}{1} & $-0.5 \mathrm{~mm}$ & 0.00079 & \pm & 0.00017 & $5 \sqrt{3}$ & 0.00009 & \pm & 0.00002 & \multirow{2}{*}{2,955} \\
\hline & $+0.5 \mathrm{~mm}$ & -0.00089 & \pm & 0.00017 & $5 \sqrt{3}$ & -0.00010 & \pm & 0.00002 & \\
\hline \multirow{2}{*}{2} & $-0.5 \mathrm{~mm}$ & 0.00077 & \pm & 0.00017 & $5 \sqrt{3}$ & 0.00009 & \pm & 0.00002 & \multirow{2}{*}{3,285} \\
\hline & $+0.5 \mathrm{~mm}$ & -0.00074 & \pm & 0.00016 & $5 \sqrt{3}$ & -0.00009 & \pm & 0.00002 & \\
\hline \multirow{2}{*}{3} & $-0.5 \mathrm{~mm}$ & 0.00083 & \pm & 0.00016 & $5 \sqrt{3}$ & 0.00010 & \pm & 0.00002 & \multirow{2}{*}{3,780} \\
\hline & $+0.5 \mathrm{~mm}$ & -0.00102 & \pm & 0.00016 & $5 \sqrt{3}$ & -0.00012 & \pm & 0.00002 & \\
\hline \multirow{2}{*}{4} & $-0.5 \mathrm{~mm}$ & 0.00111 & \pm & 0.00017 & $5 \sqrt{3}$ & 0.00013 & \pm & 0.00002 & \multirow{2}{*}{3,780} \\
\hline & $+0.5 \mathrm{~mm}$ & -0.00103 & \pm & 0.00017 & $5 \sqrt{3}$ & -0.00012 & \pm & 0.00002 & \\
\hline \multirow{2}{*}{5} & $-0.5 \mathrm{~mm}$ & 0.00110 & \pm & 0.00016 & $5 \sqrt{3}$ & 0.00013 & \pm & 0.00002 & \multirow{2}{*}{4,275} \\
\hline & $+0.5 \mathrm{~mm}$ & -0.00125 & \pm & 0.00016 & $5 \sqrt{3}$ & -0.00014 & \pm & 0.00002 & \\
\hline
\end{tabular}

\footnotetext{
${ }^{a}$ S. Maruyama, K. Yamashita, N. Fujimoto, I. Murata, R. Shindo, and Y. Sudo, "Determination of Hot Spot Factors for Calculation of the Maximum Fuel Temperatures in the Core Thermal and Hydraulic Design of HTTR," JAERIM 88-250, JAEA (November 18, 1988). [in Japanese].
} 
NEA/NSC/DOC(2006)1

\section{Gas Cooled (Thermal) Reactor - GCR \\ HTTR-GCR-RESR-002 \\ CRIT-REAC-RRATE}

Table 2.11. Effect of Uncertainty in Graphite Sleeve Dimensions (Outer Diameter).

\begin{tabular}{|c|c|c|c|c|c|c|c|c|c|}
\hline Case & Deviation & $\Delta \mathrm{k}$ & \pm & $\sigma_{\Delta \mathrm{k}}$ & $\begin{array}{c}\text { Scaling } \\
\text { Factor }\end{array}$ & $\Delta \mathrm{k}_{\mathrm{eff}}(1 \sigma)$ & \pm & $\sigma_{\Delta \mathrm{keff}}$ & $\mathrm{N}$ \\
\hline \multirow{2}{*}{1} & $-2 \mathrm{~mm}$ & -0.00499 & \pm & 0.00016 & $20 \sqrt{3}$ & -0.00014 & \pm & 0.00000 & \multirow{2}{*}{2,955} \\
\hline & $+2 \mathrm{~mm}$ & 0.00574 & \pm & 0.00016 & $20 \sqrt{3}$ & 0.00017 & \pm & 0.00000 & \\
\hline \multirow{2}{*}{2} & $-2 \mathrm{~mm}$ & -0.00555 & \pm & 0.00017 & $20 \sqrt{3}$ & -0.00016 & \pm & 0.00000 & \multirow{2}{*}{3,285} \\
\hline & $+2 \mathrm{~mm}$ & 0.00587 & \pm & 0.00017 & $20 \sqrt{3}$ & 0.00017 & \pm & 0000 & \\
\hline \multirow{2}{*}{3} & $-2 \mathrm{~mm}$ & -0.00622 & \pm & 0.00016 & $20 \sqrt{3}$ & -0.00018 & \pm & 0.00000 & \multirow{2}{*}{3,780} \\
\hline & $+2 \mathrm{~mm}$ & 0.00640 & \pm & 0.00016 & $20 \sqrt{3}$ & 0.00018 & \pm & 0.00000 & \\
\hline \multirow{2}{*}{4} & $-2 \mathrm{~mm}$ & -0.00692 & \pm & 0.00017 & $20 \sqrt{3}$ & -0.00020 & \pm & 0.00000 & \multirow{2}{*}{3,780} \\
\hline & $+2 \mathrm{~mm}$ & 0.00715 & \pm & 0.00017 & $20 \sqrt{3}$ & 0.00021 & \pm & 0.00000 & \\
\hline \multirow{2}{*}{5} & $-2 \mathrm{~mm}$ & -0.00675 & \pm & 0.00016 & $20 \sqrt{3}$ & -0.00019 & \pm & 0.00000 & \multirow{2}{*}{4,275} \\
\hline & $+2 \mathrm{~mm}$ & 0.00700 & \pm & 0.00016 & $20 \sqrt{3}$ & 0.00020 & \pm & 0.00000 & \\
\hline
\end{tabular}

Table 2.12. Effect of Uncertainty in Graphite Sleeve Dimensions (Height).

\begin{tabular}{|c|c|c|c|c|c|c|c|c|c|}
\hline Case & Deviation & $\Delta \mathrm{k}$ & \pm & $\sigma_{\Delta \mathrm{k}}$ & $\begin{array}{c}\text { Scaling } \\
\text { Factor }\end{array}$ & $\Delta \mathrm{k}_{\mathrm{eff}}(1 \sigma)$ & \pm & $\sigma_{\Delta \mathrm{keff}}$ & $\mathrm{N}$ \\
\hline \multirow{2}{*}{1} & $-0.5 \mathrm{~mm}(1 \sigma)$ & -0.00047 & \pm & 0.00016 & $5 \sqrt{3}$ & -0.00005 & \pm & 0.00002 & \multirow{2}{*}{2,955} \\
\hline & $+0.5 \mathrm{~mm}(1 \sigma)$ & 0.00039 & \pm & 0.00017 & $5 \sqrt{3}$ & 0.00005 & \pm & 0.00002 & \\
\hline \multirow{2}{*}{2} & $-0.5 \mathrm{~mm}(1 \sigma)$ & -0.00042 & \pm & 0.00017 & $5 \sqrt{3}$ & -0.00005 & \pm & 0.00002 & \multirow{2}{*}{3,285} \\
\hline & $+0.5 \mathrm{~mm}(1 \sigma)$ & 0.00036 & \pm & 0.00016 & $5 \sqrt{3}$ & 0.00004 & \pm & 0.00002 & \\
\hline \multirow{2}{*}{3} & $-0.5 \mathrm{~mm}(1 \sigma)$ & -0.00035 & \pm & 0.00017 & $5 \sqrt{3}$ & -0.00004 & \pm & 0.00002 & \multirow{2}{*}{3,780} \\
\hline & $+0.5 \mathrm{~mm}(1 \sigma)$ & 0.00025 & \pm & 0.00016 & $5 \sqrt{3}$ & 0.00003 & \pm & 0.00002 & \\
\hline \multirow{2}{*}{4} & $-0.5 \mathrm{~mm}(1 \sigma)$ & -0.00058 & \pm & 0.00017 & $5 \sqrt{3}$ & -0.00007 & \pm & 0.00002 & \multirow{2}{*}{3,780} \\
\hline & $+0.5 \mathrm{~mm}(1 \sigma)$ & 0.00026 & \pm & 0.00017 & $5 \sqrt{3}$ & 0.00003 & \pm & 0.00002 & \\
\hline \multirow{2}{*}{5} & $-0.5 \mathrm{~mm}(1 \sigma)$ & -0.00068 & \pm & 0.00016 & $5 \sqrt{3}$ & -0.00008 & \pm & 0.00002 & \multirow{2}{*}{4,275} \\
\hline & $+0.5 \mathrm{~mm}(1 \sigma)$ & 0.00022 & \pm & 0.00016 & $5 \sqrt{3}$ & 0.00003 & \pm & 0.00002 & \\
\hline
\end{tabular}

\subsubsection{Burnable Poisons}

The uncertainty in the diameters of the BPs and BP insertion holes was unreported. A BP diameter uncertainty of $\pm 1 \mathrm{~mm}$ from the nominal diameter (D) of $14 \mathrm{~mm}$ and a BP insertion hole diameter uncertainty of $\pm 1 \mathrm{~mm}$ from the nominal diameter of $15 \mathrm{~mm}$ were assumed and their effects on the uncertainty of $\mathrm{k}_{\mathrm{eff}}$ were determined. Results are shown in Tables 2.13 and 2.15.

The uncertainty in the height stack of the BPs was unreported. A stack height (H) uncertainty of $\pm 1 \mathrm{~mm}$ from the nominal height of $200 \mathrm{~mm}$ was assumed (approximately $\pm 0.1 \mathrm{~mm}$ per BP pellet) and the effective uncertainty in $k_{\text {eff }}$ was determined. The height of the BP insertion hole was adjusted to accommodate the change in BP stack height. Results are shown in Table 2.14. 
NEA/NSC/DOC(2006)1

\section{Gas Cooled (Thermal) Reactor - GCR \\ HTTR-GCR-RESR-002 \\ CRIT-REAC-RRATE}

The uncertainty in the dimensions of the graphite disks was unreported. A diameter uncertainty of \pm 1 $\mathrm{mm}$ from the nominal diameter of $14 \mathrm{~mm}$ and a height uncertainty of $\pm 1 \mathrm{~mm}$ from the nominal stack height of $100 \mathrm{~mm}$ were assumed and their effects on the uncertainty of $\mathrm{k}_{\text {eff }}$ were determined. The height of the BP insertion hole was adjusted to accommodate the change in graphite-disk stack height. Results are shown in Tables 2.16 and 2.17.

Because of the tight manufacturing tolerances of the fuel compacts and graphite sleeves (Sections 2.1.2.2 and 2.1.2.3, respectively) it is believed that similar tolerances apply to other graphite and boron carbide components of the HTTR. Therefore, the BP and graphite disk diameters are treated with a tolerance (with uniform probability) of $\pm 0.1 \mathrm{~mm}$ and a stack height tolerance (with uniform probability) of \pm 1.0 $\mathrm{mm}$. The appropriate corrections to the scaling factors have been incorporated into the uncertainty analysis of these parameters.

The total number of burnable poison pellets used in the fully-loaded core is 5,520. For determining the random component of the uncertainty, the results in Tables 2.13 through 2.17 would be divided by $\sqrt{\mathrm{N}}$, where $\mathrm{N}$ for each case is shown in Tables 2.13 through 2.17. The total number of BP stacks is 600 , of $\mathrm{BP}$ insertion holes is 450 , and of graphite disk stacks is 300 , in the fully-loaded core configuration.

Table 2.13. Effect of Uncertainty in BP Pin Dimensions (Diameter).

\begin{tabular}{||c|c|ccc|c|ccc|c||}
\hline \hline Case & Deviation & $\Delta \mathrm{k}$ & \pm & $\sigma_{\Delta \mathrm{k}}$ & $\begin{array}{c}\text { Scaling } \\
\text { Factor }\end{array}$ & $\Delta \mathrm{k}_{\text {eff }}(1 \sigma)$ & \pm & $\sigma_{\Delta \mathrm{keff}}$ & $\mathrm{N}$ \\
\hline \hline \multirow{2}{*}{1} & $-1 \mathrm{~mm}(10 \times$ limit $)$ & 0.01047 & \pm & 0.00017 & $10 \sqrt{3}$ & 0.00060 & \pm & 0.00001 & \multirow{2}{*}{3,496} \\
& $+1 \mathrm{~mm}(10 \times$ limit $)$ & -0.00974 & \pm & 0.00017 & $10 \sqrt{3}$ & -0.00056 & \pm & 0.00001 & \\
\hline \multirow{2}{*}{2} & $-1 \mathrm{~mm}(10 \times$ limit $)$ & 0.01125 & \pm & 0.00016 & $10 \sqrt{3}$ & 0.00065 & \pm & 0.00001 & \\
& $+1 \mathrm{~mm}(10 \times$ limit $)$ & -0.01045 & \pm & 0.00016 & $10 \sqrt{3}$ & -0.00060 & \pm & 0.00001 & 3,864 \\
\hline \multirow{2}{*}{3} & $-1 \mathrm{~mm}(10 \times$ limit $)$ & 0.01198 & \pm & 0.00016 & $10 \sqrt{3}$ & 0.00069 & \pm & 0.00001 & \\
& $+1 \mathrm{~mm}(10 \times$ limit $)$ & -0.01149 & \pm & 0.00016 & $10 \sqrt{3}$ & -0.00066 & \pm & 0.00001 & 4,416 \\
\hline \multirow{2}{*}{4} & $-1 \mathrm{~mm}(10 \times$ limit $)$ & 0.01202 & \pm & 0.00017 & $10 \sqrt{3}$ & 0.00069 & \pm & 0.00001 & \\
& $+1 \mathrm{~mm}(10 \times$ limit $)$ & -0.01171 & \pm & 0.00016 & $10 \sqrt{3}$ & -0.00068 & \pm & 0.00001 & 4,416 \\
\hline \multirow{2}{*}{5} & $-1 \mathrm{~mm}(10 \times$ limit $)$ & 0.01287 & \pm & 0.00016 & $10 \sqrt{3}$ & 0.00074 & \pm & 0.00001 & \\
& $+1 \mathrm{~mm}(10 \times$ limit $)$ & -0.01234 & \pm & 0.00016 & $10 \sqrt{3}$ & -0.00071 & \pm & 0.00001 & 4,968 \\
\hline \hline
\end{tabular}


NEA/NSC/DOC(2006)1

Gas Cooled (Thermal) Reactor - GCR

HTTR-GCR-RESR-002

CRIT-REAC-RRATE

Table 2.14. Effect of Uncertainty in BP Pin Dimensions (Stack Height).

\begin{tabular}{|c|c|c|c|c|c|c|c|c|c|}
\hline Case & Deviation & $\Delta \mathrm{k}$ & \pm & $\sigma_{\Delta \mathrm{k}}$ & $\begin{array}{l}\text { Scaling } \\
\text { Factor }\end{array}$ & $\Delta \mathrm{k}_{\mathrm{eff}}(1 \sigma)$ & \pm & $\sigma_{\Delta \mathrm{keff}}$ & $\mathrm{N}$ \\
\hline \multirow{2}{*}{1} & $-1 \mathrm{~mm}$ & 0.00061 & \pm & 0.00017 & $\sqrt{3}$ & 0.00035 & \pm & 0.00010 & \multirow{2}{*}{380} \\
\hline & $+1 \mathrm{~mm}$ & -0.00050 & \pm & 0.00017 & $\sqrt{3}$ & -0.00029 & \pm & 0.00010 & \\
\hline \multirow{2}{*}{2} & $-1 \mathrm{~mm}$ & 0.00056 & \pm & 0.00017 & $\sqrt{3}$ & 0.00032 & \pm & 0.00010 & \multirow{2}{*}{420} \\
\hline & $+1 \mathrm{~mm}$ & -0.00065 & + & 0.00016 & $\sqrt{3}$ & -0.00038 & \pm & 0.00009 & \\
\hline \multirow{2}{*}{3} & $-1 \mathrm{~mm}$ & 0.00044 & \pm & 0.00017 & $\sqrt{3}$ & 0.00025 & \pm & 0.00010 & \multirow{2}{*}{480} \\
\hline & $+1 \mathrm{~mm}$ & -0.00077 & - & 0.00017 & $\sqrt{3}$ & -0.00044 & \pm & 0.00010 & \\
\hline \multirow{2}{*}{4} & $-1 \mathrm{~mm}$ & 0.00050 & \pm & 0.00017 & $\sqrt{3}$ & 0.00029 & \pm & 0.00010 & \multirow{2}{*}{480} \\
\hline & $+1 \mathrm{~mm}$ & -0.00088 & \pm & 0.00017 & $\sqrt{3}$ & -0.00051 & \pm & 0.00010 & \\
\hline \multirow{2}{*}{5} & $-1 \mathrm{~mm}$ & 0.00055 & \pm & 0.00016 & $\sqrt{3}$ & 0.00032 & \pm & 0.00009 & \multirow{2}{*}{520} \\
\hline & $+1 \mathrm{~mm}$ & -0.00065 & \pm & 0.00016 & $\sqrt{3}$ & -0.00038 & \pm & 0.00009 & \\
\hline
\end{tabular}

Table 2.15. Effect of Uncertainty in BP Insertion Hole Diameter.

\begin{tabular}{|c|c|c|c|c|c|c|c|c|c|}
\hline Case & Deviation & $\Delta \mathrm{k}$ & \pm & $\sigma_{\Delta \mathrm{k}}$ & $\begin{array}{l}\text { Scaling } \\
\text { Factor }\end{array}$ & $\Delta \mathrm{k}_{\mathrm{eff}}(1 \sigma)$ & \pm & $\sigma_{\Delta \mathrm{keff}}$ & $\mathrm{N}$ \\
\hline \multirow{2}{*}{1} & $-1 \mathrm{~mm}(10 \times$ limit $)$ & 0.00102 & \pm & 0.00017 & $10 \sqrt{3}$ & 0.00006 & + & 0.00001 & \multirow{2}{*}{285} \\
\hline & $+1 \mathrm{~mm}(10 \times$ limit $)$ & -0.00092 & \pm & 0.00017 & $10 \sqrt{3}$ & -0.00005 & \pm & 0.00001 & \\
\hline \multirow{2}{*}{2} & $-1 \mathrm{~mm}(10 \times$ limit $)$ & 0.00116 & \pm & 0.00016 & $10 \sqrt{3}$ & 0.00007 & \pm & 0.00001 & \multirow{2}{*}{315} \\
\hline & $+1 \mathrm{~mm}(10 \times$ limit $)$ & -0.00076 & \pm & 0.00016 & $10 \sqrt{3}$ & -0.00004 & \pm & 0.00001 & \\
\hline \multirow{2}{*}{3} & $-1 \mathrm{~mm}(10 \times$ limit $)$ & 0.00076 & \pm & 0.00017 & $10 \sqrt{3}$ & 0.00004 & $\perp$ & 0.00001 & \multirow{2}{*}{360} \\
\hline & $+1 \mathrm{~mm}(10 \times$ limit $)$ & -0.00104 & \pm & 0.00016 & $10 \sqrt{3}$ & -0.00006 & & 0.00001 & \\
\hline \multirow{2}{*}{4} & $-1 \mathrm{~mm}(10 \times$ limit $)$ & 0.00069 & \pm & 0.00016 & $10 \sqrt{3}$ & 0.00004 & & 0.00001 & \multirow{2}{*}{360} \\
\hline & $+1 \mathrm{~mm}(10 \times$ limit $)$ & -0.00122 & \pm & 0.00016 & $10 \sqrt{3}$ & -0.00007 & \pm & 0.00001 & \\
\hline \multirow{2}{*}{5} & $-1 \mathrm{~mm}(10 \times$ limit $)$ & 0.00109 & \pm & 0.00016 & $10 \sqrt{3}$ & 0.00006 & & 0.00001 & \multirow{2}{*}{405} \\
\hline & $+1 \mathrm{~mm}(10 \times$ limit $)$ & -0.00098 & \pm & 0.00016 & $10 \sqrt{3}$ & -0.00006 & \pm & 0.00001 & \\
\hline
\end{tabular}


NEA/NSC/DOC(2006)1

Gas Cooled (Thermal) Reactor - GCR

HTTR-GCR-RESR-002

CRIT-REAC-RRATE

Table 2.16. Effect of Uncertainty in Graphite Disk Dimensions (Diameter).

\begin{tabular}{|c|c|c|c|c|c|c|c|c|c|}
\hline Case & Deviation & $\Delta \mathrm{k}$ & & $\sigma_{\Delta \mathrm{k}}$ & $\begin{array}{c}\text { Scaling } \\
\text { Factor }\end{array}$ & $\Delta \mathrm{k}_{\mathrm{eff}}(1 \sigma)$ & & $\sigma_{\Delta \mathrm{keff}}$ & $\mathrm{N}$ \\
\hline \multirow{2}{*}{1} & & & & & & & & & \multirow{2}{*}{190} \\
\hline & $+1 n$ & 0.00008 & & 0.00017 & $10 \sqrt{3}$ & 000 & & 0.00001 & \\
\hline \multirow{2}{*}{2} & $(10 \times$ limit $)$ & & & 16 & $10 \sqrt{3}$ & & & 01 & \multirow{2}{*}{210} \\
\hline & $+1 \mathrm{n}$ & -0 & & 17 & $10 \sqrt{3}$ & 0 & & 01 & \\
\hline \multirow{2}{*}{3} & nit) & -0.00002 & + & 0.00016 & $10 \sqrt{3}$ & 0.00000 & & 0.00001 & \multirow{2}{*}{240} \\
\hline & $+1 \mathrm{~m}$ & 0.00011 & & 0.00017 & $10 \sqrt{3}$ & 00 & & 01 & \\
\hline \multirow{2}{*}{2} & $-1 \mathrm{mn}$ & -0.0 & & 0.00017 & $10 \sqrt{3}$ & -0.00001 & & 0.00001 & \multirow{2}{*}{240} \\
\hline & $+1 \mathrm{~m}$ & -0.00008 & & 017 & $10 \sqrt{3}$ & & & 001 & \\
\hline \multirow{2}{*}{5} & $-1 \mathrm{~mm}(10 \times$ limit $)$ & -0.00004 & & 0.00016 & $10 \sqrt{3}$ & 0.00000 & & 0.00001 & \multirow{2}{*}{270} \\
\hline & $+1 \mathrm{~mm}(10 \times$ limit $)$ & -0.00012 & \pm & 0.00016 & $10 \sqrt{3}$ & -0.00001 & & 0.00001 & \\
\hline
\end{tabular}

Table 2.17. Effect of Uncertainty in Graphite Disk Dimensions (Stack Height).

\begin{tabular}{||c|c|ccc|c|ccc|c||}
\hline \hline Case & Deviation & $\Delta \mathrm{k}$ & \pm & $\sigma_{\Delta \mathrm{k}}$ & $\begin{array}{c}\text { Scaling } \\
\text { Factor }\end{array}$ & $\Delta \mathrm{k}_{\text {eff }}(1 \sigma)$ & \pm & $\sigma_{\Delta \mathrm{keff}}$ & $\mathrm{N}$ \\
\hline \multirow{2}{*}{1} & $-1 \mathrm{~mm}$ & -0.00001 & \pm & 0.00017 & $\sqrt{3}$ & -0.00001 & \pm & 0.00010 & \multirow{2}{*}{190} \\
& $+1 \mathrm{~mm}$ & 0.00022 & \pm & 0.00016 & $\sqrt{ } 3$ & 0.00013 & \pm & 0.00009 & \\
\hline \multirow{2}{*}{2} & $-1 \mathrm{~mm}$ & 0.00008 & \pm & 0.00016 & $\sqrt{3}$ & 0.00005 & \pm & 0.00009 & \multirow{2}{*}{210} \\
& $+1 \mathrm{~mm}$ & -0.00005 & \pm & 0.00017 & $\sqrt{3}$ & -0.00003 & \pm & 0.00010 & \\
\hline \multirow{3}{*}{3} & $-1 \mathrm{~mm}$ & -0.00004 & \pm & 0.00016 & $\sqrt{3}$ & -0.00002 & \pm & 0.00009 & \multirow{2}{*}{240} \\
& $+1 \mathrm{~mm}$ & 0.00001 & \pm & 0.00017 & $\sqrt{3}$ & 0.00001 & \pm & 0.00010 & \\
4 & $-1 \mathrm{~mm}$ & 0.00000 & \pm & 0.00017 & $\sqrt{3}$ & 0.00000 & \pm & 0.00010 & \multirow{2}{*}{4} \\
& $+1 \mathrm{~mm}$ & -0.00009 & \pm & 0.00017 & $\sqrt{3}$ & -0.00005 & \pm & 0.00010 & \\
\hline \multirow{2}{*}{5} & $-1 \mathrm{~mm}$ & -0.00015 & \pm & 0.00016 & $\sqrt{3}$ & -0.00009 & \pm & 0.00009 & 270 \\
& $+1 \mathrm{~mm}$ & 0.00021 & \pm & 0.00016 & $\sqrt{3}$ & 0.00012 & \pm & 0.00009 & \\
\hline \hline
\end{tabular}

\subsubsection{Control Rods}

\section{Absorber Dimensions}

The uncertainty in the absorber dimensions was unreported. Assumed inner and outer diameter (ID and OD) uncertainties are $\pm 5 \mathrm{~mm}$ each from the nominal values of 75 and $105 \mathrm{~mm}$ (Table 1.15 of HTTR-GCR-RESR-001), respectively, and their effects on the uncertainty of $\mathrm{k}_{\text {eff }}$ were determined. The uncertainty in the pellet height was assumed to be $\pm 1 \mathrm{~cm}$ from the nominal stack height $(\mathrm{H})$ of $29 \mathrm{~cm}$. The alternate value of $115 \mathrm{~mm}$ provided for the outer diameter of the absorber material is believed to be a typographical error (see footnote of Table 1.15 of HTTR-GCR-RESR-001). Results are shown in Tables 2.18 through 2.20 . 
NEA/NSC/DOC(2006)1

\section{Gas Cooled (Thermal) Reactor - GCR \\ HTTR-GCR-RESR-002 \\ CRIT-REAC-RRATE}

Because of the tight manufacturing tolerances of the fuel compacts and graphite sleeves (Sections 2.1.2.2 and 2.1.2.3, respectively) it is believed that similar tolerances apply to other graphite and boron carbide components of the HTTR. Therefore, the absorber diameters are treated with a tolerance (with uniform probability) of $\pm 0.1 \mathrm{~mm}$ and a stack height tolerance (with uniform probability) of $\pm 1.0 \mathrm{~mm}$. The appropriate corrections to the scaling factors have been incorporated into the uncertainty analysis of these parameters.

The total number of control rod absorber pellets used in the fully-loaded core is approximately 975 , as approximately $30 \%$ of the control rods are actually inserted into the core. For determining the random component of the uncertainty, the results in Tables 2.18 through 2.20 would be divided by $\sqrt{ } \mathrm{N}$, where $\mathrm{N}$ for each case is shown in Tables 2.18 through 2.20.

Table 2.18. Effect of Uncertainty in CR Absorber Dimensions (Inner Diameter).

\begin{tabular}{|c|c|ccc|c|ccc|c||}
\hline Case & Deviation & $\Delta \mathrm{k}$ & \pm & $\sigma_{\Delta \mathrm{k}}$ & $\begin{array}{c}\text { Scaling } \\
\text { Factor }\end{array}$ & $\Delta \mathrm{k}_{\text {eff }}(1 \sigma)$ & \pm & $\sigma_{\Delta \mathrm{keff}}$ & $\mathrm{N}$ \\
\hline \hline \multirow{2}{*}{1} & $-5 \mathrm{~mm}(50 \times$ limit $)$ & -0.00026 & \pm & 0.00017 & $50 \sqrt{3}$ & 0.00000 & \pm & 0.00000 & \\
& $+5 \mathrm{~mm}(50 \times$ limit $)$ & 0.00003 & \pm & 0.00017 & $50 \sqrt{3}$ & 0.00000 & \pm & 0.00000 & \\
\hline \multirow{2}{*}{2} & $-5 \mathrm{~mm}(50 \times$ limit $)$ & -0.00021 & \pm & 0.00017 & $50 \sqrt{3}$ & 0.00000 & \pm & 0.00000 & \\
& $+5 \mathrm{~mm}(50 \times$ limit $)$ & 0.00037 & \pm & 0.00017 & $50 \sqrt{3}$ & 0.00000 & \pm & 0.00000 & 585 \\
\hline \multirow{3}{*}{3} & $-5 \mathrm{~mm}(50 \times$ limit $)$ & -0.00033 & \pm & 0.00017 & $50 \sqrt{3}$ & 0.00000 & \pm & 0.00000 & \\
& $+5 \mathrm{~mm}(50 \times$ limit $)$ & 0.00014 & \pm & 0.00017 & $50 \sqrt{3}$ & 0.00000 & \pm & 0.00000 & \\
\hline \multirow{3}{*}{4} & $-5 \mathrm{~mm}(50 \times$ limit $)$ & -0.00042 & \pm & 0.00016 & $50 \sqrt{3}$ & 0.00000 & \pm & 0.00000 & \\
& $+5 \mathrm{~mm}(50 \times$ limit $)$ & 0.00037 & \pm & 0.00017 & $50 \sqrt{3}$ & 0.00000 & \pm & 0.00000 & 720 \\
\hline \multirow{2}{*}{5} & $-5 \mathrm{~mm}(50 \times$ limit $)$ & -0.00032 & \pm & 0.00016 & $50 \sqrt{3}$ & 0.00000 & \pm & 0.00000 & \\
& $+5 \mathrm{~mm}(50 \times$ limit $)$ & 0.00035 & \pm & 0.00016 & $50 \sqrt{3}$ & 0.00000 & \pm & 0.00000 & 910 \\
\hline \hline
\end{tabular}

Table 2.19. Effect of Uncertainty in CR Absorber Dimensions (Outer Diameter).

\begin{tabular}{|c|c|c|c|c|c|c|c|c|c|}
\hline Case & Devi & $\Delta \mathrm{k}$ & & $\sigma_{\Delta \mathrm{k}}$ & $\begin{array}{l}\text { Scaling } \\
\text { Factor }\end{array}$ & $\Delta \mathrm{k}_{\mathrm{eff}}(1 \sigma)$ & \pm & $\sigma_{\Delta \mathrm{keff}}$ & $\mathrm{N}$ \\
\hline & & & & & $\pi$ & 1 & \pm & 0.0 & \multirow{2}{*}{225} \\
\hline & $+5 \mathrm{n}$ & -0.00083 & & 0.00017 & $50 \sqrt{3}$ & -0.00001 & \pm & 000 & \\
\hline \multirow{2}{*}{2} & $-5 r$ & 0.0 & & 0.0 & $50 \sqrt{3}$ & 02 & \pm & 00 & \multirow{2}{*}{585} \\
\hline & $+5 n$ & -0.0 & & 0.00016 & $50 \sqrt{3}$ & 11 & \pm & 0 & \\
\hline \multirow[b]{2}{*}{3} & $-5 n$ & 0.00135 & & 0.00017 & $50 \sqrt{3}$ & 0.00002 & \pm & 0.00000 & \multirow{2}{*}{780} \\
\hline & $+5 \mathrm{n}$ & -0.0 & & 0.00017 & $50 \sqrt{3}$ & -0 . & \pm & & \\
\hline & $(50 \times$ limit $)$ & 0.00226 & & 0.00017 & $50 \sqrt{3}$ & 0.00003 & \pm & 0.00000 & \multirow{2}{*}{720} \\
\hline & $+5 \mathrm{~mm}(50$ & -0.00275 & & 0.00017 & $50 \sqrt{3}$ & -0.00003 & \pm & 000 & \\
\hline & $-5 \mathrm{~mm}(50 \times$ limit $)$ & 0.00160 & & 0.00016 & $50 \sqrt{3}$ & 0.00002 & \pm & 0.00000 & \multirow[b]{2}{*}{910} \\
\hline & $+5 \mathrm{~mm}(50 \times$ limit $)$ & -0.00187 & & 0.00016 & $50 \sqrt{3}$ & -0.00002 & \pm & 0.00000 & \\
\hline
\end{tabular}


NEA/NSC/DOC(2006)1

Gas Cooled (Thermal) Reactor - GCR

HTTR-GCR-RESR-002

CRIT-REAC-RRATE

Table 2.20. Effect of Uncertainty in CR Absorber Dimensions (Stack Height).

\begin{tabular}{|c|c|c|c|c|c|c|c|c|c|}
\hline Case & Devi & $\Delta \mathrm{k}$ & \pm & $\sigma_{\Delta \mathrm{k}}$ & $\begin{array}{l}\text { Scaling } \\
\text { Factor }\end{array}$ & $\Delta \mathrm{k}_{\mathrm{eff}}(1 \sigma)$ & \pm & $\sigma_{\Delta \mathrm{keff}}$ & $\mathrm{N}$ \\
\hline \multirow{2}{*}{1} & & & \pm & & $10 \sqrt{3}$ & & \pm & & \multirow{2}{*}{22.5} \\
\hline & $+1 \mathrm{~cm}(10 \times$ & -0.00023 & 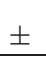 & 0.00016 & $10 \sqrt{3}$ & -0.00001 & \pm & 0.00001 & \\
\hline \multirow{2}{*}{2} & & & \pm & & $10 \sqrt{3}$ & & \pm & & \multirow{2}{*}{58.5} \\
\hline & $+1 \mathrm{~cm}(10$ & -0. & 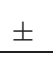 & 0. & $10 \sqrt{3}$ & -0.0 & \pm & 1 & \\
\hline \multirow[b]{2}{*}{3} & $-1 \mathrm{cr}$ & 0. & . & 0. & $10 \sqrt{3}$ & 0.00002 & \pm & 01 & \multirow{2}{*}{78.0} \\
\hline & $+1 \mathrm{~cm} \mathrm{(1}$ & -0.00099 & - & 0.00017 & $10 \sqrt{3}$ & -0.00006 & \pm & 1 & \\
\hline \multirow[b]{2}{*}{4} & $0 \times$ limit) & 0.00059 & \pm & 0.00017 & $10 \sqrt{3}$ & 0.00003 & \pm & 0.00001 & \multirow{2}{*}{72.0} \\
\hline & $+1 \mathrm{~cm}(10 \times$ limit $)$ & -0.00073 & \pm & 0.00017 & $10 \sqrt{3}$ & -0.00004 & \pm & 0.00001 & \\
\hline & $-1 \mathrm{~cm}(10 \times$ limit $)$ & 0.00042 & + & 0.00016 & $10 \sqrt{3}$ & 0.00002 & \pm & 0.00001 & \multirow{2}{*}{91.0} \\
\hline & $+1 \mathrm{~cm}(10 \times$ limit $)$ & -0.00111 & \pm & 0.00016 & $10 \sqrt{3}$ & -0.00006 & \pm & 0.00001 & \\
\hline
\end{tabular}

\section{Clad Dimensions}

The uncertainty in the clad dimensions was unreported, and detailed dimensions for anything similar to the diagram shown in Figure 1.60 of HTTR-GCR-RESR-001were unavailable. Therefore a solid clad material encasing the control rod absorber was defined without detail for the end caps. Inner and outer thicknesses were varied $\pm 5 \mathrm{~mm}$ from the nominal values of 65 and $113 \mathrm{~mm}$ (Table 1.15 of HTTR-GCR-RESR-001), respectively, to determine their effects on the uncertainty of $k_{\text {eff. }}$ The spine diameter was varied $\pm 10 \mathrm{~mm}$ from the nominal value of $10 \mathrm{~mm}$ (Table 1.15 of HTTR-GCR-RESR-001) and its effect on the uncertainty of $\mathrm{k}_{\text {eff }}$ was determined. The clad height of a single control rod section was varied $\pm 1 \mathrm{~cm}$. The reported clad thickness of $3.5 \mathrm{~mm}$ does not appear to conform to the difference between reported diameters of the clad and absorber materials. It is unclear whether a gap exists or there is rounding of values in the original table. The uncertainties evaluated encompass the overall uncertainty in this discrepancy. The control rod diameters are chosen as the more appropriate dimensions for modeling, such that inner and outer clad thicknesses are approximately 5 and $4 \mathrm{~mm}$, respectively. Results are shown in Tables 2.21 through 2.24 .

Height change of clad also affects the effective height of each control rod segment.

Because of the tight manufacturing tolerances of the fuel compacts and graphite sleeves (Sections 2.1.2.2 and 2.1.2.3, respectively) it is believed that tighter tolerances apply to the absorber cladding. Therefore, the diameters are treated with a tolerance (with uniform probability) of $\pm 0.5 \mathrm{~mm}$ and a height tolerance (with uniform probability) of $\pm 1.0 \mathrm{~mm}$. The appropriate corrections to the scaling factors have been incorporated into the uncertainty analysis of these parameters.

The total number of control rod sections used in the fully-loaded core is approximately 97.5, as approximately $30 \%$ of the control rods are actually inserted into the core. For determining the random component of the uncertainty, the results in Tables 2.21 through 2.24 would be divided by $\sqrt{ } \mathrm{N}$, where $\mathrm{N}$ for each case is shown in Tables 2.21 through 2.24. 
NEA/NSC/DOC(2006)1

Gas Cooled (Thermal) Reactor - GCR

HTTR-GCR-RESR-002

CRIT-REAC-RRATE

Table 2.21. Effect of Uncertainty in CR Clad Dimensions (Inner Diameter).

\begin{tabular}{|c|c|c|c|c|c|c|c|c|c|}
\hline Case & Deviation & $\Delta \mathrm{k}$ & \pm & $\sigma_{\Delta \mathrm{k}}$ & $\begin{array}{l}\text { Scaling } \\
\text { Factor }\end{array}$ & $\Delta \mathrm{k}_{\mathrm{eff}}(1 \sigma)$ & \pm & $\sigma_{\Delta \mathrm{keff}}$ & $\mathrm{N}$ \\
\hline \multirow{2}{*}{1} & & & \pm & & & & \pm & & \multirow{2}{*}{22.5} \\
\hline & $+5 \mathrm{~mm}(10 \times$ & -0.00018 & + & 0.00017 & $10 \sqrt{3}$ & -0.00001 & \pm & 0.00001 & \\
\hline \multirow{2}{*}{2} & $-5 \mathrm{~mm}(10 \times$ limit $)$ & -0.0 & \pm & 0.00017 & $10 \sqrt{3}$ & & \pm & 01 & \multirow{2}{*}{58.5} \\
\hline & $+5 \mathrm{n}$ & 0.0 & - & & $10 \sqrt{3}$ & & $\perp$ & & \\
\hline \multirow{2}{*}{3} & $-5 \mathrm{~mm}(10 \times 1 \mathrm{i}$ & -0.00003 & \pm & 0.00016 & $10 \sqrt{3}$ & 0.00000 & \pm & 0.00001 & \multirow{2}{*}{78.0} \\
\hline & $+5 \mathrm{~m}$ & -0.0 & - & 0.00011 & $10 \sqrt{3}$ & 01 & $\perp$ & 01 & \\
\hline \multirow{2}{*}{4} & $-5 \mathrm{~mm}(10 \times$ limit $)$ & -0.00007 & \pm & 0.00017 & $10 \sqrt{3}$ & 0.00000 & I & 0.00001 & \multirow{2}{*}{72.0} \\
\hline & $+5 \mathrm{~mm}(10 \times$ limit $)$ & -0.00011 & $I$ & 0.00017 & $10 \sqrt{3}$ & -0.00001 & \pm & 001 & \\
\hline & $-5 \mathrm{~mm}(10 \times$ limit $)$ & -0.00002 & \pm & 0.00016 & $10 \sqrt{3}$ & 0.00000 & \pm & 0.00001 & \multirow{2}{*}{91.0} \\
\hline & it) & -0.00006 & \pm & 0016 & $10 \sqrt{3}$ & 0.00000 & \pm & 0.00001 & \\
\hline
\end{tabular}

Table 2.22. Effect of Uncertainty in CR Clad Dimensions (Outer Diameter).

\begin{tabular}{|c|c|c|c|c|c|c|c|c|c|}
\hline Case & Deviation & $\Delta \mathrm{k}$ & \pm & $\sigma_{\Delta \mathrm{k}}$ & $\begin{array}{l}\text { Scaling } \\
\text { Factor }\end{array}$ & $\Delta \mathrm{k}_{\mathrm{eff}}(1 \sigma)$ & \pm & $\sigma_{\Delta \mathrm{keff}}$ & $\mathrm{N}$ \\
\hline \multirow{2}{*}{1} & $-5 \mathrm{~mm}(10 \times$ limit $)$ & -0.00033 & \pm & 0.00017 & $10 \sqrt{3}$ & -0.00002 & \pm & 0.00001 & \multirow{2}{*}{22.5} \\
\hline & $+5 \mathrm{~mm}(10 \times$ limit $)$ & 0.00018 & \pm & 0.00017 & $10 \sqrt{3}$ & 0.00001 & \pm & 0.00001 & \\
\hline \multirow{2}{*}{2} & $-5 \mathrm{~mm}(10 \times$ limit $)$ & -0.00053 & \pm & 0.00017 & $10 \sqrt{3}$ & -0.00003 & \pm & 0.00001 & \multirow{2}{*}{58.5} \\
\hline & $+5 \mathrm{~mm}(10 \times$ limit $)$ & 0.00028 & \pm & 0.00016 & $10 \sqrt{3}$ & 0.00002 & \pm & 0.00001 & \\
\hline \multirow{2}{*}{3} & $-5 \mathrm{~mm}(10 \times$ limit $)$ & -0.00082 & \pm & 0.00017 & $10 \sqrt{3}$ & -0.00005 & \pm & 0.00001 & \multirow{2}{*}{78.0} \\
\hline & $+5 \mathrm{~mm}(10 \times$ limit $)$ & 0.00055 & \pm & 0.00016 & $10 \sqrt{3}$ & 0.00003 & \pm & 0.00001 & \\
\hline \multirow{2}{*}{4} & $-5 \mathrm{~mm}(10 \times$ limit $)$ & -0.00169 & \pm & 0.00017 & $10 \sqrt{3}$ & -0.00010 & \pm & 0.00001 & \multirow{2}{*}{72.0} \\
\hline & $+5 \mathrm{~mm}(10 \times$ limit $)$ & 0.00108 & \pm & 0.00017 & $10 \sqrt{3}$ & 0.00006 & \pm & 0.00001 & \\
\hline & $-5 \mathrm{~mm}(10 \times$ limit $)$ & -0.00087 & \pm & 0.00016 & $10 \sqrt{3}$ & -0.00005 & \pm & 0.00001 & \multirow{2}{*}{91.0} \\
\hline & $+5 \mathrm{~mm}(10 \times$ limit $)$ & 0.00058 & \pm & 0.00016 & $10 \sqrt{3}$ & 0.00003 & \pm & 0.00001 & \\
\hline
\end{tabular}


NEA/NSC/DOC(2006)1

Gas Cooled (Thermal) Reactor - GCR

HTTR-GCR-RESR-002

CRIT-REAC-RRATE

Table 2.23. Effect of Uncertainty in CR Clad Dimensions (Height).

\begin{tabular}{||c|c|ccc|c|ccc|c||}
\hline Case & Deviation & $\Delta \mathrm{k}$ & \pm & $\sigma_{\Delta \mathrm{k}}$ & $\begin{array}{c}\text { Scaling } \\
\text { Factor }\end{array}$ & $\Delta \mathrm{k}_{\text {eff }}(1 \sigma)$ & \pm & $\sigma_{\Delta \mathrm{keff}}$ & $\mathrm{N}$ \\
\hline \hline \multirow{2}{*}{1} & $-1 \mathrm{~cm}(10 \times$ limit $)$ & 0.00036 & \pm & 0.00016 & $10 \sqrt{ } 3$ & -0.00002 & \pm & 0.00001 & \\
& $+1 \mathrm{~cm}(10 \times$ limit $)$ & -0.00046 & \pm & 0.00016 & $10 \sqrt{ } 3$ & -0.00003 & \pm & 0.00001 & 22.5 \\
\hline \multirow{2}{*}{2} & $-1 \mathrm{~cm}(10 \times$ limit $)$ & 0.00188 & \pm & 0.00017 & $10 \sqrt{ } 3$ & -0.00011 & \pm & 0.00001 & \\
& $+1 \mathrm{~cm}(10 \times$ limit $)$ & -0.00192 & \pm & 0.00017 & $10 \sqrt{ } 3$ & -0.00011 & \pm & 0.00001 & 58.5 \\
\hline \multirow{2}{*}{3} & $-1 \mathrm{~cm}(10 \times$ limit $)$ & 0.00243 & \pm & 0.00017 & $10 \sqrt{3}$ & -0.00014 & \pm & 0.00001 & \\
& $+1 \mathrm{~cm}(10 \times$ limit $)$ & -0.00231 & \pm & 0.00017 & $10 \sqrt{3}$ & -0.00013 & \pm & 0.00001 & 78.0 \\
\hline \multirow{2}{*}{4} & $-1 \mathrm{~cm}(10 \times$ limit $)$ & 0.00136 & \pm & 0.00017 & $10 \sqrt{3}$ & -0.00008 & \pm & 0.00001 & \\
& $+1 \mathrm{~cm}(10 \times$ limit $)$ & -0.00130 & \pm & 0.00017 & $10 \sqrt{3}$ & -0.00008 & \pm & 0.00001 & 72.0 \\
\hline \multirow{2}{*}{5} & $-1 \mathrm{~cm}(10 \times$ limit $)$ & 0.00235 & \pm & 0.00016 & $10 \sqrt{3}$ & -0.00014 & \pm & 0.00001 & \\
& $+1 \mathrm{~cm}(10 \times$ limit $)$ & -0.00258 & \pm & 0.00016 & $10 \sqrt{3}$ & -0.00015 & \pm & 0.00001 & 91.0 \\
\hline \hline
\end{tabular}

Table 2.24. Effect of Uncertainty in CR Clad Dimensions (Spine Diameter).

\begin{tabular}{|c|c|c|c|c|c|c|c|c|c|}
\hline Case & Deviation & $\Delta \mathrm{k}$ & \pm & $\sigma_{\Delta \mathrm{k}}$ & $\begin{array}{c}\text { Scaling } \\
\text { Factor }\end{array}$ & $\Delta \mathrm{k}_{\mathrm{eff}}(1 \sigma)$ & \pm & $\sigma_{\Delta \mathrm{keff}}$ & $\mathrm{N}$ \\
\hline \multirow{2}{*}{1} & $-10 \mathrm{~mm}(20 \times$ limit $)$ & -0.00002 & \pm & 0.00017 & $20 \sqrt{3}$ & 0.00000 & \pm & 0.00000 & \multirow{2}{*}{22.5} \\
\hline & $+10 \mathrm{~mm}(20 \times$ limit $)$ & 0.00001 & \pm & 00017 & $20 \sqrt{3}$ & 0.00000 & \pm & 0.00000 & \\
\hline \multirow{2}{*}{2} & $-10 \mathrm{~mm}(20 \times$ limit $)$ & 0.00008 & \pm & 0.00017 & $20 \sqrt{3}$ & 0.00000 & \pm & 0.00000 & \multirow{2}{*}{58.5} \\
\hline & $+10 \mathrm{~mm}(20 \times$ limit $)$ & 0.00014 & \pm & 0.00016 & $20 \sqrt{3}$ & 000 & \pm & 000 & \\
\hline \multirow{2}{*}{3} & $-10 \mathrm{~mm}(20 \times$ limit $)$ & -0.00002 & \pm & 0.00017 & $20 \sqrt{3}$ & 0.00000 & \pm & 0.00000 & \multirow{2}{*}{78.0} \\
\hline & $+10 \mathrm{~mm}(20 \times$ limit $)$ & -0.00017 & 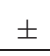 & 0.00017 & $20 \sqrt{3}$ & 0.00000 & \pm & 0.00000 & \\
\hline \multirow{2}{*}{4} & $-10 \mathrm{~mm}(20 \times$ limit $)$ & -0.00010 & \pm & 0.00017 & $20 \sqrt{3}$ & 0.00000 & \pm & 0.00000 & \multirow{2}{*}{72.0} \\
\hline & $+10 \mathrm{~mm}(20 \times$ limit $)$ & 0.00010 & 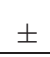 & 0.00016 & $20 \sqrt{3}$ & 0.00000 & $\perp$ & 0.00000 & \\
\hline & $-10 \mathrm{~mm}(20 \times$ limit $)$ & -0.00026 & \pm & 0.00016 & $20 \sqrt{3}$ & -0.00001 & \pm & 0.00000 & \multirow{2}{*}{91.0} \\
\hline & $+10 \mathrm{~mm}(20 \times$ limit $)$ & 0.00007 & \pm & 0.00016 & $20 \sqrt{3}$ & 0.00000 & \pm & 0.00000 & \\
\hline
\end{tabular}

\subsubsection{Instrumentation}

Insufficient information is available to comprehensively model and evaluate the uncertainties and biases related to the utility of instrumentation in the HTTR. Neglect of instrumentation inclusion in the model would be a bias; uncertainty in the dimensions and composition of the instrumentation would provide uncertainty in that bias or uncertainty in the model should it have been included in the benchmark model. An approximation of the instrumentation in the HTTR was modeled using information from Section 1.1.2.3 of HTTR-GCR-RESR-001 and approximate diagrams shown in a presentation at the IAEA CRP- 
NEA/NSC/DOC(2006)1

\section{Gas Cooled (Thermal) Reactor - GCR \\ HTTR-GCR-RESR-002 \\ CRIT-REAC-RRATE}

5 Meeting. ${ }^{a}$ The expected bias in the instrumentation, from the aforementioned reference, is $\sim 0.2 \%$ $\Delta \mathrm{k} / \mathrm{k}$.

Figures 2.1 through 2.4 provide basic geometric descriptions of the instrumentation utilized in the HTTR core. Figure 2.1 shows the respective heights. Figure 2.2, 2.3, and 2.4 provide additional information regarding channels 1, 2, and 3, respectively (columns E05, E13, and E21, respectively, in Figure 2.5). The 5-cm long $0.6-\mathrm{cm}$ diameter $\mathrm{BF}_{3}$ counters were modeled containing gas at 1 atm with 100 at. $\%{ }^{10} \mathrm{~B}$ content. All metallic components were modeled as aluminum. Approximate dimensions were used based on scaling of the Figures 2.2 through 2.4 and the known hole diameter of $123 \mathrm{~mm}$.

Approximate biases were calculated for the annular core configurations (Table 3.1). The bias for the fully-loaded core critical was calculated to be $0.254 \pm 0.073 \% \Delta \mathrm{k} / \mathrm{k}$ (HTTR-GCR-RESR-001 Section 3.1.1.1), which is similar to the previously reported value. The uncertainty in the biases was approximated by dividing the biases in half, and then treating it as a bounding uncertainty and dividing by $\sqrt{3}$. The uncertainty in the instrumentation is included in the total benchmark model uncertainty, and is provided in Table 3.1. The instrumentation is not included in the benchmark model but the bias in used to correct the experimental $\mathrm{k}_{\text {eff. }}$. Because the actual dimensions and material properties are approximated, this uncertainty is treated as completely systematic with no random components.

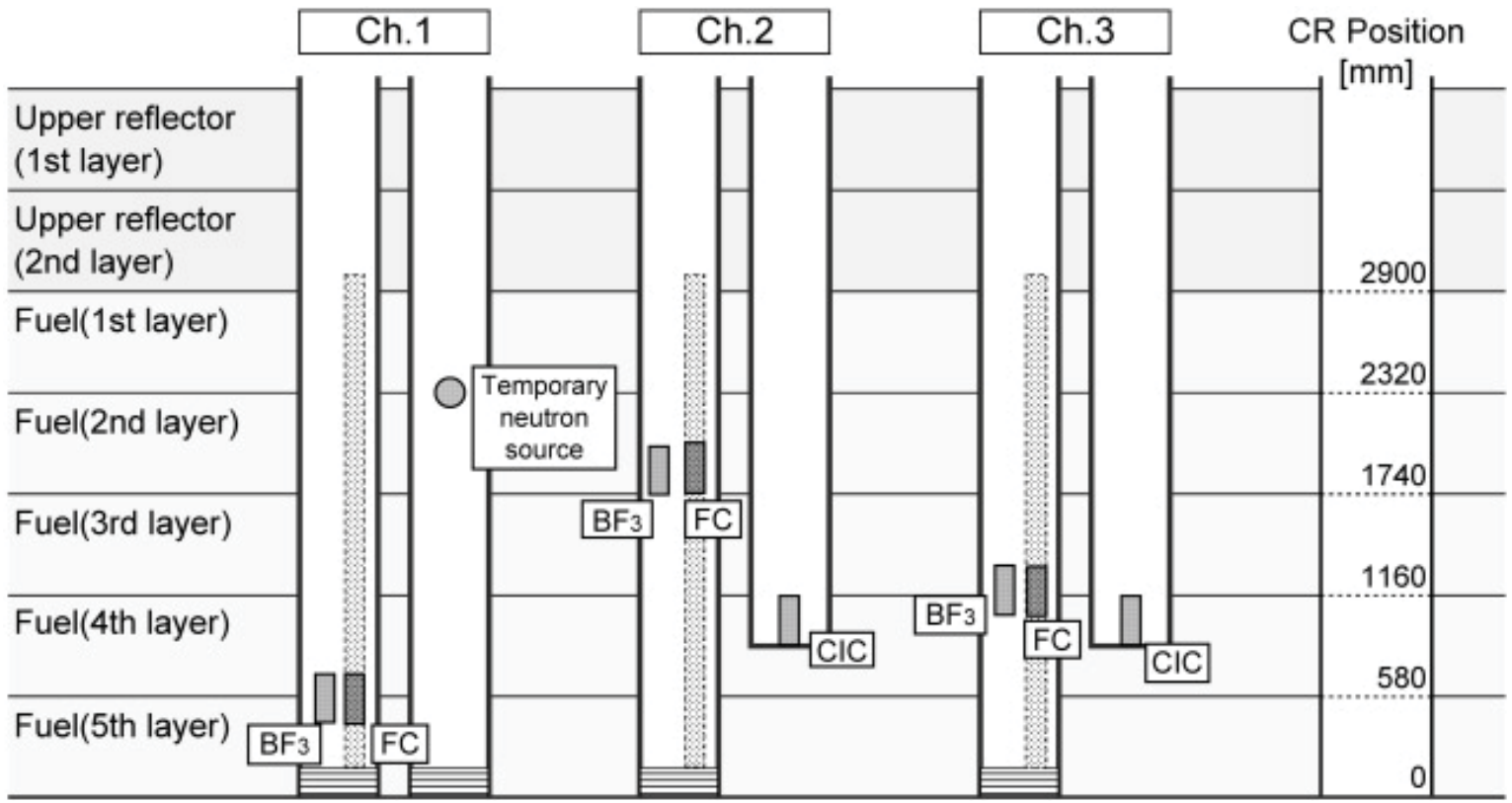

Figure 2.1. Vertical Position of the Temporary Neutron Detectors (Ref 1, p. 314).

$\mathrm{BF}_{3}=$ boron-trifluoride neutron detector

$\mathrm{CIC}=$ gamma-ray compensated ionization chamber

$\mathrm{FC}=$ micro-fission chamber

\footnotetext{
a N. Fujimoto, N. Nojiri, and K. Yamashita, "HTTR's Benchmark Calculation of Start-Up Core Physics Tests," Report of the $3^{\text {rd }}$ Research Coordiation Meeting on the CRP, IAEA, Oarai, Japan, March 12-16 (2001).
} 
NEA/NSC/DOC(2006)1

Gas Cooled (Thermal) Reactor - GCR

HTTR-GCR-RESR-002

CRIT-REAC-RRATE

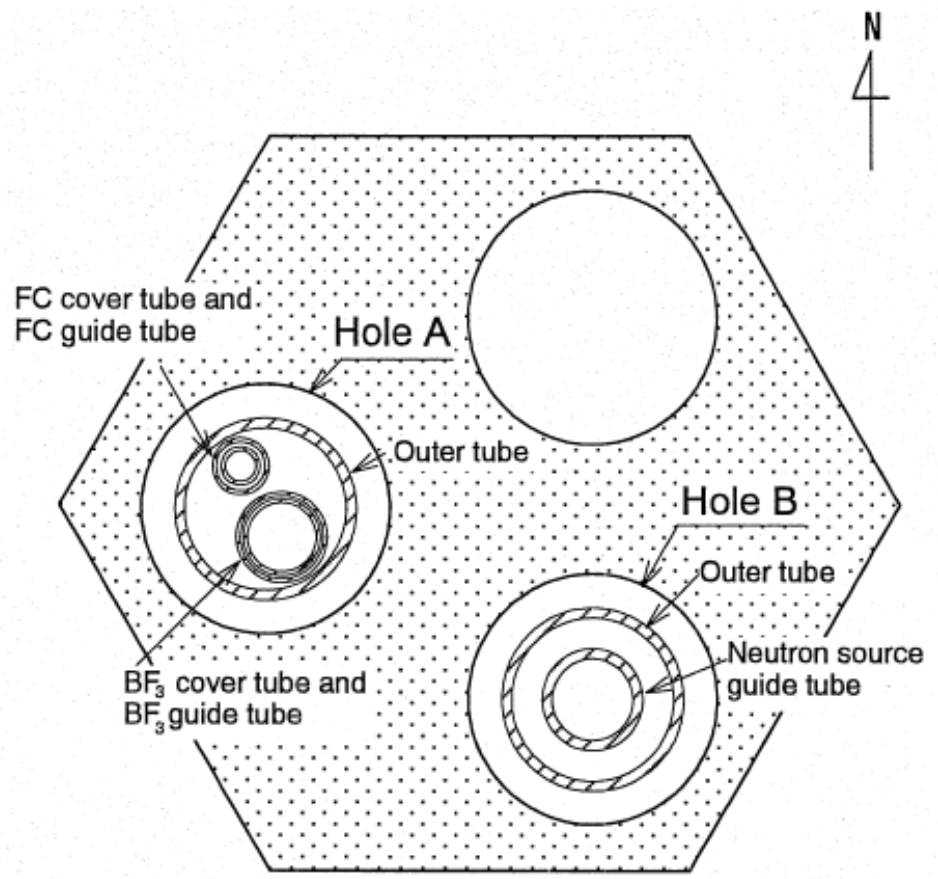

Horizontal cross section

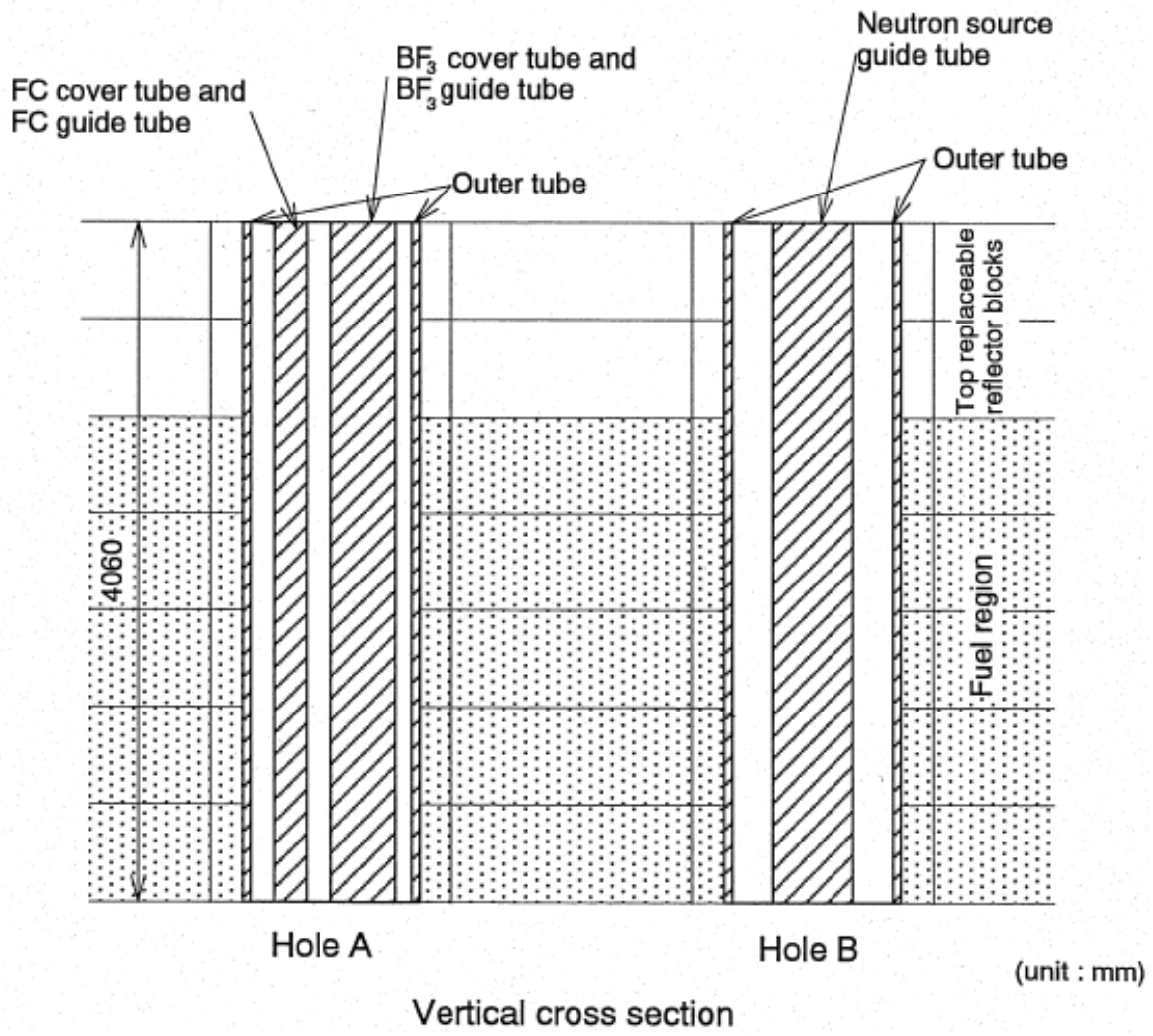

Figure 2.2. Approximation of Instrumentation Channel 1 (Column E05). ${ }^{\mathrm{a}}$

\footnotetext{
${ }^{a}$ N. Fujimoto, N. Nojiri, and K. Yamashita, "HTTR's Benchmark Calculation of Start-Up Core Physics Tests," Report of the $3^{\text {rd }}$ Research Coordination Meeting on the CRP, IAEA, Oarai, Japan, March 12-16 (2001).
} 
NEA/NSC/DOC(2006)1

Gas Cooled (Thermal) Reactor - GCR

HTTR-GCR-RESR-002

CRIT-REAC-RRATE

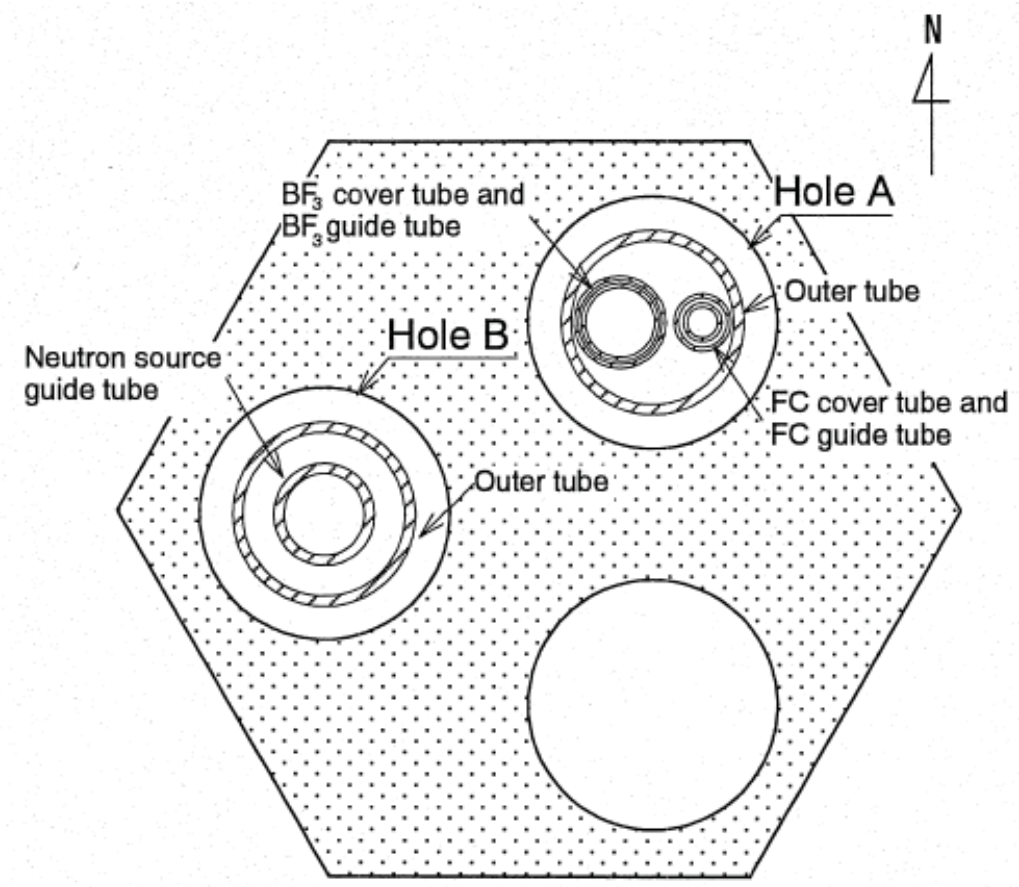

Horizontal cross section

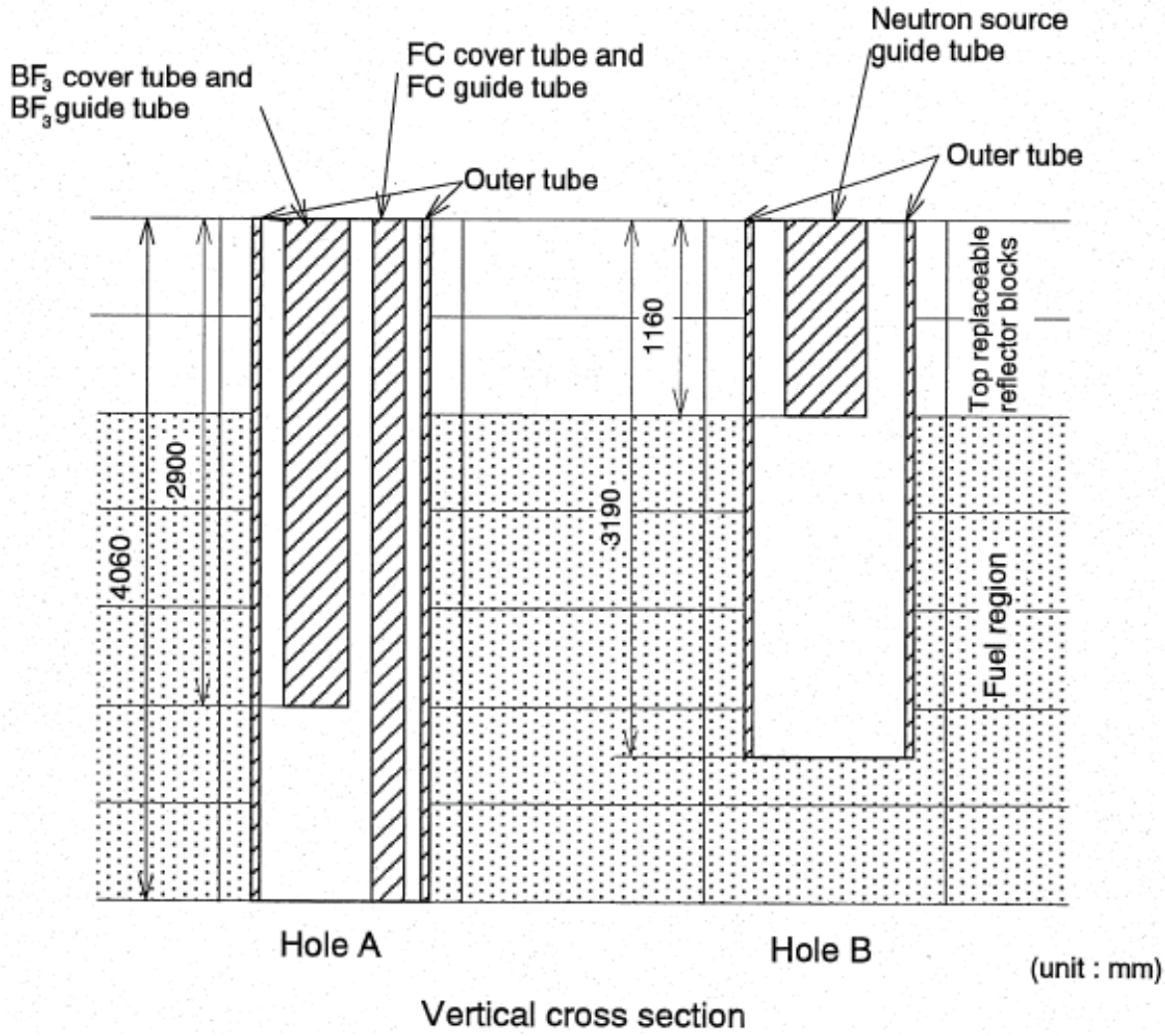

Figure 2.3. Approximation of Instrumentation Channel 2 (Column E13). ${ }^{a}$

\footnotetext{
${ }^{a}$ N. Fujimoto, N. Nojiri, and K. Yamashita, "HTTR's Benchmark Calculation of Start-Up Core Physics Tests," Report of the $3^{\text {rd }}$ Research Coordination Meeting on the CRP, IAEA, Oarai, Japan, March 12-16 (2001).
} 
NEA/NSC/DOC(2006)1

Gas Cooled (Thermal) Reactor - GCR

HTTR-GCR-RESR-002

CRIT-REAC-RRATE

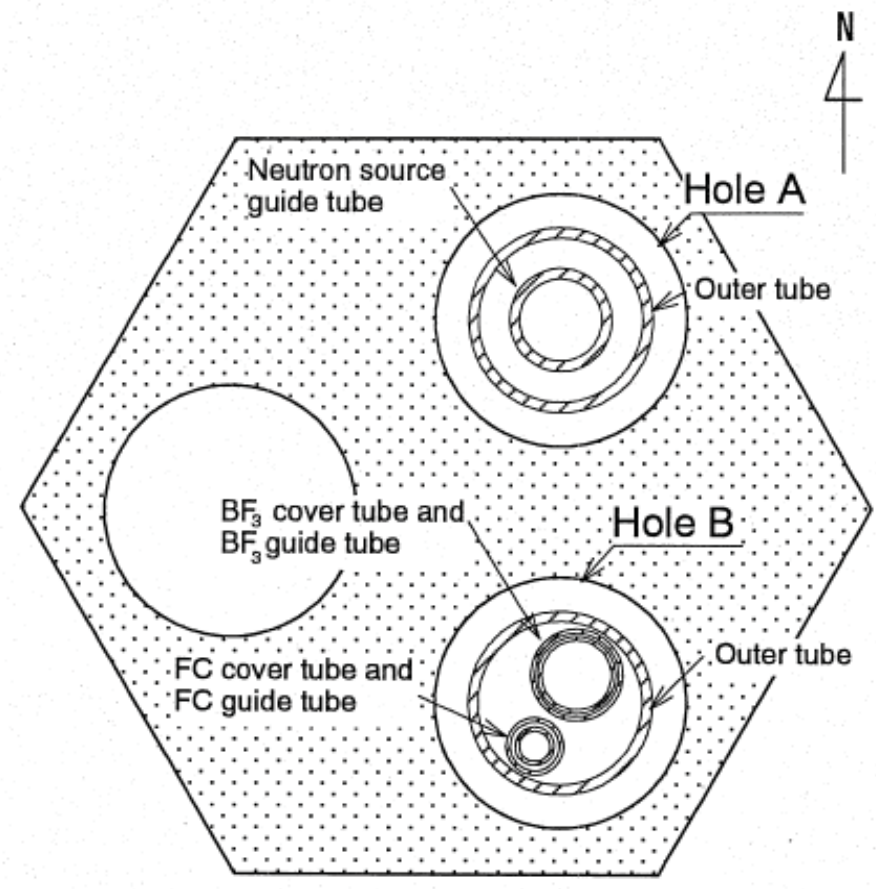

Horizontal cross section

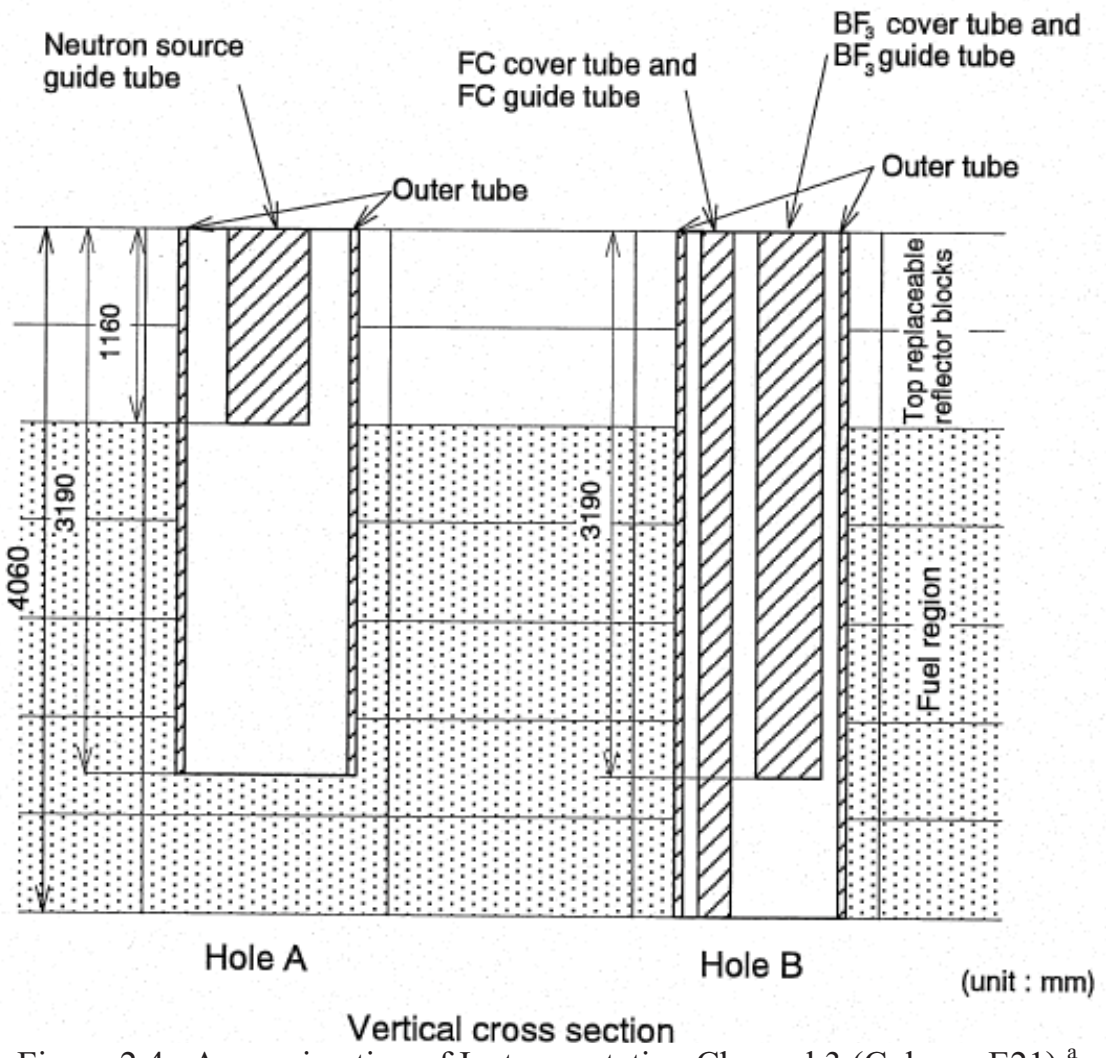

Figure 2.4. Approximation of Instrumentation Channel 3 (Column E21).

\footnotetext{
${ }^{a}$ N. Fujimoto, N. Nojiri, and K. Yamashita, "HTTR's Benchmark Calculation of Start-Up Core Physics Tests," Report of the $3^{\text {rd }}$ Research Coordination Meeting on the CRP, IAEA, Oarai, Japan, March 12-16 (2001).
} 
NEA/NSC/DOC(2006)1

Gas Cooled (Thermal) Reactor - GCR

HTTR-GCR-RESR-002

CRIT-REAC-RRATE

North
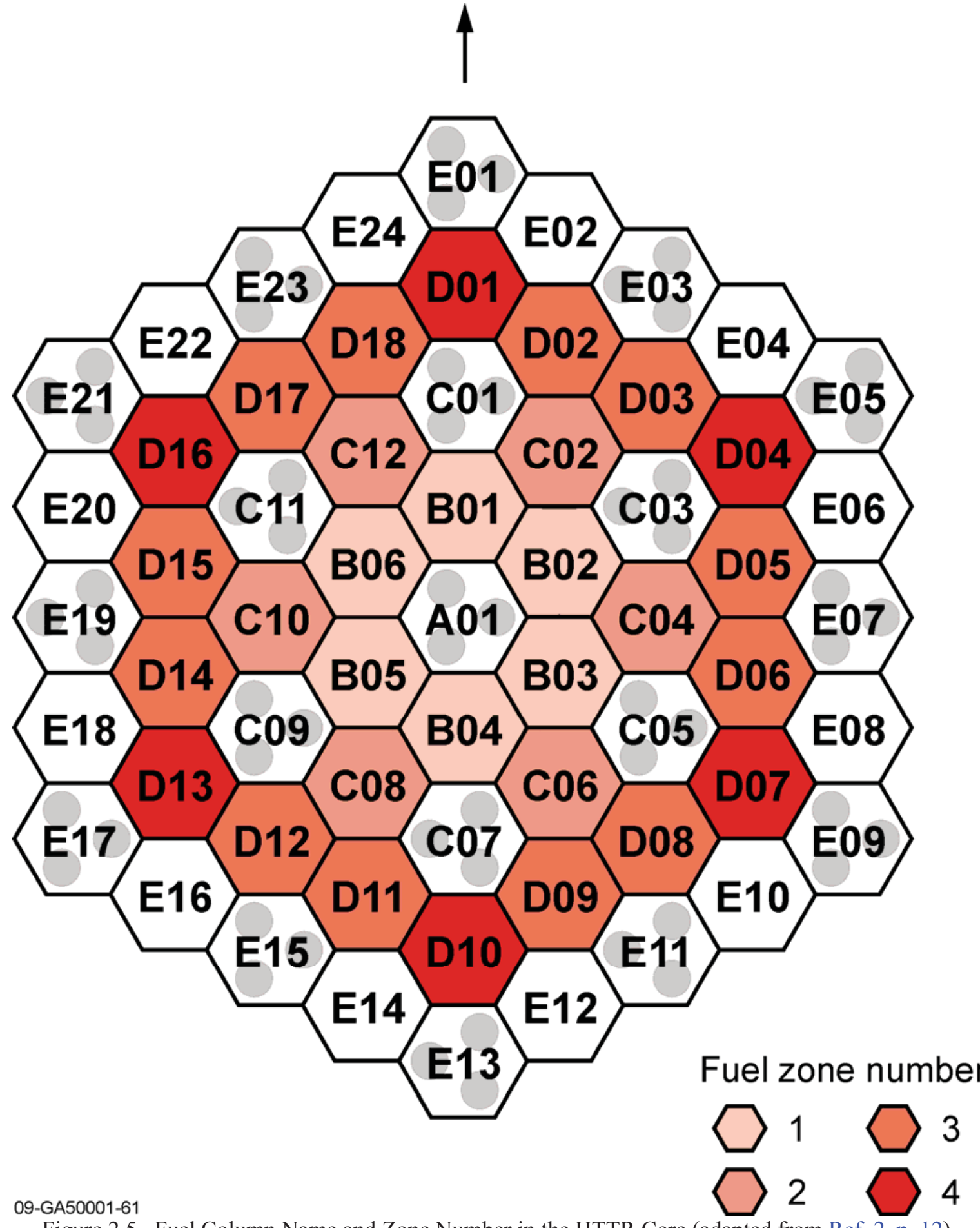

Figure 2.5. Fuel Column Name and Zone Number in the HTTR Core (adapted from Ref. 2, p. 12). 
NEA/NSC/DOC(2006)1

Gas Cooled (Thermal) Reactor - GCR

HTTR-GCR-RESR-002

CRIT-REAC-RRATE

\subsubsection{Graphite Blocks}

\section{Block Dimensions}

An uncertainty in the block dimensions was unreported. The uncertainty in the flat-to-flat distance and the height were each varied $\pm 1 \mathrm{~mm}$ from their nominal values of 360 and $580 \mathrm{~cm}$ (Tables 1.13 and 1.16 of HTTR-GCR-RESR-001), respectively, and their effects on the uncertainty of $\mathrm{k}_{\text {eff }}$ were determined. The average gap between columns was reported as approximately $2 \mathrm{~mm}$ (Ref. 2, p. 13), allowing room for the flat-to-flat uncertainty. All graphite blocks were varied with this analysis: fuel, reflector, control, instrumentation, and dummy. Results are shown in Tables 2.25 and 2.26.

Because of the tight manufacturing tolerances of the fuel compacts and graphite sleeves (Sections 2.1.2.2 and 2.1.2.3, respectively) it is believed that similar tolerances apply to other graphite and boron carbide components of the HTTR. Therefore, the graphite block dimensions are treated with a tolerance (with uniform probability) of $\pm 0.1 \mathrm{~mm}$. The appropriate corrections to the scaling factors have been incorporated into the uncertainty analysis of these parameters.

The total number of graphite blocks used in all core configurations is 549. For determining the random component of the uncertainty, the results in Tables 2.25 and 2.26 would be divided by $\sqrt{549}$.

Table 2.25. Effect of Uncertainty in Graphite Block Dimensions (Flat-to-Flat Distance).

\begin{tabular}{||c|c|ccc|c|ccc||}
\hline Case & Deviation & $\Delta \mathrm{k}$ & \pm & $\sigma_{\Delta \mathrm{k}}$ & $\begin{array}{c}\text { Scaling } \\
\text { Factor }\end{array}$ & $\Delta \mathrm{k}_{\text {eff }}(1 \sigma)$ & \pm & $\sigma_{\Delta \mathrm{keff}}$ \\
\hline \hline \multirow{2}{*}{1} & $-1 \mathrm{~mm}(10 \times$ limit $)$ & -0.00263 & \pm & 0.00016 & $10 \sqrt{3}$ & -0.00015 & \pm & 0.00001 \\
& $+1 \mathrm{~mm}(10 \times$ limit $)$ & 0.00238 & \pm & 0.00017 & $10 \sqrt{ } 3$ & 0.00014 & \pm & 0.00001 \\
\hline \multirow{2}{*}{2} & $-1 \mathrm{~mm}(10 \times$ limit $)$ & -0.00242 & \pm & 0.00016 & $10 \sqrt{3}$ & -0.00014 & \pm & 0.00001 \\
& $+1 \mathrm{~mm}(10 \times$ limit $)$ & 0.00222 & \pm & 0.00017 & $10 \sqrt{3}$ & 0.00013 & \pm & 0.00001 \\
\hline \multirow{3}{*}{3} & $-1 \mathrm{~mm}(10 \times$ limit $)$ & -0.00335 & \pm & 0.00017 & $10 \sqrt{3}$ & -0.00019 & \pm & 0.00001 \\
& $+1 \mathrm{~mm}(10 \times$ limit $)$ & 0.00247 & \pm & 0.00016 & $10 \sqrt{3}$ & 0.00014 & \pm & 0.00001 \\
\hline \multirow{3}{*}{4} & $-1 \mathrm{~mm}(10 \times$ limit $)$ & -0.00385 & \pm & 0.00017 & $10 \sqrt{3}$ & -0.00022 & \pm & 0.00001 \\
& $+1 \mathrm{~mm}(10 \times$ limit $)$ & 0.00308 & \pm & 0.00017 & $10 \sqrt{3}$ & 0.00018 & \pm & 0.00001 \\
\hline \multirow{2}{*}{5} & $-1 \mathrm{~mm}(10 \times$ limit $)$ & -0.00384 & \pm & 0.00016 & $10 \sqrt{3}$ & -0.00022 & \pm & 0.00001 \\
& $+1 \mathrm{~mm}(10 \times$ limit $)$ & 0.00311 & \pm & 0.00016 & $10 \sqrt{3}$ & 0.00018 & \pm & 0.00001 \\
\hline \hline
\end{tabular}


NEA/NSC/DOC(2006)1

Gas Cooled (Thermal) Reactor - GCR

HTTR-GCR-RESR-002

CRIT-REAC-RRATE

Table 2.26. Effect of Uncertainty in Graphite Block Dimensions (Height).

\begin{tabular}{||c|c|ccc|c|ccc||}
\hline Case & Deviation & $\Delta \mathrm{k}$ & \pm & $\sigma_{\Delta \mathrm{k}}$ & $\begin{array}{c}\text { Scaling } \\
\text { Factor }\end{array}$ & $\Delta \mathrm{k}_{\text {eff }}(1 \sigma)$ & \pm & $\sigma_{\Delta \mathrm{keff}}$ \\
\hline \hline \multirow{2}{*}{1} & $-1 \mathrm{~mm}(10 \times$ limit $)$ & 0.00006 & \pm & 0.00017 & $10 \sqrt{3}$ & 0.00000 & \pm & 0.00001 \\
& $+1 \mathrm{~mm}(10 \times$ limit $)$ & -0.00004 & \pm & 0.00016 & $10 \sqrt{3}$ & 0.00000 & \pm & 0.00001 \\
\hline \multirow{2}{*}{2} & $-1 \mathrm{~mm}(10 \times$ limit $)$ & 0.00005 & \pm & 0.00017 & $10 \sqrt{3}$ & 0.00000 & \pm & 0.00001 \\
& $+1 \mathrm{~mm}(10 \times$ limit $)$ & 0.00010 & \pm & 0.00017 & $10 \sqrt{3}$ & 0.00001 & \pm & 0.00001 \\
\hline \multirow{2}{*}{3} & $-1 \mathrm{~mm}(10 \times$ limit $)$ & 0.00006 & \pm & 0.00017 & $10 \sqrt{3}$ & 0.00000 & \pm & 0.00001 \\
& $+1 \mathrm{~mm}(10 \times$ limit $)$ & 0.00001 & \pm & 0.00017 & $10 \sqrt{3}$ & 0.00000 & \pm & 0.00001 \\
\hline & $-1 \mathrm{~mm}(10 \times$ limit $)$ & -0.00010 & \pm & 0.00017 & $10 \sqrt{3}$ & -0.00001 & \pm & 0.00001 \\
& $+1 \mathrm{~mm}(10 \times$ limit $)$ & -0.00015 & \pm & 0.00017 & $10 \sqrt{3}$ & -0.00001 & \pm & 0.00001 \\
\hline \multirow{2}{*}{5} & $-1 \mathrm{~mm}(10 \times$ limit $)$ & -0.00002 & \pm & 0.00016 & $10 \sqrt{3}$ & 0.00000 & \pm & 0.00001 \\
& $+1 \mathrm{~mm}(10 \times$ limit $)$ & -0.00006 & \pm & 0.00016 & $10 \sqrt{3}$ & 0.00000 & \pm & 0.00001 \\
\hline
\end{tabular}

\section{Dowel/Socket Dimensions}

Insufficient information is available to completely model and evaluate the uncertainties and biases related to the incorporation of dowels and sockets in the HTTR. Uncertainty in the volume fraction will be included as part of the assessment of the uncertainty in the total density of the graphite blocks.

\section{Coolant Channel Diameter}

An assumed variation of $\pm 2 \mathrm{~mm}$ in the diameter of the coolant channels of the fuel blocks (nominally 41 $\mathrm{mm}$, Table 1.13 of HTTR-GCR-RESR-001) and reflector blocks (nominally $23 \mathrm{~mm}$ ) in the fuel columns was performed to determine the effective uncertainty in $\mathrm{k}_{\text {eff. }}$ Results are shown in Tables 2.27 and 2.28.

Insufficient information was available to determine the dimensions of the coolant channels of the lowest reflector blocks. They were modeled similar to the other reflector blocks utilized in the fuel columns. No bias or biased uncertainty was assessed.

Because of the tight manufacturing tolerances of the fuel compacts and graphite sleeves (Sections 2.1.2.2 and 2.1.2.3, respectively) it is believed that similar tolerances apply to other graphite and boron carbide components of the HTTR. Therefore, the coolant channel diameters are treated with a tolerance (with uniform probability) of $\pm 0.1 \mathrm{~mm}$. The appropriate corrections to the scaling factors have been incorporated into the uncertainty analysis of these parameters.

The total number of fuel coolant channels used in the fully-loaded core is 4,770. For determining the random component of the uncertainty, the results in Table 2.27 would be divided by $\sqrt{ } \mathrm{N}$. The total number of reflector coolant channels in all core configurations is 3,816. For determining the random component of the uncertainty, the results in Table 2.28 would be divided by $\sqrt{3}, 816$. 
NEA/NSC/DOC(2006)1

Gas Cooled (Thermal) Reactor - GCR

HTTR-GCR-RESR-002

CRIT-REAC-RRATE

Table 2.27. Effect of Uncertainty in Coolant Channel Diameter (Fuel Blocks).

\begin{tabular}{|c|c|c|c|c|c|c|c|c|c|}
\hline Case & Deviation & $\Delta \mathrm{k}$ & \pm & $\sigma_{\Delta \mathrm{k}}$ & $\begin{array}{l}\text { Scaling } \\
\text { Factor }\end{array}$ & $\Delta \mathrm{k}_{\mathrm{eff}}(1 \sigma)$ & \pm & $\sigma_{\Delta \mathrm{keff}}$ & $\mathrm{N}$ \\
\hline \multirow{2}{*}{1} & $-2 \mathrm{~mm}(20 \times$ limit $)$ & 0.00620 & & 0.00017 & $20 \sqrt{3}$ & 0.00018 & \pm & 0.00000 & \multirow{2}{*}{2,955} \\
\hline & $+2 \mathrm{~mm}(20 \times$ limit $)$ & -0.00644 & & 0.00017 & $20 \sqrt{3}$ & -0.00019 & \pm & 0.00000 & \\
\hline \multirow{2}{*}{2} & $-2 \mathrm{~mm}(20 \times$ limit $)$ & 0.00694 & \pm & 0.00016 & $20 \sqrt{3}$ & 0.00020 & \pm & 0.00000 & \multirow{2}{*}{3,285} \\
\hline & $+2 \mathrm{~mm}(20 \times$ limit $)$ & -0.00705 & 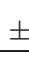 & 00017 & $\sqrt{3}$ & -0.00020 & \pm & 0000 & \\
\hline \multirow{2}{*}{3} & $-2 \mathrm{~mm}(20 \times$ limit $)$ & 0.00730 & \pm & 0.00016 & $20 \sqrt{3}$ & 0.00021 & \pm & 0.00000 & \multirow{2}{*}{3,780} \\
\hline & $+2 \mathrm{~mm}(20 \times$ limit $)$ & -0.00814 & 5 & 0.00016 & $20 \sqrt{3}$ & -0.00023 & \pm & 0.00000 & \\
\hline \multirow{2}{*}{4} & $-2 \mathrm{~mm}(20 \times$ limit $)$ & 0.00837 & \pm & 0.00016 & $20 \sqrt{3}$ & 0.00024 & \pm & 0.00000 & \multirow{2}{*}{3,780} \\
\hline & $+2 \mathrm{~mm}(20 \times$ limit $)$ & -0.00929 & 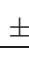 & 0.00017 & $20 \sqrt{3}$ & -0.00027 & \pm & 0.00000 & \\
\hline & $-2 \mathrm{~mm}(20 \times$ limit $)$ & 0.00787 & & 0.00016 & $20 \sqrt{3}$ & 0.00023 & \pm & 0.00000 & \multirow{2}{*}{4,275} \\
\hline & $+2 \mathrm{~mm}(20 \times$ limit $)$ & -0.00889 & \pm & 0.00016 & $20 \sqrt{3}$ & -0.00026 & \pm & 0.00000 & \\
\hline
\end{tabular}

Table 2.28. Effect of Uncertainty in Coolant Channel Diameter (Reflector Blocks).

\begin{tabular}{||c|c|ccc|c|ccc||}
\hline Case & Deviation & $\Delta \mathrm{k}$ & \pm & $\sigma_{\Delta \mathrm{k}}$ & $\begin{array}{c}\text { Scaling } \\
\text { Factor }\end{array}$ & $\Delta \mathrm{k}_{\text {eff }}(1 \sigma)$ & \pm & $\sigma_{\Delta \mathrm{keff}}$ \\
\hline \hline \multirow{2}{*}{1} & $-2 \mathrm{~mm}(20 \times$ limit $)$ & 0.00039 & \pm & 0.00016 & $20 \sqrt{ } 3$ & 0.00001 & \pm & 0.00000 \\
& $+2 \mathrm{~mm}(20 \times$ limit $)$ & -0.00072 & \pm & 0.00017 & $20 \sqrt{ } 3$ & -0.00002 & \pm & 0.00000 \\
\hline \multirow{2}{*}{2} & $-2 \mathrm{~mm}(20 \times$ limit $)$ & 0.00045 & \pm & 0.00017 & $20 \sqrt{ } 3$ & 0.00001 & \pm & 0.00000 \\
& $+2 \mathrm{~mm}(20 \times$ limit $)$ & -0.00008 & \pm & 0.00016 & $20 \sqrt{3}$ & 0.00000 & \pm & 0.00000 \\
\hline \multirow{3}{*}{3} & $-2 \mathrm{~mm}(20 \times$ limit $)$ & 0.00028 & \pm & 0.00016 & $20 \sqrt{3}$ & 0.00001 & \pm & 0.00000 \\
& $+2 \mathrm{~mm}(20 \times$ limit $)$ & -0.00072 & \pm & 0.00016 & $20 \sqrt{3}$ & -0.00002 & \pm & 0.00000 \\
\hline \multirow{3}{*}{4} & $-2 \mathrm{~mm}(20 \times$ limit $)$ & 0.00009 & \pm & 0.00017 & $20 \sqrt{3}$ & 0.00000 & \pm & 0.00000 \\
& $+2 \mathrm{~mm}(20 \times$ limit $)$ & -0.00054 & \pm & 0.00017 & $20 \sqrt{3}$ & -0.00002 & \pm & 0.00000 \\
\hline \multirow{2}{*}{5} & $-2 \mathrm{~mm}(20 \times$ limit $)$ & 0.00026 & \pm & 0.00016 & $20 \sqrt{3}$ & 0.00001 & \pm & 0.00000 \\
& $+2 \mathrm{~mm}(20 \times$ limit $)$ & -0.00045 & \pm & 0.00016 & $20 \sqrt{3}$ & -0.00001 & \pm & 0.00000 \\
\hline \hline
\end{tabular}

\section{Fuel and Coolant Channel Pitch}

An uncertainty in the fuel and coolant channel pitches in the fuel columns was not reported. For the evaluation of this uncertainty, the channels were modeled closer together then further apart by adjusting the pitch between them. A variation of $\pm 2.0 \mathrm{~mm}$ from the nominal pitch of $51.5 \mathrm{~mm}$ (Figures 1.52 and 1.53 of HTTR-GCR-RESR-001) was assumed and the effects on the uncertainty of $\mathrm{k}_{\mathrm{eff}}$ were determined. Results are shown in Table 2.29.

Because of the tight manufacturing tolerances of the fuel compacts and graphite sleeves (Sections 2.1.2.2 and 2.1.2.3, respectively) it is believed that similar tolerances apply to other graphite and boron carbide components of the HTTR. Therefore, the coolant channel pitch is treated with a tolerance (with uniform 
NEA/NSC/DOC(2006)1

\section{Gas Cooled (Thermal) Reactor - GCR \\ HTTR-GCR-RESR-002 \\ CRIT-REAC-RRATE}

probability) of $\pm 0.1 \mathrm{~mm}$. The appropriate corrections to the scaling factors have been incorporated into the uncertainty analysis of these parameters.

The total number of pitch positions used in the fully-loaded core is 8,586 . For determining the random component of the uncertainty, the results in Table 2.29 would be divided by $\sqrt{ } \mathrm{N}$, where $\mathrm{N}$ for each case is shown in Table 2.29.

Table 2.29. Effect of Uncertainty in Fuel and Coolant Channel Pitch.

\begin{tabular}{||c|c|ccc|c|ccc|c||}
\hline \hline Case & Deviation & $\Delta \mathrm{k}$ & \pm & $\sigma_{\Delta \mathrm{k}}$ & $\begin{array}{c}\text { Scaling } \\
\text { Factor }\end{array}$ & $\Delta \mathrm{k}_{\text {eff }}(1 \sigma)$ & \pm & $\sigma_{\Delta \mathrm{keff}}$ & $\mathrm{N}$ \\
\hline \hline \multirow{2}{*}{1} & $-2 \mathrm{~mm}(20 \times$ limit $)$ & -0.00620 & \pm & 0.00017 & $20 \sqrt{3}$ & -0.00018 & \pm & 0.00000 & \\
& $+2 \mathrm{~mm}(20 \times$ limit $)$ & 0.00628 & \pm & 0.00017 & $20 \sqrt{3}$ & 0.00018 & \pm & 0.00000 & 6,771 \\
\hline \multirow{2}{*}{2} & $-2 \mathrm{~mm}(20 \times$ limit $)$ & -0.00584 & \pm & 0.00016 & $20 \sqrt{3}$ & -0.00017 & \pm & 0.00000 & \\
& $+2 \mathrm{~mm}(20 \times$ limit $)$ & 0.00583 & \pm & 0.00016 & $20 \sqrt{3}$ & 0.00017 & \pm & 0.00000 & 7,101 \\
\hline \multirow{2}{*}{3} & $-2 \mathrm{~mm}(20 \times$ limit $)$ & -0.00510 & \pm & 0.00016 & $20 \sqrt{3}$ & -0.00015 & \pm & 0.00000 & \\
& $+2 \mathrm{~mm}(20 \times$ limit $)$ & 0.00472 & \pm & 0.00017 & $20 \sqrt{3}$ & 0.00014 & \pm & 0.00000 & 7,596 \\
\hline \multirow{2}{*}{4} & $-2 \mathrm{~mm}(20 \times$ limit $)$ & -0.00485 & \pm & 0.00017 & $20 \sqrt{3}$ & -0.00014 & \pm & 0.00000 & \\
& $+2 \mathrm{~mm}(20 \times$ limit $)$ & 0.00474 & \pm & 0.00017 & $20 \sqrt{3}$ & 0.00014 & \pm & 0.00000 & 7,596 \\
\hline \multirow{2}{*}{5} & $-2 \mathrm{~mm}(20 \times$ limit $)$ & -0.00413 & \pm & 0.0016 & $20 \sqrt{3}$ & -0.00012 & \pm & 0.00000 & \\
& $+2 \mathrm{~mm}(20 \times$ limit $)$ & 0.00411 & \pm & 0.00016 & $20 \sqrt{3}$ & 0.00012 & \pm & 0.00000 & 8,091 \\
\hline
\end{tabular}

\section{Handling Socket Dimensions}

The handling sockets were not included in the model as there was insufficient information to model them completely. The calculated volume of the socket (estimated using dimensions in Figure 1.52 of HTTR-GCR-RESR-001) is roughly 0.5 vol. $\%$ of the complete block envelope. This volume reduction is included as a reduction in total block density in the benchmark model. A bias has not been assessed. Uncertainty in the volume fraction will be included as part of the assessment of the uncertainty in the total density of the graphite blocks.

\section{Column Pitch}

An uncertainty in column pitch was assumed based upon the average distance between blocks of approximately $2 \mathrm{~mm}$. For the evaluation of this uncertainty, the columns were modeled closer together then further apart by adjusting the pitch between them. A variation of $\pm 2 \mathrm{~mm}(2 \times$ bounding limit) from the nominal value of $362 \mathrm{~mm}$ was analyzed and the effects on the uncertainty of $k_{\text {eff }}$ were determined. Results are shown in Table 2.30.

The total number of columns used in all core configurations is 61 . For determining the random component of the uncertainty, the results in Table 2.30 would be divided by $\sqrt{ } 61$. 
NEA/NSC/DOC(2006)1

Gas Cooled (Thermal) Reactor - GCR

HTTR-GCR-RESR-002

CRIT-REAC-RRATE

Table 2.30. Effect of Uncertainty in Column Pitch.

\begin{tabular}{||c|c|ccc|c|ccc||}
\hline Case & Deviation & $\Delta \mathrm{k}$ & \pm & $\sigma_{\Delta \mathrm{k}}$ & $\begin{array}{c}\text { Scaling } \\
\text { Factor }\end{array}$ & $\Delta \mathrm{k}_{\text {eff }}(1 \sigma)$ & \pm & $\sigma_{\Delta \mathrm{keff}}$ \\
\hline \multirow{2}{*}{1} & $-2 \mathrm{~mm}$ & 0.00204 & \pm & 0.00017 & $2 \sqrt{ } 3$ & 0.00059 & \pm & 0.00005 \\
& $+2 \mathrm{~mm}$ & -0.00249 & \pm & 0.00017 & $2 \sqrt{ } 3$ & -0.00072 & \pm & 0.00005 \\
\hline \multirow{2}{*}{2} & $-2 \mathrm{~mm}$ & 0.00215 & \pm & 0.00017 & $2 \sqrt{ } 3$ & 0.00062 & \pm & 0.00005 \\
& $+2 \mathrm{~mm}$ & -0.00231 & \pm & 0.00017 & $2 \sqrt{ } 3$ & -0.00067 & \pm & 0.00005 \\
\hline \multirow{3}{*}{3} & $-2 \mathrm{~mm}$ & 0.00203 & \pm & 0.00017 & $2 \sqrt{ } 3$ & 0.00059 & \pm & 0.00005 \\
& $+2 \mathrm{~mm}$ & -0.00251 & \pm & 0.00017 & $2 \sqrt{3}$ & -0.00072 & \pm & 0.00005 \\
\hline \multirow{3}{*}{4} & $-2 \mathrm{~mm}$ & 0.00185 & \pm & 0.00017 & $2 \sqrt{ } 3$ & 0.00053 & \pm & 0.00005 \\
& $+2 \mathrm{~mm}$ & -0.00267 & \pm & 0.00017 & $2 \sqrt{ } 3$ & -0.00077 & \pm & 0.00005 \\
\hline \multirow{2}{*}{5} & $-2 \mathrm{~mm}$ & 0.00219 & \pm & 0.00016 & $2 \sqrt{3}$ & 0.00063 & \pm & 0.00005 \\
& $+2 \mathrm{~mm}$ & -0.00224 & \pm & 0.00016 & $2 \sqrt{ } 3$ & -0.00065 & \pm & 0.00004 \\
\hline
\end{tabular}

\section{Control-Rod Channel Diameter}

An assumed variation of $\pm 2 \mathrm{~mm}$ in the diameter of the control-rod coolant channels (nominal value of $123 \mathrm{~mm}$, Figure 1.64 of HTTR-GCR-RESR-001) in the control block columns was performed to determine the effective uncertainty in $\mathrm{k}_{\text {eff. }}$ Results are shown in Table 2.31.

Because of the tight manufacturing tolerances of the fuel compacts and graphite sleeves (Sections 2.1.2.2 and 2.1.2.3, respectively) it is believed that similar tolerances apply to other graphite and boron carbide components of the HTTR. Therefore, the control-rod channel diameter is treated with a tolerance (with uniform probability) of $\pm 0.1 \mathrm{~mm}$. The appropriate corrections to the scaling factors have been incorporated into the uncertainty analysis of these parameters.

The total number of control-rod channels (including empty instrumentation channels) in blocks used in all core configurations is approximately 437 . For determining the random component of the uncertainty, the results in Table 2.31 would be divided by $\sqrt{ } 437$. 
NEA/NSC/DOC(2006)1

Gas Cooled (Thermal) Reactor - GCR

HTTR-GCR-RESR-002

CRIT-REAC-RRATE

Table 2.31. Effect of Uncertainty in CR Channel Diameter.

\begin{tabular}{||c|c|ccc|c|ccc||}
\hline Case & Deviation & $\Delta \mathrm{k}$ & \pm & $\sigma_{\Delta \mathrm{k}}$ & $\begin{array}{c}\text { Scaling } \\
\text { Factor }\end{array}$ & $\Delta \mathrm{k}_{\text {eff }}(1 \sigma)$ & \pm & $\sigma_{\Delta \mathrm{keff}}$ \\
\hline \hline \multirow{2}{*}{1} & $-2 \mathrm{~mm}(20 \times$ limit $)$ & 0.00111 & \pm & 0.00017 & $20 \sqrt{ } 3$ & 0.00003 & \pm & 0.00000 \\
& $+2 \mathrm{~mm}(20 \times$ limit $)$ & -0.00142 & \pm & 0.00017 & $20 \sqrt{ } 3$ & -0.00004 & \pm & 0.00000 \\
\hline \multirow{2}{*}{2} & $-2 \mathrm{~mm}(20 \times$ limit $)$ & 0.00136 & \pm & 0.00017 & $20 \sqrt{ } 3$ & 0.00004 & \pm & 0.00000 \\
& $+2 \mathrm{~mm}(20 \times$ limit $)$ & -0.00126 & \pm & 0.00016 & $20 \sqrt{3}$ & -0.00004 & \pm & 0.00000 \\
\hline \multirow{2}{*}{3} & $-2 \mathrm{~mm}(20 \times$ limit $)$ & 0.00131 & \pm & 0.00016 & $20 \sqrt{3}$ & 0.00004 & \pm & 0.00000 \\
& $+2 \mathrm{~mm}(20 \times$ limit $)$ & -0.00148 & \pm & 0.00016 & $20 \sqrt{3}$ & -0.00004 & \pm & 0.00000 \\
\hline \multirow{3}{*}{4} & $-2 \mathrm{~mm}(20 \times$ limit $)$ & 0.00122 & \pm & 0.00017 & $20 \sqrt{3}$ & 0.00004 & \pm & 0.00000 \\
& $+2 \mathrm{~mm}(20 \times$ limit $)$ & -0.00169 & \pm & 0.00017 & $20 \sqrt{3}$ & -0.00005 & \pm & 0.00000 \\
\hline \multirow{2}{*}{5} & $-2 \mathrm{~mm}(20 \times$ limit $)$ & 0.00144 & \pm & 0.00016 & $20 \sqrt{3}$ & 0.00004 & \pm & 0.00000 \\
& $+2 \mathrm{~mm}(20 \times$ limit $)$ & -0.00149 & \pm & 0.00016 & $20 \sqrt{3}$ & -0.00004 & \pm & 0.00000 \\
\hline
\end{tabular}

\section{Control-Rod Channel Pitch}

An uncertainty in control-rod coolant channel pitch in the fuel and reflector blocks was not reported. For the evaluation of this uncertainty, the channels were modeled closer together then further apart by adjusting the pitch between them. A variation of $\pm 2 \mathrm{~mm}$ from the nominal distance of $108 \mathrm{~mm}$ from the block axis (Figure 1.64 of HTTR-GCR-RESR-001) was assumed and the effects on the uncertainty of $\mathrm{k}_{\text {eff }}$ were determined. Results are shown in Table 2.32.

Because of the tight manufacturing tolerances of the fuel compacts and graphite sleeves (Sections 2.1.2.2 and 2.1.2.3, respectively) it is believed that similar tolerances apply to other graphite and boron carbide components of the HTTR. Therefore, the control-rod channel pitch is treated with a tolerance (with uniform probability) of $\pm 0.1 \mathrm{~mm}$. The appropriate corrections to the scaling factors have been incorporated into the uncertainty analysis of these parameters.

The total number of control-rod channels (including empty instrumentation channels) in blocks used in the all core configurations is approximately 437 . For determining the random component of the uncertainty, the results in Table 2.32 would be divided by $\sqrt{ } 437$. 
NEA/NSC/DOC(2006)1

Gas Cooled (Thermal) Reactor - GCR

HTTR-GCR-RESR-002

CRIT-REAC-RRATE

Table 2.32. Effect of Uncertainty in Control-Rod Channel Pitch.

\begin{tabular}{||c|c|ccc|c|ccc||}
\hline Case & Deviation & $\Delta \mathrm{k}$ & \pm & $\sigma_{\Delta \mathrm{k}}$ & $\begin{array}{c}\text { Scaling } \\
\text { Factor }\end{array}$ & $\Delta \mathrm{k}_{\text {eff }}(1 \sigma)$ & \pm & $\sigma_{\Delta \mathrm{keff}}$ \\
\hline \hline \multirow{2}{*}{1} & $-2 \mathrm{~mm}(20 \times$ limit $)$ & -0.00009 & \pm & 0.00016 & $20 \sqrt{3}$ & 0.00000 & \pm & 0.00000 \\
& $+2 \mathrm{~mm}(20 \times$ limit $)$ & -0.00004 & \pm & 0.00017 & $20 \sqrt{3}$ & 0.00000 & \pm & 0.00000 \\
\hline \multirow{2}{*}{2} & $-2 \mathrm{~mm}(20 \times$ limit $)$ & 0.00044 & \pm & 0.00017 & $20 \sqrt{3}$ & 0.00001 & \pm & 0.00000 \\
& $+2 \mathrm{~mm}(20 \times$ limit $)$ & 0.00010 & \pm & 0.00016 & $20 \sqrt{3}$ & 0.00000 & \pm & 0.00000 \\
\hline \multirow{3}{*}{3} & $-2 \mathrm{~mm}(20 \times$ limit $)$ & -0.00005 & \pm & 0.00017 & $20 \sqrt{3}$ & 0.00000 & \pm & 0.00000 \\
& $+2 \mathrm{~mm}(20 \times$ limit $)$ & -0.00023 & \pm & 0.00017 & $20 \sqrt{3}$ & -0.00001 & \pm & 0.00000 \\
4 & $-2 \mathrm{~mm}(20 \times$ limit $)$ & -0.00007 & \pm & 0.00017 & $20 \sqrt{3}$ & 0.00000 & \pm & 0.00000 \\
& $+2 \mathrm{~mm}(20 \times$ limit $)$ & -0.00031 & \pm & 0.00017 & $20 \sqrt{3}$ & -0.00001 & \pm & 0.00000 \\
\hline \multirow{3}{*}{5} & $-2 \mathrm{~mm}(20 \times$ limit $)$ & -0.00002 & \pm & 0.00016 & $20 \sqrt{3}$ & 0.00000 & \pm & 0.00000 \\
& $+2 \mathrm{~mm}(20 \times$ limit $)$ & 0.00012 & \pm & 0.00016 & $20 \sqrt{3}$ & 0.00000 & \pm & 0.00000 \\
\hline
\end{tabular}

\subsubsection{Permanent Reflectors}

Insufficient information is available to model in detail the permanent reflector of the HTTR. A bias was not assessed for any simplification of the permanent reflector. The actual reflector is in the shape of a dodecagon block with an overall diameter and length of $4250 \mathrm{~mm}$ and $5250 \mathrm{~mm}$, respectively. It is unclear as to whether the diameter is inscribed within or circumscribed around the polygon.

A radial representation of the permanent reflector had the outer diameter varied $\pm 10 \mathrm{~cm}$ to determine the effective change in $\Delta \mathrm{k}$. The difference between the reported diameter and an equivalent diameter circle representative of an inscribed or circumscribed dodecagon would by $-10 \mathrm{~cm}$ and $+5 \mathrm{~cm}$, respectively. This uncertainty is treated as a bounding uncertainty. Any uncertainty in the unreported manufacturing tolerances would be negligible. Results are shown in Table 2.33.

The permanent reflector is comprised of 12 circumferential segments in eight axial layers for a total of 96 blocks. However, the uncertainty in the diameter of the model's permanent reflector is not adjusted for random uncertainty and treated as $100 \%$ systematic because of the uncertainty in the overall detail of the permanent reflector. 
NEA/NSC/DOC(2006)1

Gas Cooled (Thermal) Reactor - GCR

HTTR-GCR-RESR-002

CRIT-REAC-RRATE

Table 2.33. Effect of Uncertainty in Permanent Reflector Diameter.

\begin{tabular}{||c|c|ccc|c|ccc||}
\hline \hline Case & Deviation & $\Delta \mathrm{k}$ & \pm & $\sigma_{\Delta \mathrm{k}}$ & $\begin{array}{c}\text { Scaling } \\
\text { Factor }\end{array}$ & $\Delta \mathrm{k}_{\text {eff }}(1 \sigma)$ & \pm & $\sigma_{\Delta \mathrm{keff}}$ \\
\hline \hline \multirow{2}{*}{1} & $-10 \mathrm{~cm}$ & -0.00202 & \pm & 0.00017 & $\sqrt{ } 3$ & -0.00117 & \pm & 0.00010 \\
& $+10 \mathrm{~cm}$ & 0.00155 & \pm & 0.00016 & $2 \sqrt{3}$ & 0.00045 & \pm & 0.00005 \\
\hline \multirow{2}{*}{2} & $-10 \mathrm{~cm}$ & -0.00144 & \pm & 0.00016 & $\sqrt{ } 3$ & -0.00083 & \pm & 0.00009 \\
& $+10 \mathrm{~cm}$ & 0.00150 & \pm & 0.00017 & $2 \sqrt{3}$ & 0.00043 & \pm & 0.00005 \\
\hline \multirow{3}{*}{3} & $-10 \mathrm{~cm}$ & -0.00145 & \pm & 0.00016 & $\sqrt{3}$ & -0.00084 & \pm & 0.00009 \\
& $+10 \mathrm{~cm}$ & 0.00119 & \pm & 0.00016 & $2 \sqrt{3}$ & 0.00034 & \pm & 0.00005 \\
4 & $-10 \mathrm{~cm}$ & -0.00093 & \pm & 0.00017 & $\sqrt{3}$ & -0.00054 & \pm & 0.00010 \\
& $+10 \mathrm{~cm}$ & 0.00050 & \pm & 0.00017 & $2 \sqrt{3}$ & 0.00014 & \pm & 0.00005 \\
\hline \multirow{2}{*}{5} & $-10 \mathrm{~cm}$ & -0.00144 & \pm & 0.00016 & $\sqrt{3}$ & -0.00083 & \pm & 0.00009 \\
& $+10 \mathrm{~cm}$ & 0.00083 & \pm & 0.00016 & $2 \sqrt{3}$ & 0.00024 & \pm & 0.00005 \\
\hline
\end{tabular}

\subsubsection{Dummy Blocks}

The uncertainty in the outer dimensions of the dummy blocks was included with the graphite block analysis in Section 2.1.2.7. There are two types of dummy blocks, one with a hole pattern similar to that of a control block, and the other with three holes but of smaller diameter (Ref. 2, p. 14). Because the true dimensions of the smaller hole design is unknown, the dummy blocks will all be modeled with the holes of the control blocks but an additional uncertainty will be added to account for this discrepancy. This uncertainty will be assessed by completely filling the holes of the dummy blocks with IG-11 graphite material (Section 2.1.3.9).

\subsubsection{Compositional Variations}

\subsubsection{Coated Fuel Particles}

\section{Uranium Enrichment}

The concentration of ${ }^{234} U$ expected in the TRISO fuel had to be determined, as it was not provided. First the weight fractions of isotopes in natural uranium dioxide were determined. Then the enriched weight percent of ${ }^{235} \mathrm{U}$ was multiplied by the natural weight percent of ${ }^{234} \mathrm{U}(0.0055$ at.\%) and divided by the natural weight percent of ${ }^{235} \mathrm{U}\left(0.72\right.$ at.\%). Thus an approximate concentration of "enriched" ${ }^{234} \mathrm{U}$ content could be determined for this evaluation, which may slightly underestimate the actual ${ }^{234} \mathrm{U}$ content.

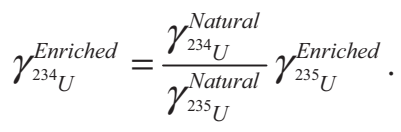

Information was not provided regarding the uncertainty in the uranium enrichment of the TRISO kernels. It is reported elsewhere that the manufacturing tolerance limit for the enrichment is $4.5 \%$ of the reported weight percent. ${ }^{a}$ For example, the enrichment of $3.4 \mathrm{wt} . \%$ is bound within a tolerance of $\pm 0.153 \mathrm{wt} . \%$.

\footnotetext{
${ }^{a}$ S. Maruyama, K. Yamashita, N. Fujimoto, I. Murata, R. Shindo, and Y. Sudo, "Determination of Hot Spot Factors for Calculation of the Maximum Fuel Temperatures in the Core Thermal and Hydraulic Design of HTTR," JAERIM 88-250, JAEA (November 18, 1988). [in Japanese].
} 
NEA/NSC/DOC(2006)1

\section{Gas Cooled (Thermal) Reactor - GCR \\ HTTR-GCR-RESR-002 \\ CRIT-REAC-RRATE}

The ${ }^{234} \mathrm{U}$ content adjusted to match the effective increase or decrease in enrichment of ${ }^{235} \mathrm{U}$, to determine the effective uncertainty in $\mathrm{k}_{\text {eff. }}$ The nominal enrichment values are shown in Figure 1.46 and Table 1.11 of HTTR-GCR-RESR-001. Results are shown in Tables 2.34 through 2.38. The actual uncertainty in the uranium enrichment is much smaller than the manufacturing limits; however this information is not publicly available. Therefore, the bounding limits are treated with a normal distribution instead of one with uniform probability.

Configurations 1 through 4 do not contain uranium fuel with the enrichments of 3.40 and $6.70 \mathrm{wt} . \%$.

The uncertainty in the uranium enrichment is considered all systematic with no random component.

Table 2.34. Effect of Uncertainty in Uranium Enrichment (Case 1).

\begin{tabular}{|c|c|c|c|c|c|c|c|}
\hline Deviation & $\Delta \mathrm{k}$ & \pm & $\sigma_{\Delta \mathrm{k}}$ & $\begin{array}{l}\text { Scaling } \\
\text { Factor }\end{array}$ & $\Delta \mathrm{k}_{\mathrm{eff}}(1 \sigma)$ & \pm & $\sigma_{\Delta \mathrm{keff}}$ \\
\hline-0.1755 wt. $\%$ of 3.9 wt. $\%$ & -0.00012 & \pm & 0.00016 & 3 & -0.00004 & \pm & 0.00005 \\
\hline+0.1755 wt. $\%$ of 3.9 wt. $\%$ & 0.00035 & \pm & 0.00016 & 3 & 0.00012 & \pm & 0.00005 \\
\hline-0.1935 wt. $\%$ of 4.3 wt. $\%$ & -0.00163 & \pm & 0.00016 & 3 & -0.00054 & \pm & 0.00005 \\
\hline+0.1935 wt. $\%$ of 4.3 wt. $\%$ & 0.00162 & \pm & 0.00016 & 3 & 0.00054 & \pm & 0.00005 \\
\hline-0.216 wt. $\%$ of 4.8 wt. $\%$ & -0.00069 & \pm & 0.00016 & 3 & -0.00023 & \pm & 0.00005 \\
\hline+0.216 wt. $\%$ of 4.8 wt. $\%$ & 0.00066 & \pm & 0.00016 & 3 & 0.00022 & \pm & 0.00005 \\
\hline-0.234 wt. $\%$ of 5.2 wt. $\%$ & -0.00018 & \pm & 0.00016 & 3 & -0.00006 & \pm & 0.00005 \\
\hline+0.234 wt. $\%$ of 5.2 wt. $\%$ & 0.00023 & \pm & 0.00016 & 3 & 0.00008 & \pm & 0.00005 \\
\hline-0.2655 wt. $\%$ of 5.9 wt. $\%$ & -0.00189 & \pm & 0.00016 & 3 & -0.00063 & \pm & 0.00005 \\
\hline+0.2655 wt. $\%$ of 5.9 wt. $\%$ & 0.00180 & \pm & 0.00016 & 3 & 0.00060 & \pm & 0.00005 \\
\hline-0.2835 wt. $\%$ of 6.3 wt. $\%$ & -0.00127 & \pm & 0.00016 & 3 & -0.00042 & \pm & 0.00005 \\
\hline+0.2835 wt. $\%$ of 6.3 wt. $\%$ & 0.00101 & \pm & 0.00016 & 3 & 0.00034 & \pm & 0.00005 \\
\hline-0.324 wt. $\%$ of 7.2 wt. $\%$ & -0.00221 & \pm & 0.00016 & 3 & -0.00074 & \pm & 0.00005 \\
\hline+0.324 wt. $\%$ of 7.2 wt. $\%$ & 0.00238 & \pm & 0.00016 & 3 & 0.00079 & \pm & 0.00005 \\
\hline-0.3555 wt. $\%$ of 7.9 wt. $\%$ & -0.00114 & \pm & 0.00016 & 3 & -0.00038 & \pm & 0.00005 \\
\hline+0.3555 wt. $\%$ of 7.9 wt. $\%$ & 0.00143 & \pm & 0.00016 & 3 & 0.00048 & \pm & 0.00005 \\
\hline-0.423 wt. $\%$ of 9.4 wt. $\%$ & -0.00166 & \pm & 0.00016 & 3 & -0.00055 & \pm & 0.00005 \\
\hline+0.423 wt. $\%$ of 9.4 wt. $\%$ & 0.00186 & \pm & 0.00016 & 3 & 0.00062 & \pm & 0.00005 \\
\hline-0.4455 wt. $\%$ of 9.9 wt. $\%$ & -0.00093 & \pm & 0.00016 & 3 & -0.00031 & \pm & 0.00005 \\
\hline+0.4455 wt. $\%$ of 9.9 wt. $\%$ & 0.00081 & \pm & 0.00016 & 3 & 0.00027 & \pm & 0.00005 \\
\hline
\end{tabular}


NEA/NSC/DOC(2006)1

Gas Cooled (Thermal) Reactor - GCR

HTTR-GCR-RESR-002

CRIT-REAC-RRATE

Table 2.35. Effect of Uncertainty in Uranium Enrichment (Case 2).

\begin{tabular}{|c|c|c|c|c|c|c|c|}
\hline Deviation & $\Delta \mathrm{k}$ & \pm & $\sigma_{\Delta \mathrm{k}}$ & $\begin{array}{l}\text { Scaling } \\
\text { Factor }\end{array}$ & $\Delta \mathrm{k}_{\mathrm{eff}}(1 \sigma)$ & \pm & $\sigma_{\Delta \mathrm{keff}}$ \\
\hline-0.1755 wt. $\%$ of 3.9 wt. $\%$ & -0.00091 & \pm & 0.00016 & 3 & -0.00030 & \pm & 0.00005 \\
\hline+0.1755 wt. $\%$ of 3.9 wt. $\%$ & 0.00068 & \pm & 0.00016 & 3 & 0.00023 & \pm & 0.00005 \\
\hline-0.1935 wt. $\%$ of 4.3 wt. $\%$ & -0.00244 & \pm & 0.00016 & 3 & -0.00081 & \pm & 0.00005 \\
\hline+0.1935 wt. $\%$ of 4.3 wt. $\%$ & 0.00220 & \pm & 0.00016 & 3 & 0.00073 & \pm & 0.00005 \\
\hline-0.216 wt. $\%$ of 4.8 wt. $\%$ & -0.00124 & \pm & 0.00016 & 3 & -0.00041 & \pm & 0.00005 \\
\hline+0.216 wt. $\%$ of 4.8 wt. $\%$ & 0.00110 & \pm & 0.00016 & 3 & 0.00037 & \pm & 0.00005 \\
\hline-0.234 wt. $\%$ of 5.2 wt. $\%$ & -0.00099 & \pm & 0.00016 & 3 & -0.00033 & \pm & 0.00005 \\
\hline+0.234 wt. $\%$ of 5.2 wt. $\%$ & 0.00051 & \pm & 0.00016 & 3 & 0.00017 & \pm & 0.00005 \\
\hline-0.2655 wt. $\%$ of 5.9 wt. $\%$ & -0.00229 & \pm & 0.00016 & 3 & -0.00076 & \pm & 0.00005 \\
\hline+0.2655 wt. $\%$ of 5.9 wt. $\%$ & 0.00227 & \pm & 0.00016 & 3 & 0.00076 & \pm & 0.00005 \\
\hline-0.2835 wt. $\%$ of 6.3 wt. $\%$ & -0.00202 & \pm & 0.00016 & 3 & -0.00067 & \pm & 0.00005 \\
\hline+0.2835 wt. $\%$ of 6.3 wt. $\%$ & 0.00140 & \pm & 0.00016 & 3 & 0.00047 & \pm & 0.00005 \\
\hline-0.324 wt. $\%$ of 7.2 wt. $\%$ & -0.00213 & \pm & 0.00016 & 3 & -0.00071 & \pm & 0.00005 \\
\hline+0.324 wt. $\%$ of 7.2 wt. $\%$ & 0.00180 & \pm & 0.00016 & 3 & 0.00060 & \pm & 0.00005 \\
\hline-0.3555 wt. $\%$ of 7.9 wt. $\%$ & -0.00114 & \pm & 0.00016 & 3 & -0.00038 & \pm & 0.00005 \\
\hline+0.3555 wt. $\%$ of 7.9 wt. $\%$ & 0.00093 & \pm & 0.00016 & 3 & 0.00031 & \pm & 0.00005 \\
\hline-0.423 wt. $\%$ of 9.4 wt. $\%$ & -0.00078 & \pm & 0.00016 & 3 & -0.00026 & \pm & 0.00005 \\
\hline+0.423 wt. $\%$ of 9.4 wt. $\%$ & 0.00048 & \pm & 0.00016 & 3 & 0.00016 & \pm & 0.00005 \\
\hline-0.4455 wt. $\%$ of 9.9 wt. $\%$ & -0.00044 & \pm & 0.00016 & 3 & -0.00015 & \pm & 0.00005 \\
\hline+0.4455 wt. $\%$ of 9.9 wt. $\%$ & 0.00013 & \pm & 0.00016 & 3 & 0.00004 & \pm & 0.00005 \\
\hline
\end{tabular}


NEA/NSC/DOC(2006)1

Gas Cooled (Thermal) Reactor - GCR

HTTR-GCR-RESR-002

CRIT-REAC-RRATE

Table 2.36. Effect of Uncertainty in Uranium Enrichment (Case 3).

\begin{tabular}{|c|c|c|c|c|c|c|c|}
\hline Deviation & $\Delta \mathrm{k}$ & \pm & $\sigma_{\Delta \mathrm{k}}$ & $\begin{array}{l}\text { Scaling } \\
\text { Factor }\end{array}$ & $\Delta \mathrm{k}_{\mathrm{eff}}(1 \sigma)$ & \pm & $\sigma_{\Delta \mathrm{keff}}$ \\
\hline-0.1755 wt. $\%$ of 3.9 wt. $\%$ & -0.00193 & \pm & 0.00016 & 3 & -0.00064 & \pm & 0.00005 \\
\hline+0.1755 wt. $\%$ of 3.9 wt. $\%$ & 0.00231 & \pm & 0.00016 & 3 & 0.00077 & \pm & 0.00005 \\
\hline-0.1935 wt. $\%$ of 4.3 wt. $\%$ & -0.00277 & \pm & 0.00016 & 3 & -0.00092 & \pm & 0.00005 \\
\hline+0.1935 wt. $\%$ of 4.3 wt. $\%$ & 0.00293 & \pm & 0.00016 & 3 & 0.00098 & \pm & 0.00005 \\
\hline-0.216 wt. $\%$ of 4.8 wt. $\%$ & -0.00130 & \pm & 0.00016 & 3 & -0.00043 & \pm & 0.00005 \\
\hline+0.216 wt. $\%$ of 4.8 wt. $\%$ & 0.00157 & \pm & 0.00016 & 3 & 0.00052 & \pm & 0.00005 \\
\hline-0.234 wt. $\%$ of 5.2 wt. $\%$ & -0.00137 & \pm & 0.00016 & 3 & -0.00046 & \pm & 0.00005 \\
\hline+0.234 wt. $\%$ of 5.2 wt. $\%$ & 0.00148 & \pm & 0.00016 & 3 & 0.00049 & \pm & 0.00005 \\
\hline-0.2655 wt. $\%$ of 5.9 wt. $\%$ & -0.00175 & \pm & 0.00016 & 3 & -0.00058 & \pm & 0.00005 \\
\hline+0.2655 wt. $\%$ of 5.9 wt. $\%$ & 0.00212 & \pm & 0.00016 & 3 & 0.00071 & \pm & 0.00005 \\
\hline-0.2835 wt. $\%$ of 6.3 wt. $\%$ & -0.00135 & \pm & 0.00016 & 3 & -0.00045 & \pm & 0.00005 \\
\hline+0.2835 wt. $\%$ of 6.3 wt. $\%$ & 0.00155 & \pm & 0.00016 & 3 & 0.00052 & \pm & 0.00005 \\
\hline-0.324 wt. $\%$ of 7.2 wt. $\%$ & -0.00094 & \pm & 0.00016 & 3 & -0.00031 & \pm & 0.00005 \\
\hline+0.324 wt. $\%$ of 7.2 wt. $\%$ & 0.00108 & \pm & 0.00016 & 3 & 0.00036 & \pm & 0.00005 \\
\hline-0.3555 wt. $\%$ of 7.9 wt. $\%$ & -0.00034 & \pm & 0.00016 & 3 & -0.00011 & \pm & 0.00005 \\
\hline+0.3555 wt. $\%$ of 7.9 wt. $\%$ & 0.00051 & \pm & 0.00016 & 3 & 0.00017 & \pm & 0.00005 \\
\hline-0.423 wt. $\%$ of 9.4 wt. $\%$ & -0.00008 & \pm & 0.00016 & 3 & -0.00003 & \pm & 0.00005 \\
\hline+0.423 wt. $\%$ of 9.4 wt. $\%$ & 0.00039 & \pm & 0.00016 & 3 & 0.00013 & \pm & 0.00005 \\
\hline-0.4455 wt. $\%$ of 9.9 wt. $\%$ & 0.00003 & \pm & 0.00016 & 3 & 0.00001 & \pm & 0.00005 \\
\hline+0.4455 wt. $\%$ of 9.9 wt. $\%$ & 0.00003 & \pm & 0.00016 & 3 & 0.00001 & \pm & 0.00005 \\
\hline
\end{tabular}


NEA/NSC/DOC(2006)1

Gas Cooled (Thermal) Reactor - GCR

HTTR-GCR-RESR-002

CRIT-REAC-RRATE

Table 2.37. Effect of Uncertainty in Uranium Enrichment (Case 4).

\begin{tabular}{|c|c|c|c|c|c|c|c|}
\hline Deviation & $\Delta \mathrm{k}$ & \pm & $\sigma_{\Delta \mathrm{k}}$ & $\begin{array}{l}\text { Scaling } \\
\text { Factor }\end{array}$ & $\Delta \mathrm{k}_{\mathrm{eff}}(1 \sigma)$ & \pm & $\sigma_{\Delta \mathrm{keff}}$ \\
\hline-0.1755 wt. $\%$ of 3.9 wt. $\%$ & -0.00159 & \pm & 0.00017 & 3 & -0.00053 & \pm & 0.00006 \\
\hline+0.1755 wt. $\%$ of 3.9 wt. $\%$ & 0.00176 & \pm & 0.00017 & 3 & 0.00059 & \pm & 0.00006 \\
\hline-0.1935 wt. $\%$ of 4.3 wt. $\%$ & -0.00193 & \pm & 0.00017 & 3 & -0.00064 & \pm & 0.00006 \\
\hline+0.1935 wt. $\%$ of 4.3 wt. $\%$ & 0.00238 & \pm & 0.00016 & 3 & 0.00079 & \pm & 0.00005 \\
\hline-0.216 wt. $\%$ of 4.8 wt. $\%$ & -0.00091 & \pm & 0.00017 & 3 & -0.00030 & \pm & 0.00006 \\
\hline+0.216 wt. $\%$ of 4.8 wt. $\%$ & 0.00108 & \pm & 0.00017 & 3 & 0.00036 & \pm & 0.00006 \\
\hline-0.234 wt. $\%$ of 5.2 wt. $\%$ & -0.00135 & \pm & 0.00017 & 3 & -0.00045 & \pm & 0.00006 \\
\hline+0.234 wt. $\%$ of 5.2 wt. $\%$ & 0.00154 & \pm & 0.00017 & 3 & 0.00051 & \pm & 0.00006 \\
\hline-0.2655 wt. $\%$ of 5.9 wt. $\%$ & -0.00145 & \pm & 0.00017 & 3 & -0.00048 & \pm & 0.00006 \\
\hline+0.2655 wt. $\%$ of 5.9 wt. $\%$ & 0.00124 & \pm & 0.00017 & 3 & 0.00041 & \pm & 0.00006 \\
\hline-0.2835 wt. $\%$ of 6.3 wt. $\%$ & -0.00190 & \pm & 0.00017 & 3 & -0.00063 & \pm & 0.00006 \\
\hline+0.2835 wt. $\%$ of 6.3 wt. $\%$ & 0.00197 & \pm & 0.00017 & 3 & 0.00066 & \pm & 0.00006 \\
\hline-0.324 wt. $\%$ of 7.2 wt. $\%$ & -0.00103 & \pm & 0.00016 & 3 & -0.00034 & \pm & 0.00005 \\
\hline+0.324 wt. $\%$ of 7.2 wt. $\%$ & 0.00099 & \pm & 0.00017 & 3 & 0.00033 & \pm & 0.00006 \\
\hline-0.3555 wt. $\%$ of 7.9 wt. $\%$ & -0.00112 & \pm & 0.00017 & 3 & -0.00037 & \pm & 0.00006 \\
\hline+0.3555 wt. $\%$ of 7.9 wt. $\%$ & 0.00133 & \pm & 0.00016 & 3 & 0.00044 & \pm & 0.00005 \\
\hline-0.423 wt. $\%$ of 9.4 wt. $\%$ & -0.00042 & \pm & 0.00016 & 3 & -0.00014 & \pm & 0.00005 \\
\hline+0.423 wt. $\%$ of 9.4 wt. $\%$ & 0.00055 & \pm & 0.00017 & 3 & 0.00018 & \pm & 0.00006 \\
\hline-0.4455 wt. $\%$ of 9.9 wt. $\%$ & -0.00013 & \pm & 0.00017 & 3 & -0.00004 & \pm & 0.00006 \\
\hline+0.4455 wt. $\%$ of 9.9 wt. $\%$ & 0.00032 & \pm & 0.00017 & 3 & 0.00011 & \pm & 0.00006 \\
\hline
\end{tabular}


NEA/NSC/DOC(2006)1

Gas Cooled (Thermal) Reactor - GCR

HTTR-GCR-RESR-002

CRIT-REAC-RRATE

Table 2.38. Effect of Uncertainty in Uranium Enrichment (Case 5).

\begin{tabular}{|c|c|c|c|c|c|c|c|}
\hline Deviation & $\Delta \mathrm{k}$ & \pm & $\sigma_{\Delta \mathrm{k}}$ & $\begin{array}{l}\text { Scaling } \\
\text { Factor }\end{array}$ & $\Delta \mathrm{k}_{\mathrm{eff}}(1 \sigma)$ & \pm & $\sigma_{\Delta \mathrm{keff}}$ \\
\hline-0.153 wt. $\%$ of 3.4 wt. $\%$ & -0.00162 & \pm & 0.00017 & 3 & -0.00054 & \pm & 0.00006 \\
\hline+0.153 wt. $\%$ of 3.4 wt. $\%$ & 0.00125 & \pm & 0.00016 & 3 & 0.00042 & \pm & 0.00005 \\
\hline-0.1755 wt. $\%$ of 3.9 wt. $\%$ & -0.00238 & \pm & 0.00016 & 3 & -0.00079 & \pm & 0.00005 \\
\hline+0.1755 wt. $\%$ of 3.9 wt. $\%$ & 0.00238 & \pm & 0.00017 & 3 & 0.00079 & \pm & 0.00006 \\
\hline-0.1935 wt. $\%$ of 4.3 wt. $\%$ & -0.00399 & \pm & 0.00016 & 3 & -0.00133 & \pm & 0.00005 \\
\hline+0.1935 wt. $\%$ of 4.3 wt. $\%$ & 0.00398 & \pm & 0.00017 & 3 & 0.00133 & \pm & 0.00006 \\
\hline-0.216 wt. $\%$ of 4.8 wt. $\%$ & -0.00155 & \pm & 0.00017 & 3 & -0.00052 & \pm & 0.00006 \\
\hline+0.216 wt. $\%$ of 4.8 wt. $\%$ & 0.00152 & \pm & 0.00016 & 3 & 0.00051 & \pm & 0.00005 \\
\hline-0.234 wt. $\%$ of 5.2 wt. $\%$ & -0.00118 & \pm & 0.00017 & 3 & -0.00039 & \pm & 0.00006 \\
\hline+0.234 wt. $\%$ of 5.2 wt. $\%$ & 0.00111 & \pm & 0.00016 & 3 & 0.00037 & \pm & 0.00005 \\
\hline-0.2655 wt. $\%$ of 5.9 wt. $\%$ & -0.00157 & \pm & 0.00017 & 3 & -0.00052 & \pm & 0.00006 \\
\hline+0.2655 wt. $\%$ of 5.9 wt. $\%$ & 0.00142 & \pm & 0.00016 & 3 & 0.00047 & \pm & 0.00005 \\
\hline-0.2835 wt. $\%$ of 6.3 wt. $\%$ & -0.00091 & \pm & 0.00016 & 3 & -0.00030 & \pm & 0.00005 \\
\hline+0.2835 wt. $\%$ of 6.3 wt. $\%$ & 0.00078 & \pm & 0.00016 & 3 & 0.00026 & \pm & 0.00005 \\
\hline-0.3015 wt. $\%$ of 6.7 wt. $\%$ & 0.00009 & \pm & 0.00017 & 3 & 0.00003 & \pm & 0.00006 \\
\hline+0.3015 wt. $\%$ of 6.7 wt. $\%$ & 0.00007 & \pm & 0.00016 & 3 & 0.00002 & \pm & 0.00005 \\
\hline-0.324 wt. $\%$ of 7.2 wt. $\%$ & -0.00037 & \pm & 0.00017 & 3 & -0.00012 & \pm & 0.00006 \\
\hline+0.324 wt. $\%$ of 7.2 wt. $\%$ & 0.00033 & \pm & 0.00017 & 3 & 0.00011 & \pm & 0.00006 \\
\hline-0.3555 wt. $\%$ of 7.9 wt. $\%$ & -0.00025 & \pm & 0.00017 & 3 & -0.00008 & \pm & 0.00006 \\
\hline+0.3555 wt. $\%$ of 7.9 wt. $\%$ & 0.00019 & \pm & 0.00016 & 3 & 0.00006 & \pm & 0.00005 \\
\hline-0.423 wt. $\%$ of 9.4 wt. $\%$ & 0.00003 & \pm & 0.00017 & 3 & 0.00001 & \pm & 0.00006 \\
\hline+0.423 wt. $\%$ of 9.4 wt. $\%$ & -0.00005 & \pm & 0.00016 & 3 & -0.00002 & \pm & 0.00005 \\
\hline-0.4455 wt. $\%$ of 9.9 wt. $\%$ & -0.00002 & \pm & 0.00017 & 3 & -0.00001 & \pm & 0.00006 \\
\hline+0.4455 wt. $\%$ of 9.9 wt. $\%$ & 0.00022 & \pm & 0.00016 & 3 & 0.00007 & \pm & 0.00005 \\
\hline
\end{tabular}

\section{Oxygen to Uranium Ratio}

The oxygen to uranium ratio was varied by a best judgment value of $\pm 0.06(3 \times$ bounding limit $)$ from the nominal value of 2.00 to determine the effective uncertainty in $\mathrm{k}_{\mathrm{eff}}$. Results are shown in Table 2.39.

The uncertainty in the oxygen to uranium ratio is considered all systematic with no random component. 
NEA/NSC/DOC(2006)1

Gas Cooled (Thermal) Reactor - GCR

HTTR-GCR-RESR-002

CRIT-REAC-RRATE

Table 2.39. Effect of Uncertainty in Oxygen to Uranium Ratio.

\begin{tabular}{|c|c|c|c|c|c|c|c|c|c|}
\hline Case & Deviation & $\Delta \mathrm{k}$ & \pm & $\sigma_{\Delta \mathrm{k}}$ & $\begin{array}{l}\text { Scaling } \\
\text { Factor }\end{array}$ & $\Delta \mathrm{k}_{\mathrm{eff}}(1 \sigma)$ & \pm & $\sigma_{\Delta \mathrm{keff}}$ & $\mathrm{N}$ \\
\hline \multirow{2}{*}{1} & -0.06 & 0.00044 & \pm & 0.00017 & $3 \sqrt{3}$ & 0.00008 & \pm & 0.00003 & \multirow{2}{*}{$5.38 \times 10^{8}$} \\
\hline & +0.06 & -0.00047 & \pm & 0.00016 & $3 \sqrt{3}$ & -0.00009 & \pm & 0.0003 & \\
\hline \multirow{2}{*}{2} & -0.06 & 0.00074 & \pm & 0.00016 & $3 \sqrt{3}$ & 0.00014 & \pm & 0.00003 & \multirow{2}{*}{$5.98 \times 10^{8}$} \\
\hline & +0.06 & -0.00036 & \pm & 0.00016 & $3 \sqrt{3}$ & -0.00007 & \pm & 0.00003 & \\
\hline \multirow{2}{*}{3} & -0.06 & 0.00035 & \pm & 0.00016 & $3 \sqrt{3}$ & 0.00007 & \pm & 0.00003 & \multirow{2}{*}{$6.88 \times 10^{8}$} \\
\hline & +0.06 & -0.00086 & \pm & 0.00017 & $3 \sqrt{3}$ & -0.00017 & \pm & 0.00003 & \\
\hline \multirow{2}{*}{4} & -0.06 & 0.00055 & \pm & 0.00016 & $3 \sqrt{3}$ & 0.00011 & \pm & 0.00003 & \multirow{2}{*}{$6.88 \times 10^{8}$} \\
\hline & +0.06 & -0.00060 & \pm & 0.00017 & $3 \sqrt{3}$ & -0.00012 & \pm & 0.00003 & \\
\hline \multirow{2}{*}{5} & -0.06 & 0.00048 & \pm & 0.00016 & $3 \sqrt{3}$ & 0.00009 & \pm & 0.00003 & \multirow{2}{*}{$7.78 \times 10^{8}$} \\
\hline & +0.06 & -0.00063 & \pm & 0.00016 & $3 \sqrt{3}$ & -0.00012 & \pm & 0.00003 & \\
\hline
\end{tabular}

\section{$\underline{\mathrm{UO}}_{2} \underline{\text { Density }}$}

Because of the overspecification of the TRISO particles in Table 1.14 (HTTR-GCR-RESR-001) and the correlation of uranium kernel diameter, density, TRISO packing fraction, and mass, the effect of the uncertainty in the fuel density is not included in the total uncertainty. However, an analysis of the uncertainty based upon the fuel mass is performed in Section 2.1.6.

\section{$\underline{\mathrm{UO}}_{2} \underline{\text { Impurity }}$}

The kernel impurity was varied from $0-3$ ppm by weight of equivalent natural-boron content to determine the bounding uncertainty in $\mathrm{k}_{\mathrm{eff}}$. The maximum limit was multiplied 10 -fold so as to quantify the effective upper uncertainty in the $\mathrm{UO}_{2}$ impurity. The average value is $1.5 \mathrm{ppm}$ by weight (Tables 1.13 and 1.14 of HTTR-GCR-RESR-001). Results are shown in Table 2.40.

The uncertainty in the $\mathrm{UO}_{2}$ impurity is considered all systematic with no random component. 
NEA/NSC/DOC(2006)1

Gas Cooled (Thermal) Reactor - GCR

HTTR-GCR-RESR-002

CRIT-REAC-RRATE

Table 2.40. Effect of Uncertainty in $\mathrm{UO}_{2}$ Impurity.

\begin{tabular}{|c|c|ccc|c|ccc||}
\hline Case & Deviation & $\Delta \mathrm{k}$ & \pm & $\sigma_{\Delta \mathrm{k}}$ & $\begin{array}{c}\text { Scaling } \\
\text { Factor }\end{array}$ & $\Delta \mathrm{k}_{\text {eff }}(1 \sigma)$ & \pm & $\sigma_{\Delta \mathrm{keff}}$ \\
\hline \hline \multirow{2}{*}{1} & $0 \mathrm{ppm}$ & 0.00055 & \pm & 0.00016 & $\sqrt{ } 3$ & 0.00032 & \pm & 0.00009 \\
& $30 \mathrm{ppm}$ & -0.00963 & \pm & 0.00017 & $10 \sqrt{3}$ & -0.00056 & \pm & 0.00001 \\
\hline \multirow{2}{*}{2} & $0 \mathrm{ppm}$ & 0.00081 & \pm & 0.00016 & $\sqrt{ } 3$ & 0.00047 & \pm & 0.00009 \\
& $30 \mathrm{ppm}$ & -0.01034 & \pm & 0.00017 & $10 \sqrt{3}$ & -0.00060 & \pm & 0.00001 \\
\hline \multirow{2}{*}{3} & $0 \mathrm{ppm}$ & 0.00042 & \pm & 0.00016 & $\sqrt{ } 3$ & 0.00024 & \pm & 0.00009 \\
& $30 \mathrm{ppm}$ & -0.01176 & \pm & 0.00016 & $10 \sqrt{3}$ & -0.00068 & \pm & 0.00001 \\
\hline \multirow{3}{*}{4} & $0 \mathrm{ppm}$ & 0.00045 & \pm & 0.00016 & $\sqrt{ } 3$ & 0.00026 & \pm & 0.00009 \\
& $30 \mathrm{ppm}$ & -0.01090 & \pm & 0.00016 & $10 \sqrt{3}$ & -0.00063 & \pm & 0.00001 \\
\hline \multirow{2}{*}{5} & $0 \mathrm{ppm}$ & 0.00082 & \pm & 0.00016 & $\sqrt{ } 3$ & 0.00047 & \pm & 0.00009 \\
& $30 \mathrm{ppm}$ & -0.01260 & \pm & 0.00016 & $10 \sqrt{3}$ & -0.00073 & \pm & 0.00001 \\
\hline
\end{tabular}

\section{Buffer Density}

The buffer density was varied $\pm 0.30 \mathrm{~g} / \mathrm{cm}^{3}$ from the nominal value of $1.10 \mathrm{~g} / \mathrm{cm}^{3}$ (Table 1.14 of HTTR-GCR-RESR-001) to determine the effective uncertainty in $\mathrm{k}_{\text {eff }}$. This value is three times the bounding limit. Results are shown in Table 2.41.

The total number of TRISO particles used in the fully-loaded core is approximately $868,140,000$. For determining the random component of the uncertainty, the results in Table 2.41 would be divided by $\sqrt{ } \mathrm{N}$, where $\mathrm{N}$ for each case is shown in Table 2.41.

Table 2.41. Effect of Uncertainty in Buffer Density.

\begin{tabular}{|c|c|c|c|c|c|c|c|c|c|}
\hline Case & Deviation & $\Delta \mathrm{k}$ & \pm & $\sigma_{\Delta \mathrm{k}}$ & $\begin{array}{l}\text { Scaling } \\
\text { Factor }\end{array}$ & $\Delta \mathrm{k}_{\mathrm{eff}}(1 \sigma)$ & \pm & $\sigma_{\Delta \mathrm{keff}}$ & $\mathrm{N}$ \\
\hline & -0.30 & -0.00005 & & 0.00017 & $3 \sqrt{3}$ & -0.00001 & \pm & 0.00003 & \multirow{2}{*}{$5.38 \times 10^{8}$} \\
\hline & $+0.30 \mathrm{~g} / \mathrm{cm}^{3}$ & 0.00014 & & 0.00017 & $3 \sqrt{3}$ & 0.00003 & \pm & 0.00003 & \\
\hline \multirow{2}{*}{2} & $-0.30 \mathrm{~g} / \mathrm{cm}^{3}$ & -0.00009 & & 0.00017 & $3 \sqrt{3}$ & -0.00002 & \pm & 0.00003 & \multirow{2}{*}{$5.98 \times 10^{8}$} \\
\hline & $+0.30 \mathrm{~g} / \mathrm{cm}^{3}$ & 0 & & 6 & $\sqrt{13}$ & & \pm & 33 & \\
\hline & $-0.30 \mathrm{~g} / \mathrm{cm}^{3}$ & -0.00021 & & 0.00017 & $3 \sqrt{3}$ & -0.00004 & \pm & 0.00003 & \multirow{2}{*}{$6.88 \times 10^{8}$} \\
\hline & $+0.30 \mathrm{~g} / \mathrm{cm}^{3}$ & 0.0 & & 7 & $3 \sqrt{3}$ & 0.0 & \pm & 03 & \\
\hline & $-0.30 \mathrm{~g} / \mathrm{cm}^{3}$ & -0.00001 & & 0.00017 & $3 \sqrt{3}$ & 0.00000 & \pm & 0.00003 & \multirow{2}{*}{$6.88 \times 10^{8}$} \\
\hline & $+0.30 \mathrm{~g} / \mathrm{cm}^{3}$ & -0.00019 & & 0.00016 & $3 \sqrt{3}$ & -0.00004 & \pm & 00003 & \\
\hline & $-0.30 \mathrm{~g} / \mathrm{cm}^{3}$ & -0.00002 & & 0.00016 & $3 \sqrt{3}$ & 0.00000 & \pm & 0.00003 & \multirow{2}{*}{$7.78 \times 10^{8}$} \\
\hline & $+0.30 \mathrm{~g} / \mathrm{cm}^{3}$ & 0.00007 & & 0.00016 & $3 \sqrt{3}$ & 0.00001 & \pm & 0.00003 & \\
\hline
\end{tabular}


NEA/NSC/DOC(2006)1

Gas Cooled (Thermal) Reactor - GCR

HTTR-GCR-RESR-002

CRIT-REAC-RRATE

\section{Buffer Impurity}

The buffer impurity was varied from 0-3 ppm by weight of equivalent natural-boron content to determine the bounding uncertainty in $\mathrm{k}_{\text {eff. }}$ The average value is $1.5 \mathrm{ppm}$ by weight (Table 1.13 of HTTR-GCR-RESR-001). Results are shown in Table 2.42.

The uncertainty in the buffer impurity is considered all systematic with no random component.

Table 2.42. Effect of Uncertainty in Buffer Impurity.

\begin{tabular}{||c|c|ccc|c|ccc||}
\hline \hline Case & Deviation & $\Delta \mathrm{k}$ & \pm & $\sigma_{\Delta \mathrm{k}}$ & $\begin{array}{c}\text { Scaling } \\
\text { Factor }\end{array}$ & $\Delta \mathrm{k}_{\text {eff }}(1 \sigma)$ & \pm & $\sigma_{\Delta \mathrm{keff}}$ \\
\hline \hline \multirow{2}{*}{1} & $0 \mathrm{ppm}$ & 0.00004 & \pm & 0.00017 & $\sqrt{ } 3$ & 0.00002 & \pm & 0.00010 \\
& $3 \mathrm{ppm}$ & 0.00001 & \pm & 0.00017 & $\sqrt{ } 3$ & 0.00001 & \pm & 0.00010 \\
\hline \multirow{2}{*}{2} & $0 \mathrm{ppm}$ & 0.00014 & \pm & 0.00017 & $\sqrt{ } 3$ & 0.00008 & \pm & 0.00010 \\
& $3 \mathrm{ppm}$ & 0.00000 & \pm & 0.00017 & $\sqrt{ } 3$ & 0.00000 & \pm & 0.00010 \\
\hline \multirow{2}{*}{3} & $0 \mathrm{ppm}$ & 0.00014 & \pm & 0.00016 & $\sqrt{3}$ & 0.00008 & \pm & 0.00009 \\
& $3 \mathrm{ppm}$ & -0.00021 & \pm & 0.00017 & $\sqrt{3}$ & -0.00012 & \pm & 0.00010 \\
\hline \multirow{3}{*}{4} & $0 \mathrm{ppm}$ & -0.00005 & \pm & 0.00017 & $\sqrt{3}$ & -0.00003 & \pm & 0.00010 \\
& $3 \mathrm{ppm}$ & -0.00017 & \pm & 0.00017 & $\sqrt{3}$ & -0.00010 & \pm & 0.00010 \\
\hline \multirow{2}{*}{5} & $0 \mathrm{ppm}$ & 0.00014 & \pm & 0.00016 & $\sqrt{3}$ & 0.00008 & \pm & 0.00009 \\
& $3 \mathrm{ppm}$ & -0.00020 & \pm & 0.00016 & $\sqrt{3}$ & -0.00012 & \pm & 0.00009 \\
\hline
\end{tabular}

\section{IPyC Density}

The IPyC density was varied +0.30 and $-0.15 \mathrm{~g} / \mathrm{cm}^{3}$ from the nominal value of $1.85 \mathrm{~g} / \mathrm{cm}^{3}$ (Table 1.14 of HTTR-GCR-RESR-001) to determine the effective uncertainty in $\mathrm{k}_{\text {eff. }}$ These values are three times the bounding limit. Results are shown in Table 2.43.

The total number of TRISO particles used in the fully-loaded core is approximately $868,140,000$. For determining the random component of the uncertainty, the results in Table 2.43 would be divided by $\sqrt{\mathrm{N}}$, where $\mathrm{N}$ for each case is shown in Table 2.43 . 
NEA/NSC/DOC(2006)1

\section{Gas Cooled (Thermal) Reactor - GCR \\ HTTR-GCR-RESR-002 \\ CRIT-REAC-RRATE}

Table 2.43. Effect of Uncertainty in IPyC Density.

\begin{tabular}{|c|c|c|c|c|c|c|c|c|c|}
\hline Case & Deviation & $\Delta \mathrm{k}$ & \pm & $\sigma_{\Delta \mathrm{k}}$ & $\begin{array}{l}\text { Scaling } \\
\text { Factor }\end{array}$ & $\Delta \mathrm{k}_{\mathrm{eff}}(1 \sigma)$ & \pm & $\sigma_{\Delta \mathrm{keff}}$ & $\mathrm{N}$ \\
\hline \multirow{2}{*}{1} & -0.15 & 0.00000 & \pm & 0.00016 & $3 \sqrt{3}$ & 0.00000 & \pm & 0.00003 & \multirow{2}{*}{$5.38 \times 10^{8}$} \\
\hline & $+0.30 \mathrm{~g} / \mathrm{cm}^{3}$ & 0.00017 & \pm & 0.00017 & $3 \sqrt{3}$ & 0.00003 & \pm & 0.00003 & \\
\hline \multirow{2}{*}{2} & $-0.15 \mathrm{~g} / \mathrm{cm}^{3}$ & 0.00012 & \pm & 0.00016 & $3 \sqrt{3}$ & 0.00002 & \pm & 0.00003 & \multirow{2}{*}{$5.98 \times 10^{8}$} \\
\hline & $+0.30 \mathrm{~g} / \mathrm{cm}^{3}$ & 0.00027 & - & 0.00 & $3 \sqrt{3}$ & 05 & \pm & 003 & \\
\hline \multirow{2}{*}{3} & $-0.15 \mathrm{~g} / \mathrm{cm}^{3}$ & 0.00006 & \pm & 0.00016 & $3 \sqrt{3}$ & 0.00001 & \pm & 0.00003 & \multirow{2}{*}{$6.88 \times 10^{8}$} \\
\hline & $+0.30 \mathrm{~g} / \mathrm{cm}^{3}$ & 0.00010 & & 0.00017 & $3 \sqrt{3}$ & 0.00002 & \pm & 0.00003 & \\
\hline \multirow{2}{*}{4} & $-0.15 \mathrm{~g} / \mathrm{cm}^{3}$ & -0.00011 & \pm & 0.00017 & $3 \sqrt{3}$ & -0.00002 & \pm & 0.00003 & \multirow{2}{*}{$6.88 \times 10^{8}$} \\
\hline & $+0.30 \mathrm{~g} / \mathrm{cm}^{3}$ & -0.00005 & \pm & 0.00017 & $3 \sqrt{3}$ & -0.00001 & \pm & 0.00003 & \\
\hline \multirow[b]{2}{*}{5} & $-0.15 \mathrm{~g} / \mathrm{cm}^{3}$ & 0.00015 & t & 0.00016 & $3 \sqrt{3}$ & 0.00003 & $\perp$ & 0.00003 & \multirow{2}{*}{$7.78 \times 10^{8}$} \\
\hline & $+0.30 \mathrm{~g} / \mathrm{cm}^{3}$ & 0.00010 & \pm & 0.00016 & $3 \sqrt{3}$ & 0.00002 & \pm & 0.00003 & \\
\hline
\end{tabular}

\section{$\underline{\text { IPyC Impurity }}$}

The IPyC impurity was varied from 0-3 ppm by weight of equivalent natural-boron content to determine the bounding uncertainty in $\mathrm{k}_{\text {eff. }}$ The average value is $1.5 \mathrm{ppm}$ by weight (Table 1.13 of HTTR-GCR-RESR-001). Results are shown in Table 2.28.

The uncertainty in the IPyC impurity is considered all systematic with no random component.

Table 2.28. Effect of Uncertainty in IPyC Impurity.

\begin{tabular}{||c|c|ccc|c|ccc||}
\hline \hline Case & Deviation & $\Delta \mathrm{k}$ & \pm & $\sigma_{\Delta \mathrm{k}}$ & $\begin{array}{c}\text { Scaling } \\
\text { Factor }\end{array}$ & $\Delta \mathrm{k}_{\text {eff }}(1 \sigma)$ & \pm & $\sigma_{\Delta \mathrm{keff}}$ \\
\hline \hline \multirow{2}{*}{1} & $0 \mathrm{ppm}$ & 0.00006 & \pm & 0.00017 & $\sqrt{ } 3$ & 0.00003 & \pm & 0.00010 \\
& $3 \mathrm{ppm}$ & -0.00006 & \pm & 0.00017 & $\sqrt{3}$ & -0.00003 & \pm & 0.00010 \\
\hline \multirow{2}{*}{2} & $0 \mathrm{ppm}$ & 0.00002 & \pm & 0.00016 & $\sqrt{ } 3$ & 0.00001 & \pm & 0.00009 \\
& $3 \mathrm{ppm}$ & 0.00008 & \pm & 0.00017 & $\sqrt{3}$ & 0.00005 & \pm & 0.00010 \\
3 & $0 \mathrm{ppm}$ & -0.00019 & \pm & 0.00016 & $\sqrt{3}$ & -0.00011 & \pm & 0.00009 \\
& $3 \mathrm{ppm}$ & -0.00027 & \pm & 0.00017 & $\sqrt{3}$ & -0.00016 & \pm & 0.00010 \\
\hline \multirow{3}{*}{4} & $0 \mathrm{ppm}$ & 0.00005 & \pm & 0.00017 & $\sqrt{3}$ & 0.00003 & \pm & 0.00010 \\
& $3 \mathrm{ppm}$ & -0.00005 & \pm & 0.00017 & $\sqrt{3}$ & -0.00003 & \pm & 0.00010 \\
\hline \multirow{2}{*}{5} & $0 \mathrm{ppm}$ & 0.00023 & \pm & 0.00016 & $\sqrt{3}$ & 0.00013 & \pm & 0.00009 \\
& $3 \mathrm{ppm}$ & -0.00011 & \pm & 0.00016 & $\sqrt{3}$ & -0.00006 & \pm & 0.00009 \\
\hline \hline
\end{tabular}

\section{SiC Density}

The SiC density was increased by $0.06 \mathrm{~g} / \mathrm{cm}^{3}$, where $0.02 \mathrm{~g} / \mathrm{cm}^{3}$ is typical for $\mathrm{SiC}$ material, from the nominal value of $3.20 \mathrm{~g} / \mathrm{cm}^{3}$ (Table 1.14 of HTTR-GCR-RESR-001) to determine the effective 
NEA/NSC/DOC(2006)1

\section{Gas Cooled (Thermal) Reactor - GCR \\ HTTR-GCR-RESR-002 \\ CRIT-REAC-RRATE}

uncertainty in $\mathrm{k}_{\text {eff. }}$ This value is three times the bounding limit. The density was not decreased because of the minimum requirement for $\mathrm{SiC}$ density. Results are shown in Table 2.44.

The total number of TRISO particles used in the fully-loaded core is approximately $868,140,000$. For determining the random component of the uncertainty, the results in Table 2.44 would be divided by $\sqrt{ } \mathrm{N}$, where $\mathrm{N}$ for each case is shown in Table 2.44 .

Table 2.44. Effect of Uncertainty in SiC Density.

\begin{tabular}{|c|c|ccc|c|ccc|c||}
\hline \hline Case & Deviation & $\Delta \mathrm{k}$ & \pm & $\sigma_{\Delta \mathrm{k}}$ & $\begin{array}{c}\text { Scaling } \\
\text { Factor }\end{array}$ & $\Delta \mathrm{k}_{\text {eff }}(1 \sigma)$ & \pm & $\sigma_{\Delta \mathrm{keff}}$ & $\mathrm{N}$ \\
\hline \hline 1 & $+0.06 \mathrm{~g} / \mathrm{cm}^{3}$ & 0.00003 & \pm & 0.00016 & $6 \sqrt{3}$ & 0.00000 & \pm & 0.00002 & $5.38 \times 10^{8}$ \\
\hline 2 & $+0.06 \mathrm{~g} / \mathrm{cm}^{3}$ & 0.00029 & \pm & 0.00017 & $6 \sqrt{3}$ & 0.00003 & \pm 0.00002 & $5.98 \times 10^{8}$ \\
\hline 3 & $+0.06 \mathrm{~g} / \mathrm{cm}^{3}$ & -0.00014 & \pm & 0.00017 & $6 \sqrt{3}$ & -0.00001 & \pm 0.00002 & $6.88 \times 10^{8}$ \\
\hline 4 & $+0.06 \mathrm{~g} / \mathrm{cm}^{3}$ & -0.00026 & \pm & 0.00017 & $6 \sqrt{3}$ & -0.00003 & \pm 0.00002 & $6.88 \times 10^{8}$ \\
\hline 5 & $+0.06 \mathrm{~g} / \mathrm{cm}^{3}$ & -0.00018 & \pm & 0.00016 & $6 \sqrt{3}$ & -0.00002 & \pm 0.00002 & $7.78 \times 10^{8}$ \\
\hline
\end{tabular}

\section{SiC Impurity}

The $\mathrm{SiC}$ impurity was varied from $0-3 \mathrm{ppm}$ by weight of equivalent natural-boron content to determine the bounding uncertainty in $\mathrm{k}_{\text {eff. }}$. The average value is $1.5 \mathrm{ppm}$ by weight (Table 1.13 of HTTR-GCR-RESR-001). Results are shown in Table 2.45.

The uncertainty in the SiC impurity is considered all systematic with no random component.

Table 2.45. Effect of Uncertainty in SiC Impurity.

\begin{tabular}{||c|c|ccc|c|ccc||}
\hline \hline Case & Deviation & $\Delta \mathrm{k}$ & \pm & $\sigma_{\Delta \mathrm{k}}$ & $\begin{array}{c}\text { Scaling } \\
\text { Factor }\end{array}$ & $\Delta \mathrm{k}_{\text {eff }}(1 \sigma)$ & \pm & $\sigma_{\Delta \mathrm{keff}}$ \\
\hline \hline \multirow{2}{*}{1} & $0 \mathrm{ppm}$ & 0.00007 & \pm & 0.00017 & $\sqrt{ } 3$ & 0.00004 & \pm & 0.00010 \\
& $3 \mathrm{ppm}$ & 0.00001 & \pm & 0.00017 & $\sqrt{ } 3$ & 0.00001 & \pm & 0.00010 \\
\hline \multirow{2}{*}{2} & $0 \mathrm{ppm}$ & 0.00017 & \pm & 0.00016 & $\sqrt{ } 3$ & 0.00010 & \pm & 0.00009 \\
& $3 \mathrm{ppm}$ & -0.00013 & \pm & 0.00016 & $\sqrt{ } 3$ & -0.00008 & \pm & 0.00009 \\
\hline \multirow{3}{*}{3} & $0 \mathrm{ppm}$ & -0.00012 & \pm & 0.00017 & $\sqrt{3}$ & -0.00007 & \pm & 0.00010 \\
& $3 \mathrm{ppm}$ & -0.00014 & \pm & 0.00017 & $\sqrt{3}$ & -0.00008 & \pm & 0.00010 \\
\hline \multirow{3}{*}{4} & $0 \mathrm{ppm}$ & 0.00000 & \pm & 0.00016 & $\sqrt{3}$ & 0.00000 & \pm & 0.00009 \\
& $3 \mathrm{ppm}$ & -0.00019 & \pm & 0.00017 & $\sqrt{3}$ & -0.00011 & \pm & 0.00010 \\
\hline \multirow{2}{*}{5} & $0 \mathrm{ppm}$ & 0.00000 & \pm & 0.00016 & $\sqrt{3}$ & 0.00000 & \pm & 0.00009 \\
& $3 \mathrm{ppm}$ & -0.00027 & \pm & 0.00016 & $\sqrt{3}$ & -0.00016 & \pm & 0.00009 \\
\hline \hline
\end{tabular}


NEA/NSC/DOC(2006)1

Gas Cooled (Thermal) Reactor - GCR

HTTR-GCR-RESR-002

CRIT-REAC-RRATE

\section{OPyC Density}

The OPyC density was varied +0.30 and $-0.15 \mathrm{~g} / \mathrm{cm}^{3}$ from the nominal value of $1.85 \mathrm{~g} / \mathrm{cm}^{3}$ (Table 1.14 of HTTR-GCR-RESR-001) to determine the effective uncertainty in $\mathrm{k}_{\text {eff. }}$ This value is three times the bounding limit. Results are shown in Table 2.46.

The total number of TRISO particles used in the fully-loaded core is approximately $868,140,000$. For determining the random component of the uncertainty, the results in Table 2.46 would be divided by $\sqrt{\mathrm{N}}$, where $\mathrm{N}$ for each case is shown in Table 2.46.

Table 2.46. Effect of Uncertainty in OPyC Density.

\begin{tabular}{|c|c|c|c|c|c|c|c|c|c|}
\hline Case & Deviation & $\Delta \mathrm{k}$ & \pm & $\sigma_{\Delta \mathrm{k}}$ & $\begin{array}{c}\text { Scaling } \\
\text { Factor }\end{array}$ & $\Delta \mathrm{k}_{\mathrm{eff}}(1 \sigma)$ & \pm & $\sigma_{\Delta \mathrm{keff}}$ & $\mathrm{N}$ \\
\hline & $-0.15 \mathrm{~g} / \mathrm{cm}^{3}$ & -0.00012 & \pm & 0.00017 & $3 \sqrt{3}$ & -0.00002 & \pm & 0.00003 & \multirow{2}{*}{$5.38 \times 10^{8}$} \\
\hline & $+0.30 \mathrm{~g} / \mathrm{cm}^{3}$ & 009 & \pm & 17 & $3 \sqrt{3}$ & 02 & \pm & 003 & \\
\hline \multirow{2}{*}{2} & $-0.15 \mathrm{~g} / \mathrm{cm}^{3}$ & 0.00005 & \pm & 0.00017 & $3 \sqrt{3}$ & 0.00001 & \pm & 0.00003 & \multirow{2}{*}{$5.98 \times 10^{8}$} \\
\hline & $+0.30 \mathrm{~g} / \mathrm{cm}^{3}$ & 0.0 & \pm & 16 & $3 \sqrt{3}$ & 05 & \pm & 003 & \\
\hline \multirow{2}{*}{3} & $-0.15 \mathrm{~g} / \mathrm{cm}^{3}$ & -0.00018 & \pm & 0.00016 & $3 \sqrt{3}$ & -0.00003 & \pm & 0.00003 & \multirow{2}{*}{$6.88 \times 10^{8}$} \\
\hline & $+0.30 \mathrm{~g} / \mathrm{cm}^{3}$ & -0.00026 & \pm & 0.00016 & $3 \sqrt{3}$ & -0.00005 & $\perp$ & 0.00003 & \\
\hline \multirow{2}{*}{4} & $-0.15 \mathrm{~g} / \mathrm{cm}^{3}$ & -0.00012 & \pm & 0.00017 & $3 \sqrt{3}$ & -0.00011 & \pm & 0.00010 & \multirow{2}{*}{$6.88 \times 10^{8}$} \\
\hline & $+0.30 \mathrm{~g} / \mathrm{cm}^{3}$ & -0.00004 & \pm & 0.00017 & $3 \sqrt{3}$ & -0.00001 & \pm & 0.00003 & \\
\hline \multirow{2}{*}{5} & $-0.15 \mathrm{~g} / \mathrm{cm}^{3}$ & -0.00010 & \pm & 0.00016 & $3 \sqrt{3}$ & -0.00002 & 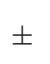 & 0.00003 & \multirow{2}{*}{$7.78 \times 10^{8}$} \\
\hline & $+0.30 \mathrm{~g} / \mathrm{cm}^{3}$ & 0.00009 & \pm & 0.00016 & $3 \sqrt{3}$ & 0.00002 & \pm & 0.00003 & \\
\hline
\end{tabular}

\section{OPyC Impurity}

The OPyC impurity was varied from 0-3 ppm by weight of equivalent natural-boron content to determine the bounding uncertainty in $\mathrm{k}_{\text {eff. }}$ The average value is $1.5 \mathrm{ppm}$ by weight (Table 1.13 of HTTR-GCR-RESR-001). Results are shown in Table 2.47.

The uncertainty in the OPyC impurity is considered all systematic with no random component. 
NEA/NSC/DOC(2006)1

Gas Cooled (Thermal) Reactor - GCR

HTTR-GCR-RESR-002

CRIT-REAC-RRATE

Table 2.47. Effect of Uncertainty in OPyC Impurity.

\begin{tabular}{||c|c|ccc|c|ccc||}
\hline \hline Case & Deviation & $\Delta \mathrm{k}$ & \pm & $\sigma_{\Delta \mathrm{k}}$ & $\begin{array}{c}\text { Scaling } \\
\text { Factor }\end{array}$ & $\Delta \mathrm{k}_{\text {eff }}(1 \sigma)$ & \pm & $\sigma_{\Delta \mathrm{keff}}$ \\
\hline \hline \multirow{2}{*}{1} & $0 \mathrm{ppm}$ & 0.00009 & \pm & 0.00017 & $\sqrt{ } 3$ & 0.00005 & \pm & 0.00010 \\
& $3 \mathrm{ppm}$ & 0.00009 & \pm & 0.00017 & $\sqrt{ } 3$ & 0.00005 & \pm & 0.00010 \\
\hline \multirow{2}{*}{2} & $0 \mathrm{ppm}$ & 0.00041 & \pm & 0.00016 & $\sqrt{ } 3$ & 0.00024 & \pm & 0.00009 \\
& $3 \mathrm{ppm}$ & -0.00026 & \pm & 0.00016 & $\sqrt{ } 3$ & -0.00015 & \pm & 0.00009 \\
\hline \multirow{3}{*}{3} & $0 \mathrm{ppm}$ & -0.00018 & \pm & 0.00016 & $\sqrt{3}$ & -0.00003 & \pm & 0.00003 \\
& $3 \mathrm{ppm}$ & -0.00026 & \pm & 0.00016 & $\sqrt{3}$ & -0.00005 & \pm & 0.00003 \\
4 & $0 \mathrm{ppm}$ & -0.00020 & \pm & 0.00017 & $\sqrt{3}$ & -0.00012 & \pm & 0.00010 \\
& $3 \mathrm{ppm}$ & -0.00005 & \pm & 0.00017 & $\sqrt{3}$ & -0.00003 & \pm & 0.00010 \\
\hline \multirow{2}{*}{5} & $0 \mathrm{ppm}$ & 0.00034 & \pm & 0.00016 & $\sqrt{3}$ & 0.000020 & \pm & 0.00009 \\
& $3 \mathrm{ppm}$ & -0.00040 & \pm & 0.00016 & $\sqrt{3}$ & -0.00023 & \pm & 0.00009 \\
\hline
\end{tabular}

\section{Overcoat Density}

Because insufficient data is available for the final composition and density of the graphite overcoat, this layer is being treated with equal properties to that of the surrounding compact graphite matrix. The overcoat density was varied $\pm 0.15 \mathrm{~g} / \mathrm{cm}^{3}$ to determine the effective uncertainty in $\mathrm{k}_{\mathrm{eff}}$. This value is three times the bounding limit. Results are shown in Table 2.48.

The total number of TRISO particles used in the fully-loaded core is approximately $868,140,000$. For determining the random component of the uncertainty, the results in Table 2.48 would be divided by $\sqrt{ } \mathrm{N}$, where $\mathrm{N}$ for each case is shown in Table 2.48 .

Table 2.48. Effect of Uncertainty in Overcoat Density.

\begin{tabular}{|c|c|c|c|c|c|c|c|c|c|}
\hline Case & Deviation & $\Delta \mathrm{k}$ & & $\sigma_{\Delta \mathrm{k}}$ & $\begin{array}{l}\text { Scaling } \\
\text { Factor }\end{array}$ & $\Delta \mathrm{k}_{\mathrm{eff}}(1 \sigma)$ & \pm & $\sigma_{\Delta \mathrm{keff}}$ & $\mathrm{N}$ \\
\hline & $-0.15 \mathrm{~g} / \mathrm{cm}^{3}$ & -0.0 & & 0.00017 & $3 \sqrt{3}$ & -0.00006 & \pm & 0.00003 & \multirow{2}{*}{$5.38 \times 10^{8}$} \\
\hline & $+0.15 \mathrm{~g} / \mathrm{cm}^{3}$ & 0.00034 & & 0.00017 & $3 \sqrt{3}$ & 0.00007 & \pm & 0.00003 & \\
\hline \multirow{2}{*}{2} & $-0.15 \mathrm{~g} / \mathrm{cm}^{3}$ & -0.00018 & & 0.00017 & $3 \sqrt{3}$ & -0.00003 & \pm & 0.00003 & \multirow{2}{*}{$5.98 \times 10^{8}$} \\
\hline & $+0.15 \mathrm{~g} / \mathrm{cm}^{3}$ & 0. & & 6 & $3 \sqrt{3}$ & 8 & \pm & 03 & \\
\hline \multirow[b]{2}{*}{3} & $-0.15 \mathrm{~g} / \mathrm{cm}^{3}$ & -0.00050 & & 0.00016 & $3 \sqrt{3}$ & -0.00010 & \pm & 0.00003 & \multirow{2}{*}{$6.88 \times 10^{8}$} \\
\hline & $+0.15 \mathrm{~g} / \mathrm{cm}^{3}$ & 0.00014 & & 00017 & $3 \sqrt{3}$ & 0.00003 & \pm & 03 & \\
\hline \multirow{2}{*}{4} & $-0.15 \mathrm{~g} / \mathrm{cm}^{3}$ & -0.00058 & & 0.00016 & $3 \sqrt{3}$ & -0.00011 & \pm & 0.00003 & \multirow{2}{*}{$6.88 \times 10^{8}$} \\
\hline & $+0.15 \mathrm{~g} / \mathrm{cm}^{3}$ & 0.00032 & & 0.00017 & $3 \sqrt{3}$ & 0.00006 & \pm & .00003 & \\
\hline \multirow{2}{*}{5} & $-0.15 \mathrm{~g} / \mathrm{cm}^{3}$ & -0.00024 & & 0.00016 & $3 \sqrt{3}$ & -0.00005 & \pm & 0.00003 & \multirow{2}{*}{$7.78 \times 10^{8}$} \\
\hline & $+0.15 \mathrm{~g} / \mathrm{cm}^{3}$ & 0.00025 & & 0.00016 & $3 \sqrt{3}$ & 0.00005 & \pm & 0.00003 & \\
\hline
\end{tabular}


NEA/NSC/DOC(2006)1

\section{Gas Cooled (Thermal) Reactor - GCR \\ HTTR-GCR-RESR-002 \\ CRIT-REAC-RRATE}

\section{Overcoat Composition}

Because insufficient data is available for the final composition and density of the graphite overcoat, this layer is being treated with equal properties to that of the surrounding compact graphite matrix. In essence, the uncertainty has already been accounted for, as the surrounding graphite matrix has been characterized for uncertainties, and the overcoat has been demonstrated elsewhere to have negligible impact on the calculation of $k_{\text {eff }}$ when it is not explicitly modeled, but included in the surrounding matrix. ${ }^{a}$

\section{Overcoat Impurity}

The overcoat impurity was varied from $0-5 \mathrm{ppm}$ by weight of equivalent natural-boron content to determine the bounding uncertainty in $\mathrm{k}_{\text {eff. }}$. The average value of the coated fuel particles is $1.5 \mathrm{ppm}$ by weight (Table 1.13 of HTTR-GCR-RESR-001); it is assumed that the overcoat would have a comparable impurity amount. The alternative is to use the impurity of the compact (matrix) material, which is 0.82 ppm by weight (Table 1.27 of HTTR-GCR-RESR-001). The larger amount is selected, i.e. $1.5 \mathrm{ppm}$. Results are shown in Table 2.49.

The uncertainty in the overcoat impurity is considered all systematic with no random component.

Table 2.49. Effect of Uncertainty in Overcoat Impurity.

\begin{tabular}{||c|c|ccc|c|ccc||}
\hline \hline Case & Deviation & $\Delta \mathrm{k}$ & \pm & $\sigma_{\Delta \mathrm{k}}$ & $\begin{array}{c}\text { Scaling } \\
\text { Factor }\end{array}$ & $\Delta \mathrm{k}_{\text {eff }}(1 \sigma)$ & \pm & $\sigma_{\Delta \mathrm{keff}}$ \\
\hline \hline \multirow{2}{*}{1} & $0 \mathrm{ppm}$ & 0.00046 & \pm & 0.00017 & $\sqrt{ } 3$ & 0.00027 & \pm & 0.00010 \\
& $5 \mathrm{ppm}$ & -0.00069 & \pm & 0.00017 & $\sqrt{ } 3$ & -0.00040 & \pm & 0.00010 \\
\hline \multirow{2}{*}{2} & $0 \mathrm{ppm}$ & 0.00052 & \pm & 0.00017 & $\sqrt{ } 3$ & 0.00030 & \pm & 0.00010 \\
& $5 \mathrm{ppm}$ & -0.00103 & \pm & 0.00017 & $\sqrt{3}$ & -0.00059 & \pm & 0.00010 \\
\hline \multirow{3}{*}{3} & $0 \mathrm{ppm}$ & 0.00077 & \pm & 0.00016 & $\sqrt{ } 3$ & 0.00044 & \pm & 0.00009 \\
& $5 \mathrm{ppm}$ & -0.00122 & \pm & 0.00017 & $\sqrt{3}$ & -0.00070 & \pm & 0.00010 \\
4 & $0 \mathrm{ppm}$ & 0.00044 & \pm & 0.00017 & $\sqrt{3}$ & 0.00025 & \pm & 0.00010 \\
& $5 \mathrm{ppm}$ & -0.00132 & \pm & 0.00017 & $\sqrt{ } 3$ & -0.00076 & \pm & 0.00010 \\
\hline \multirow{2}{*}{5} & $0 \mathrm{ppm}$ & 0.00055 & \pm & 0.00016 & $\sqrt{3}$ & 0.00032 & \pm & 0.00009 \\
& $5 \mathrm{ppm}$ & -0.00119 & \pm & 0.00016 & $\sqrt{3}$ & -0.00069 & \pm & 0.00009 \\
\hline
\end{tabular}

\subsubsection{Prismatic Fuel Compact}

\section{Density}

The compact matrix density was varied $\pm 0.15 \mathrm{~g} / \mathrm{cm}^{3}$ from the nominal value of $1.70 \mathrm{~g} / \mathrm{cm}^{3}$ (Table $1.14 \mathrm{of}$ HTTR-GCR-RESR-001) to determine the effective uncertainty in $\mathrm{k}_{\text {eff. }}$ This value is three times the bounding limit. Results are shown in Table 2.50.

\footnotetext{
${ }^{a}$ W. Ji, J. L. Conlin, W. R. Martin, J. C. Lee, and F. B. Brown, "Explicit Modeling of Particle Fuel for the VeryHigh Temperature Gas-Cooled Reactor,” Trans. Am. Nucl. Soc., 92 (June 2005).
} 
NEA/NSC/DOC(2006)1

\section{Gas Cooled (Thermal) Reactor - GCR \\ HTTR-GCR-RESR-002 \\ CRIT-REAC-RRATE}

The total number of fuel compacts used in the fully-loaded core is 66,780. For determining the random component of the uncertainty, the results in Table 2.50 would be divided by $\sqrt{ } \mathrm{N}$, where $\mathrm{N}$ for each case is shown in Table 2.50 .

Table 2.50. Effect of Uncertainty in Compact Matrix Density.

\begin{tabular}{|c|c|c|c|c|c|c|c|c|c|}
\hline Case & Deviation & $\Delta \mathrm{k}$ & \pm & $\sigma_{\Delta \mathrm{k}}$ & $\begin{array}{l}\text { Scaling } \\
\text { Factor }\end{array}$ & $\Delta \mathrm{k}_{\mathrm{eff}}(1 \sigma)$ & \pm & $\sigma_{\Delta \mathrm{keff}}$ & $\mathrm{N}$ \\
\hline \multirow{2}{*}{1} & $-0.15 \mathrm{~g} / \mathrm{cm}^{3}$ & -0.00009 & \pm & 0.00016 & $3 \sqrt{3}$ & -0.00002 & \pm & 0.00003 & \multirow{2}{*}{41,370} \\
\hline & $+0.15 \mathrm{~g} / \mathrm{cm}^{3}$ & -0.00012 & \pm & 0.00017 & $3 \sqrt{3}$ & -0.00002 & \pm & 0.00003 & \\
\hline \multirow{2}{*}{2} & $-0.15 \mathrm{~g} / \mathrm{cm}^{3}$ & 0.00016 & \pm & 0.00017 & $3 \sqrt{3}$ & 0.00003 & \pm & 0.00003 & \multirow{2}{*}{45,990} \\
\hline & $+0.15 \mathrm{~g} / \mathrm{cm}^{3}$ & 0.00016 & \pm & 0.00016 & $3 \sqrt{3}$ & 0.00003 & \pm & 0.00003 & \\
\hline \multirow{2}{*}{3} & $-0.15 \mathrm{~g} / \mathrm{cm}^{3}$ & -0.00023 & \pm & 0.00016 & $3 \sqrt{3}$ & -0.00004 & \pm & 0.00003 & \multirow{2}{*}{52,920} \\
\hline & $+0.15 \mathrm{~g} / \mathrm{cm}^{3}$ & 0.00000 & \pm & 0.00016 & $3 \sqrt{3}$ & 0.00000 & \pm & 0.00003 & \\
\hline \multirow{2}{*}{4} & $-0.15 \mathrm{~g} / \mathrm{cm}^{3}$ & -0.00028 & \pm & 0.00017 & $3 \sqrt{3}$ & 0.00005 & \pm & 0.00003 & \multirow{2}{*}{52,920} \\
\hline & $+0.15 \mathrm{~g} / \mathrm{cm}^{3}$ & 0.00022 & \pm & 0.00017 & $3 \sqrt{3}$ & 0.00004 & \pm & 0.00003 & \\
\hline \multirow{2}{*}{5} & $-0.15 \mathrm{~g} / \mathrm{cm}^{3}$ & -0.00011 & \pm & 0.00016 & $3 \sqrt{3}$ & -0.00002 & \pm & 0.00003 & \multirow{2}{*}{59,850} \\
\hline & $+0.15 \mathrm{~g} / \mathrm{cm}^{3}$ & 0.00028 & \pm & 0.00016 & $3 \sqrt{3}$ & 0.00005 & \pm & 0.00003 & \\
\hline
\end{tabular}

\section{Impurity}

The compact matrix impurity was varied from $0-5 \mathrm{ppm}$ by weight of equivalent natural-boron content (Table 1.14 of HTTR-GCR-RESR-001) to determine the bounding uncertainty in $\mathrm{k}_{\mathrm{eff}}$. The nominal impurity is $0.82 \mathrm{ppm}$ of natural boron by weight (Table 1.27 of HTTR-GCR-RESR-001). Results are shown in Table 2.51 .

The uncertainty in the fuel compact impurity is considered all systematic with no random component.

Table 2.51. Effect of Uncertainty in Compact Matrix Impurity.

\begin{tabular}{||c|c|ccc|c|ccc||}
\hline \hline Case & Deviation & $\Delta \mathrm{k}$ & \pm & $\sigma_{\Delta \mathrm{k}}$ & $\begin{array}{c}\text { Scaling } \\
\text { Factor }\end{array}$ & $\Delta \mathrm{k}_{\text {eff }}(1 \sigma)$ & \pm & $\sigma_{\Delta \mathrm{keff}}$ \\
\hline \hline \multirow{2}{*}{1} & $0 \mathrm{ppm}$ & 0.00007 & \pm & 0.00016 & $\sqrt{ } 3$ & 0.00004 & \pm & 0.00009 \\
& $5 \mathrm{ppm}$ & -0.00074 & \pm & 0.00017 & $\sqrt{3}$ & -0.00043 & \pm & 0.00010 \\
2 & $0 \mathrm{ppm}$ & 0.00023 & \pm & 0.00016 & $\sqrt{ } 3$ & 0.00013 & \pm & 0.00009 \\
& $5 \mathrm{ppm}$ & -0.00072 & \pm & 0.00016 & $\sqrt{3}$ & -0.00042 & \pm & 0.00009 \\
\hline \multirow{2}{*}{3} & $0 \mathrm{ppm}$ & 0.00008 & \pm & 0.00017 & $\sqrt{3}$ & 0.00005 & \pm & 0.00010 \\
& $5 \mathrm{ppm}$ & -0.00115 & \pm & 0.00016 & $\sqrt{3}$ & -0.00066 & \pm & 0.00009 \\
\hline \multirow{3}{*}{4} & $0 \mathrm{ppm}$ & 0.00014 & \pm & 0.00017 & $\sqrt{3}$ & 0.00008 & \pm & 0.00010 \\
& $5 \mathrm{ppm}$ & -0.00104 & \pm & 0.00017 & $\sqrt{3}$ & -0.00060 & \pm & 0.00010 \\
\hline \multirow{2}{*}{5} & $0 \mathrm{ppm}$ & 0.00003 & \pm & 0.00016 & $\sqrt{3}$ & 0.000002 & \pm & 0.00009 \\
& $5 \mathrm{ppm}$ & -0.00113 & \pm & 0.00016 & $\sqrt{3}$ & -0.00065 & \pm & 0.00009 \\
\hline
\end{tabular}

Revision: 0 
NEA/NSC/DOC(2006)1

\section{Gas Cooled (Thermal) Reactor - GCR \\ HTTR-GCR-RESR-002 \\ CRIT-REAC-RRATE}

\section{Free Uranium Content}

The free uranium fraction in the fuel compacts has a maximum bounding limit of 0.000150 (see Table 1.14 of HTTR-GCR-RESR-001). The bounding limit was multiplied by 10 in order to assess the uncertainty in $\mathrm{k}_{\text {eff }}$, but the effects were still negligible. The free uranium was assumed to be $100 \%{ }^{235} \mathrm{U}$ so as to assess the maximum uncertainty. The free uranium content in the graphite compact was not included in the benchmark model and a bias was not applied because the effect was negligible. Results are shown in Table 2.52 .

The uncertainty in the free uranium content is considered all systematic with no random component.

Table 2.52. Effect of Uncertainty in Compact Free Uranium Content.

\begin{tabular}{||c|c|ccc|c|ccc||}
\hline Case & Deviation & $\Delta \mathrm{k}$ & \pm & $\sigma_{\Delta \mathrm{k}}$ & $\begin{array}{c}\text { Scaling } \\
\text { Factor }\end{array}$ & $\Delta \mathrm{k}_{\text {eff }}(1 \sigma)$ & \pm & $\sigma_{\Delta \mathrm{keff}}$ \\
\hline \hline 1 & $1500 \mathrm{ppm}$ & -0.00003 & \pm & 0.00016 & $20 \sqrt{3}$ & 0.00000 & \pm & 0.00000 \\
2 & $1500 \mathrm{ppm}$ & -0.00024 & \pm & 0.00016 & $20 \sqrt{ } 3$ & -0.00001 & \pm & 0.00000 \\
3 & $1500 \mathrm{ppm}$ & -0.00012 & \pm & 0.00017 & $20 \sqrt{3}$ & 0.00000 & \pm & 0.00000 \\
4 & $1500 \mathrm{ppm}$ & 0.00009 & \pm & 0.00017 & $20 \sqrt{3}$ & 0.00000 & \pm & 0.00000 \\
5 & $1500 \mathrm{ppm}$ & 0.00020 & \pm & 0.00016 & $20 \sqrt{3}$ & 0.00001 & \pm & 0.00000 \\
\hline
\end{tabular}

\subsubsection{Graphite Sleeves}

\section{Density}

The graphite sleeve density was varied an assumed $\pm 0.03 \mathrm{~g} / \mathrm{cm}^{3}$ from the nominal value of $1.770 \mathrm{~g} / \mathrm{cm}^{3}$ (Table 1.27 of HTTR-GCR-RESR-001) to determine the effective uncertainty in $\mathrm{k}_{\text {eff. }}$. This value is the bounding limit. The graphite sleeves are composed of IG-110 graphite, and the assumed uncertainty of $0.03 \mathrm{~g} / \mathrm{cm}^{3}$ encompasses the range of reported densities for IG-110 graphite throughout Section 1. Results are shown in Table 2.53.

The total number of graphite sleeves used in the fully-loaded core is 4,770. For determining the random component of the uncertainty, the results in Table 2.53 would be divided by $\sqrt{\mathrm{N}}$, where $\mathrm{N}$ for each case is shown in Table 2.53 . 
NEA/NSC/DOC(2006)1

Gas Cooled (Thermal) Reactor - GCR

HTTR-GCR-RESR-002

CRIT-REAC-RRATE

Table 2.53. Effect of Uncertainty in Graphite Sleeve Density.

\begin{tabular}{|c|c|c|c|c|c|c|c|c|c|}
\hline Case & Deviation & $\Delta \mathrm{k}$ & \pm & $\sigma_{\Delta \mathrm{k}}$ & $\begin{array}{c}\text { Scaling } \\
\text { Factor }\end{array}$ & $\Delta \mathrm{k}_{\mathrm{eff}}(1 \sigma)$ & \pm & $\sigma_{\Delta \mathrm{keff}}$ & $\mathrm{N}$ \\
\hline & $-0.03 \mathrm{~g} / \mathrm{cm}^{3}$ & -0.00036 & & 0.00017 & $\sqrt{3}$ & -0.00021 & \pm & 0.00010 & \multirow{2}{*}{2,955} \\
\hline & $+0.03 \mathrm{~g} / \mathrm{cm}^{3}$ & 0.00030 & & 0.00017 & $\sqrt{3}$ & 0.00017 & \pm & 0.00010 & \\
\hline \multirow[b]{2}{*}{2} & $-0.03 \mathrm{~g} / \mathrm{cm}^{3}$ & -0.00024 & \pm & 0.00016 & $\sqrt{3}$ & -0.00014 & \pm & 0.00009 & \multirow{2}{*}{3,285} \\
\hline & $+0.03 \mathrm{~g} / \mathrm{cm}^{3}$ & 0.00022 & & 0.00017 & $\sqrt{3}$ & 0.00013 & \pm & 0.00010 & \\
\hline \multirow[b]{2}{*}{3} & $-0.03 \mathrm{~g} / \mathrm{cm}^{3}$ & -0.00052 & & 0.00016 & $\sqrt{3}$ & -0.00030 & \pm & 0.00009 & \multirow{2}{*}{3,780} \\
\hline & $+0.03 \mathrm{~g} / \mathrm{cm}^{3}$ & 0.00026 & & 0.00017 & $\sqrt{3}$ & 0.00015 & \pm & 0.00010 & \\
\hline & $-0.03 \mathrm{~g} / \mathrm{cm}^{3}$ & -0.00086 & 工 & 0.00017 & $\sqrt{3}$ & -0.00050 & \pm & 0.00010 & \multirow{2}{*}{3,780} \\
\hline & $+0.03 \mathrm{~g} / \mathrm{cm}^{3}$ & 0.00042 & & 0.00017 & $\sqrt{3}$ & 0.00024 & \pm & 0.00010 & \\
\hline & $-0.03 \mathrm{~g} / \mathrm{cm}^{3}$ & -0.00038 & & 0.00016 & $\sqrt{3}$ & -0.00022 & \pm & 0.00009 & \multirow{2}{*}{4,275} \\
\hline & $+0.03 \mathrm{~g} / \mathrm{cm}^{3}$ & 0.00030 & \pm & 0.00016 & $\sqrt{3}$ & 0.00017 & \pm & 0.00009 & \\
\hline
\end{tabular}

\section{Impurity}

The graphite sleeve impurity was varied from $0-1 \mathrm{ppm}$ by weight of equivalent natural-boron content (Table 1.13 of HTTR-GCR-RESR-001) to determine the bounding uncertainty in $\mathrm{k}_{\text {eff }}$. The nominal impurity is $0.37 \mathrm{ppm}$ of natural boron by weight (Table 1.27 of HTTR-GCR-RESR-001). Results are shown in Table 2.54 .

The uncertainty in the graphite sleeve impurity is considered all systematic with no random component.

Table 2.54. Effect of Uncertainty in Graphite Sleeve Impurity.

\begin{tabular}{||c|c|ccc|c|ccc||}
\hline Case & Deviation & $\Delta \mathrm{k}$ & \pm & $\sigma_{\Delta \mathrm{k}}$ & $\begin{array}{c}\text { Scaling } \\
\text { Factor }\end{array}$ & $\Delta \mathrm{k}_{\text {eff }}(1 \sigma)$ & \pm & $\sigma_{\Delta \mathrm{keff}}$ \\
\hline \hline \multirow{2}{*}{1} & $0 \mathrm{ppm}$ & 0.00007 & \pm & 0.00017 & $\sqrt{3}$ & 0.00004 & \pm & 0.00010 \\
& $1 \mathrm{ppm}$ & -0.00055 & \pm & 0.00017 & $\sqrt{ } 3$ & -0.00032 & \pm & 0.00010 \\
\hline \multirow{3}{*}{2} & $0 \mathrm{ppm}$ & 0.00032 & \pm & 0.00016 & $\sqrt{3}$ & 0.00018 & \pm & 0.00009 \\
& $1 \mathrm{ppm}$ & -0.00037 & \pm & 0.00017 & $\sqrt{3}$ & -0.00021 & \pm & 0.00010 \\
\hline \multirow{3}{*}{3} & $0 \mathrm{ppm}$ & 0.00011 & \pm & 0.00017 & $\sqrt{3}$ & 0.00006 & \pm & 0.00010 \\
& $1 \mathrm{ppm}$ & -0.00084 & \pm & 0.00017 & $\sqrt{3}$ & -0.00048 & \pm & 0.00010 \\
\hline \multirow{3}{*}{4} & $0 \mathrm{ppm}$ & 0.00012 & \pm & 0.00016 & $\sqrt{3}$ & 0.00007 & \pm & 0.00009 \\
& $1 \mathrm{ppm}$ & -0.00058 & \pm & 0.00017 & $\sqrt{3}$ & -0.00033 & \pm & 0.00010 \\
\hline \multirow{3}{*}{5} & $0 \mathrm{ppm}$ & 0.00054 & \pm & 0.00016 & $\sqrt{3}$ & 0.00031 & \pm & 0.00009 \\
& $1 \mathrm{ppm}$ & -0.00053 & \pm & 0.00016 & $\sqrt{3}$ & -0.00031 & \pm & 0.00009 \\
\hline
\end{tabular}

Revision: 0 
NEA/NSC/DOC(2006)1

Gas Cooled (Thermal) Reactor - GCR

HTTR-GCR-RESR-002

CRIT-REAC-RRATE

\subsubsection{Burnable Poisons}

\section{Absorber Density}

The absorber density was varied $\pm 0.03 \mathrm{~g} / \mathrm{cm}^{3}$ from the average value of $1.8 \mathrm{~g} / \mathrm{cm}^{3}$ to determine the effective uncertainty in $\mathrm{k}_{\text {eff }}$. This value is the bounding limit. The density uncertainty is based upon uncertainty typically found in sintered $\mathrm{B}_{4} \mathrm{C} / \mathrm{C}$ pellets (Table 1.29 of HTTR-GCR-RESR-001). Results are shown in Table 2.55 .

The total number of burnable poison pellets used in the fully-loaded core is 5,520. For determining the random component of the uncertainty, the results in Table 2.55 would be divided by $\sqrt{ } \mathrm{N}$, where $\mathrm{N}$ for each case is shown in Table 2.55.

Table 2.55. Effect of Uncertainty in BP Absorber Density.

\begin{tabular}{|c|c|c|c|c|c|c|c|c|c|}
\hline Case & Deviation & $\Delta \mathrm{k}$ & \pm & $\sigma_{\Delta \mathrm{k}}$ & $\begin{array}{c}\text { Scaling } \\
\text { Factor }\end{array}$ & $\Delta \mathrm{k}_{\mathrm{eff}}(1 \sigma)$ & \pm & $\sigma_{\Delta \mathrm{keff}}$ & $\mathrm{N}$ \\
\hline & $-0.03 \mathrm{~g} / \mathrm{cm}^{3}$ & 0.00085 & \pm & 0.00016 & $\sqrt{3}$ & 0.00049 & \pm & 0.00009 & \multirow{2}{*}{3,496} \\
\hline & $+0.03 \mathrm{~g} / \mathrm{cm}^{3}$ & -0.00083 & \pm & 0.00017 & $\sqrt{3}$ & -0.00048 & \pm & 0.00010 & \\
\hline \multirow{2}{*}{2} & $-0.03 \mathrm{~g} / \mathrm{cm}^{3}$ & 0.00101 & \pm & 0.00017 & $\sqrt{3}$ & 0.00058 & \pm & 0.00010 & \multirow{2}{*}{3,864} \\
\hline & $+0.03 \mathrm{~g} / \mathrm{cm}^{3}$ & -0.00048 & 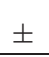 & 0.0 & $\sqrt{3}$ & -0.00028 & \pm & 010 & \\
\hline \multirow[b]{2}{*}{3} & $-0.03 \mathrm{~g} / \mathrm{cm}^{3}$ & 0.00066 & \pm & 0.00017 & $\sqrt{3}$ & 0.00038 & \pm & 0.00010 & \multirow{2}{*}{4,416} \\
\hline & $+0.03 \mathrm{~g} / \mathrm{cm}^{3}$ & -0.00081 & 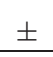 & 0.00017 & $\sqrt{3}$ & -0.00047 & \pm & 0.00010 & \\
\hline \multirow{2}{*}{4} & $-0.03 \mathrm{~g} / \mathrm{cm}^{3}$ & 0.00064 & \pm & 0.00017 & $\sqrt{3}$ & 0.00037 & \pm & 0.00010 & \multirow{2}{*}{4,416} \\
\hline & $+0.03 \mathrm{~g} / \mathrm{cm}^{3}$ & -0.00084 & 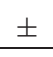 & 0.00016 & $\sqrt{3}$ & -0.00048 & \pm & 0.00009 & \\
\hline & $-0.03 \mathrm{~g} / \mathrm{cm}^{3}$ & 0.00065 & \pm & 0.00016 & $\sqrt{3}$ & 0.00038 & \pm & 0.00009 & \multirow{2}{*}{4,968} \\
\hline & $+0.03 \mathrm{~g} / \mathrm{cm}^{3}$ & -0.00077 & \pm & 0.00016 & $\sqrt{3}$ & -0.00044 & \pm & 0.00009 & \\
\hline
\end{tabular}

\section{Absorber Content}

The uncertainty in the absorber content was not provided and the variation provided in Table 1.29 of HTTR-GCR-RESR-001 appears too excessive for the quantities utilized in the BP pellets. A variation of approximately $\pm 0.25 \%$ by weight was assumed for each of the two absorber pellet types, and the effective uncertainty in $\mathrm{k}_{\text {eff }}$ was determined. This value is treated as a bounding limit. The uncertainty of $\pm 0.25 \%$ is based upon the assumption that burnable poison pellets with boron contents between 1.75 and 2.25 wt.\% would have an average content of $2.00 \mathrm{wt} . \%$ and boron contents between 2.25 and 2.75 wt.\% would have an average content of $2.50 \mathrm{wt} . \%$. Further information would be necessary to reduce the range of the uncertainty. Results are shown in Tables 2.56 and 2.57 .

The total number of burnable poison pellets with weight percents of 2.00 and 2.50 used in the fullyloaded core is 3,600 and 1,920, respectively. For determining the random component of the uncertainty, the results in Tables 2.56 and 2.57 would be divided by their respective $\sqrt{ } \mathrm{N}$ value, where $\mathrm{N}$ for each case is shown in Tables 2.56 and 2.57. 
NEA/NSC/DOC(2006)1

Gas Cooled (Thermal) Reactor - GCR

HTTR-GCR-RESR-002

CRIT-REAC-RRATE

Table 2.56. Effect of Uncertainty in BP Absorber Content (nominal 2.00 wt.\%).

\begin{tabular}{|c|c|c|c|c|c|c|c|c|c|}
\hline Case & Deviation & $\Delta \mathrm{k}$ & \pm & $\sigma_{\Delta \mathrm{k}}$ & $\begin{array}{l}\text { Scaling } \\
\text { Factor }\end{array}$ & $\Delta \mathrm{k}_{\mathrm{eff}}(1 \sigma)$ & \pm & $\sigma_{\Delta \mathrm{keff}}$ & $\mathrm{N}$ \\
\hline & -0.25 wt. $\%$ & 0.00265 & & 0.00016 & $\sqrt{3}$ & 0.000153 & & 0.00009 & \multirow{2}{*}{2,280} \\
\hline & +0.25 wt. $\%$ & -0.00234 & \pm & 0.00017 & $\sqrt{3}$ & -0.00135 & \pm & 0.00010 & \\
\hline \multirow{2}{*}{2} & -0.25 wt. $\%$ & 0.00304 & \pm & 0.00017 & $\sqrt{3}$ & 0.00176 & + & 0.00010 & \multirow{2}{*}{2,520} \\
\hline & +0.25 wt. $\%$ & -0.00234 & & 0.00016 & $\sqrt{3}$ & -0.00135 & & 0009 & \\
\hline \multirow{2}{*}{3} & -0.25 wt. $\%$ & 0.00394 & \pm & 0.00017 & $\sqrt{3}$ & 0.00227 & & 0.00010 & \multirow{2}{*}{2,880} \\
\hline & +0.25 wt. $\%$ & -0.00342 & & 0.00017 & $\sqrt{3}$ & -0.00197 & & 0.00010 & \\
\hline \multirow{2}{*}{4} & -0.25 wt. $\%$ & 0.00379 & & 0.00016 & $\sqrt{3}$ & -0.00219 & & 0.00009 & \multirow{2}{*}{2,880} \\
\hline & +0.25 wt. $\%$ & -0.00346 & & 0.00017 & $\sqrt{3}$ & -0.00200 & & 0.00010 & \\
\hline & -0.25 wt. $\%$ & 0.00518 & & 0.00016 & $\sqrt{3}$ & 0.00299 & & 0.00009 & \multirow{2}{*}{3,240} \\
\hline & +0.25 wt. $\%$ & -0.00455 & \pm & 0.00016 & $\sqrt{3}$ & -0.00263 & \pm & 0.00009 & \\
\hline
\end{tabular}

Table 2.57. Effect of Uncertainty in BP Absorber Content (nominal 2.50 wt.\%).

\begin{tabular}{|c|c|c|c|c|c|c|c|c|c|}
\hline Case & Deviation & $\Delta \mathrm{k}$ & \pm & $\sigma_{\Delta \mathrm{k}}$ & $\begin{array}{l}\text { Scaling } \\
\text { Factor }\end{array}$ & $\Delta \mathrm{k}_{\mathrm{eff}}(1 \sigma)$ & \pm & $\sigma_{\Delta \mathrm{keff}}$ & $\mathrm{N}$ \\
\hline \multirow{2}{*}{1} & -0.25 wt. $\%$ & 0.00250 & \pm & 0.00017 & $\sqrt{3}$ & 0.00144 & \pm & 0.00010 & \multirow{2}{*}{1,216} \\
\hline & +0.25 wt. $\%$ & -0.00240 & - & 0.00017 & $\sqrt{3}$ & -0.00139 & \pm & 0.00010 & \\
\hline \multirow{2}{*}{2} & -0.25 wt. $\%$ & 0.00284 & \pm & 0.00016 & $\sqrt{3}$ & 0.00164 & \pm & 0.00009 & \multirow{2}{*}{1,344} \\
\hline & +0.25 wt. $\%$ & -0.00249 & & 0.00017 & $\sqrt{3}$ & -0.00144 & \pm & 0.00010 & \\
\hline \multirow{2}{*}{3} & -0.25 wt. $\%$ & 0.00249 & $\perp$ & 0.00017 & $\sqrt{3}$ & 0.00144 & \pm & 0.00010 & \multirow{2}{*}{1,536} \\
\hline & +0.25 wt. $\%$ & -0.00240 & - & 0.00016 & $\sqrt{3}$ & -0.00139 & \pm & 0.00009 & \\
\hline \multirow{2}{*}{4} & -0.25 wt. $\%$ & 0.00284 & \pm & 0.00017 & $\sqrt{3}$ & 0.00164 & \pm & 0.00010 & \multirow{2}{*}{1,536} \\
\hline & +0.25 wt. $\%$ & -0.00251 & 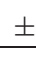 & 0.00016 & $\sqrt{3}$ & -0.00145 & \pm & 0.00009 & \\
\hline & -0.25 wt. $\%$ & 0.00200 & & 0.00016 & $\sqrt{3}$ & 0.00115 & \pm & 0.00009 & \multirow{2}{*}{1,728} \\
\hline & +0.25 wt. $\%$ & -0.00187 & \pm & 0.00016 & $\sqrt{3}$ & -0.00108 & \pm & 0.00009 & \\
\hline
\end{tabular}

\section{Absorber Impurity}

No information was available regarding any impurities present in the sintered $\mathrm{B}_{4} \mathrm{C} / \mathrm{C}$ material for the $\mathrm{BP}$ pellets. However, the impurity limits provided in Table 1.29 of HTTR-GCR-RESR-001 can be applied to approximate a rough estimate of the impact of additional impurities. Sodium and manganese were added with a concentrations of 100 and $10 \mathrm{ppm}$ (by weight), respectively. Concentrations of $1000 \mathrm{ppm}$ (by weight) of aluminum, silicon, calcium, and titanium were also included. The effective uncertainty in $\mathrm{k}_{\text {eff }}$ was determined and is shown in Table 2.58. This value is treated as a bounding limit.

The uncertainty in the absorber impurity is considered all systematic with no random component. 
NEA/NSC/DOC(2006)1

Gas Cooled (Thermal) Reactor - GCR

HTTR-GCR-RESR-002

CRIT-REAC-RRATE

Table 2.58. Effect of Uncertainty in Absorber Impurity.

\begin{tabular}{|c|c|ccc|c|ccc||}
\hline \hline Case & Deviation & $\Delta \mathrm{k}$ & \pm & $\sigma_{\Delta \mathrm{k}}$ & $\begin{array}{c}\text { Scaling } \\
\text { Factor }\end{array}$ & $\Delta \mathrm{k}_{\text {eff }}(1 \sigma)$ & \pm & $\sigma_{\Delta \mathrm{keff}}$ \\
\hline \hline 1 & Added Impurities & 0.00048 & \pm & 0.00016 & $2 \sqrt{3}$ & 0.00014 & \pm & 0.00005 \\
\hline 2 & Added Impurities & 0.00081 & \pm & 0.00017 & $2 \sqrt{3}$ & 0.00023 & \pm & 0.00005 \\
\hline 3 & Added Impurities & 0.00072 & \pm & 0.00016 & $2 \sqrt{3}$ & 0.00021 & \pm & 0.00005 \\
\hline 4 & Added Impurities & 0.00064 & \pm & 0.00017 & $2 \sqrt{ } 3$ & 0.00018 & \pm & 0.00005 \\
\hline 5 & Added Impurities & 0.00082 & \pm & 0.00016 & $2 \sqrt{3}$ & 0.00024 & \pm & 0.00004 \\
\hline \hline
\end{tabular}

\section{Absorber Isotopic Abundance}

According to the $16^{\text {th }}$ edition of the Chart of the Nuclides, the natural isotopic abundance of ${ }^{10} \mathrm{~B}$ has been measured between 19.1 and 20.3 at.\% with a nominal value of 19.9 at. $\%{ }^{a}$ The abundance of ${ }^{10} \mathrm{~B}$ in the BPs was therefore evaluated at the minimum and maximum bounding values to determine the effective uncertainty in $\mathrm{k}_{\text {eff. }}$ Table 1.28 of HTTR-GCR-RESR-001 states that the abundance of ${ }^{10} \mathrm{~B}$ is $18.7 \mathrm{wt} . \%$, which correlates to approximately 20.2 at.\%. The benchmark model was evaluated at 19.9 at.\%. Results are shown in Table 2.58 .

The uncertainty in the absorber isotopic abundance is considered all systematic with no random component.

Table 2.58. Effect of Uncertainty in BP Isotopic Abundance of ${ }^{10} \mathrm{~B}$.

\begin{tabular}{||c|c|ccc|c|ccc||}
\hline \hline Case & Deviation & $\Delta \mathrm{k}$ & \pm & $\sigma_{\Delta \mathrm{k}}$ & $\begin{array}{c}\text { Scaling } \\
\text { Factor }\end{array}$ & $\Delta_{\text {eff }}(1 \sigma)$ & \pm & $\sigma_{\Delta \mathrm{keff}}$ \\
\hline \hline \multirow{2}{*}{1} & $19.1 \%$ & 0.00178 & \pm & 0.00016 & $\sqrt{ } 3$ & 0.00014 & \pm & 0.00005 \\
& $20.3 \%$ & -0.00101 & \pm & 0.00016 & $\sqrt{ } 3$ & -0.00058 & \pm & 0.00009 \\
\hline \multirow{2}{*}{2} & $19.1 \%$ & 0.00227 & \pm & 0.00017 & $\sqrt{ } 3$ & 0.00131 & \pm & 0.00010 \\
& $20.3 \%$ & -0.00073 & \pm & 0.00017 & $\sqrt{ } 3$ & -0.00042 & \pm & 0.00010 \\
\hline \multirow{2}{*}{3} & $19.1 \%$ & 0.00219 & \pm & 0.00017 & $\sqrt{3}$ & 0.00126 & \pm & 0.00010 \\
& $20.3 \%$ & -0.00107 & \pm & 0.00016 & $\sqrt{3}$ & -0.00062 & \pm & 0.00009 \\
\hline \multirow{3}{*}{4} & $19.1 \%$ & 0.00220 & \pm & 0.00016 & $\sqrt{3}$ & 0.00127 & \pm & 0.00009 \\
& $20.3 \%$ & -0.00139 & \pm & 0.00017 & $\sqrt{3}$ & -0.00080 & \pm & 0.00010 \\
\hline \multirow{2}{*}{5} & $19.1 \%$ & 0.00236 & \pm & 0.00016 & $\sqrt{ } 3$ & 0.00136 & \pm & 0.00009 \\
& $20.3 \%$ & -0.00133 & \pm & 0.00016 & $\sqrt{3}$ & -0.00077 & \pm & 0.00009 \\
\hline
\end{tabular}

\section{Graphite Disk Density}

An uncertainty in the density of the graphite disks was not reported. A variation of $\pm 0.09 \mathrm{~g} / \mathrm{cm}^{3}$ was assumed and the effects on the uncertainty of $\mathrm{k}_{\mathrm{eff}}$ were determined. This value is three times the bounding limit. The graphite disks are composed of IG-110 graphite, and the assumed uncertainty of

${ }^{a}$ Nuclides and Isotopes: Chart of the Nuclides, $16^{\text {th }}$ edition, (2002).

Revision: 0 
NEA/NSC/DOC(2006)1

Gas Cooled (Thermal) Reactor - GCR

HTTR-GCR-RESR-002

CRIT-REAC-RRATE

$0.03 \mathrm{~g} / \mathrm{cm}^{3}$ encompasses the range of reported densities for IG-110 graphite throughout Section 1 of HTTR-GCR-RESR-001. Results are shown in Table 2.59.

The total number of graphite disk stacks used in the fully-loaded core is 300 . For determining the random component of the uncertainty, the results in Table 2.59 would be divided by $\sqrt{ } \mathrm{N}$, where $\mathrm{N}$ for each case is shown in Table 2.59.

Table 2.59. Effect of Uncertainty in Graphite Disk Density.

\begin{tabular}{|c|c|c|c|c|c|c|c|c|c|}
\hline Case & Deviation & $\Delta \mathrm{k}$ & \pm & $\sigma_{\Delta \mathrm{k}}$ & $\begin{array}{l}\text { Scaling } \\
\text { Factor }\end{array}$ & $\Delta \mathrm{k}_{\mathrm{eff}}(1 \sigma)$ & \pm & $\sigma_{\Delta \mathrm{keff}}$ & $\mathrm{N}$ \\
\hline \multirow{2}{*}{1} & $-0.09 \mathrm{~g} / \mathrm{cm}^{3}$ & 0.00015 & \pm & 0.00017 & $3 \sqrt{3}$ & 0.00003 & \pm & 0.00003 & \multirow{2}{*}{190} \\
\hline & $+0.09 \mathrm{~g} / \mathrm{cm}^{3}$ & 0.00003 & & 0.00016 & $3 \sqrt{3}$ & 0.00001 & \pm & 0.00003 & \\
\hline \multirow{2}{*}{2} & $-0.09 \mathrm{~g} / \mathrm{cm}^{3}$ & 0.00008 & \pm & 0.00017 & $3 \sqrt{3}$ & 0.000004 & \pm & 0.00003 & \multirow{2}{*}{210} \\
\hline & $+0.09 \mathrm{~g} / \mathrm{cm}^{3}$ & 0.00022 & & 0.00017 & $3 \sqrt{3}$ & 0.00004 & \pm & 0.00003 & \\
\hline \multirow{2}{*}{3} & $-0.09 \mathrm{~g} / \mathrm{cm}^{3}$ & -0.00017 & \pm & 0.00017 & $3 \sqrt{3}$ & -0.00003 & \pm & 0.00003 & \multirow{2}{*}{240} \\
\hline & $+0.09 \mathrm{~g} / \mathrm{cm}^{3}$ & -0.00005 & & 0.00017 & $3 \sqrt{3}$ & -0.00001 & \pm & 0.00003 & \\
\hline \multirow{2}{*}{4} & $-0.09 \mathrm{~g} / \mathrm{cm}^{3}$ & -0.00027 & \pm & 0.00017 & $3 \sqrt{3}$ & -0.00005 & \pm & 0.00003 & \multirow{2}{*}{240} \\
\hline & $+0.09 \mathrm{~g} / \mathrm{cm}^{3}$ & -0.00006 & & 0.00017 & $3 \sqrt{3}$ & -0.00001 & \pm & 0.00003 & \\
\hline \multirow{2}{*}{5} & $-0.09 \mathrm{~g} / \mathrm{cm}^{3}$ & 0.00001 & & 0.00016 & $3 \sqrt{3}$ & 0.00000 & \pm & 0.00003 & \multirow{2}{*}{270} \\
\hline & $+0.09 \mathrm{~g} / \mathrm{cm}^{3}$ & 0.00001 & \pm & 0.00016 & $3 \sqrt{3}$ & 0.00000 & \pm & 0.00003 & \\
\hline
\end{tabular}

\section{Graphite Disk Impurity}

The assumed graphite disk impurity was varied from 0-5 ppm by weight of equivalent natural-boron content to determine the bounding uncertainty in $\mathrm{k}_{\text {eff. }}$ The nominal impurity is $0.37 \mathrm{ppm}$ of natural boron by weight (Table 1.28 of HTTR-GCR-RESR-001). Results are shown in Table 2.60.

The uncertainty in the graphite disk impurity is considered all systematic with no random component. 
NEA/NSC/DOC(2006)1

Gas Cooled (Thermal) Reactor - GCR

HTTR-GCR-RESR-002

CRIT-REAC-RRATE

Table 2.60. Effect of Uncertainty in Graphite Disk Impurity.

\begin{tabular}{||c|c|ccc|c|ccc||}
\hline \multirow{2}{*}{ Case } & Deviation & $\Delta \mathrm{k}$ & \pm & $\sigma_{\Delta \mathrm{k}}$ & $\begin{array}{c}\text { Scaling } \\
\text { Factor }\end{array}$ & $\Delta \mathrm{k}_{\text {eff }}(1 \sigma)$ & \pm & $\sigma_{\Delta \mathrm{keff}}$ \\
\hline \hline \multirow{2}{*}{1} & $0 \mathrm{ppm}$ & -0.00018 & \pm & 0.00017 & $\sqrt{ } 3$ & -0.00010 & \pm & 0.00010 \\
& $5 \mathrm{ppm}$ & -0.00006 & \pm & 0.00017 & $\sqrt{ } 3$ & -0.00003 & \pm & 0.00010 \\
\hline \multirow{2}{*}{2} & $0 \mathrm{ppm}$ & 0.00004 & \pm & 0.00016 & $\sqrt{ } 3$ & 0.00002 & \pm & 0.00009 \\
& $5 \mathrm{ppm}$ & 0.00016 & \pm & 0.00017 & $\sqrt{ } 3$ & 0.00009 & \pm & 0.00010 \\
\hline \multirow{3}{*}{3} & $0 \mathrm{ppm}$ & -0.00016 & \pm & 0.00017 & $\sqrt{3}$ & -0.00009 & \pm & 0.00010 \\
& $5 \mathrm{ppm}$ & -0.00017 & \pm & 0.00016 & $\sqrt{3}$ & -0.00010 & \pm & 0.00009 \\
\hline \multirow{3}{*}{4} & $0 \mathrm{ppm}$ & -0.00017 & \pm & 0.00017 & $\sqrt{3}$ & -0.00010 & \pm & 0.00010 \\
& $5 \mathrm{ppm}$ & -0.00007 & \pm & 0.00017 & $\sqrt{3}$ & -0.00004 & \pm & 0.00010 \\
\hline \multirow{2}{*}{5} & $0 \mathrm{ppm}$ & -0.00001 & \pm & 0.00016 & $\sqrt{3}$ & -0.00001 & \pm & 0.00009 \\
& $5 \mathrm{ppm}$ & -0.00011 & \pm & 0.00016 & $\sqrt{3}$ & -0.00006 & \pm & 0.00009 \\
\hline
\end{tabular}

\subsubsection{Control Rods}

\section{Absorber Density}

The absorber density was varied $\pm 0.09 \mathrm{~g} / \mathrm{cm}^{3}$ from the nominal value of $1.9 \mathrm{~g} / \mathrm{cm}^{3}$ (Table 1.15 of HTTR-GCR-RESR-001) to determine the effective uncertainty in $\mathrm{k}_{\mathrm{eff}}$. This value is three times the bounding limit. The uncertainty is taken from Table 1.29 of HTTR-GCR-RESR-001. Results are shown in Table 2.46 .

The total number of control rod absorber pellets used in the fully-loaded core is approximately 975 , as approximately $30 \%$ of the control rods are actually inserted into the core. For determining the random component of the uncertainty, the results in Table 2.61 would be divided by $\sqrt{ } \mathrm{N}$, where $\mathrm{N}$ for each case is shown in Table 2.61.

Table 2.61. Effect of Uncertainty in CR Absorber Density.

\begin{tabular}{|c|c|c|c|c|c|c|c|c|c|}
\hline Case & Deviation & $\Delta \mathrm{k}$ & \pm & $\sigma_{\Delta \mathrm{k}}$ & $\begin{array}{l}\text { Scaling } \\
\text { Factor }\end{array}$ & $\Delta \mathrm{k}_{\mathrm{eff}}(1 \sigma)$ & \pm & $\sigma_{\Delta \mathrm{keff}}$ & $\mathrm{N}$ \\
\hline \multirow{2}{*}{1} & $-0.09 \mathrm{~g} / \mathrm{cm}^{3}$ & 0.00004 & \pm & 0.00017 & $3 \sqrt{3}$ & 0.00001 & \pm & 0.00003 & \multirow{2}{*}{225} \\
\hline & $+0.09 \mathrm{~g} / \mathrm{cm}^{3}$ & -0.00023 & \pm & 0.00016 & $3 \sqrt{3}$ & 0.00003 & \pm & 0.00003 & \\
\hline \multirow{2}{*}{2} & $-0.09 \mathrm{~g} / \mathrm{cm}^{3}$ & 0.00013 & \pm & 0.00016 & $3 \sqrt{ } 3$ & 0.00003 & \pm & 0.00003 & \multirow{2}{*}{585} \\
\hline & $+0.09 \mathrm{~g} / \mathrm{cm}^{3}$ & 0.00007 & \pm & 0.00016 & $3 \sqrt{3}$ & 0.00001 & \pm & 0.00003 & \\
\hline \multirow{2}{*}{3} & $-0.09 \mathrm{~g} / \mathrm{cm}^{3}$ & 0.00020 & \pm & 0.00016 & $3 \sqrt{3}$ & 0.00004 & \pm & 0.00003 & \multirow{2}{*}{780} \\
\hline & $+0.09 \mathrm{~g} / \mathrm{cm}^{3}$ & -0.00009 & \pm & 0.00016 & $3 \sqrt{3}$ & -0.00002 & \pm & 0.00003 & \\
\hline \multirow{2}{*}{4} & $-0.09 \mathrm{~g} / \mathrm{cm}^{3}$ & -0.00002 & \pm & 0.00016 & $3 \sqrt{3}$ & 0.00000 & \pm & 0.00003 & \multirow{2}{*}{720} \\
\hline & $+0.09 \mathrm{~g} / \mathrm{cm}^{3}$ & -0.00025 & \pm & 0.00016 & $3 \sqrt{3}$ & -0.00005 & \pm & 0.00003 & \\
\hline \multirow{2}{*}{5} & $-0.09 \mathrm{~g} / \mathrm{cm}^{3}$ & -0.00013 & \pm & 0.00016 & $3 \sqrt{ } 3$ & -0.00003 & \pm & 0.00003 & \multirow{2}{*}{910} \\
\hline & $+0.09 \mathrm{~g} / \mathrm{cm}^{3}$ & -0.00024 & \pm & 0.00016 & $3 \sqrt{3}$ & -0.00005 & \pm & 0.00003 & \\
\hline
\end{tabular}

Revision: 0 
NEA/NSC/DOC(2006)1

Gas Cooled (Thermal) Reactor - GCR

HTTR-GCR-RESR-002

CRIT-REAC-RRATE

\section{Absorber Content}

The boron content was varied \pm 9 wt.\% from the nominal value of 30 wt.\% (Table 1.15 of HTTR-GCR-RESR-001) and the effect on the uncertainty in $\mathrm{k}_{\text {eff }}$ was determined. This value is three times the bounding limit. The uncertainty is taken from Table 1.29 of HTTR-GCR-RESR-001. Results are shown in Table 2.62.

The total number of control rod absorber pellets used in the fully-loaded core is approximately 975, as approximately $30 \%$ of the control rods are actually inserted into the core. For determining the random component of the uncertainty, the results in Table 2.62 would be divided by $\sqrt{ } \mathrm{N}$, where $\mathrm{N}$ for each case is shown in Table 2.62.

Table 2.62. Effect of Uncertainty in CR Absorber Content.

\begin{tabular}{|c|c|c|c|c|c|c|c|c|c|}
\hline Case & Deviation & $\Delta \mathrm{k}$ & \pm & $\sigma_{\Delta \mathrm{k}}$ & $\begin{array}{l}\text { Scaling } \\
\text { Factor } \\
\end{array}$ & $\Delta \mathrm{k}_{\mathrm{eff}}(1 \sigma)$ & \pm & $\sigma_{\Delta \mathrm{keff}}$ & $\mathrm{N}$ \\
\hline \multirow{2}{*}{1} & -9 wt. $\%$ & 0.00002 & \pm & 0.00017 & $3 \sqrt{3}$ & 0.00000 & \pm & 0.00003 & \multirow{2}{*}{225} \\
\hline & +9 wt. $\%$ & -0.00016 & \pm & 0.00017 & $3 \sqrt{3}$ & -0.00003 & \pm & 0.00003 & \\
\hline \multirow{2}{*}{2} & -9 wt. $\%$ & 0.00055 & \pm & 0.00017 & $3 \sqrt{3}$ & 0.00011 & \pm & 0.00003 & \multirow{2}{*}{585} \\
\hline & +9 wt. $\%$ & -0.00030 & \pm & 0.00016 & $3 \sqrt{3}$ & -0.00006 & \pm & 0.00003 & \\
\hline \multirow{2}{*}{3} & -9 wt. $\%$ & 0.00045 & \pm & 0.00017 & $3 \sqrt{3}$ & 0.00009 & \pm & 0.00003 & \multirow{2}{*}{780} \\
\hline & +9 wt. $\%$ & -0.00045 & \pm & 0.00017 & $3 \sqrt{3}$ & -0.00009 & \pm & 0.00003 & \\
\hline \multirow{2}{*}{4} & -9 wt. $\%$ & 0.00064 & \pm & 0.00017 & $3 \sqrt{3}$ & 0.00012 & \pm & 0.00003 & \multirow{2}{*}{720} \\
\hline & +9 & -0.00066 & \pm & 0.00017 & $3 \sqrt{3}$ & -0.00013 & \pm & 0.00003 & \\
\hline & -9 wt.\% & 0.00045 & \pm & 0.00016 & $3 \sqrt{3}$ & 0.00009 & \pm & 0.00003 & \multirow{2}{*}{910} \\
\hline & +9 wt. $\%$ & -0.00053 & \pm & 0.00016 & $3 \sqrt{3}$ & -0.00010 & \pm & 0.00003 & \\
\hline
\end{tabular}

\section{Absorber Impurity}

No information was available regarding any impurities present in the sintered $\mathrm{B}_{4} \mathrm{C} / \mathrm{C}$ material for the control rod absorbers. However, the impurity limits provided in Table 1.29 of HTTR-GCR-RESR-001 can be applied to approximate a rough estimate of the impact of additional impurities. Sodium and manganese were added with a concentrations of 100 and $10 \mathrm{ppm}$ (by weight), respectively. Concentrations of $1000 \mathrm{ppm}$ (by weight) of aluminum, silicon, calcium, and titanium were also included. The effective uncertainty in $\mathrm{k}_{\text {eff }}$ was determined and is shown in Table 2.63. This value is treated as a bounding limit.

The uncertainty in the absorber impurity is considered all systematic with no random component. 
NEA/NSC/DOC(2006)1

Gas Cooled (Thermal) Reactor - GCR

HTTR-GCR-RESR-002

CRIT-REAC-RRATE

Table 2.63. Effect of Uncertainty in Absorber Impurity.

\begin{tabular}{||c|c|ccc|c|ccc||}
\hline \hline Case & Deviation & $\Delta \mathrm{k}$ & \pm & $\sigma_{\Delta \mathrm{k}}$ & $\begin{array}{c}\text { Scaling } \\
\text { Factor }\end{array}$ & $\Delta \mathrm{k}_{\text {eff }}(1 \sigma)$ & \pm & $\sigma_{\Delta \mathrm{keff}}$ \\
\hline \hline 1 & Added Impurities & -0.00007 & \pm & 0.00016 & $2 \sqrt{3}$ & -0.00002 & \pm & 0.00005 \\
\hline 2 & Added Impurities & -0.00017 & \pm & 0.00016 & $2 \sqrt{3}$ & -0.00005 & \pm & 0.00005 \\
\hline 3 & Added Impurities & -0.00009 & \pm & 0.00017 & $2 \sqrt{ } 3$ & -0.00003 & \pm & 0.00005 \\
\hline 4 & Added Impurities & -0.00005 & \pm & 0.00017 & $2 \sqrt{3}$ & -0.00013 & \pm & 0.00003 \\
\hline 5 & Added Impurities & -0.00005 & \pm & 0.00016 & $2 \sqrt{ } 3$ & -0.00001 & \pm & 0.00004 \\
\hline \hline
\end{tabular}

\section{Absorber Isotopic Abundance}

According to the $16^{\text {th }}$ edition of the Chart of the Nuclides, the natural isotopic abundance of ${ }^{10} \mathrm{~B}$ has been measured between 19.1 and $20.3 \%$ with a nominal value of $19.9 \%$. ${ }^{a}$ The abundance of ${ }^{10} \mathrm{~B}$ in the control rods was therefore evaluated at the minimum and maximum bounding values to determine the effective uncertainty in $\mathrm{k}_{\text {eff. }}$ Table 1.28 of HTTR-GCR-RESR-001 states that the abundance of ${ }^{10} \mathrm{~B}$ is $18.7 \mathrm{wt} . \%$, which correlates to approximately 20.2 at.\%. The model was not evaluated for a ${ }^{10} \mathrm{~B}$ abundance of 18.7 wt.\%. The benchmark model was evaluated at 19.9 at.\%. Results are shown in Table 2.64.

The uncertainty in the absorber isotopic abundance is considered all systematic with no random component.

Table 2.64. Effect of Uncertainty in CR Isotopic Abundance of ${ }^{10} \mathrm{~B}$.

\begin{tabular}{||c|c|ccc|c|ccc||}
\hline \hline Case & Deviation & $\Delta \mathrm{k}$ & \pm & $\sigma_{\Delta \mathrm{k}}$ & $\begin{array}{c}\text { Scaling } \\
\text { Factor }\end{array}$ & $\Delta_{\text {eff }}(1 \sigma)$ & \pm & $\sigma_{\Delta \mathrm{keff}}$ \\
\hline \hline \multirow{2}{*}{1} & $19.1 \%$ & 0.00008 & \pm & 0.00017 & $\sqrt{ } 3$ & 0.00005 & \pm & 0.00010 \\
& $20.3 \%$ & 0.00013 & \pm & 0.00017 & $\sqrt{ } 3$ & 0.00008 & \pm & 0.00010 \\
\hline \multirow{2}{*}{2} & $19.1 \%$ & 0.00009 & \pm & 0.00017 & $\sqrt{ } 3$ & 0.00005 & \pm & 0.00010 \\
& $20.3 \%$ & 0.00000 & \pm & 0.00017 & $\sqrt{ } 3$ & 0.00000 & \pm & 0.00010 \\
\hline \multirow{2}{*}{3} & $19.1 \%$ & 0.00022 & \pm & 0.00016 & $\sqrt{3}$ & 0.00013 & \pm & 0.00009 \\
& $20.3 \%$ & -0.00009 & \pm & 0.00016 & $\sqrt{3}$ & -0.00005 & \pm & 0.00009 \\
\hline \multirow{3}{*}{4} & $19.1 \%$ & -0.00013 & \pm & 0.00017 & $\sqrt{3}$ & -0.00008 & \pm & 0.00010 \\
& $20.3 \%$ & -0.00018 & \pm & 0.00016 & $\sqrt{3}$ & -0.00010 & \pm & 0.00009 \\
\hline \multirow{2}{*}{5} & $19.1 \%$ & -0.00011 & \pm & 0.00016 & $\sqrt{ } 3$ & -0.00006 & \pm & 0.00009 \\
& $20.3 \%$ & 0.00036 & \pm & 0.00016 & $\sqrt{3}$ & 0.00021 & \pm & 0.00009 \\
\hline
\end{tabular}

\footnotetext{
${ }^{\text {a }}$ Nuclides and Isotopes: Chart of the Nuclides, $16^{\text {th }}$ edition, (2002).
} 
NEA/NSC/DOC(2006)1

\section{Gas Cooled (Thermal) Reactor - GCR \\ HTTR-GCR-RESR-002 \\ CRIT-REAC-RRATE}

\section{Clad Density}

Two references were compared to confirm the composition and density of the Alloy $800 \mathrm{H}$ material. ${ }^{\text {ab }}$ The density of Alloy $800 \mathrm{H}$ is taken to be $8.03 \mathrm{~g} / \mathrm{cm}^{3}$ with an estimated uncertainty of $\pm 0.03 \mathrm{~g} / \mathrm{cm}^{3}$, which is the difference between the reported density values in the two references. This value is the bounding limit. The effective change in $\mathrm{k}_{\text {eff }}$ was determined. Results are shown in Table 2.65.

The total number of control rod sections used in the fully-loaded core is approximately 97.5, as approximately $30 \%$ of the control rods are actually inserted into the core. For determining the random component of the uncertainty, the results in Table 2.65 would be divided by $\sqrt{ } \mathrm{N}$, where $\mathrm{N}$ for each case is shown in Table 2.65.

Table 2.65. Effect of Uncertainty in Clad Density.

\begin{tabular}{|c|c|c|c|c|c|c|c|c|c|}
\hline Case & Deviation & $\Delta \mathrm{k}$ & \pm & $\sigma_{\Delta \mathrm{k}}$ & $\begin{array}{l}\text { Scaling } \\
\text { Factor }\end{array}$ & $\Delta \mathrm{k}_{\mathrm{eff}}(1 \sigma)$ & \pm & $\sigma_{\Delta \mathrm{keff}}$ & $\mathrm{N}$ \\
\hline \multirow{2}{*}{1} & $-0.03 \mathrm{~g} / \mathrm{cm}^{3}(1 \sigma)$ & 0.00028 & \pm & 0.00017 & $\sqrt{3}$ & 0.00016 & \pm & 0.00010 & \multirow{2}{*}{22.5} \\
\hline & $+0.03 \mathrm{~g} / \mathrm{cm}^{3}(1 \sigma)$ & -0.00012 & \pm & 0.00016 & $\sqrt{3}$ & -0.00007 & \pm & 0.00009 & \\
\hline \multirow{2}{*}{2} & $-0.03 \mathrm{~g} / \mathrm{cm}^{3}(1 \sigma)$ & 0.00012 & \pm & 0.00017 & $\sqrt{3}$ & 0.00007 & \pm & 0.00010 & \multirow{2}{*}{58.5} \\
\hline & $+0.03 \mathrm{~g} / \mathrm{cm}^{3}(1 \sigma)$ & 0.00001 & \pm & 0.00017 & $\sqrt{3}$ & 0.00001 & \pm & 0.00010 & \\
\hline \multirow[b]{2}{*}{3} & $-0.03 \mathrm{~g} / \mathrm{cm}^{3}(1 \sigma)$ & 0.00006 & \pm & 0.00016 & $\sqrt{3}$ & 0.00003 & \pm & 0.00009 & \multirow{2}{*}{78.0} \\
\hline & $+0.03 \mathrm{~g} / \mathrm{cm}^{3}(1 \sigma)$ & -0.00023 & 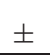 & 0.00016 & $\sqrt{3}$ & -0.00013 & \pm & 0.00009 & \\
\hline \multirow{2}{*}{4} & $-0.03 \mathrm{~g} / \mathrm{cm}^{3}(1 \sigma)$ & -0.00032 & \pm & 0.00017 & $\sqrt{3}$ & -0.00018 & \pm & 0.00010 & \multirow{2}{*}{72.0} \\
\hline & $+0.03 \mathrm{~g} / \mathrm{cm}^{3}(1 \sigma)$ & -0.00005 & \pm & 0.00017 & $\sqrt{3}$ & -0.00003 & \pm & 0.00010 & \\
\hline & $-0.03 \mathrm{~g} / \mathrm{cm}^{3}(1 \sigma)$ & 0.00004 & \pm & 0.00016 & $\sqrt{3}$ & 0.00002 & \pm & 0.00009 & \multirow{2}{*}{91.0} \\
\hline & $+0.03 \mathrm{~g} / \mathrm{cm}^{3}(1 \sigma)$ & 0.00007 & \pm & 0.00016 & $\sqrt{3}$ & 0.00004 & \pm & 0.00009 & \\
\hline
\end{tabular}

\section{Clad Composition}

The clad composition used in the benchmark model is derived from the aforementioned references for Alloy $800 \mathrm{H}$, where the second reference provides a composition range and the first reference provides a nominal composition. A summary of the composition of Alloy $800 \mathrm{H}$ is provided in Table 2.66. The nominal distribution is used in the benchmark model. The composition of the clad material was varied from the nominal value. In the first case, the minimum compositions of all elements were used with iron as the remaining balance. The second case uses the maximum weights of all the elements with a reduction in the iron content until the minimum value is achieved; then nickel is reduced to achieve a total of $100 \mathrm{wt} . \%$. The effective change in $\mathrm{k}_{\mathrm{eff}}$ for these two cases was determined. Results are shown in Table 2.67 .

The uncertainty in the clad composition is considered all systematic with no random component.

\footnotetext{
${ }^{a}$ Specification Sheet: Alloy 800, 800H, and 800AT, Sandmeyer Steel Company (April 2004).

${ }^{\mathrm{b}}$ Material Characteristics: Alloy 800H, PhilipCornes, http://www.cornes.com.sg/a800h.htm (Accessed August 5, 2008).
} 
NEA/NSC/DOC(2006)1

Gas Cooled (Thermal) Reactor - GCR

HTTR-GCR-RESR-002

CRIT-REAC-RRATE

Table 2.66. Composition of Alloy 800H.

\begin{tabular}{||c|c|c|c||}
\hline \hline Element & Minimum wt.\% & Maximum wt.\% & Nominal wt.\% \\
$\mathrm{C}$ & 0.06 & 0.1 & 0.08 \\
$\mathrm{Al}$ & 0.15 & 0.6 & 0.375 \\
$\mathrm{Si}$ & -- & 1.0 & 0.35 \\
$\mathrm{P}$ & -- & -- & 0.02 \\
$\mathrm{~S}$ & -- & 0.015 & 0.01 \\
$\mathrm{Ti}$ & 0.15 & 0.6 & 0.375 \\
$\mathrm{Cr}$ & 19 & 23 & 21 \\
$\mathrm{Mn}$ & -- & 1.5 & 1 \\
$\mathrm{Fe}$ & 39.5 & -- & 43.99 \\
$\mathrm{Ni}$ & 30 & 35 & 32.5 \\
$\mathrm{Cu}$ & -- & 0.75 & 0.3 \\
\hline \hline $\mathrm{Total}$ & -- & -- & 100.000 \\
\hline \hline
\end{tabular}

Table 2.67. Effect of Uncertainty in Clad Composition.

\begin{tabular}{||c|c|ccc|c|ccc||}
\hline \hline \multirow{2}{*}{ Case } & Deviation & $\Delta \mathrm{k}$ & \pm & $\sigma_{\Delta \mathrm{k}}$ & $\begin{array}{c}\text { Scaling } \\
\text { Factor }\end{array}$ & $\Delta \mathrm{k}_{\text {eff }}(1 \sigma)$ & \pm & $\sigma_{\Delta \mathrm{keff}}$ \\
\hline \hline \multirow{2}{*}{1} & Minimum Fe & -0.00010 & \pm & 0.00016 & $\sqrt{ } 3$ & -0.00006 & \pm & 0.00009 \\
& Maximum Fe & -0.00004 & \pm & 0.00016 & $\sqrt{ } 3$ & -0.00002 & \pm & 0.00009 \\
\hline \multirow{2}{*}{2} & Minimum Fe & 0.00014 & \pm & 0.00017 & $\sqrt{ } 3$ & 0.00009 & \pm & 0.00010 \\
& Maximum Fe & 0.00016 & \pm & 0.00017 & $\sqrt{ } 3$ & 0.00009 & \pm & 0.00010 \\
\hline \multirow{2}{*}{3} & Minimum Fe & -0.00009 & \pm & 0.00017 & $\sqrt{ } 3$ & -0.00005 & \pm & 0.00010 \\
& Maximum Fe & -0.00020 & \pm & 0.00016 & $\sqrt{ } 3$ & -0.00012 & \pm & 0.00009 \\
\hline \multirow{2}{*}{4} & Minimum Fe & -0.00014 & \pm & 0.00017 & $\sqrt{3}$ & -0.00008 & \pm & 0.00010 \\
& Maximum Fe & -0.00012 & \pm & 0.00017 & $\sqrt{ } 3$ & -0.00007 & \pm & 0.00010 \\
\hline \multirow{2}{*}{5} & Minimum Fe & -0.00012 & \pm & 0.00016 & $\sqrt{3}$ & -0.00007 & \pm & 0.00009 \\
& Maximum Fe & -0.00007 & \pm & 0.00016 & $\sqrt{ } 3$ & -0.00004 & \pm & 0.00009 \\
\hline \hline
\end{tabular}

\section{Clad Impurity}

No information was available regarding any impurities present in the Alloy $800 \mathrm{H}$ metal. Concentrations of minor and trace elements found commonly in nickel alloy ${ }^{\mathrm{a}}$ were added to the clad material in the benchmark model (a neutron cross-section library was unavailable for Ytterbium) to determine the effective uncertainty in $k_{\text {eff. }}$. Because these impurities are not necessarily those that would have been in the alloy, and the total amount is $\sim 10 \mathrm{wt} . \%$, the atom density of the primary alloy composition was not

a J. H. Zaidi, S. Waheed, and S. Ahmed, "Determination of Trace Impurities in Nickel-Based Alloy using Neutron Activation Analysis,” J. Radioanal. Nucl. Ch., 242: 259-263 (1999). 
NEA/NSC/DOC(2006)1

Gas Cooled (Thermal) Reactor - GCR

HTTR-GCR-RESR-002

CRIT-REAC-RRATE

reduced. Thus the total atom density of the material was increased by including the additional materials. A list of the additional impurities is shown in Table 2.68, and results are shown in Table 2.69. This value is treated as a bounding limit.

The uncertainty in the clad impurity is considered all systematic with no random component.

Table 2.68. Concentration (by weight) of Minor and Trace Elements in Nickel Alloy.

\begin{tabular}{|c|c||c|c||}
\hline \hline Element & Concentration & Element & Concentration \\
\cline { 1 - 1 } $\mathrm{As}$ & $63.7 \mu \mathrm{g} / \mathrm{g}$ & $\mathrm{Mo}$ & $9.8 \mu \mathrm{g} / \mathrm{g}$ \\
$\mathrm{Ba}$ & $215 \mu \mathrm{g} / \mathrm{g}$ & $\mathrm{Na}$ & $0.45 \%$ \\
$\mathrm{Br}$ & $8.9 \mu \mathrm{g} / \mathrm{g}$ & $\mathrm{Nd}$ & $16.2 \mu \mathrm{g} / \mathrm{g}$ \\
$\mathrm{Ca}$ & $5.9 \%$ & $\mathrm{Rb}$ & $24 \mathrm{ng} / \mathrm{g}$ \\
$\mathrm{Ce}$ & $0.33 \%$ & $\mathrm{Sb}$ & $2.2 \mu \mathrm{g} / \mathrm{g}$ \\
$\mathrm{Co}$ & $9.96 \mu \mathrm{g} / \mathrm{g}$ & $\mathrm{Sc}$ & $0.13 \mu \mathrm{g} / \mathrm{g}$ \\
$\mathrm{Cs}$ & $4.34 \mu \mathrm{g} / \mathrm{g}$ & $\mathrm{Se}$ & $0.16 \mu \mathrm{g} / \mathrm{g}$ \\
$\mathrm{Dy}$ & $6.12 \mu \mathrm{g} / \mathrm{g}$ & $\mathrm{Sm}$ & $0.23 \mu \mathrm{g} / \mathrm{g}$ \\
$\mathrm{Er}$ & $2.8 \mu \mathrm{g} / \mathrm{g}$ & $\mathrm{Sn}$ & $0.25 \mu \mathrm{g} / \mathrm{g}$ \\
$\mathrm{Eu}$ & $1.17 \mu \mathrm{g} / \mathrm{g}$ & $\mathrm{Sr}$ & $371 \mu \mathrm{g} / \mathrm{g}$ \\
$\mathrm{Ga}$ & $107.5 \mu \mathrm{g} / \mathrm{g}$ & $\mathrm{Ta}$ & $1.21 \mu \mathrm{g} / \mathrm{g}$ \\
$\mathrm{Gd}$ & $1.7 \mu \mathrm{g} / \mathrm{g}$ & $\mathrm{Tb}$ & $1.24 \mu \mathrm{g} / \mathrm{g}$ \\
$\mathrm{Hf}$ & $19.5 \mu \mathrm{g} / \mathrm{g}$ & $\mathrm{Th}$ & $18.3 \mu \mathrm{g} / \mathrm{g}$ \\
$\mathrm{Hg}$ & $6 \mathrm{ng} / \mathrm{g}$ & $\mathrm{U}$ & $4.3 \mu \mathrm{g} / \mathrm{g}$ \\
$\mathrm{In}$ & $245 \mathrm{ng} / \mathrm{g}$ & $\mathrm{Yb}$ & $2.48 \mu \mathrm{g} / \mathrm{g}$ \\
$\mathrm{La}$ & $2.34 \mu \mathrm{g} / \mathrm{g}$ & $\mathrm{V}$ & $0.75 \%$ \\
$\mathrm{Lu}$ & $1.16 \mu \mathrm{g} / \mathrm{g}$ & $\mathrm{Zn}$ & $93.4 \mu \mathrm{g} / \mathrm{g}$ \\
$\mathrm{Mg}$ & $2.14 \%$ & & \\
\hline \hline
\end{tabular}

(a) A neutron cross-section library was unavailable to include this element in the analysis.

Table 2.69. Effect of Uncertainty in Clad Impurity.

\begin{tabular}{||c|c|ccc|c|ccc||}
\hline \hline Case & Deviation & $\Delta \mathrm{k}$ & \pm & $\sigma_{\Delta \mathrm{k}}$ & $\begin{array}{c}\text { Scaling } \\
\text { Factor }\end{array}$ & $\Delta \mathrm{k}_{\text {eff }}(1 \sigma)$ & \pm & $\sigma_{\Delta \mathrm{keff}}$ \\
\hline \hline 1 & Added Impurities & 0.00015 & \pm & 0.00016 & $2 \sqrt{ } 3$ & 0.00004 & \pm & 0.00005 \\
\hline 2 & Added Impurities & -0.00005 & \pm & 0.00016 & $2 \sqrt{ } 3$ & -0.00001 & \pm & 0.00005 \\
\hline 3 & Added Impurities & 0.00015 & \pm & 0.00016 & $2 \sqrt{ } 3$ & 0.00004 & \pm & 0.00005 \\
\hline 4 & Added Impurities & -0.00001 & \pm & 0.00016 & $2 \sqrt{ } 3$ & 0.00000 & \pm & 0.00005 \\
\hline 5 & Added Impurities & -0.00002 & \pm & 0.00016 & $2 \sqrt{ } 3$ & -0.00001 & \pm & 0.00004 \\
\hline
\end{tabular}


NEA/NSC/DOC(2006)1

\section{Gas Cooled (Thermal) Reactor - GCR \\ HTTR-GCR-RESR-002 \\ CRIT-REAC-RRATE}

\subsubsection{Instrumentation}

Insufficient information is available to model and comprehensively evaluate the uncertainties and biases related to the utility of instrumentation in the HTTR. A basic analysis of the general description of the instrumentation was performed, as discussed in Section 2.1.2.6.

\subsubsection{Graphite Blocks}

\section{Density}

The IG-110 graphite density was varied $\pm 0.04 \mathrm{~g} / \mathrm{cm}^{3}$ from a nominal value selected as $1.76 \mathrm{~g} / \mathrm{cm}^{3}$ (ranging from 1.75 to $1.78 \mathrm{~g} / \mathrm{cm}^{3}$, Tables $1.13,1.19$, and 1.27 of HTTR-GCR-RESR-001) to determine the effective uncertainty in $\mathrm{k}_{\text {eff. }}$. A variation of $0.03 \mathrm{~g} / \mathrm{cm}^{3}$ accounts for the difference in density between the various samples of IG-110 blocks, and a variation of $0.01 \mathrm{~g} / \mathrm{cm}^{3}$ is assumed to encompass the variability in the volume fraction caused by eliminating various features such as the dowels, sockets, fuel handling position, and ridged features on the fuel rods. The assumed uncertainty of $0.03 \mathrm{~g} / \mathrm{cm}^{3}$ for the IG-110 graphite encompasses the range of reported densities found throughout Section 1 of HTTR-GCR-RESR-001. Results are shown in Table 2.70. This value is treated as a bounding limit.

Previous results ${ }^{\mathrm{a}}$ state that a graphite weight difference of less than $1 \%$ should result in a bias of $-0.3 \%$ $\Delta \mathrm{k} / \mathrm{k}$. Scaling the results provided in Table 2.70 provides an uncertainty comparable to the previously published information.

The total number of IG-110 graphite blocks used in the fully-loaded core is 549. For determining the random component of the uncertainty, the results in Table 2.70 would be divided by $\sqrt{ } \mathrm{N}$, where $\mathrm{N}$ for each case is shown in Table 2.70.

Table 2.70. Effect of Uncertainty in IG-110 Density in Graphite Blocks.

\begin{tabular}{|c|c|c|c|c|c|c|c|c|c|}
\hline Case & Deviation & $\Delta \mathrm{k}$ & \pm & $\sigma_{\Delta \mathrm{k}}$ & $\begin{array}{l}\text { Scaling } \\
\text { Factor }\end{array}$ & $\Delta \mathrm{k}_{\mathrm{eff}}(1 \sigma)$ & \pm & $\sigma_{\Delta \mathrm{keff}}$ & $\mathrm{N}$ \\
\hline \multirow{2}{*}{1} & $-0.04 \mathrm{~g} / \mathrm{cm}^{3}$ & -0.00418 & \pm & 0.00016 & $\sqrt{3}$ & -0.00241 & \pm & 0.00009 & \multirow{2}{*}{494} \\
\hline & $+0.04 \mathrm{~g} / \mathrm{cm}^{3}$ & 0.00422 & \pm & 0.00017 & $\sqrt{3}$ & 0.00244 & \pm & 0.00010 & \\
\hline \multirow{2}{*}{2} & $-0.04 \mathrm{~g} / \mathrm{cm}^{3}$ & -0.00424 & \pm & 0.00016 & $\sqrt{3}$ & -0.00245 & \pm & 0.00009 & \multirow{2}{*}{504} \\
\hline & $+0.04 \mathrm{~g} / \mathrm{cm}^{3}$ & 0.00455 & \pm & 0.00016 & $\sqrt{3}$ & 0.00263 & \pm & 0.00009 & \\
\hline \multirow{2}{*}{3} & $-0.04 \mathrm{~g} / \mathrm{cm}^{3}$ & -0.00507 & \pm & 0.00016 & $\sqrt{3}$ & -0.00293 & \pm & 0.00009 & \multirow{2}{*}{519} \\
\hline & $+0.04 \mathrm{~g} / \mathrm{cm}^{3}$ & 0.00439 & \pm & 0.00016 & $\sqrt{3}$ & 0.00253 & \pm & 0.00009 & \\
\hline \multirow{2}{*}{4} & $-0.04 \mathrm{~g} / \mathrm{cm}^{3}$ & -0.00575 & \pm & 0.00017 & $\sqrt{3}$ & -0.00332 & \pm & 0.00010 & \multirow{2}{*}{519} \\
\hline & $+0.04 \mathrm{~g} / \mathrm{cm}^{3}$ & 0.00553 & \pm & 0.00016 & $\sqrt{3}$ & 0.00319 & \pm & 0.00009 & \\
\hline \multirow{2}{*}{5} & $-0.04 \mathrm{~g} / \mathrm{cm}^{3}$ & -0.00513 & \pm & 0.00016 & $\sqrt{3}$ & -0.00296 & $\perp$ & 0.00009 & \multirow{2}{*}{534} \\
\hline & $+0.04 \mathrm{~g} / \mathrm{cm}^{3}$ & 0.00473 & \pm & 0.00016 & $\sqrt{3}$ & 0.00273 & \pm & 0.00009 & \\
\hline
\end{tabular}

\footnotetext{
${ }^{a}$ Fujimoto, N., Nakano, M., Takeuchi, M., Fujisaki, S., and Yamashita, K., "Start-Up Core Physics Tests of High Temperature Engineering Test Reactor (HTTR), (II): First Criticality by an Annular Form Fuel Loading and Its Criticality Prediction Method," J. Atomic Energy Society Japan, 42(5), 458-464 (2000).
} 
NEA/NSC/DOC(2006)1

\author{
Gas Cooled (Thermal) Reactor - GCR \\ HTTR-GCR-RESR-002 \\ CRIT-REAC-RRATE
}

\title{
Impurity
}

The graphite block impurity was varied from $0-3 \mathrm{ppm}$ by weight of equivalent natural-boron content (Table 1.13 of HTTR-GCR-RESR-001), where the $3 \mathrm{ppm}$ value is 3 times the bounding limit, to determine the bounding uncertainty in $k_{\text {eff. }}$ The nominal impurity is $0.40 \mathrm{ppm}$ or $0.37 \mathrm{ppm}$ of natural boron by weight for the fuel/control blocks and reflector blocks, respectively (Table 1.27 of HTTR-GCR-RESR-001). However, characterization of the graphite first loaded into the reactor determined the equivalent boron content for IG-110 graphite to be $0.59 \mathrm{ppm} .{ }^{\mathrm{a}}$ This latter value is used in the benchmark model. Results are shown in Table 2.71.

It has been reported that the estimated air content in the graphite blocks would provide $-0.4 \% \Delta \mathrm{k} / \mathrm{k}$ to the computational model. ${ }^{\mathrm{b}}$ Reference 1 states that an uncertainty factor of $\sim 0.52 \% \Delta \mathrm{k} / \mathrm{k}$ should be used. The inclusion of air in the benchmark model did not produce a noticeable change in reactivity; this was done by modeling air, at atmospheric pressure, distributed throughout the block in the quantity equivalent to the volume fraction to the void space generated for the dowels, sockets, fuel handling position, and other miscellaneous block features. However, it is unclear exactly how much air would be entrapped within the graphite blocks. Air can be entrapped during the graphitization process or absorbed onto the graphite surface. Typically, significant contribution to the equivalent boron content in a graphite block is caused by impurities in the graphite. Methods to measure the impurity content in graphite have improved over the past several years, and the impurity content of the HTTR graphite may need to be reassessed. ${ }^{c}$

Graphite is also somewhat hydroscopic and can absorb water into its pores after fabrication. At low temperatures, the water would still be present in the graphite. Information regarding possible water content in the graphite blocks is unavailable, however.

The uncertainty in the graphite block impurity is considered all systematic with no random component.

Table 2.71. Effect of Uncertainty in IG-110 Impurity in Graphite Blocks.

\begin{tabular}{||c|c|ccc|c|ccc||}
\hline \hline Case & Deviation & $\Delta \mathrm{k}$ & \pm & $\sigma_{\Delta \mathrm{k}}$ & $\begin{array}{c}\text { Scaling } \\
\text { Factor }\end{array}$ & $\Delta \mathrm{k}_{\text {eff }}(1 \sigma)$ & \pm & $\sigma_{\Delta \mathrm{keff}}$ \\
\hline \hline \multirow{2}{*}{1} & $0 \mathrm{ppm}$ & 0.00921 & \pm & 0.00016 & $\sqrt{ } 3$ & 0.00532 & \pm & 0.00009 \\
& $3 \mathrm{ppm}$ & -0.03424 & \pm & 0.00016 & $3 \sqrt{ } 3$ & -0.00659 & \pm & 0.00003 \\
\hline \multirow{2}{*}{2} & $0 \mathrm{ppm}$ & 0.00842 & \pm & 0.00016 & $\sqrt{ } 3$ & 0.00486 & \pm & 0.00009 \\
& $3 \mathrm{ppm}$ & -0.03204 & \pm & 0.00016 & $3 \sqrt{ } 3$ & -0.00617 & \pm & 0.00003 \\
\hline \multirow{3}{*}{3} & $0 \mathrm{ppm}$ & 0.00800 & \pm & 0.00016 & $\sqrt{ } 3$ & 0.00462 & \pm & 0.00009 \\
& $3 \mathrm{ppm}$ & -0.03122 & \pm & 0.00016 & $3 \sqrt{ } 3$ & -0.00601 & \pm & 0.00003 \\
4 & $0 \mathrm{ppm}$ & 0.00727 & \pm & 0.00016 & $\sqrt{ } 3$ & 0.00420 & \pm & 0.00009 \\
& $3 \mathrm{ppm}$ & -0.02822 & \pm & 0.00017 & $3 \sqrt{ } 3$ & -0.00543 & \pm & 0.00003 \\
\hline \multirow{2}{*}{5} & $0 \mathrm{ppm}$ & 0.00848 & \pm & 0.00016 & $\sqrt{3}$ & 0.00490 & \pm & 0.00009 \\
& $3 \mathrm{ppm}$ & -0.03036 & \pm & 0.00016 & $3 \sqrt{ } 3$ & -0.00584 & \pm & 0.00003 \\
\hline
\end{tabular}

\footnotetext{
${ }^{a}$ Sumita, J., Shibata, T., Hanawa, S., Ishihara, M., Iyoku, T., and Sawa, K., "Characteristics of First Loaded IG-110 Graphite in HTTR Core," JAEA Technol 2006-048, October (2006).

${ }^{\mathrm{b}}$ Fujimoto, N., Nakano, M., Takeuchi, M., Fujisaki, S., and Yamashita, K., "Start-Up Core Physics Tests of High Temperature Engineering Test Reactor (HTTR), (II): First Criticality by an Annular Form Fuel Loading and Its Criticality Prediction Method," J. Atomic Energy Society Japan, 42(5), 458-464 (2000).
}

${ }^{c}$ Private communication with Rob Bratton at Idaho National Laboratory (November 20, 2008). 
NEA/NSC/DOC(2006)1

Gas Cooled (Thermal) Reactor - GCR

HTTR-GCR-RESR-002

CRIT-REAC-RRATE

\subsubsection{Permanent Reflectors}

\section{Density}

The PGX graphite density was varied $\pm 0.04 \mathrm{~g} / \mathrm{cm}^{3}$ from a nominal value selected as $1.73 \mathrm{~g} / \mathrm{cm}^{3}$ (ranging from 1.73 to $1.74 \mathrm{~g} / \mathrm{cm}^{3}$, Tables $1.19,1.20$, and 1.27 of HTTR-GCR-RESR-001) to determine the effective uncertainty in $k_{\text {eff. }}$ A variation of $0.03 \mathrm{~g} / \mathrm{cm}^{3}$ accounts for the PGX, and a variation of 0.01 $\mathrm{g} / \mathrm{cm}^{3}$ is assumed to encompass the variability in the volume fraction, which is not provided. The assumed uncertainty of $0.03 \mathrm{~g} / \mathrm{cm}^{3}$ for the PGX graphite encompasses the range of reported densities found throughout Section 1 of HTTR-GCR-RESR-001. Results are shown in Table 2.72. This value is treated as a bounding limit.

The total number of permanent reflector blocks used in all core configurations is 96 . For determining the random component of the uncertainty, the results in Table 2.72 would be divided by $\sqrt{ } 96$.

Table 2.72. Effect of Uncertainty in PGX Density in Permanent Reflector.

\begin{tabular}{|c|c|ccc|c|ccc||}
\hline \multirow{2}{*}{ Case } & Deviation & $\Delta \mathrm{k}$ & \pm & $\sigma_{\Delta \mathrm{k}}$ & $\begin{array}{c}\text { Scaling } \\
\text { Factor }\end{array}$ & $\Delta \mathrm{k}_{\text {eff }}(1 \sigma)$ & \pm & $\sigma_{\Delta \mathrm{keff}}$ \\
\hline \hline \multirow{2}{*}{1} & $-0.04 \mathrm{~g} / \mathrm{cm}^{3}$ & -0.00068 & \pm & 0.00017 & $\sqrt{ } 3$ & -0.00039 & \pm & 0.00010 \\
& $+0.04 \mathrm{~g} / \mathrm{cm}^{3}$ & 0.00080 & \pm & 0.00016 & $\sqrt{ } 3$ & 0.00046 & \pm & 0.00009 \\
\hline \multirow{2}{*}{2} & $-0.04 \mathrm{~g} / \mathrm{cm}^{3}$ & -0.00070 & \pm & 0.00016 & $\sqrt{ } 3$ & -0.00040 & \pm & 0.00009 \\
& $+0.04 \mathrm{~g} / \mathrm{cm}^{3}$ & 0.00098 & \pm & 0.00016 & $\sqrt{3}$ & 0.00057 & \pm & 0.00009 \\
\hline \multirow{2}{*}{3} & $-0.04 \mathrm{~g} / \mathrm{cm}^{3}$ & -0.00060 & \pm & 0.00016 & $\sqrt{3}$ & -0.00035 & \pm & 0.00009 \\
& $+0.04 \mathrm{~g} / \mathrm{cm}^{3}$ & 0.00031 & \pm & 0.00016 & $\sqrt{3}$ & 0.00018 & \pm & 0.00009 \\
\hline \multirow{3}{*}{4} & $-0.04 \mathrm{~g} / \mathrm{cm}^{3}$ & -0.00047 & \pm & 0.00017 & $\sqrt{3}$ & -0.00027 & \pm & 0.00010 \\
& $+0.04 \mathrm{~g} / \mathrm{cm}^{3}$ & 0.00033 & \pm & 0.00017 & $\sqrt{3}$ & 0.00019 & \pm & 0.00010 \\
\hline \multirow{2}{*}{5} & $-0.04 \mathrm{~g} / \mathrm{cm}^{3}$ & -0.00059 & \pm & 0.00016 & $\sqrt{3}$ & -0.00034 & \pm & 0.00009 \\
& $+0.04 \mathrm{~g} / \mathrm{cm}^{3}$ & 0.00040 & \pm & 0.00016 & $\sqrt{3}$ & 0.00023 & \pm & 0.00009 \\
\hline \hline
\end{tabular}

\section{Impurity}

The permanent reflector impurity was varied from 0-5 ppm by weight of equivalent natural-boron content (Table 1.16 of HTTR-GCR-RESR-001) to determine the bounding uncertainty in $\mathrm{k}_{\text {eff. }}$ The nominal impurity is $1.91 \mathrm{ppm}$ of natural boron by weight (Table 1.27 of HTTR-GCR-RESR-001). Results are shown in Table 2.73.

The uncertainty in the permanent reflector impurity is considered all systematic with no random component. 
NEA/NSC/DOC(2006)1

Gas Cooled (Thermal) Reactor - GCR

HTTR-GCR-RESR-002

CRIT-REAC-RRATE

Table 2.73. Effect of Uncertainty in PGX Impurity in Permanent Reflector.

\begin{tabular}{||c|c|ccc|c|ccc||}
\hline \hline Case & Deviation & $\Delta \mathrm{k}$ & \pm & $\sigma_{\Delta \mathrm{k}}$ & $\begin{array}{c}\text { Scaling } \\
\text { Factor }\end{array}$ & $\Delta \mathrm{k}_{\text {eff }}(1 \sigma)$ & \pm & $\sigma_{\Delta \mathrm{keff}}$ \\
\hline \hline \multirow{2}{*}{1} & $0 \mathrm{ppm}$ & 0.00765 & \pm & 0.00017 & $\sqrt{ } 3$ & 0.00442 & \pm & 0.00010 \\
& $5 \mathrm{ppm}$ & -0.01014 & \pm & 0.00016 & $\sqrt{ } 3$ & -0.00585 & \pm & 0.00009 \\
\hline \multirow{2}{*}{2} & $0 \mathrm{ppm}$ & 0.00656 & \pm & 0.00016 & $\sqrt{ } 3$ & 0.00379 & \pm & 0.00009 \\
& $5 \mathrm{ppm}$ & -0.00864 & \pm & 0.00017 & $\sqrt{ } 3$ & -0.00499 & \pm & 0.00010 \\
\hline \multirow{3}{*}{3} & $0 \mathrm{ppm}$ & 0.00543 & \pm & 0.00016 & $\sqrt{3}$ & 0.00314 & \pm & 0.00009 \\
& $5 \mathrm{ppm}$ & -0.00752 & \pm & 0.00017 & $\sqrt{3}$ & -0.00434 & \pm & 0.00010 \\
\hline \multirow{3}{*}{4} & $0 \mathrm{ppm}$ & 0.00296 & \pm & 0.00016 & $\sqrt{3}$ & 0.00171 & \pm & 0.00009 \\
& $5 \mathrm{ppm}$ & -0.00427 & \pm & 0.00017 & $\sqrt{3}$ & -0.00247 & \pm & 0.00010 \\
\hline \multirow{2}{*}{5} & $0 \mathrm{ppm}$ & 0.00466 & \pm & 0.00016 & $\sqrt{3}$ & 0.00269 & \pm & 0.00009 \\
& $5 \mathrm{ppm}$ & -0.00598 & \pm & 0.00016 & $\sqrt{3}$ & -0.00345 & \pm & 0.00009 \\
\hline
\end{tabular}

\subsubsection{Dummy Blocks}

\section{Density}

The IG-11 graphite density was varied $\pm 0.04 \mathrm{~g} / \mathrm{cm}^{3}$ from a nominal value of $1.75 \mathrm{~g} / \mathrm{cm}^{3}$ (Table 1.20 of HTTR-GCR-RESR-001) to determine the effective uncertainty in $k_{\text {eff. }}$ A variation of $0.03 \mathrm{~g} / \mathrm{cm}^{3}$ was selected as the density uncertainty because IG-11 graphite is essentially impure IG-110 graphite. An additional variation of $0.01 \mathrm{~g} / \mathrm{cm}^{3}$ is assumed to encompass the variability in the volume fraction caused by eliminating various features such as the dowels, sockets, and fuel handling position. Results are shown in Table 2.74. This value is treated as a bounding limit.

The total number of IG-11 graphite blocks used in the fully-loaded core is approximately zero; there are IG-11 graphite blocks in the annular core configurations. For determining the random component of the uncertainty, the results in Table 2.74 would be divided by $\sqrt{ } \mathrm{N}$, where $\mathrm{N}$ for each case is shown in Table 2.74 . 
NEA/NSC/DOC(2006)1

Gas Cooled (Thermal) Reactor - GCR

HTTR-GCR-RESR-002

CRIT-REAC-RRATE

Table 2.74. Effect of Uncertainty in IG-11 Density in Dummy Blocks.

\begin{tabular}{|c|c|c|c|c|c|c|c|c|c|}
\hline Case & Deviation & $\Delta \mathrm{k}$ & \pm & $\sigma_{\Delta k}$ & Scaling Factor & $\Delta \mathrm{k}_{\mathrm{eff}}(1 \sigma)$ & \pm & $\sigma_{\Delta \mathrm{keff}}$ & $\mathrm{N}$ \\
\hline \multirow[b]{2}{*}{1} & $-0.04 \mathrm{~g} / \mathrm{cm}^{3}$ & 0.00008 & & 0.00017 & $\sqrt{3}$ & 0.00005 & \pm & 0.00010 & \multirow{2}{*}{55} \\
\hline & $+0.04 \mathrm{~g} / \mathrm{cm}^{3}$ & -0.00050 & \pm & 0.00017 & $\sqrt{3}$ & -0.00029 & \pm & 0.00010 & \\
\hline \multirow{2}{*}{2} & $-0.04 \mathrm{~g} / \mathrm{cm}^{3}$ & 0.00054 & - & 0.00017 & $\sqrt{3}$ & 0.00031 & \pm & 0.00010 & \multirow{2}{*}{45} \\
\hline & $+0.04 \mathrm{~g} / \mathrm{cm}^{3}$ & -0.00057 & \pm & 0.00016 & $\sqrt{3}$ & -0.00033 & \pm & 0.00009 & \\
\hline \multirow{2}{*}{3} & $-0.04 \mathrm{~g} / \mathrm{cm}^{3}$ & 0.00008 & \pm & 0.00016 & $\sqrt{3}$ & 0.00005 & \pm & 0.00009 & \multirow{2}{*}{30} \\
\hline & $+0.04 \mathrm{~g} / \mathrm{cm}^{3}$ & -0.00046 & 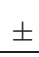 & 0.00017 & $\sqrt{3}$ & -0.00027 & \pm & 0.00010 & \\
\hline \multirow{2}{*}{4} & $-0.04 \mathrm{~g} / \mathrm{cm}^{3}$ & 0.00016 & & 0.00017 & $\sqrt{3}$ & 0.00009 & \pm & 0.00010 & \multirow{2}{*}{30} \\
\hline & $+0.04 \mathrm{~g} / \mathrm{cm}^{3}$ & -0.00051 & + & 0.00017 & $\sqrt{3}$ & -0.00029 & \pm & 0.00010 & \\
\hline & $-0.04 \mathrm{~g} / \mathrm{cm}^{3}$ & -0.00016 & \pm & 0.00016 & $\sqrt{3}$ & 0.00009 & \pm & 0.00009 & \multirow{2}{*}{15} \\
\hline & $+0.04 \mathrm{~g} / \mathrm{cm}^{3}$ & -0.00011 & \pm & 0.00016 & $\sqrt{3}$ & -0.00006 & \pm & 0.00009 & \\
\hline
\end{tabular}

\section{Impurity}

The graphite block impurity was varied from $0-5 \mathrm{ppm}$ by weight of equivalent natural-boron content to determine the bounding uncertainty in $\mathrm{k}_{\mathrm{eff}}$. The nominal impurity is $3.1 \mathrm{ppm}$ natural boron by weight (Ref 1, p. 314). The dummy blocks in the HTTR are created from the same graphite material, IG-11, as the reflector graphite in the HTR-10 reactor. ${ }^{\mathrm{ab}}$ The maximum impurity content was derived from the reflector graphite in the HTR-10 reactor (HTR10-GCR-RESR-001), which has a nominal boron concentration of $4.8366 \pm 0.09673 \mathrm{ppm}( \pm 2 \%)$. Results are shown in Table 2.75.

The uncertainty in the graphite block impurity is considered all systematic with no random component.

\footnotetext{
${ }^{a}$ L. Xiaowei, R. Jean-Charles, and Y. Suyuan, "Effect of Temperature on Graphite Oxidation Behavior," Nucl. Eng. Des., 227: 273-280 (2004).

${ }^{\mathrm{b}}$ X. Luo, J-C. Robin, and S. Yu, "Comparison of Oxidation Behaviors of Different Grades of Nuclear Graphite," Nucl. Sci. Eng., 151, 121-127 (2005).
}

Revision: 0 
NEA/NSC/DOC(2006)1

Gas Cooled (Thermal) Reactor - GCR

HTTR-GCR-RESR-002

CRIT-REAC-RRATE

Table 2.75. Effect of Uncertainty in IG-11 Impurity in Dummy Blocks.

\begin{tabular}{|c|c|ccc|c|ccc||}
\hline \hline Case & Deviation & $\Delta \mathrm{k}$ & \pm & $\sigma_{\Delta \mathrm{k}}$ & $\begin{array}{c}\text { Scaling } \\
\text { Factor }\end{array}$ & $\Delta \mathrm{k}_{\text {eff }}(1 \sigma)$ & \pm & $\sigma_{\Delta \mathrm{keff}}$ \\
\hline \hline \multirow{2}{*}{1} & $0 \mathrm{ppm}$ & 0.01219 & \pm & 0.00017 & $\sqrt{ } 3$ & 0.00704 & \pm & 0.00010 \\
& $5 \mathrm{ppm}$ & -0.00671 & \pm & 0.00017 & $\sqrt{ } 3$ & -0.00387 & \pm & 0.00010 \\
\hline \multirow{2}{*}{2} & $0 \mathrm{ppm}$ & 0.01278 & \pm & 0.00016 & $\sqrt{ } 3$ & 0.00738 & \pm & 0.00009 \\
& $5 \mathrm{ppm}$ & -0.00674 & \pm & 0.00017 & $\sqrt{ } 3$ & -0.00389 & \pm & 0.00010 \\
\hline \multirow{3}{*}{3} & $0 \mathrm{ppm}$ & 0.00797 & \pm & 0.00017 & $\sqrt{ } 3$ & 0.00460 & \pm & 0.00010 \\
& $5 \mathrm{ppm}$ & -0.00457 & \pm & 0.00017 & $\sqrt{3}$ & -0.00264 & \pm & 0.00010 \\
\hline \multirow{3}{*}{4} & $0 \mathrm{ppm}$ & 0.01037 & \pm & 0.00017 & $\sqrt{ } 3$ & 0.00599 & \pm & 0.00010 \\
& $5 \mathrm{ppm}$ & -0.00580 & \pm & 0.00017 & $\sqrt{3}$ & -0.00335 & \pm & 0.00010 \\
\hline \multirow{2}{*}{5} & $0 \mathrm{ppm}$ & 0.00376 & \pm & 0.00016 & $\sqrt{ } 3$ & 0.00217 & \pm & 0.00009 \\
& $5 \mathrm{ppm}$ & -0.00215 & \pm & 0.00016 & $\sqrt{3}$ & -0.00124 & \pm & 0.00009 \\
\hline
\end{tabular}

\section{Block Type}

There are two types of dummy blocks, one with a hole pattern similar to that of a control block, and the other with three holes but of smaller diameter (Ref. 2, p. 14). Elsewhere the source of eight types of dummy blocks are specified (most likely having one of the two hole patterns). ${ }^{\mathrm{a}}$ Because the true dimensions of the smaller hole design is unknown, the dummy blocks will all be modeled with the holes of the control blocks but an additional uncertainty will be assessed to account for this discrepancy. This uncertainty will be determined by completely filling the holes of the dummy blocks with IG-11 graphite material as a bounding limit (i.e., maximizing the amount of graphite material mass) representing the minimum dimensions of three infinitely thin holes in the dummy blocks. The volume fraction of the simulated control channels in a dummy blocks is approximately $31.76 \%$, representing an increase in graphite mass of $36 \mathrm{~kg}$ per block when the holes are completely filled (based on a graphite density of $1.75 \mathrm{~g} / \mathrm{cm}^{3}$ with an $0.005 \%$ reduction for void volume). Results are shown in Table 2.76 .

There are no IG-11 graphite blocks used in the fully-loaded core; there are IG-11 graphite blocks in the annular core configurations. For determining the random component of the uncertainty, the results in Table 2.76 would be divided by $\sqrt{ } \mathrm{N}$, where $\mathrm{N}$ for each case is shown in Table 2.76 .

\footnotetext{
${ }^{a}$ N. Fujimoto, N. Nojiri, and K. Yamashita, "HTTR's Benchmark Calculation of Start-Up Core Physics Tests," Report of the $3^{\text {rd }}$ Research Coordination Meeting on the CRP, IAEA, Oarai, Japan, March 12-16 (2001).
} 
NEA/NSC/DOC(2006)1

Gas Cooled (Thermal) Reactor - GCR

HTTR-GCR-RESR-002

CRIT-REAC-RRATE

Table 2.76. Effect of Uncertainty in Dummy Blocks Type.

\begin{tabular}{||c|c|ccc|c|ccc|c||}
\hline \hline Case & Deviation & $\Delta \mathrm{k}$ & \pm & $\sigma_{\Delta \mathrm{k}}$ & $\begin{array}{c}\text { Scaling } \\
\text { Factor }\end{array}$ & $\Delta \mathrm{k}_{\text {eff }}(1 \sigma)$ & \pm & $\sigma_{\Delta \mathrm{keff}}$ & $\mathrm{N}$ \\
\hline \hline 1 & Filled Holes & -0.00058 & \pm & 0.00017 & $2 \sqrt{ } 3$ & -0.00017 & \pm & 0.00005 & 55 \\
\hline 2 & Filled Holes & -0.00507 & \pm & 0.00017 & $2 \sqrt{3}$ & -0.00146 & \pm & 0.00005 & 45 \\
\hline 3 & Filled Holes & -0.00200 & \pm & 0.00017 & $2 \sqrt{3}$ & -0.00058 & \pm & 0.00005 & 30 \\
\hline 4 & Filled Holes & -0.00893 & \pm & 0.00017 & $2 \sqrt{3}$ & -0.00258 & \pm & 0.00005 & 30 \\
\hline 5 & Filled Holes & 0.00234 & \pm & 0.00016 & $2 \sqrt{ } 3$ & 0.00068 & \pm & 0.00005 & 15 \\
\hline \hline
\end{tabular}

\subsubsection{Helium Coolant}

\section{Density}

The density of helium gas was evaluated using the ideal gas law, PV=nRT, at a pressure of $1 \mathrm{~atm}$ and temperature of $25^{\circ} \mathrm{C}$. The helium coolant was modeled with an atom density of $2.4616 \times 10^{-4}$ atoms $/ \mathrm{b}$ $\mathrm{cm}$ (mass density of $\left.1.6361 \times 10^{-4} \mathrm{~g} / \mathrm{cm}^{3}\right)$.

The helium density was varied approximately $\pm 35 \%(10 \times$ bounding limit $)$ to determine the effective uncertainty in $\mathrm{k}_{\text {eff. }}$ Results are shown in Table 2.77 .

The effect of neglecting the helium content and replacing it with an empty void was also performed. The effective change in change in $\mathrm{k}_{\text {eff }}$ was determined. Results are shown in Table 2.77.

The uncertainty in the helium density is considered all systematic with no random component. 
NEA/NSC/DOC(2006)1

Gas Cooled (Thermal) Reactor - GCR

HTTR-GCR-RESR-002

CRIT-REAC-RRATE

Table 2.77. Effect of Uncertainty in Helium Coolant Density.

\begin{tabular}{|c|c|c|c|c|c|c|c|c|}
\hline Case & Deviation & $\Delta \mathrm{k}$ & \pm & $\sigma_{\Delta \mathrm{k}}$ & $\begin{array}{l}\text { Scaling } \\
\text { Factor }\end{array}$ & $\Delta \mathrm{k}_{\mathrm{eff}}(1 \sigma)$ & \pm & $\sigma_{\Delta \mathrm{keff}}$ \\
\hline \multirow{3}{*}{1} & void & -0.00006 & \pm & 0.00016 & 1 & -0.00006 & \pm & 0.00016 \\
\hline & $-35 \%(10 \sigma)$ & -0.00007 & \pm & 0.00017 & $10 \sqrt{3}$ & 0.00000 & \pm & 0.00001 \\
\hline & $+35 \%(10 \sigma)$ & 0.00005 & \pm & 0.00017 & $10 \sqrt{3}$ & 0.00000 & \pm & 0.00001 \\
\hline \multirow{3}{*}{2} & void & 0.00014 & \pm & 0.00017 & 1 & 0.00014 & \pm & 0.00017 \\
\hline & $-35 \%(10 \sigma)$ & 0.00004 & \pm & 0.00017 & $10 \sqrt{3}$ & 0.00000 & \pm & 0.00001 \\
\hline & $+35 \%(10 \sigma)$ & 0.00021 & \pm & 0.00016 & $10 \sqrt{3}$ & 0.00001 & \pm & 0.00001 \\
\hline \multirow{3}{*}{3} & void & -0.00014 & \pm & 0.00016 & 1 & -0.00014 & \pm & 0.00016 \\
\hline & $-35 \%(10 \sigma)$ & -0.00003 & \pm & 0.00016 & $10 \sqrt{3}$ & 0.00000 & \pm & 0.00000 \\
\hline & $+35 \%(10 \sigma)$ & 0.00004 & $\perp$ & 0.00017 & $10 \sqrt{3}$ & 0.00000 & \pm & 0.00000 \\
\hline \multirow{3}{*}{4} & void & -0.00005 & \pm & 0.00016 & 1 & -0.00005 & \pm & 0.00016 \\
\hline & $-35 \%(10 \sigma)$ & -0.00022 & \pm & 0.00017 & $10 \sqrt{3}$ & -0.00001 & \pm & 0.00001 \\
\hline & $+35 \%(10 \sigma)$ & -0.00016 & \pm & 0.00017 & $10 \sqrt{3}$ & -0.00001 & \pm & 0.00001 \\
\hline \multirow{3}{*}{5} & void & 0.00018 & + & 0.00016 & 1 & 0.00018 & \pm & 0.00016 \\
\hline & $-35 \%(10 \sigma)$ & -0.00010 & \pm & 0.00016 & $10 \sqrt{3}$ & -0.00001 & \pm & 0.00001 \\
\hline & $+35 \%(10 \sigma)$ & 0.00001 & \pm & 0.00016 & $10 \sqrt{3}$ & 0.00000 & \pm & 0.00001 \\
\hline
\end{tabular}

\section{Impurity}

The upper impurity limit in the helium coolant was included in the model to determine its effect upon the uncertainty in $\mathrm{k}_{\mathrm{eff}}$. Upper concentration limits from Table 1.10 of HTTR-GCR-RESR-001 were included although the impurity content in helium at room temperature would be considerably less (and practically nonexistent); therefore it will be treated as a bounding limit. The bounding limit was multiplied by 10 for each component in order to assess the uncertainty, but the effects were still negligible. Results are shown in Table 2.78 .

The uncertainty in the helium impurity is considered all systematic with no random component.

Table 2.78. Effect of Uncertainty in Helium Coolant Impurity.

\begin{tabular}{||c|c|ccc|c|ccc||}
\hline \hline Case & Deviation & $\Delta \mathrm{k}$ & \pm & $\sigma_{\Delta \mathrm{k}}$ & $\begin{array}{c}\text { Scaling } \\
\text { Factor }\end{array}$ & $\Delta \mathrm{k}_{\text {eff }}(1 \sigma)$ & \pm & $\sigma_{\Delta \mathrm{keff}}$ \\
\hline \hline 1 & Added Impurities $(\times 10)$ & -0.00004 & \pm & 0.00017 & $20 \sqrt{3}$ & 0.00000 & \pm & 0.00000 \\
2 & Added Impurities $(\times 10)$ & 0.00024 & \pm & 0.00017 & $20 \sqrt{3}$ & 0.00001 & \pm & 0.00000 \\
3 & Added Impurities $(\times 10)$ & 0.00011 & \pm & 0.00016 & $20 \sqrt{ } 3$ & 0.00000 & \pm & 0.00000 \\
4 & Added Impurities $(\times 10)$ & 0.00003 & \pm & 0.00017 & $20 \sqrt{3}$ & 0.00000 & \pm & 0.00000 \\
5 & Added Impurities $(\times 10)$ & 0.00005 & \pm & 0.00016 & $20 \sqrt{3}$ & 0.00000 & \pm & 0.00000 \\
\hline
\end{tabular}


NEA/NSC/DOC(2006)1

Gas Cooled (Thermal) Reactor - GCR

HTTR-GCR-RESR-002

CRIT-REAC-RRATE

\subsubsection{Additional Analyses}

\subsubsection{Room Return}

Insufficient information is available to model and evaluate the uncertainties and biases related to any room return effects in the HTTR. Shielding plugs, plates, and blocks are incorporated within the HTTR vessel and would considerably reduce room return effects from the surrounding reactor vessel, reactor internals, and HTTR infrastructure and facility due to the content of sintered $\mathrm{B}_{4} \mathrm{C} / \mathrm{C}$ neutron absorber.

A conservative analysis of the HTTR benchmark model of the fully-loaded core (HTTR-GCR-RESR-001) surrounded by shielding material, steel, and concrete provided an insignificant effect because the slight increase in the effective multiplication factor was well below the range of statistical uncertainty for the analysis. Actual room return effects would be assumed quite negligible.

\subsubsection{TRISO Particle Placement}

Currently MCNP has a limited capability for modeling stochastic geometries. The HTTR core is unique in the fact that it has 12 different enrichments throughout the core. MCNP could effectively model randomness for only two types of TRISO particles at once because the URAN card used to create stochastic models has a maximum limit of two universes per model. A comparison of using the URAN analysis with multiple input decks to represent pairs of enriched TRISO particles was performed for the fully-loaded core configuration (HTTR-GCR-RESR-001) and shown to provide equivalent results to the approach performed below.

To approximate the bounding uncertainty in the effects of random TRISO placement, the fuel compacts were modeled with a uniform cell lattice of TRISO particles, which effectively creates partial particles along the compact borders. Figures 2.6 and 2.7 show a cross section of the fuel compacts with ordered and uniformly-filled TRISO distributions, respectively. The effective difference in the multiplication factor is shown in Table 2.79. The 30 vol. \% packing fraction and uranium mass per fuel rod is conserved in this comparison. In order to conserve the uranium mass, the volume was estimated for the uranium kernels along the edges of the fuel compacts (using linear approximation of the cylindrical surface and spherical dome formulas) ${ }^{\mathrm{a}}$ to determine the approximate fuel content in each compact. Then the uniform cell lattice was adjusted such that the modeled fuel content matched the reported HTTR value. The reduction in resonance shielding effects in partial particles (i.e. fuel kernels not completely surrounded by graphite coatings) along the edges is not considered in this analysis. There is a slight mass uncertainty that is unaccounted for in this approximation. Because this is a bounding application, the results in Table 2.79 need to be divided by the square-root of three.

An analysis performed at the University of Michigan to look at the effects of explicitly modeling particle fuel in a very-high temperature gas-cooled reactor found that in a full core model, the effect of modeling with a uniform lattice and clipped TRISO particles would have a reduced $\mathrm{k}_{\text {eff }}$ of approximately $0.1 \%$ compared to a heterogeneous core design containing only complete TRISO particles. ${ }^{b}$ The results shown in Table 2.79 are approximately $0.1 \%$ for the fully-loaded core configuration and slightly less for these annular configurations. The values calculated in Table 2.79 are used to represent the uncertainty in random TRISO particle placement in the HTTR. This uncertainty is treated as $100 \%$ systematic to capture the complete uncertainty in random particle placement.

A driver for this phenomenon might be explained by the Dancoff-Ginsberg factor, where in a lattice, the closer the lumps of fissionable material are to each other, the greater the shadowing effect and the smaller

\footnotetext{
a "Spherical and Ellipsoid Dome Formulas," Monolithic Dome Institute, Italy, Texas (2001).

${ }^{b}$ W. Ji, J. L. Conlin, W. R. Martin, J. C. Lee, and F. B. Brown, "Explicit Modeling of Particle Fuel for the VeryHigh Temperature Gas-Cooled Reactor,” Trans. Am. Nucl. Soc., 92 (June 2005). 
NEA/NSC/DOC(2006)1

Gas Cooled (Thermal) Reactor - GCR

HTTR-GCR-RESR-002

CRIT-REAC-RRATE

the resonance integral for the interacting lattice, which results in an increase in the non-leakage probability and net increase in $\mathrm{k}_{\mathrm{eff}} \mathrm{a}^{\mathrm{a}}$

A competing argument is that self-shielding and shadowing effects are quite negligible ( ${ }^{\text {nd }}$-order effect) and that small variations in fuel conservation actually lead to the difference in the calculated value of $\mathrm{k}_{\mathrm{eff}}{ }^{\text {bc }}$ Further discussion of the relevance of fuel particle parameters upon the reactivity of the system can be read elsewhere. ${ }^{\mathrm{d}}$ It remains up to the user's discretion to assess whether and how an uncertainty might apply to the modeling of this reactor system.

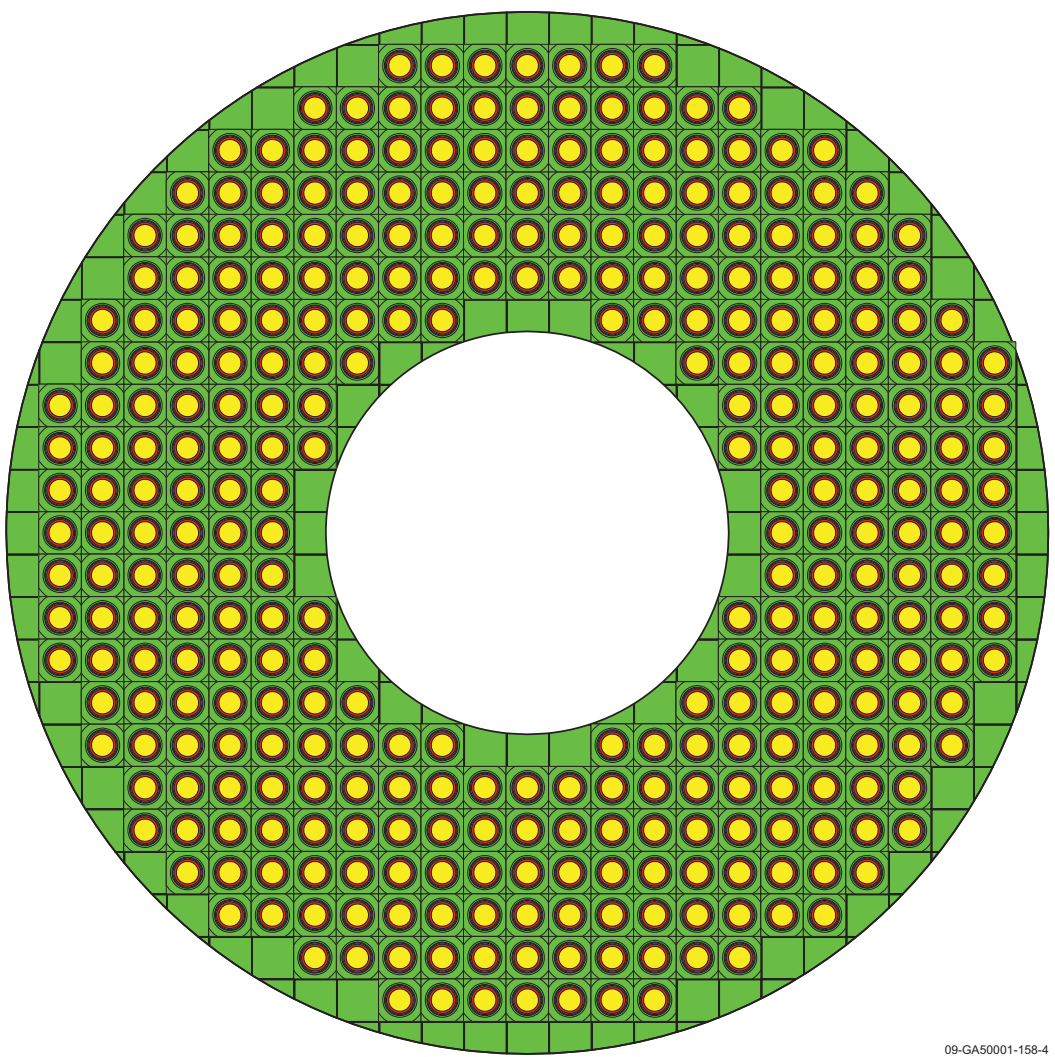

Figure 2.6. MCNP Ordered TRISO Lattice within the Fuel Compacts.

${ }^{a}$ J. R. Lamarsh, Introduction to Nuclear Reactor Theory, Addison-Wesley Publishing Company, Reading, Massachusetts, pp. 399-400 (1966).

${ }^{\mathrm{b}}$ Personal communication between Luka Snoj and Forrest Brown from Los Alamos National Laboratory (November 20, 2008).

c F. B. Brown, "Monte Carlo Advances \& Challenges," Proc. Frederic Joliot and Otto Hahn Summer School 2005, Karlsruhe, Germany, August 24 - September 2 (2005).

d L. Snoj and M. Ravnik, "Effect of Fuel Particles' Size and Position Variations on Multiplication Factor in PebbleBed Nuclear Reactors," Kerntechnik, 72 (2007). 
NEA/NSC/DOC(2006)1

Gas Cooled (Thermal) Reactor - GCR

HTTR-GCR-RESR-002

CRIT-REAC-RRATE

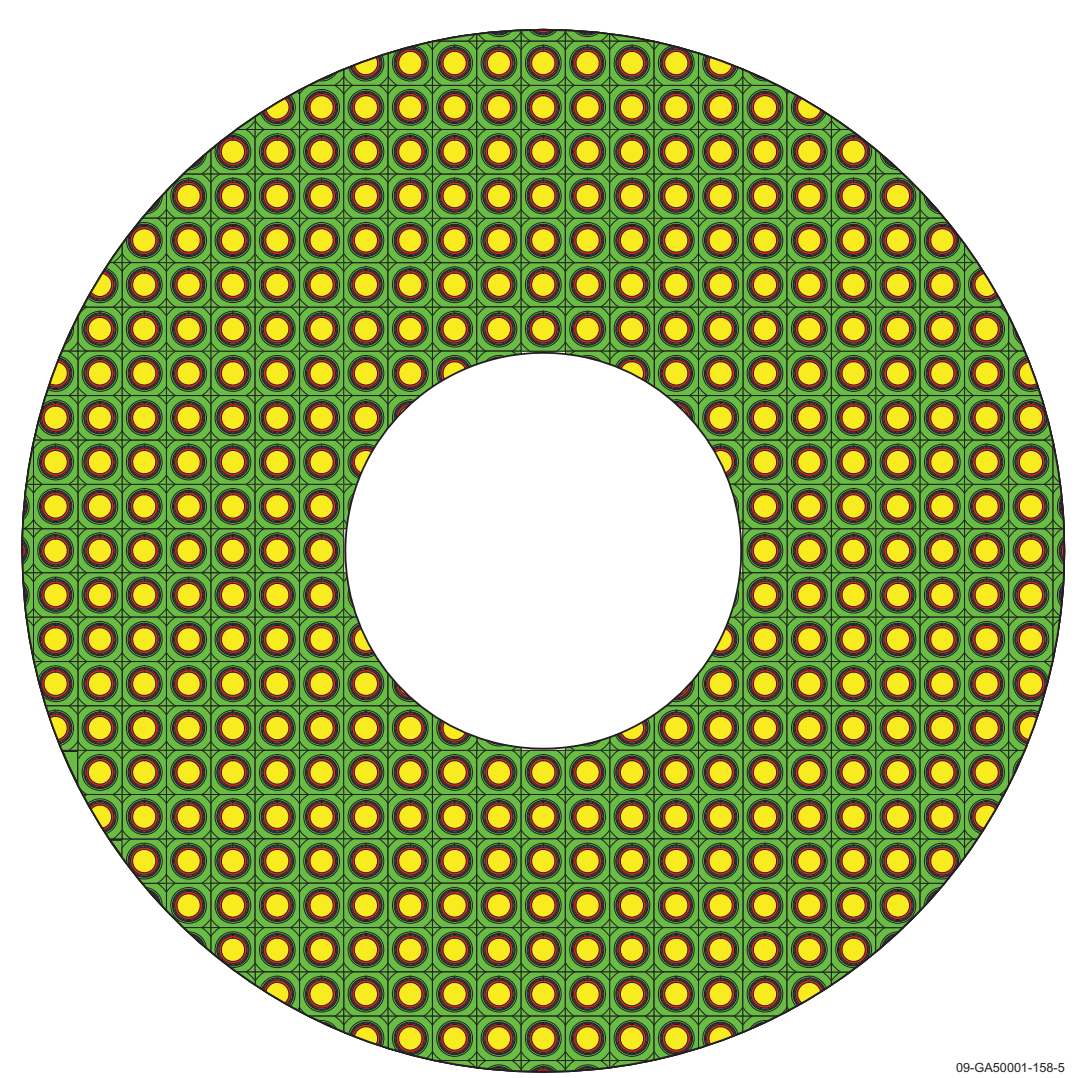

Figure 2.7. MCNP Uniformly-Filled TRISO Lattice within the Fuel Compacts.

Table 2.79. Comparison of Uniform and Organized TRISO Fill in Fuel Compacts.

\begin{tabular}{|c|c|c|c|c|c|c|c|c|c|c|c|c|}
\hline \multirow[t]{2}{*}{ Case } & \multicolumn{3}{|c|}{$\begin{array}{c}\text { Organized } \\
\text { Distribution } \\
\text { (Figure 2.6) }\end{array}$} & \multicolumn{3}{|c|}{$\begin{array}{l}\text { Uniform Fill } \\
\text { (Figure 2.7) }\end{array}$} & \multicolumn{3}{|c|}{ Bounding Difference } & \multicolumn{3}{|c|}{$1 \sigma$ Uncertainty } \\
\hline & $\mathrm{k}_{\mathrm{eff}}$ & \pm & $\sigma_{\mathrm{k}}$ & $\mathrm{k}_{\mathrm{eff}}$ & \pm & $\sigma_{\mathrm{k}}$ & $\Delta \mathrm{k}$ & \pm & $\sigma_{\Delta \mathrm{k}}$ & $\Delta \mathrm{k}$ & \pm & $\sigma_{\Delta \mathrm{k}}$ \\
\hline 1 & 1.03123 & \pm & 0.00012 & 1.03087 & \pm & 0.00011 & 0.00036 & \pm & 0.00016 & 0.00021 & \pm & 0.00009 \\
\hline 2 & 1.03290 & \pm & 0.00012 & 1.03233 & $\perp$ & 0.00011 & 0.00057 & \pm & 0.00016 & 0.00033 & \pm & 0.00009 \\
\hline 3 & 1.02847 & \pm & 0.00012 & 1.02758 & \pm & 0.00012 & 0.00089 & \pm & 0.00017 & 0.00051 & \pm & 0.00010 \\
\hline 4 & 1.03193 & \pm & 0.00012 & 1.03101 & \pm & 0.00012 & 0.00092 & \pm & 0.00017 & 0.00053 & \pm & 0.00009 \\
\hline 5 & 1.02516 & \pm & 0.00011 & 1.02399 & \pm & 0.00012 & 0.00117 & \pm & 0.00016 & 0.00068 & \pm & 0.00009 \\
\hline
\end{tabular}


NEA/NSC/DOC(2006)1

\section{Gas Cooled (Thermal) Reactor - GCR \\ HTTR-GCR-RESR-002 \\ CRIT-REAC-RRATE}

\subsubsection{Block Stack Alignment}

As discussed in the previous section, MCNP has a limited capability for modeling stochastic geometries for systems with multiple unique components. Whereas the HTTR core model is comprised of 61 columns each containing 9 bricks, a comprehensive analysis of the stochastic nature of block stacking could not be easily evaluated.

\subsubsection{MCNP Random Number Generation}

The random number seed was changed for the benchmark model and compared with the original to determine the uncertainty in utilizing Monte Carlo analysis methods. Results are shown in Table 2.80. The uncertainty in the average is the standard deviation of the six averaged eigenvalues.

Table 2.80. Analysis of the Effect of Random Number Generation in MCNP.

\begin{tabular}{||c|ccc|ccc|ccc||}
\hline \multirow{2}{*}{ Seed } & \multicolumn{3}{|c|}{ Case 1 } & \multicolumn{3}{c|}{ Case 2 } & \multicolumn{3}{c||}{ Case 3 } \\
& $\mathrm{k}_{\text {eff }}$ & \pm & $\sigma_{\text {keff }}$ & $\mathrm{k}_{\text {eff }}$ & \pm & $\sigma_{\text {keff }}$ & $\mathrm{k}_{\text {eff }}$ & \pm & $\sigma_{\text {keff }}$ \\
\hline 123456787 & 1.03121 & \pm & 0.00012 & 1.03304 & \pm & 0.00012 & 1.02831 & \pm & 0.00012 \\
9876543279 & 1.03145 & \pm & 0.00012 & 1.03292 & \pm & 0.00012 & 1.02850 & \pm & 0.00012 \\
198765432799 & 1.03111 & \pm & 0.00011 & 1.03293 & \pm & 0.00012 & 1.02841 & \pm & 0.00011 \\
17623486105893 & 1.03131 & \pm & 0.00011 & 1.03308 & \pm & 0.00012 & 1.02847 & \pm & 0.00012 \\
$19073486328125^{(a)}$ & 1.03123 & \pm & 0.00012 & 1.03290 & \pm & 0.00012 & 1.02847 & \pm & 0.00012 \\
32160231045432797 & 1.03107 & \pm & 0.00011 & 1.03291 & \pm & 0.00011 & 1.02844 & \pm & 0.00012 \\
\hline \hline Average & 1.03123 & \pm & 0.00014 & 1.03296 & \pm & 0.00008 & 1.02843 & \pm & 0.00007 \\
\hline \hline
\end{tabular}

Table 2.80 (cont'd.). Analysis of the Effect of Random Number Generation in MCNP.

\begin{tabular}{|c|c|c|c|c|c|c|}
\hline \multirow{2}{*}{ Seed } & \multicolumn{3}{|c|}{ Case 4} & \multicolumn{3}{|c|}{ Case 5} \\
\hline & $k_{\text {eff }}$ & \pm & $\sigma_{\text {keff }}$ & $\mathrm{k}_{\mathrm{eff}}$ & \pm & $\sigma_{\text {keff }}$ \\
\hline 123456787 & 1.03186 & \pm & 0.00012 & 1.02511 & \pm & 0.00011 \\
\hline 9876543279 & 1.03169 & \pm & 0.00012 & 1.02495 & \pm & 0.00011 \\
\hline 198765432799 & 1.03177 & \pm & 0.00012 & 1.02539 & \pm & 0.00012 \\
\hline 17623486105893 & 1.03192 & \pm & 0.00012 & 1.02513 & \pm & 0.00012 \\
\hline $19073486328125^{(\mathrm{a})}$ & 1.03193 & \pm & 0.00012 & 1.02516 & \pm & 0.00011 \\
\hline 32160231045432797 & 1.03204 & \pm & 0.00012 & 1.02536 & \pm & 0.00011 \\
\hline Average & 1.03187 & \pm & 0.00012 & 1.02518 & \pm & 0.00017 \\
\hline
\end{tabular}

(a) Primary seed value for evaluation.

\subsubsection{Simplification Biases and Uncertainties}

Whereas insufficient information is publicly available, a comprehensive analysis of simplification biases and their respective uncertainties could not be appropriately assessed. Currently only an approximate bias for the instrumentation components in the reactor has been assessed (Section 2.1.2.6). As additional 
NEA/NSC/DOC(2006)1

\section{Gas Cooled (Thermal) Reactor - GCR \\ HTTR-GCR-RESR-002 \\ CRIT-REAC-RRATE}

information becomes available, highly detailed and simplified benchmark models can be generated and their biases can be adequately determined.

\subsubsection{Systematic Biases and Uncertainties}

There was no information regarding systematic biases or uncertainties publicly available for these experiments. Previous efforts of the Japanese in analyzing the 19-fuel-column core (Case 1) obtained an analytical excess reactivity of $2.7 \% \Delta \mathrm{k} / \mathrm{k}$, with an estimated Monte Carlo calculation overestimate of 1.2 $\% \Delta \mathrm{k} / \mathrm{k}^{\mathrm{a}}{ }^{\mathrm{a}}$ Additional information would be necessary to completely verify published results to generate an analytical bias for MCNP.

As discussed at the beginning of Section 2, all uncertainties are treated as $25 \%$ systematic, with no reduction in uncertainty due to the multiplicity of core components, and as $75 \%$ random.

\subsubsection{Analysis of HTTR Uranium Content}

The parameters (dimensions, density, etc.) of the TRISO particles fabricated during the manufacturing process are very normally distributed, except for any defective particles. The fuel content, or mass, is the most well-known specification and measured with the highest accuracy.

Because of the overspecification of the TRISO particles in Table 1.14 of HTTR-GCR-RESR-001 and the correlation of uranium kernel diameter, density, packing fraction, and mass, the effect of the uncertainties in the kernel diameter, density, and packing fraction are not included in the total uncertainty as separate entities. The uranium content of the fuel rods of $188.58 \pm 5.66 \mathrm{~g}$ (Table $1.14 \mathrm{of}$ HTTR-GCR-RESR-001) is the parameter most likely known with the greatest accuracy. Therefore, the diameter of the kernels will be fixed at $600 \mu \mathrm{m}$, and the density will be varied $\pm 0.32 \mathrm{~g} / \mathrm{cm}^{3}$ from a nominal value of $10.39 \mathrm{~g} / \mathrm{cm}^{3}$ to determine the effective uncertainty in $\mathrm{k}_{\mathrm{eff}}$ due to the uranium mass uncertainty. Results are shown in Table 2.81. This value is treated as a bounding limit.

The total number of fuel rods used in the fully-loaded core is approximately 4,770. For determining the random component of the uncertainty, the results in Table 2.81 would be divided by $\sqrt{ } \mathrm{N}$.

\footnotetext{
${ }^{a}$ Fujimoto, N., Nakano, M., Takeuchi, M., Fujisaki, S., and Yamashita, K., "Start-Up Core Physics Tests of High Temperature Engineering Test Reactor (HTTR), (II): First Criticality by an Annular Form Fuel Loading and Its Criticality Prediction Method," J. Atomic Energy Society Japan, 42(5), 458-464 (2000).

${ }^{b}$ Personal communication with David Petti from the Idaho National Laboratory (September 28, 2009).
} 
NEA/NSC/DOC(2006)1

Gas Cooled (Thermal) Reactor - GCR

HTTR-GCR-RESR-002

CRIT-REAC-RRATE

Table 2.81. Effect of Uncertainty in Uranium Mass.

\begin{tabular}{|c|c|c|c|c|c|c|c|c|c|}
\hline Case & Deviation & $\Delta \mathrm{k}$ & \pm & $\sigma_{\Delta \mathrm{k}}$ & $\begin{array}{l}\text { Scaling } \\
\text { Factor }\end{array}$ & $\Delta \mathrm{k}_{\mathrm{eff}}(1 \sigma)$ & \pm & $\sigma_{\Delta \mathrm{keff}}$ & $\mathrm{N}$ \\
\hline \multirow[b]{2}{*}{1} & $-0.32 \mathrm{~g} / \mathrm{cm}^{3}$ & & \pm & 0.0 & $\sqrt{3}$ & & \pm & & \multirow{2}{*}{2,955} \\
\hline & $+0.32 \mathrm{~g} / \mathrm{cm}^{3}$ & 0.00482 & \pm & 0.00016 & $\sqrt{3}$ & 0.00278 & \pm & 0.00009 & \\
\hline \multirow[b]{2}{*}{2} & $-0.32 \mathrm{~g} / \mathrm{cm}^{3}$ & -0.00487 & \pm & 0.00017 & $\sqrt{3}$ & -0.00281 & \pm & 0.00010 & \multirow{2}{*}{3,285} \\
\hline & $+0.32 \mathrm{~g} / \mathrm{cm}^{3}$ & 0.00511 & $\perp$ & 17 & $\sqrt{3}$ & 0 . & \pm & 10 & \\
\hline \multirow[b]{2}{*}{3} & $-0.32 \mathrm{~g} / \mathrm{cm}^{3}$ & -0.00535 & - & 0.00017 & $\sqrt{3}$ & -0.00309 & \pm & 0.00010 & \multirow{2}{*}{3,780} \\
\hline & $+0.32 \mathrm{~g} / \mathrm{cm}^{3}$ & 0.00 & $I$ & 17 & $\sqrt{3}$ & 0. & \pm & 10 & \\
\hline \multirow[b]{2}{*}{4} & $-0.32 \mathrm{~g} / \mathrm{cm}^{3}$ & -0.00520 & \pm & 0.00017 & $\sqrt{3}$ & -0.00300 & \pm & 0.00010 & \multirow{2}{*}{3,780} \\
\hline & $+0.32 \mathrm{~g} / \mathrm{cm}^{3}$ & 0.00498 & + & 0.00017 & $\sqrt{3}$ & 0.00288 & \pm & 0.00010 & \\
\hline & $-0.32 \mathrm{~g} / \mathrm{cm}^{3}$ & -0.00519 & \pm & 0.00016 & $\sqrt{3}$ & -0.00300 & \pm & 0.00009 & \multirow{2}{*}{4,275} \\
\hline & $+0.32 \mathrm{~g} / \mathrm{cm}^{3}$ & 0.00507 & \pm & 0.00016 & $\sqrt{3}$ & 0.00293 & \pm & 0.00009 & \\
\hline
\end{tabular}

\subsubsection{Total Experimental Uncertainty}

A compilation of the total evaluated uncertainty in the HTTR model is shown in Tables 2.82 through 2.86 for configurations 1 through 5, respectively. As discussed earlier, each of the evaluated uncertainties is divided into a systematic component $(25 \%)$ and random component $(75 \%)$, where appropriate. The random component is then divided by the square-root of the number of random objects reported in the subsection containing the calculated base uncertainty values. The root-mean square of each subcomponent is taken to determine the uncertainty in either the random or systematic components of the total evaluated uncertainty. The total evaluated uncertainty is then the root-mean square of the random and systematic uncertainties.

Uncertainties less than 0.00001 are reported as negligible (neg). When calculated uncertainties in $\Delta \mathrm{k}_{\text {eff }}$ are less than their statistical uncertainties, the statistical uncertainties are used in the calculation of the total uncertainty. Table listings where calculations were not performed or otherwise not available are labeled with 'NA'. For uncertainties where a random component is not applicable, the uncertainty is denoted with '--'.

The most significant contributions to the overall uncertainty from the systematic uncertainties include the impurities in the IG-110 graphite blocks, PGX graphite reflector blocks, and IG-11 graphite dummy blocks. All uncertainties providing at least $0.1 \% \Delta \mathrm{k}_{\text {eff }}$ are highlighted in gray in Tables 2.82 through 2.86. All of the random uncertainties are less than $0.1 \% \Delta \mathrm{k}_{\text {eff. }}$. The overall uncertainty is less than $1 \%$ $\Delta \mathrm{k}_{\text {eff }}$ (except for Cases 1 and 2); it is expected that the total uncertainty will be reduced as additional parameters that characterize the HTTR are obtained. 
NEA/NSC/DOC(2006)1

Gas Cooled (Thermal) Reactor - GCR

HTTR-GCR-RESR-002

CRIT-REAC-RRATE

Table 2.82. Total Experimental Uncertainty (Case 1).

\begin{tabular}{|c|c|c|c|c|}
\hline \multirow{2}{*}{ Varied Parameter } & \multicolumn{2}{|c|}{ Systematic Uncertainty } & \multicolumn{2}{|c|}{ Random Uncertainty } \\
\hline & $-\Delta \mathrm{k}_{\mathrm{eff}}(1 \sigma)$ & $+\Delta \mathrm{k}_{\mathrm{eff}}(1 \sigma)$ & $-\Delta \mathrm{k}_{\mathrm{eff}}(1 \sigma)$ & $+\Delta \mathrm{k}_{\mathrm{eff}}(1 \sigma)$ \\
\hline Temperature & 0.00013 & -0.00013 & -- & -- \\
\hline Control Rod Positions & -0.00003 & 0.00003 & -0.00002 & 0.00003 \\
\hline Measured Value of $\mathrm{k}_{\mathrm{eff}}$ & neg & neg & neg & neg \\
\hline Kernel Diameter & \multicolumn{4}{|c|}{ Correlated Parameter (see Section 2.1.6) } \\
\hline Buffer Diameter & neg & neg & neg & neg \\
\hline IPyC Diameter & neg & 0.00001 & neg & neg \\
\hline SiC Diameter & 0.00002 & -0.00005 & neg & neg \\
\hline OPyC Diameter & neg & neg & neg & neg \\
\hline Overcoat Diameter & NA & NA & NA & NA \\
\hline Compact Inner Diameter & neg & neg & neg & neg \\
\hline Compact Outer Diameter & -0.00002 & 0.00003 & neg & neg \\
\hline Compact Height & -0.00005 & 0.00005 & neg & neg \\
\hline Compact Packing Fraction & \multicolumn{4}{|c|}{ Correlated Parameter (see Section 2.1.6) } \\
\hline Sleeve Inner Diameter & 0.00002 & -0.00003 & neg & neg \\
\hline Sleeve Outer Diameter & -0.00004 & 0.00004 & neg & neg \\
\hline Sleeve Height & -0.00001 & 0.00001 & neg & neg \\
\hline BP Diameter & 0.00015 & -0.00014 & 0.00001 & -0.00001 \\
\hline BP Stack Height & 0.00009 & -0.00007 & 0.00001 & -0.00001 \\
\hline BP Hole Diameter & 0.00001 & -0.00001 & neg & neg \\
\hline Graphite Disk Diameter & neg & neg & neg & neg \\
\hline Disk Stack Height & 0.00003 & 0.00003 & neg & neg \\
\hline CR Absorber Inner Diameter & neg & neg & neg & neg \\
\hline CR Absorber Outer Diameter & neg & neg & neg & neg \\
\hline CR Absorber Height & neg & neg & neg & neg \\
\hline CR Clad Inner Diameter & neg & neg & neg & neg \\
\hline CR Clad Outer Diameter & neg & neg & neg & neg \\
\hline CR Clad Height & neg & neg & neg & neg \\
\hline CR Spine Diameter & neg & neg & neg & neg \\
\hline Instrumentation Dimensions & -0.00139 & 0.00139 & -- & -- \\
\hline Block Flat-to-Flat Distance & -0.00004 & 0.00003 & neg & neg \\
\hline Graphite Block Height & neg & neg & neg & neg \\
\hline Dowel/Socket Dimensions & NA & NA & NA & NA \\
\hline Fuel Channel Diameter & 0.00004 & -0.00005 & neg & neg \\
\hline Reflector Channel Diameter & neg & neg & neg & neg \\
\hline Channel Pitch & -0.00004 & 0.00005 & neg & neg \\
\hline
\end{tabular}


NEA/NSC/DOC(2006)1

Gas Cooled (Thermal) Reactor - GCR

HTTR-GCR-RESR-002

CRIT-REAC-RRATE

Table 2.82 (cont'd.). Total Experimental Uncertainty (Case 1).

\begin{tabular}{|c|c|c|c|c|}
\hline \multirow{2}{*}{ Varied Parameter } & \multicolumn{2}{|c|}{ Systematic Uncertainty } & \multicolumn{2}{|c|}{ Random Uncertainty } \\
\hline & $-\Delta \mathrm{k}_{\mathrm{eff}}(1 \sigma)$ & $+\Delta \mathrm{k}_{\mathrm{eff}}(1 \sigma)$ & $-\Delta \mathrm{k}_{\mathrm{eff}}(1 \sigma)$ & $+\Delta \mathrm{k}_{\mathrm{eff}}(1 \sigma)$ \\
\hline \multirow{6}{*}{\begin{tabular}{|} 
Handling Socket Dimensions \\
Column Pitch \\
CR Channel Diameter \\
CR Channel Pitch \\
Permanent Reflector Diameter \\
Dummy Block Dimensions \\
\end{tabular}} & NA & NA & NA & NA \\
\hline & 0.00015 & -0.00018 & 0.00006 & -0.00007 \\
\hline & neg & -0.00001 & neg & neg \\
\hline & neg & neg & neg & neg \\
\hline & -0.00117 & 0.00045 & -- & -- \\
\hline & \multicolumn{4}{|c|}{ See Sections 2.1.2.7 and 2.1.3.9 } \\
\hline 3.4 wt. \% Enrichment & NA & NA & NA & NA \\
\hline 3.9 wt. \% Enrichment & 0.00005 & 0.00012 & -- & -- \\
\hline 4.3 wt. $\%$ Enrichment & -0.00054 & 0.00054 & -- & -- \\
\hline 4.8 wt. $\%$ Enrichment & -0.00023 & 0.00022 & -- & -- \\
\hline 5.2 wt. \% Enrichment & -0.00006 & 0.00008 & -- & -- \\
\hline 5.9 wt. \% Enrichment & -0.00063 & 0.00060 & -- & -- \\
\hline 6.3 wt. $\%$ Enrichment & -0.00042 & 0.00034 & -- & -- \\
\hline 6.7 wt. $\%$ Enrichment & NA & NA & NA & NA \\
\hline 7.2 wt. \% Enrichment & -0.00074 & 0.00079 & -- & -- \\
\hline 7.9 wt. \% Enrichment & -0.00038 & 0.00048 & -- & -- \\
\hline 9.4 wt. \% Enrichment & -0.00055 & 0.00062 & -- & -- \\
\hline 9.9 wt. \% Enrichment & -0.00031 & 0.00027 & -- & -- \\
\hline Oxygen to Uranium Ratio & 0.00008 & -0.00009 & -- & -- \\
\hline $\mathrm{UO}_{2}$ Density & \multicolumn{4}{|c|}{ Correlated Parameter (see Section 2.1.6) } \\
\hline $\mathrm{UO}_{2}$ Impurity & 0.00032 & -0.00056 & -- & -- \\
\hline Buffer Density & neg & neg & neg & neg \\
\hline Buffer Impurity & 0.00010 & neg & -- & -- \\
\hline IPyC Density & neg & neg & neg & neg \\
\hline IPyC Impurity & 0.00010 & 0.00010 & -- & -- \\
\hline SiC Density & NA & neg & NA & neg \\
\hline SiC Impurity & 0.00010 & neg & -- & -- \\
\hline OPyC Density & neg & neg & neg & neg \\
\hline OPyC Impurity & 0.00005 & 0.00010 & -- & -- \\
\hline Overcoat Density & -0.00001 & 0.00002 & neg & neg \\
\hline Overcoat Composition & NA & NA & NA & NA \\
\hline Overcoat Impurity & 0.00027 & -0.00040 & -- & -- \\
\hline Compact Density & neg & neg & neg & neg \\
\hline Compact Impurity & 0.00009 & -0.00043 & -- & -- \\
\hline Compact Free U Content & $\mathrm{NA}$ & neg & -- & -- \\
\hline
\end{tabular}


NEA/NSC/DOC(2006)1

Gas Cooled (Thermal) Reactor - GCR

HTTR-GCR-RESR-002

CRIT-REAC-RRATE

Table 2.82 (cont'd.). Total Experimental Uncertainty (Case 1).

\begin{tabular}{|c|c|c|c|c|}
\hline Varied Parameter & \multicolumn{2}{|c|}{ Systematic Uncertainty } & $\begin{array}{r}\text { Random } \\
-\Delta \mathrm{k}_{\mathrm{eff}}(1 \sigma) \\
\end{array}$ & $\begin{array}{l}\text { ncertainty } \\
+\Delta \mathrm{k}_{\mathrm{eff}}(1 \sigma) \\
\end{array}$ \\
\hline Sleeve Density & -0.00005 & 0.00004 & neg & neg \\
\hline Sleeve Impurity & 0.00010 & -0.00032 & -- & -- \\
\hline BP Absorber Density & 0.00012 & -0.00012 & neg & neg \\
\hline BP Absorber Content, 2.0 wt.\% & 0.00038 & -0.00034 & 0.00002 & -0.00002 \\
\hline BP Absorber Content, 2.5 wt.\% & 0.00036 & -0.00035 & 0.00003 & -0.00003 \\
\hline BP Absorber Impurity & NA & 0.00014 & -- & -- \\
\hline BP Isotopic Abundance of ${ }^{10} \mathrm{~B}$ & 0.00103 & -0.00058 & -- & -- \\
\hline Graphite Disk Density & neg & neg & neg & neg \\
\hline Graphite Disk Impurity & -0.00010 & 0.00010 & -- & -- \\
\hline CR Absorber Density & neg & -0.00001 & neg & neg \\
\hline CR Absorber Content & neg & neg & neg & neg \\
\hline CR Absorber Impurity & NA & 0.00005 & -- & -- \\
\hline CR Isotopic Abundance of ${ }^{10} \mathrm{~B}$ & 0.00010 & 0.00010 & -- & -- \\
\hline CR Clad Density & 0.00004 & 0.00002 & 0.00003 & 0.00003 \\
\hline CR Clad Composition & 0.00009 & 0.00009 & -- & -- \\
\hline CR Clad Impurity & NA & 0.00005 & -- & -- \\
\hline Instrumentation Composition & \multicolumn{4}{|c|}{ See Section 2.1.2.6 } \\
\hline IG-110 Density in Blocks & -0.00060 & 0.00061 & -0.00008 & 0.00008 \\
\hline IG-110 Impurity in Blocks & 0.00532 & -0.00659 & -- & -- \\
\hline PGX Density & -0.00010 & 0.00012 & -0.00003 & 0.00004 \\
\hline PGX Impurity & 0.00442 & -0.00585 & -- & -- \\
\hline Dummy Block Density & 0.00002 & -0.00007 & neg & -0.00003 \\
\hline Dummy Block Impurity & 0.00704 & -0.00387 & -- & -- \\
\hline Dummy Block Type & NA & -0.00004 & NA & -0.00003 \\
\hline Helium Coolant Density & neg & neg & -- & -- \\
\hline Helium Coolant Impurity & NA & neg & -- & -- \\
\hline Room Return & neg & neg & neg & neg \\
\hline TRISO Particle Placement & 0.00021 & 0.00021 & neg & neg \\
\hline Block Stack Alignment & NA & NA & NA & NA \\
\hline MCNP Random Number Seed & -- & -- & 0.00014 & 0.00014 \\
\hline Uranium Fuel Mass (Sec. 2.1.6) & -0.00073 & 0.00070 & -0.00004 & 0.00004 \\
\hline Uncertainty of Components & 0.01026 & 0.00997 & 0.00019 & 0.00019 \\
\hline Total Evaluation Uncertainty & 0.01026 & 0.00997 & & \\
\hline
\end{tabular}


NEA/NSC/DOC(2006)1

Gas Cooled (Thermal) Reactor - GCR

HTTR-GCR-RESR-002

CRIT-REAC-RRATE

Table 2.83. Total Experimental Uncertainty (Case 2).

\begin{tabular}{|c|c|c|c|c|}
\hline \multirow{2}{*}{ Varied Parameter } & \multicolumn{2}{|c|}{ Systematic Uncertainty } & \multicolumn{2}{|c|}{ Random Uncertainty } \\
\hline & $-\Delta \mathrm{k}_{\mathrm{eff}}(1 \sigma)$ & $+\Delta \mathrm{k}_{\mathrm{eff}}(1 \sigma)$ & $-\Delta \mathrm{k}_{\mathrm{eff}}(1 \sigma)$ & $+\Delta \mathrm{k}_{\mathrm{eff}}(1 \sigma)$ \\
\hline Temperature & 0.00013 & -0.00013 & -- & -- \\
\hline Control Rod Positions & -0.00011 & 0.00012 & -0.00008 & 0.00009 \\
\hline Measured Value of $\mathrm{k}_{\mathrm{eff}}$ & neg & neg & neg & neg \\
\hline Kernel Diameter & \multicolumn{4}{|c|}{ Correlated Parameter (see Section 2.1.6) } \\
\hline Buffer Diameter & neg & 0.00001 & neg & neg \\
\hline IPyC Diameter & neg & neg & neg & neg \\
\hline SiC Diameter & 0.00006 & -0.00002 & neg & neg \\
\hline OPyC Diameter & 0.00002 & 0.00003 & neg & neg \\
\hline Overcoat Diameter & NA & NA & NA & NA \\
\hline Compact Inner Diameter & neg & neg & neg & neg \\
\hline Compact Outer Diameter & -0.00002 & 0.00002 & neg & neg \\
\hline Compact Height & -0.00005 & 0.00004 & neg & neg \\
\hline Compact Packing Fraction & \multicolumn{4}{|c|}{ Correlated Parameter (see Section 2.1.6) } \\
\hline Sleeve Inner Diameter & 0.00002 & -0.00002 & neg & neg \\
\hline Sleeve Outer Diameter & -0.00004 & 0.00004 & neg & neg \\
\hline Sleeve Height & -0.00001 & 0.00001 & neg & neg \\
\hline BP Diameter & 0.00016 & -0.00015 & 0.00001 & -0.00001 \\
\hline BP Stack Height & 0.00008 & -0.00009 & neg & neg \\
\hline BP Hole Diameter & 0.00002 & -0.00001 & neg & neg \\
\hline Graphite Disk Diameter & neg & neg & neg & neg \\
\hline Disk Stack Height & 0.00002 & 0.00002 & neg & neg \\
\hline CR Absorber Inner Diameter & neg & neg & neg & neg \\
\hline CR Absorber Outer Diameter & neg & neg & neg & neg \\
\hline CR Absorber Height & 0.00001 & -0.00001 & 0.00001 & -0.00001 \\
\hline CR Clad Inner Diameter & neg & neg & neg & neg \\
\hline CR Clad Outer Diameter & neg & neg & neg & neg \\
\hline CR Clad Height & -0.00003 & -0.00003 & -0.00001 & -0.00001 \\
\hline CR Spine Diameter & neg & neg & neg & neg \\
\hline Instrumentation Dimensions & -0.00116 & 0.00116 & -- & -- \\
\hline Block Flat-to-Flat Distance & -0.00003 & 0.00003 & neg & neg \\
\hline Graphite Block Height & neg & 0.00002 & neg & neg \\
\hline Dowel/Socket Dimensions & NA & NA & NA & NA \\
\hline Fuel Channel Diameter & 0.00005 & -0.00005 & neg & neg \\
\hline Reflector Channel Diameter & neg & neg & neg & neg \\
\hline Channel Pitch & -0.00004 & 0.00004 & neg & neg \\
\hline
\end{tabular}


NEA/NSC/DOC(2006)1

Gas Cooled (Thermal) Reactor - GCR

HTTR-GCR-RESR-002

CRIT-REAC-RRATE

Table 2.83 (cont'd.). Total Experimental Uncertainty (Case 2).

\begin{tabular}{|c|c|c|c|c|}
\hline \multirow{2}{*}{ Varied Parameter } & \multicolumn{2}{|c|}{ Systematic Uncertainty } & \multicolumn{2}{|c|}{ Random Uncertainty } \\
\hline & $-\Delta \mathrm{k}_{\mathrm{eff}}(1 \sigma)$ & $+\Delta \mathrm{k}_{\mathrm{eff}}(1 \sigma)$ & $-\Delta \mathrm{k}_{\mathrm{eff}}(1 \sigma)$ & $+\Delta \mathrm{k}_{\mathrm{eff}}(1 \sigma)$ \\
\hline Handling Socket Dimensions & NA & NA & NA & NA \\
\hline Column Pitch & 0.00016 & -0.00017 & 0.00006 & -0.00006 \\
\hline CR Channel Diameter & neg & neg & neg & neg \\
\hline CR Channel Pitch & neg & neg & neg & neg \\
\hline Permanent Reflector Diameter & -0.00083 & 0.00043 & -- & -- \\
\hline Dummy Block Dimensions & \multicolumn{4}{|c|}{ See Sections 2.1.2.7 and 2.1.3.9 } \\
\hline 3.4 wt. \% Enrichment & NA & NA & NA & NA \\
\hline 3.9 wt. \% Enrichment & -0.00030 & 0.00023 & -- & -- \\
\hline 4.3 wt. $\%$ Enrichment & -0.00081 & 0.00073 & -- & -- \\
\hline 4.8 wt. $\%$ Enrichment & -0.00041 & 0.00037 & -- & -- \\
\hline 5.2 wt. \% Enrichment & -0.00033 & 0.00017 & -- & -- \\
\hline 5.9 wt. \% Enrichment & -0.00076 & 0.00076 & -- & -- \\
\hline 6.3 wt. $\%$ Enrichment & -0.00067 & 0.00047 & -- & -- \\
\hline 6.7 wt. \% Enrichment & NA & NA & NA & NA \\
\hline 7.2 wt. \% Enrichment & -0.00071 & 0.00060 & -- & -- \\
\hline 7.9 wt. \% Enrichment & -0.00038 & 0.00031 & -- & -- \\
\hline 9.4 wt. \% Enrichment & -0.00026 & 0.00016 & -- & -- \\
\hline 9.9 wt. \% Enrichment & -0.00015 & 0.00005 & -- & -- \\
\hline Oxygen to Uranium Ratio & 0.00014 & -0.00007 & -- & -- \\
\hline $\mathrm{UO}_{2}$ Density & \multicolumn{4}{|c|}{ Correlated Parameter (see Section 2.1.6) } \\
\hline $\mathrm{UO}_{2}$ Impurity & 0.00047 & -0.00060 & -- & -- \\
\hline Buffer Density & neg & 0.00001 & neg & neg \\
\hline Buffer Impurity & 0.00010 & neg & -- & -- \\
\hline IPyC Density & neg & 0.00001 & neg & neg \\
\hline IPyC Impurity & 0.00009 & 0.00010 & -- & -- \\
\hline SiC Density & NA & neg & NA & neg \\
\hline SiC Impurity & 0.00010 & 0.00009 & -- & -- \\
\hline OPyC Density & neg & 0.00001 & neg & neg \\
\hline OPyC Impurity & 0.00024 & -0.00015 & -- & -- \\
\hline Overcoat Density & neg & 0.00002 & neg & neg \\
\hline Overcoat Composition & NA & NA & NA & NA \\
\hline Overcoat Impurity & 0.00030 & -0.00059 & -- & -- \\
\hline Compact Density & neg & neg & neg & neg \\
\hline Compact Impurity & 0.00013 & -0.00042 & -- & -- \\
\hline Compact Free U Content & NA & neg & -- & -- \\
\hline
\end{tabular}


NEA/NSC/DOC(2006)1

Gas Cooled (Thermal) Reactor - GCR

HTTR-GCR-RESR-002

CRIT-REAC-RRATE

Table 2.83 (cont'd.). Total Experimental Uncertainty (Case 2).

\begin{tabular}{|c|c|c|c|c|}
\hline Varied Parameter & $\begin{array}{l}\text { Systematic } \\
-\Delta \mathrm{k}_{\mathrm{eff}}(1 \sigma) \\
\end{array}$ & $\begin{array}{l}\text { Jncertainty } \\
+\Delta \mathrm{k}_{\mathrm{eff}}(1 \sigma) \\
\end{array}$ & $\begin{array}{r}\text { Random } \\
-\Delta \mathrm{k}_{\mathrm{eff}}(1 \sigma) \\
\end{array}$ & $\begin{array}{l}\text { ncertainty } \\
+\Delta \mathrm{k}_{\mathrm{eff}}(1 \sigma)\end{array}$ \\
\hline Sleeve Density & -0.00003 & 0.00003 & neg & neg \\
\hline Sleeve Impurity & 0.00018 & -0.00021 & -- & -- \\
\hline BP Absorber Density & 0.00015 & -0.00007 & neg & neg \\
\hline BP Absorber Content, 2.0 wt. \% & 0.00044 & -0.00034 & 0.00003 & -0.00002 \\
\hline BP Absorber Content, 2.5 wt. \% & 0.00041 & -0.00036 & 0.00003 & -0.00003 \\
\hline BP Absorber Impurity & NA & 0.00023 & -- & -- \\
\hline BP Isotopic Abundance of ${ }^{10} \mathrm{~B}$ & 0.00131 & -0.00042 & -- & -- \\
\hline Graphite Disk Density & neg & 0.00001 & neg & neg \\
\hline Graphite Disk Impurity & 0.00009 & 0.00010 & -- & -- \\
\hline CR Absorber Density & neg & neg & neg & neg \\
\hline CR Absorber Content & 0.00003 & -0.00001 & neg & neg \\
\hline CR Absorber Impurity & NA & -0.00005 & -- & -- \\
\hline CR Isotopic Abundance of ${ }^{10} \mathrm{~B}$ & 0.00010 & neg & -- & -- \\
\hline CR Clad Density & 0.00002 & neg & neg & neg \\
\hline CR Clad Composition & 0.00010 & 0.00010 & -- & -- \\
\hline CR Clad Impurity & NA & 0.00005 & -- & -- \\
\hline Instrumentation Composition & \multicolumn{4}{|c|}{ See Section 2.1.2.6 } \\
\hline IG-110 Density in Blocks & -0.00061 & 0.00066 & -0.00008 & 0.00009 \\
\hline IG-110 Impurity in Blocks & 0.00486 & -0.00617 & -- & -- \\
\hline PGX Density & -0.00010 & 0.00014 & -0.00003 & 0.00004 \\
\hline PGX Impurity & 0.00379 & -0.00499 & -- & -- \\
\hline Dummy Block Density & 0.00008 & -0.00008 & 0.00003 & -0.00004 \\
\hline Dummy Block Impurity & 0.00738 & -0.00389 & -- & -- \\
\hline Dummy Block Type & NA & -0.00037 & NA & -0.00016 \\
\hline Helium Coolant Density & neg & 0.00001 & -- & -- \\
\hline Helium Coolant Impurity & NA & neg & -- & -- \\
\hline Room Return & neg & neg & neg & neg \\
\hline TRISO Particle Placement & 0.00033 & 0.00033 & neg & neg \\
\hline Block Stack Alignment & NA & NA & NA & NA \\
\hline MCNP Random Number Seed & -- & -- & 0.00008 & 0.00008 \\
\hline Uranium Fuel Mass (Sec. 2.1.6) & -0.00070 & 0.00074 & -0.00004 & 0.00004 \\
\hline Uncertainty of Components & 0.01005 & 0.00919 & 0.00017 & 0.00024 \\
\hline Total Evaluation Uncertainty & 0.01004 & 0.00919 & & \\
\hline
\end{tabular}


NEA/NSC/DOC(2006)1

Gas Cooled (Thermal) Reactor - GCR

HTTR-GCR-RESR-002

CRIT-REAC-RRATE

Table 2.84. Total Experimental Uncertainty (Case 3).

\begin{tabular}{|c|c|c|c|c|}
\hline \multirow{2}{*}{ Varied Parameter } & \multicolumn{2}{|c|}{ Systematic Uncertainty } & \multicolumn{2}{|c|}{ Random Uncertainty } \\
\hline & $-\Delta \mathrm{k}_{\mathrm{eff}}(1 \sigma)$ & $+\Delta \mathrm{k}_{\mathrm{eff}}(1 \sigma)$ & $-\Delta \mathrm{k}_{\mathrm{eff}}(1 \sigma)$ & $+\Delta \mathrm{k}_{\mathrm{eff}}(1 \sigma)$ \\
\hline Temperature & 0.00013 & -0.00013 & -- & -- \\
\hline Control Rod Positions & -0.00015 & 0.00013 & -0.00011 & 0.00010 \\
\hline Measured Value of $\mathrm{k}_{\mathrm{eff}}$ & neg & neg & neg & neg \\
\hline Kernel Diameter & \multicolumn{4}{|c|}{ Correlated Parameter (see Section 2.1.6) } \\
\hline Buffer Diameter & -0.00002 & 0.00001 & neg & neg \\
\hline IPyC Diameter & -0.00001 & neg & neg & neg \\
\hline SiC Diameter & 0.00005 & -0.00005 & neg & neg \\
\hline OPyC Diameter & -0.00002 & neg & neg & neg \\
\hline Overcoat Diameter & NA & NA & NA & NA \\
\hline Compact Inner Diameter & neg & neg & neg & neg \\
\hline Compact Outer Diameter & -0.00004 & 0.00003 & neg & neg \\
\hline Compact Height & -0.00005 & 0.00004 & neg & neg \\
\hline Compact Packing Fraction & \multicolumn{4}{|c|}{ Correlated Parameter (see Section 2.1.6) } \\
\hline Sleeve Inner Diameter & 0.00002 & -0.00003 & neg & neg \\
\hline Sleeve Outer Diameter & -0.00004 & 0.00005 & neg & neg \\
\hline Sleeve Height & -0.00001 & 0.00001 & neg & neg \\
\hline BP Diameter & 0.00017 & -0.00017 & 0.00001 & -0.00001 \\
\hline BP Stack Height & 0.00006 & -0.00011 & 0.00001 & -0.00002 \\
\hline BP Hole Diameter & 0.00001 & -0.00002 & neg & neg \\
\hline Graphite Disk Diameter & neg & neg & neg & neg \\
\hline Disk Stack Height & neg & neg & neg & neg \\
\hline CR Absorber Inner Diameter & neg & neg & neg & neg \\
\hline CR Absorber Outer Diameter & neg & neg & neg & neg \\
\hline CR Absorber Height & 0.00001 & -0.00001 & neg & neg \\
\hline CR Clad Inner Diameter & neg & neg & neg & neg \\
\hline CR Clad Outer Diameter & -0.00001 & 0.00001 & neg & neg \\
\hline CR Clad Height & -0.00004 & -0.00003 & -0.00001 & -0.00001 \\
\hline CR Spine Diameter & neg & neg & neg & neg \\
\hline Instrumentation Dimensions & -0.00100 & 0.00100 & -- & -- \\
\hline Block Flat-to-Flat Distance & -0.00005 & 0.00004 & -0.00001 & neg \\
\hline Graphite Block Height & neg & neg & neg & neg \\
\hline Dowel/Socket Dimensions & NA & NA & NA & NA \\
\hline Fuel Channel Diameter & 0.00005 & -0.00006 & neg & neg \\
\hline Reflector Channel Diameter & neg & neg & neg & neg \\
\hline Channel Pitch & -0.00004 & 0.00003 & neg & neg \\
\hline
\end{tabular}


NEA/NSC/DOC(2006)1

Gas Cooled (Thermal) Reactor - GCR

HTTR-GCR-RESR-002

CRIT-REAC-RRATE

Table 2.84 (cont'd.). Total Experimental Uncertainty (Case 3).

\begin{tabular}{|c|c|c|c|c|}
\hline \multirow{2}{*}{ Varied Parameter } & \multicolumn{2}{|c|}{ Systematic Uncertainty } & \multicolumn{2}{|c|}{ Random Uncertainty } \\
\hline & $-\Delta \mathrm{k}_{\mathrm{eff}}(1 \sigma)$ & $+\Delta \mathrm{k}_{\mathrm{eff}}(1 \sigma)$ & $-\Delta \mathrm{k}_{\mathrm{eff}}(1 \sigma)$ & $+\Delta \mathrm{k}_{\mathrm{eff}}(1 \sigma)$ \\
\hline Handling Socket Dimensions & NA & NA & NA & NA \\
\hline Column Pitch & 0.00015 & -0.00018 & 0.00006 & -0.00007 \\
\hline CR Channel Diameter & 0.00001 & -0.00001 & neg & neg \\
\hline CR Channel Pitch & neg & neg & neg & neg \\
\hline Permanent Reflector Diameter & -0.00084 & 0.00034 & -- & -- \\
\hline Dummy Block Dimensions & \multicolumn{4}{|c|}{ See Sections 2.1.2.7 and 2.1.3.9 } \\
\hline 3.4 wt. \% Enrichment & NA & NA & NA & NA \\
\hline 3.9 wt. \% Enrichment & -0.00064 & 0.00077 & -- & -- \\
\hline 4.3 wt. $\%$ Enrichment & -0.00092 & 0.00098 & -- & -- \\
\hline 4.8 wt. $\%$ Enrichment & -0.00043 & 0.00052 & -- & -- \\
\hline 5.2 wt. \% Enrichment & -0.00046 & 0.00049 & -- & -- \\
\hline 5.9 wt. \% Enrichment & -0.00058 & 0.00071 & -- & -- \\
\hline 6.3 wt. $\%$ Enrichment & -0.00045 & 0.00052 & -- & -- \\
\hline 6.7 wt. $\%$ Enrichment & NA & NA & NA & NA \\
\hline 7.2 wt. \% Enrichment & -0.00031 & 0.00036 & -- & -- \\
\hline 7.9 wt. \% Enrichment & -0.00011 & 0.00017 & -- & -- \\
\hline 9.4 wt. \% Enrichment & 0.00005 & 0.00013 & -- & -- \\
\hline 9.9 wt.\% Enrichment & 0.00005 & 0.00005 & -- & -- \\
\hline Oxygen to Uranium Ratio & 0.00007 & -0.00017 & -- & -- \\
\hline $\mathrm{UO}_{2}$ Density & \multicolumn{4}{|c|}{ Correlated Parameter (see Section 2.1.6) } \\
\hline $\mathrm{UO}_{2}$ Impurity & 0.00024 & -0.00068 & -- & -- \\
\hline Buffer Density & -0.00001 & neg & neg & neg \\
\hline Buffer Impurity & 0.00009 & -0.00012 & -- & -- \\
\hline IPyC Density & neg & neg & neg & neg \\
\hline IPyC Impurity & 0.00011 & -0.00016 & -- & -- \\
\hline SiC Density & NA & neg & NA & neg \\
\hline SiC Impurity & 0.00010 & 0.00010 & -- & -- \\
\hline OPyC Density & neg & -0.00001 & neg & neg \\
\hline OPyC Impurity & 0.00005 & 0.00010 & -- & -- \\
\hline Overcoat Density & -0.00002 & neg & neg & neg \\
\hline Overcoat Composition & NA & NA & NA & NA \\
\hline Overcoat Impurity & 0.00044 & -0.00070 & -- & -- \\
\hline Compact Density & -0.00001 & neg & neg & neg \\
\hline Compact Impurity & 0.00010 & -0.00066 & -- & -- \\
\hline Compact Free U Content & NA & neg & -- & -- \\
\hline
\end{tabular}


NEA/NSC/DOC(2006)1

Gas Cooled (Thermal) Reactor - GCR

HTTR-GCR-RESR-002

CRIT-REAC-RRATE

Table 2.84 (cont'd.). Total Experimental Uncertainty (Case 3).

\begin{tabular}{|c|c|c|c|c|}
\hline Varied Parameter & $\begin{array}{l}\text { Systematic } \\
-\Delta \mathrm{k}_{\mathrm{eff}}(1 \sigma) \\
\end{array}$ & $\begin{array}{l}\text { Uncertainty } \\
+\Delta \mathrm{k}_{\mathrm{eff}}(1 \sigma) \\
\end{array}$ & $\begin{array}{r}\text { Random } \\
-\Delta \mathrm{k}_{\mathrm{eff}}(1 \sigma) \\
\end{array}$ & $\begin{array}{l}\text { ncertainty } \\
+\Delta \mathrm{k}_{\mathrm{eff}}(1 \sigma) \\
\end{array}$ \\
\hline Sleeve Density & -0.00008 & 0.00004 & neg & neg \\
\hline Sleeve Impurity & 0.00010 & -0.00048 & -- & -- \\
\hline BP Absorber Density & 0.00010 & -0.00012 & neg & neg \\
\hline BP Absorber Content, 2.0 wt. \% & 0.00057 & -0.00049 & 0.00003 & -0.00003 \\
\hline BP Absorber Content, 2.5 wt. \% & 0.00036 & -0.00035 & 0.00003 & -0.00003 \\
\hline BP Absorber Impurity & NA & 0.00021 & -- & -- \\
\hline BP Isotopic Abundance of ${ }^{10} \mathrm{~B}$ & 0.00126 & -0.00062 & -- & -- \\
\hline Graphite Disk Density & neg & neg & neg & neg \\
\hline Graphite Disk Impurity & 0.00010 & -0.00010 & -- & -- \\
\hline CR Absorber Density & neg & neg & neg & neg \\
\hline CR Absorber Content & 0.00002 & -0.00002 & neg & neg \\
\hline CR Absorber Impurity & NA & 0.00005 & -- & -- \\
\hline CR Isotopic Abundance of ${ }^{10} \mathrm{~B}$ & 0.00013 & 0.00009 & -- & -- \\
\hline CR Clad Density & neg & -0.00003 & neg & -0.00001 \\
\hline CR Clad Composition & 0.00010 & -0.00012 & -- & -- \\
\hline CR Clad Impurity & NA & 0.00005 & -- & -- \\
\hline Instrumentation Composition & \multicolumn{4}{|c|}{ See Section 2.1.2.6 } \\
\hline IG-110 Density in Blocks & -0.00073 & 0.00063 & -0.00010 & 0.00008 \\
\hline IG-110 Impurity in Blocks & 0.00462 & -0.00601 & -- & -- \\
\hline PGX Density & -0.00009 & 0.00004 & -0.00003 & 0.00001 \\
\hline PGX Impurity & 0.00314 & -0.00434 & -- & -- \\
\hline Dummy Block Density & 0.00002 & -0.00007 & neg & -0.00004 \\
\hline Dummy Block Impurity & 0.00460 & -0.00264 & -- & -- \\
\hline Dummy Block Type & NA & -0.00014 & NA & -0.00008 \\
\hline Helium Coolant Density & neg & neg & -- & -- \\
\hline Helium Coolant Impurity & NA & neg & -- & -- \\
\hline Room Return & neg & neg & neg & Neg \\
\hline TRISO Particle Placement & 0.00051 & 0.00051 & neg & neg \\
\hline Block Stack Alignment & NA & NA & NA & NA \\
\hline MCNP Random Number Seed & -- & -- & 0.00007 & 0.00007 \\
\hline Uranium Fuel Mass (Sec. 2.1.6) & -0.00077 & 0.00071 & -0.00004 & 0.00003 \\
\hline Uncertainty of Components & 0.00776 & 0.00836 & 0.00018 & 0.00019 \\
\hline Total Evaluation Uncertainty & 0.00777 & 0.00836 & & \\
\hline
\end{tabular}


NEA/NSC/DOC(2006)1

Gas Cooled (Thermal) Reactor - GCR

HTTR-GCR-RESR-002

CRIT-REAC-RRATE

Table 2.85. Total Experimental Uncertainty (Case 4).

\begin{tabular}{|c|c|c|c|c|}
\hline \multirow{2}{*}{ Varied Parameter } & \multicolumn{2}{|c|}{ Systematic Uncertainty } & \multicolumn{2}{|c|}{ Random Uncertainty } \\
\hline & $-\Delta \mathrm{k}_{\mathrm{eff}}(1 \sigma)$ & $+\Delta \mathrm{k}_{\mathrm{eff}}(1 \sigma)$ & $-\Delta \mathrm{k}_{\mathrm{eff}}(1 \sigma)$ & $+\Delta \mathrm{k}_{\mathrm{eff}}(1 \sigma)$ \\
\hline Temperature & 0.00013 & -0.00013 & -- & -- \\
\hline Control Rod Positions & -0.00009 & 0.00006 & -0.00007 & 0.00005 \\
\hline Measured Value of $\mathrm{k}_{\mathrm{eff}}$ & neg & neg & neg & neg \\
\hline Kernel Diameter & \multicolumn{4}{|c|}{ Correlated Parameter (see Section 2.1.6) } \\
\hline Buffer Diameter & neg & -0.00004 & neg & neg \\
\hline IPyC Diameter & neg & neg & neg & neg \\
\hline SiC Diameter & 0.00002 & -0.00007 & neg & neg \\
\hline OPyC Diameter & 0.00001 & -0.00003 & neg & neg \\
\hline Overcoat Diameter & NA & NA & NA & NA \\
\hline Compact Inner Diameter & neg & -0.00002 & neg & neg \\
\hline Compact Outer Diameter & -0.00004 & 0.00003 & neg & neg \\
\hline Compact Height & -0.00006 & 0.00004 & neg & neg \\
\hline Compact Packing Fraction & \multicolumn{4}{|c|}{ Correlated Parameter (see Section 2.1.6) } \\
\hline Sleeve Inner Diameter & 0.00003 & -0.00003 & neg & neg \\
\hline Sleeve Outer Diameter & -0.00005 & 0.00005 & neg & neg \\
\hline Sleeve Height & -0.00002 & 0.00001 & neg & neg \\
\hline BP Diameter & 0.00017 & -0.00017 & 0.00001 & -0.00001 \\
\hline BP Stack Height & 0.00007 & -0.00013 & 0.00001 & -0.00002 \\
\hline BP Hole Diameter & 0.00001 & -0.00002 & neg & neg \\
\hline Graphite Disk Diameter & neg & neg & neg & neg \\
\hline Disk Stack Height & neg & 0.00002 & neg & neg \\
\hline CR Absorber Inner Diameter & neg & neg & neg & neg \\
\hline CR Absorber Outer Diameter & neg & neg & neg & neg \\
\hline CR Absorber Height & 0.00001 & -0.00001 & neg & neg \\
\hline CR Clad Inner Diameter & neg & neg & neg & neg \\
\hline CR Clad Outer Diameter & -0.00002 & 0.00002 & neg & neg \\
\hline CR Clad Height & -0.00002 & -0.00002 & neg & neg \\
\hline CR Spine Diameter & neg & neg & neg & neg \\
\hline Instrumentation Dimensions & -0.00091 & 0.00091 & -- & -- \\
\hline Block Flat-to-Flat Distance & -0.00006 & 0.00004 & -0.00001 & 0.00001 \\
\hline Graphite Block Height & neg & neg & neg & neg \\
\hline Dowel/Socket Dimensions & NA & NA & NA & NA \\
\hline Fuel Channel Diameter & 0.00006 & -0.00007 & neg & neg \\
\hline Reflector Channel Diameter & neg & neg & neg & neg \\
\hline Channel Pitch & -0.00004 & 0.00003 & neg & neg \\
\hline
\end{tabular}


NEA/NSC/DOC(2006)1

Gas Cooled (Thermal) Reactor - GCR

HTTR-GCR-RESR-002

CRIT-REAC-RRATE

Table 2.85 (cont'd.). Total Experimental Uncertainty (Case 4).

\begin{tabular}{|c|c|c|c|c|}
\hline \multirow{2}{*}{ Varied Parameter } & \multicolumn{2}{|c|}{ Systematic Uncertainty } & \multicolumn{2}{|c|}{ Random Uncertainty } \\
\hline & $-\Delta \mathrm{k}_{\mathrm{eff}}(1 \sigma)$ & $+\Delta \mathrm{k}_{\mathrm{eff}}(1 \sigma)$ & $-\Delta \mathrm{k}_{\mathrm{eff}}(1 \sigma)$ & $+\Delta \mathrm{k}_{\mathrm{eff}}(1 \sigma)$ \\
\hline Handling Socket Dimensions & NA & NA & NA & NA \\
\hline Column Pitch & 0.00013 & -0.00019 & 0.00005 & -0.00007 \\
\hline CR Channel Diameter & 0.00001 & -0.00001 & neg & neg \\
\hline CR Channel Pitch & neg & neg & neg & neg \\
\hline Permanent Reflector Diameter & -0.00054 & 0.00014 & -- & -- \\
\hline Dummy Block Dimensions & \multicolumn{4}{|c|}{ See Sections 2.1.2.7 and 2.1.3.9 } \\
\hline 3.4 wt. \% Enrichment & NA & NA & NA & NA \\
\hline 3.9 wt. \% Enrichment & -0.00053 & 0.00059 & -- & -- \\
\hline 4.3 wt. $\%$ Enrichment & -0.00064 & 0.00079 & -- & -- \\
\hline 4.8 wt. $\%$ Enrichment & -0.00030 & 0.00036 & -- & -- \\
\hline 5.2 wt. \% Enrichment & -0.00045 & 0.00051 & -- & -- \\
\hline 5.9 wt. \% Enrichment & -0.00048 & 0.00041 & -- & -- \\
\hline 6.3 wt. $\%$ Enrichment & -0.00063 & 0.00066 & -- & -- \\
\hline 6.7 wt. $\%$ Enrichment & NA & NA & NA & NA \\
\hline 7.2 wt. \% Enrichment & -0.00034 & 0.00033 & -- & -- \\
\hline 7.9 wt. \% Enrichment & -0.00037 & 0.00044 & -- & -- \\
\hline 9.4 wt. \% Enrichment & -0.00014 & 0.00018 & -- & -- \\
\hline 9.9 wt. \% Enrichment & 0.00006 & 0.00011 & -- & -- \\
\hline Oxygen to Uranium Ratio & 0.00011 & -0.00012 & -- & -- \\
\hline $\mathrm{UO}_{2}$ Density & \multicolumn{4}{|c|}{ Correlated Parameter (see Section 2.1.6) } \\
\hline $\mathrm{UO}_{2}$ Impurity & 0.00026 & -0.00063 & -- & -- \\
\hline Buffer Density & neg & neg & neg & neg \\
\hline Buffer Impurity & 0.00010 & -0.00010 & -- & -- \\
\hline IPyC Density & neg & neg & neg & neg \\
\hline IPyC Impurity & 0.00010 & 0.00010 & -- & -- \\
\hline SiC Density & NA & neg & NA & neg \\
\hline SiC Impurity & neg & -0.00011 & -- & -- \\
\hline OPyC Density & neg & neg & neg & neg \\
\hline OPyC Impurity & -0.00012 & 0.00010 & -- & -- \\
\hline Overcoat Density & -0.00003 & 0.00002 & neg & neg \\
\hline Overcoat Composition & NA & NA & NA & NA \\
\hline Overcoat Impurity & 0.00025 & -0.00076 & -- & -- \\
\hline Compact Density & -0.00001 & 0.00001 & neg & neg \\
\hline Compact Impurity & 0.00010 & -0.00060 & -- & -- \\
\hline Compact Free U Content & NA & neg & -- & -- \\
\hline
\end{tabular}


NEA/NSC/DOC(2006)1

Gas Cooled (Thermal) Reactor - GCR

HTTR-GCR-RESR-002

CRIT-REAC-RRATE

Table 2.85 (cont'd.). Total Experimental Uncertainty (Case 4).

\begin{tabular}{|c|c|c|c|c|}
\hline Varied Parameter & $\begin{array}{l}\text { Systematic } \\
-\Delta \mathrm{k}_{\mathrm{eff}}(1 \sigma) \\
\end{array}$ & $\begin{array}{l}\text { Uncertainty } \\
+\Delta \mathrm{k}_{\mathrm{eff}}(1 \sigma) \\
\end{array}$ & $\begin{array}{r}\text { Random } \\
-\Delta \mathrm{k}_{\mathrm{eff}}(1 \sigma) \\
\end{array}$ & $\begin{array}{l}\text { ncertainty } \\
+\Delta \mathrm{k}_{\mathrm{eff}}(1 \sigma) \\
\end{array}$ \\
\hline Sleeve Density & -0.00012 & 0.00006 & neg & neg \\
\hline Sleeve Impurity & 0.00009 & -0.00033 & -- & -- \\
\hline BP Absorber Density & 0.00009 & -0.00012 & neg & neg \\
\hline BP Absorber Content, 2.0 wt. \% & 0.00055 & -0.00050 & 0.00003 & -0.00003 \\
\hline BP Absorber Content, 2.5 wt. \% & 0.00041 & -0.00036 & 0.00003 & -0.00003 \\
\hline BP Absorber Impurity & NA & 0.00018 & -- & -- \\
\hline BP Isotopic Abundance of ${ }^{10} \mathrm{~B}$ & 0.00127 & -0.00080 & -- & -- \\
\hline Graphite Disk Density & -0.00001 & neg & neg & neg \\
\hline Graphite Disk Impurity & -0.00010 & 0.00010 & -- & -- \\
\hline CR Absorber Density & neg & -0.00001 & neg & neg \\
\hline CR Absorber Content & 0.00003 & -0.00003 & neg & neg \\
\hline CR Absorber Impurity & NA & 0.00005 & -- & -- \\
\hline CR Isotopic Abundance of ${ }^{10} \mathrm{~B}$ & 0.00010 & -0.00010 & -- & -- \\
\hline CR Clad Density & -0.00005 & neg & -0.00002 & neg \\
\hline CR Clad Composition & 0.00010 & 0.00010 & -- & -- \\
\hline CR Clad Impurity & NA & neg & -- & -- \\
\hline Instrumentation Composition & \multicolumn{4}{|c|}{ See Section 2.1.2.6 } \\
\hline IG-110 Density in Blocks & -0.00083 & 0.00080 & -0.00011 & 0.00011 \\
\hline IG-110 Impurity in Blocks & 0.00420 & -0.00543 & -- & -- \\
\hline PGX Density & -0.00007 & 0.00005 & -0.00002 & 0.00001 \\
\hline PGX Impurity & 0.00171 & -0.00247 & -- & -- \\
\hline Dummy Block Density & 0.00002 & -0.00007 & 0.00001 & -0.00004 \\
\hline Dummy Block Impurity & 0.00599 & -0.00335 & -- & -- \\
\hline Dummy Block Type & NA & -0.00064 & NA & -0.00035 \\
\hline Helium Coolant Density & -0.00001 & neg & -- & -- \\
\hline Helium Coolant Impurity & NA & neg & -- & -- \\
\hline Room Return & neg & neg & neg & neg \\
\hline TRISO Particle Placement & 0.00053 & 0.00053 & neg & neg \\
\hline Block Stack Alignment & NA & NA & NA & NA \\
\hline MCNP Random Number Seed & -- & -- & 0.00012 & 0.00012 \\
\hline Uranium Fuel Mass (Sec. 2.1.6) & -0.00075 & 0.00072 & -0.00004 & 0.00004 \\
\hline Uncertainty of Components & 0.00796 & 0.00739 & 0.00020 & 0.00040 \\
\hline Total Evaluation Uncertainty & 0.00796 & 0.00740 & & \\
\hline
\end{tabular}


NEA/NSC/DOC(2006)1

Gas Cooled (Thermal) Reactor - GCR

HTTR-GCR-RESR-002

CRIT-REAC-RRATE

Table 2.86. Total Experimental Uncertainty (Case 5).

\begin{tabular}{|c|c|c|c|c|}
\hline \multirow{2}{*}{ Varied Parameter } & \multicolumn{2}{|c|}{ Systematic Uncertainty } & \multicolumn{2}{|c|}{ Random Uncertainty } \\
\hline & $-\Delta \mathrm{k}_{\mathrm{eff}}(1 \sigma)$ & $+\Delta \mathrm{k}_{\mathrm{eff}}(1 \sigma)$ & $-\Delta \mathrm{k}_{\mathrm{eff}}(1 \sigma)$ & $+\Delta \mathrm{k}_{\mathrm{eff}}(1 \sigma)$ \\
\hline Temperature & 0.00013 & -0.00013 & -- & -- \\
\hline Control Rod Positions & -0.00014 & 0.00016 & -0.00011 & 0.00012 \\
\hline Measured Value of $\mathrm{k}_{\mathrm{eff}}$ & neg & neg & neg & neg \\
\hline Kernel Diameter & \multicolumn{4}{|c|}{ Correlated Parameter (see Section 2.1.6) } \\
\hline Buffer Diameter & 0.00002 & -0.00002 & neg & neg \\
\hline IPyC Diameter & neg & neg & neg & neg \\
\hline SiC Diameter & 0.00006 & -0.00006 & neg & neg \\
\hline OPyC Diameter & neg & neg & neg & neg \\
\hline Overcoat Diameter & NA & NA & NA & NA \\
\hline Compact Inner Diameter & 0.00001 & -0.00001 & neg & neg \\
\hline Compact Outer Diameter & -0.00003 & 0.00003 & neg & neg \\
\hline Compact Height & -0.00006 & 0.00004 & neg & neg \\
\hline Compact Packing Fraction & \multicolumn{4}{|c|}{ Correlated Parameter (see Section 2.1.6) } \\
\hline Sleeve Inner Diameter & 0.00003 & -0.00004 & neg & neg \\
\hline Sleeve Outer Diameter & -0.00005 & 0.00005 & neg & neg \\
\hline Sleeve Height & -0.00002 & 0.00001 & neg & neg \\
\hline BP Diameter & 0.00019 & -0.00018 & 0.00001 & -0.00001 \\
\hline BP Stack Height & 0.00008 & -0.00009 & 0.00001 & -0.00001 \\
\hline BP Hole Diameter & 0.00002 & -0.00001 & neg & neg \\
\hline Graphite Disk Diameter & neg & neg & neg & neg \\
\hline Disk Stack Height & 0.00002 & 0.00003 & neg & neg \\
\hline CR Absorber Inner Diameter & neg & neg & neg & neg \\
\hline CR Absorber Outer Diameter & neg & neg & neg & neg \\
\hline CR Absorber Height & 0.00001 & -0.00002 & 0.00001 & -0.00001 \\
\hline CR Clad Inner Diameter & neg & neg & neg & neg \\
\hline CR Clad Outer Diameter & -0.00001 & 0.00001 & neg & neg \\
\hline CR Clad Height & -0.00003 & -0.00004 & -0.00001 & -0.00001 \\
\hline CR Spine Diameter & neg & neg & neg & neg \\
\hline Instrumentation Dimensions & -0.00084 & 0.00084 & -- & -- \\
\hline Block Flat-to-Flat Distance & -0.00006 & 0.00004 & -0.00001 & 0.00001 \\
\hline Graphite Block Height & neg & neg & neg & neg \\
\hline Dowel/Socket Dimensions & NA & NA & NA & NA \\
\hline Fuel Channel Diameter & 0.00006 & -0.00006 & neg & neg \\
\hline Reflector Channel Diameter & neg & neg & neg & neg \\
\hline Channel Pitch & -0.00003 & 0.00003 & neg & neg \\
\hline
\end{tabular}


NEA/NSC/DOC(2006)1

Gas Cooled (Thermal) Reactor - GCR

HTTR-GCR-RESR-002

CRIT-REAC-RRATE

Table 2.86 (cont'd.). Total Experimental Uncertainty (Case 5).

\begin{tabular}{|c|c|c|c|c|}
\hline \multirow{2}{*}{ Varied Parameter } & \multicolumn{2}{|c|}{ Systematic Uncertainty } & \multicolumn{2}{|c|}{ Random Uncertainty } \\
\hline & $-\Delta \mathrm{k}_{\mathrm{eff}}(1 \sigma)$ & $+\Delta \mathrm{k}_{\mathrm{eff}}(1 \sigma)$ & $-\Delta \mathrm{k}_{\mathrm{eff}}(1 \sigma)$ & $+\Delta \mathrm{k}_{\mathrm{eff}}(1 \sigma)$ \\
\hline Handling Socket Dimensions & NA & NA & NA & NA \\
\hline Column Pitch & 0.00001 & -0.00001 & neg & neg \\
\hline CR Channel Diameter & neg & neg & neg & neg \\
\hline CR Channel Pitch & neg & neg & neg & neg \\
\hline Permanent Reflector Diameter & -0.00083 & 0.00024 & -- & -- \\
\hline Dummy Block Dimensions & \multicolumn{4}{|c|}{ See Sections 2.1.2.7 and 2.1.3.9 } \\
\hline 3.4 wt. \% Enrichment & -0.00054 & 0.00042 & -- & -- \\
\hline 3.9 wt. \% Enrichment & -0.00079 & 0.00079 & -- & -- \\
\hline 4.3 wt. $\%$ Enrichment & -0.00133 & 0.00133 & -- & -- \\
\hline 4.8 wt. $\%$ Enrichment & -0.00052 & 0.00051 & -- & -- \\
\hline 5.2 wt. \% Enrichment & -0.00039 & 0.00037 & -- & -- \\
\hline 5.9 wt. \% Enrichment & -0.00052 & 0.00047 & -- & -- \\
\hline 6.3 wt. $\%$ Enrichment & -0.00030 & 0.00026 & -- & -- \\
\hline 6.7 wt. \% Enrichment & 0.00006 & 0.00005 & -- & -- \\
\hline 7.2 wt. $\%$ Enrichment & -0.00012 & 0.00011 & -- & -- \\
\hline 7.9 wt. \% Enrichment & -0.00008 & 0.00006 & -- & -- \\
\hline 9.4 wt. \% Enrichment & 0.00006 & 0.00005 & -- & -- \\
\hline 9.9 wt. \% Enrichment & 0.00006 & 0.00007 & -- & -- \\
\hline Oxygen to Uranium Ratio & 0.00009 & -0.00012 & -- & -- \\
\hline $\mathrm{UO}_{2}$ Density & \multicolumn{4}{|c|}{ Correlated Parameter (see Section 2.1.6) } \\
\hline $\mathrm{UO}_{2}$ Impurity & 0.00047 & -0.00073 & -- & -- \\
\hline Buffer Density & neg & neg & neg & neg \\
\hline Buffer Impurity & 0.00009 & -0.00012 & -- & -- \\
\hline IPyC Density & neg & neg & neg & neg \\
\hline IPyC Impurity & 0.00013 & 0.00009 & -- & -- \\
\hline SiC Density & NA & neg & NA & neg \\
\hline SiC Impurity & neg & -0.00016 & -- & -- \\
\hline OPyC Density & neg & neg & neg & neg \\
\hline OPyC Impurity & 0.00020 & -0.00023 & -- & -- \\
\hline Overcoat Density & -0.00001 & 0.00001 & neg & neg \\
\hline Overcoat Composition & NA & NA & NA & NA \\
\hline Overcoat Impurity & 0.00032 & -0.00069 & -- & -- \\
\hline Compact Density & neg & 0.00001 & neg & neg \\
\hline Compact Impurity & 0.00009 & -0.00065 & -- & -- \\
\hline Compact Free U Content & NA & neg & -- & -- \\
\hline
\end{tabular}


NEA/NSC/DOC(2006)1

Gas Cooled (Thermal) Reactor - GCR

HTTR-GCR-RESR-002

CRIT-REAC-RRATE

Table 2.86 (cont'd.). Total Experimental Uncertainty (Case 5).

\begin{tabular}{|c|c|c|c|c|}
\hline Varied Parameter & $\begin{array}{l}\text { Systematic } \\
-\Delta \mathrm{k}_{\mathrm{eff}}(1 \sigma) \\
\end{array}$ & $\begin{array}{l}\text { Uncertainty } \\
+\Delta \mathrm{k}_{\mathrm{eff}}(1 \sigma) \\
\end{array}$ & $\begin{array}{r}\text { Random } \\
-\Delta \mathrm{k}_{\mathrm{eff}}(1 \sigma) \\
\end{array}$ & $\begin{array}{l}\text { ncertainty } \\
+\Delta \mathrm{k}_{\mathrm{eff}}(1 \sigma) \\
\end{array}$ \\
\hline Sleeve Density & -0.00005 & 0.00004 & neg & neg \\
\hline Sleeve Impurity & 0.00031 & -0.00031 & -- & -- \\
\hline BP Absorber Density & 0.00009 & -0.00011 & neg & neg \\
\hline BP Absorber Content, 2.0 wt. \% & 0.00075 & -0.00066 & 0.00004 & -0.00003 \\
\hline BP Absorber Content, 2.5 wt. \% & 0.00029 & -0.00027 & 0.00002 & -0.00002 \\
\hline BP Absorber Impurity & NA & 0.00024 & -- & -- \\
\hline BP Isotopic Abundance of ${ }^{10} \mathrm{~B}$ & 0.00136 & -0.00077 & -- & -- \\
\hline Graphite Disk Density & neg & neg & neg & neg \\
\hline Graphite Disk Impurity & neg & 0.00009 & -- & -- \\
\hline CR Absorber Density & neg & -0.00001 & neg & neg \\
\hline CR Absorber Content & 0.00002 & -0.00003 & neg & neg \\
\hline CR Absorber Impurity & NA & 0.00004 & -- & -- \\
\hline CR Isotopic Abundance of ${ }^{10} \mathrm{~B}$ & 0.00009 & 0.00021 & -- & -- \\
\hline CR Clad Density & neg & 0.00002 & neg & neg \\
\hline CR Clad Composition & 0.00009 & 0.00009 & -- & -- \\
\hline CR Clad Impurity & NA & neg & -- & -- \\
\hline Instrumentation Composition & \multicolumn{4}{|c|}{ See Section 2.1.2.6 } \\
\hline IG-110 Density in Blocks & -0.00074 & 0.00068 & -0.00010 & 0.00009 \\
\hline IG-110 Impurity in Blocks & 0.00490 & -0.00584 & -- & -- \\
\hline PGX Density & -0.00009 & 0.00006 & -0.00003 & 0.00002 \\
\hline PGX Impurity & 0.00269 & -0.00345 & -- & -- \\
\hline Dummy Block Density & 0.00002 & 0.00002 & -0.00002 & -0.00001 \\
\hline Dummy Block Impurity & 0.00217 & -0.00124 & -- & -- \\
\hline Dummy Block Type & NA & 0.00017 & NA & 0.00013 \\
\hline Helium Coolant Density & neg & neg & -- & -- \\
\hline Helium Coolant Impurity & NA & neg & -- & -- \\
\hline Room Return & neg & neg & neg & neg \\
\hline TRISO Particle Placement & 0.00068 & 0.00068 & neg & neg \\
\hline Block Stack Alignment & NA & NA & NA & NA \\
\hline MCNP Random Number Seed & -- & -- & 0.00017 & 0.00017 \\
\hline Uranium Fuel Mass (Sec. 2.1.6) & -0.00075 & 0.00073 & -0.00003 & 0.00003 \\
\hline Uncertainty of Components & 0.00675 & 0.00749 & 0.00024 & 0.00027 \\
\hline Total Evaluation Uncertainty & 0.00675 & 0.00750 & & \\
\hline
\end{tabular}


NEA/NSC/DOC(2006)1

Gas Cooled (Thermal) Reactor - GCR

HTTR-GCR-RESR-002

CRIT-REAC-RRATE

\subsection{Evaluation of Buckling and Extrapolation Length Data}

Buckling and extrapolation length measurements were not made.

\subsection{Evaluation of Spectral Characteristics Data}

Spectral characteristics measurements were not made.

\subsection{Evaluation of Reactivity Effects Data}

Evaluation of the excess reactivity measurements of the HTTR annular core configurations is provided in Section 2.4.1 of HTTR-GCR-RESR-001.

\subsection{Evaluation of Reactivity Coefficient Data}

Reactivity coefficient measurements were not made.

\subsection{Evaluation of Kinetics Measurements Data}

Kinetics measurements were not made.

\subsection{Evaluation of Reaction-Rate Distributions}

The benchmark model for the critical configuration analyzed in Section 2.1 and described in Section 3.1 was utilized in the analysis of the reactor physics experiments in Section 1.7.

Monte Carlo n-Particle (MCNP) version 5.1.40 was utilized to predict the biases and uncertainties associated with the experimental results for HTTR critical configurations in this evaluation. MCNP is a general-purpose, continuous-energy, generalized-geometry, time-dependent, coupled n-particle, Monte Carlo transport code. The Evaluated Neutron Data File library, ENDF/B-VII.0, was utilized in analysis of the benchmark model biases and uncertainties.

It should be noted that the neutron flux could not be measured directly in the core, but the fission chambers (FCs), containing ${ }^{235} \mathrm{U}$, were used to indirectly measure the flux as fission reaction rates. A fixed $\mathrm{FC}$ in one of the instrumentation columns was used to normalize the reaction-rate measurements from the FC that was moved to various positions in another column. This was to account for variation in the flux due to movement effects of the detector and its respective components.

\subsubsection{Axial Reaction Rate Distribution}

The axial fission neutron reaction-rate distribution in Figures 1.5 for the two annular 24-fuel-column cores were digitized and renormalized to allow for further comparative analysis with computational results. The digitization of the charts was performed by Chris White at the Idaho National Laboratory using Marisoft Digitizer. ${ }^{a}$ The chart is imported into the program and bound within a grid system; each individual data point is then marked and exported as numerical data. Table 2.87 and Figures 2.8 and 2.9 represent the digitized data. A comprehensive analysis of the axial neutron reaction-rate distribution in the instrumentation columns of the fully-loaded, 30-fuel-column core has already been performed

${ }^{a}$ M. Mitchell, Marisoft Digitizer Application Version 3.3, http://digitizer.sourceforge.net/, (c) 1997. 
NEA/NSC/DOC(2006)1

\section{Gas Cooled (Thermal) Reactor - GCR \\ HTTR-GCR-RESR-002 \\ CRIT-REAC-RRATE}

(Section 2.7.1 of HTTR-GCR-RESR-001). The uncertainty in the reaction-rate measurements of the fully-loaded core were used to generate an estimate of the uncertainties in the 24-fuel-column core measurements by fitting the data to a trend, as shown in Figure 2.10. The uncertainty in the normalized reaction rates is proportional to the magnitude of the ratio. There is less uncertainty in values near the peak of the neutron flux and significantly more uncertainty for lower fluxes near the edge of the core. A $\log$ trend fit is obtained to approximate the uncertainty as a function of normalized reaction rate. The uncertainties in the 24 -fuel-column core reaction rates were then estimated using this trend and provided in Table 2.87 .

Table 2.87. Axial Neutron Fission Reaction-Rate Distribution Data for the Annular 24-Fuel-Column Core Configurations.

\begin{tabular}{|c|c|c|c|c|c|c|c|c|c|c|}
\hline \multirow{2}{*}{$\begin{array}{l}\text { Data } \\
\text { Point }\end{array}$} & \multicolumn{5}{|c|}{ Configuration $3^{(a)}$} & \multicolumn{5}{|c|}{ Configuration $4^{(\mathrm{a})}$} \\
\hline & $\begin{array}{c}\text { Normalized } \\
\text { Reaction Rate }\end{array}$ & \pm & $1 \sigma$ & $1 \sigma(\%)$ & $\begin{array}{l}\text { Height } \\
(\mathrm{cm})^{(\mathrm{b})}\end{array}$ & $\begin{array}{c}\text { Normalized } \\
\text { Reaction Rate }\end{array}$ & \pm & $1 \sigma$ & $1 \sigma(\%)$ & $\begin{array}{l}\text { Height } \\
(\mathrm{cm})^{(\mathrm{b})}\end{array}$ \\
\hline 1 & 0.6946 & \pm & 0.0199 & 2.87 & 18.89 & 0.7030 & \pm & 0.0199 & 2.83 & 18.79 \\
\hline 2 & 0.7304 & \pm & 0.0198 & 2.70 & 27.86 & 0.7383 & \pm & 0.0197 & 2.67 & 28.40 \\
\hline 3 & 0.9555 & \pm & 0.0173 & 1.81 & 86.07 & 0.9523 & \pm & 0.0174 & 1.83 & 86.36 \\
\hline 4 & 1 & \pm & 0 . & 1 & 1 & 1.0000 & \pm & 6 & 1. & 79 \\
\hline 5 & 0.9854 & \pm & 0.0169 & 1.71 & 137.15 & 0.9749 & \pm & 0.0170 & 1.75 & 137.45 \\
\hline 6 & 0.9750 & \pm & 0.0170 & 1.75 & 143.96 & 0.9562 & \pm & 0.0173 & 1.81 & 144.57 \\
\hline 7 & 0.6735 & \pm & 0.0200 & 2.97 & 202.48 & 0.7915 & \pm & 0.0193 & 2.44 & 202.72 \\
\hline 8 & 0.2818 & \pm & 0.0165 & 5.86 & 260.99 & 0.5582 & \pm & 0.0201 & 3.59 & 260.85 \\
\hline 9 & 0.1041 & \pm & 0.0095 & 9.16 & 318.58 & 0.3312 & \pm & 0.0176 & 5.32 & 318.37 \\
\hline
\end{tabular}

(a) The 24-fuel-column core reaction-rate data are from the top and middle charts, respectively, in Figure 1.6.

(b) The height is in reference to the position relative to the bottom of the fifth layer of fuel. 
NEA/NSC/DOC(2006)1

Gas Cooled (Thermal) Reactor - GCR

HTTR-GCR-RESR-002

CRIT-REAC-RRATE

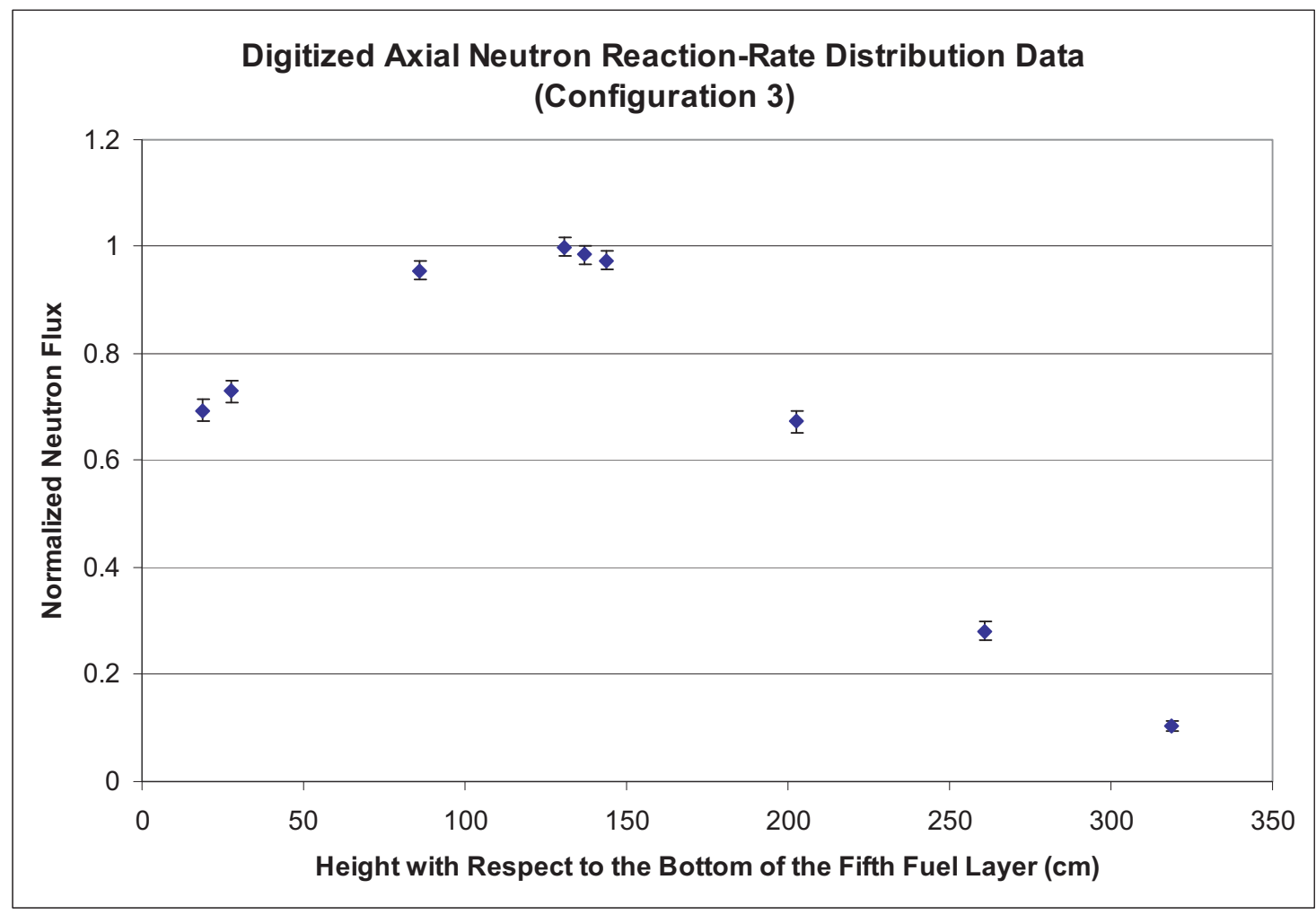

Figure 2.8. Axial Neutron Reaction-Rate Distribution for the Annular 24-Fuel-Column Core (Configuration 3).

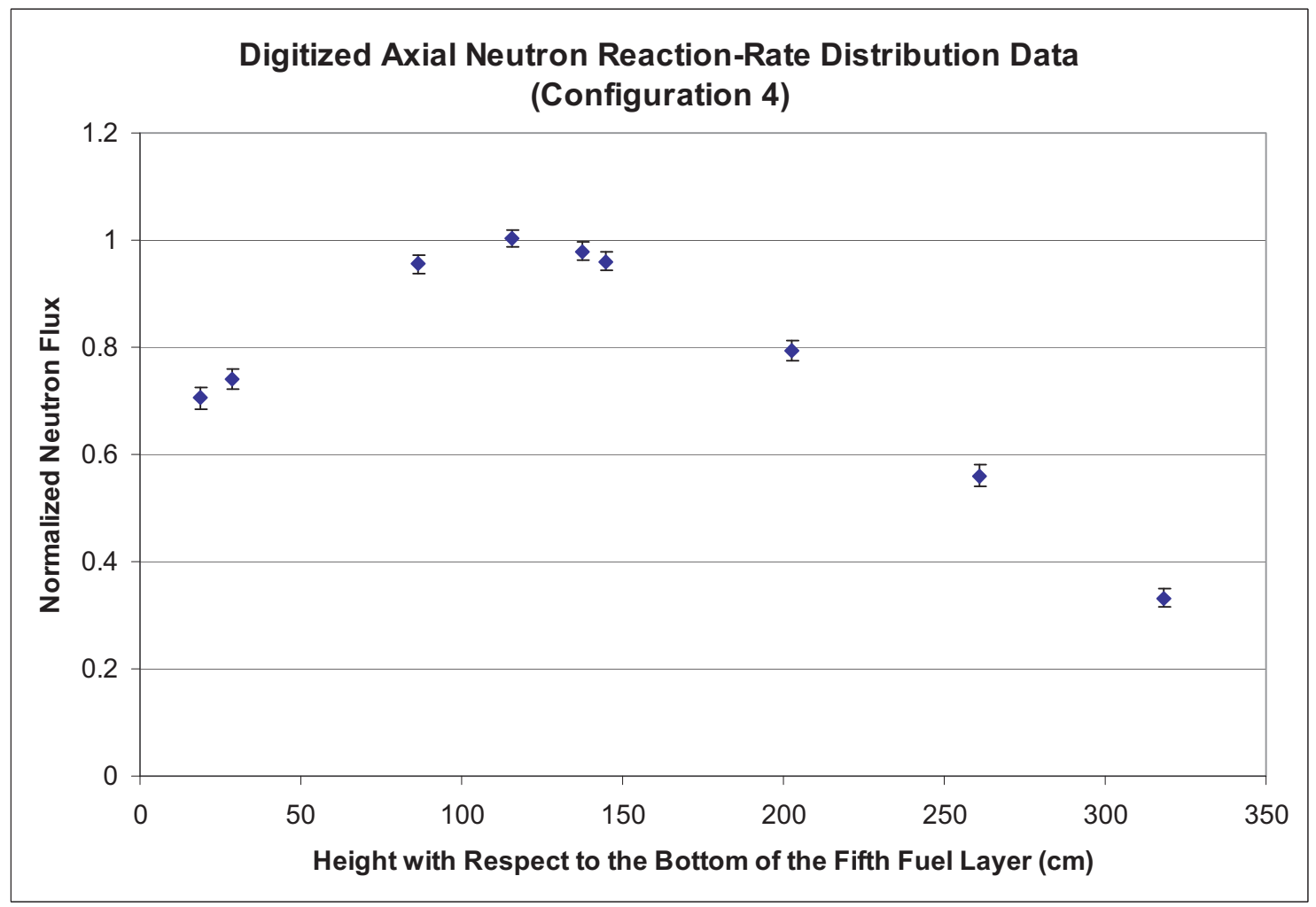

Figure 2.9. Axial Neutron Reaction-Rate Distribution for the Annular 24-Fuel-Column Core (Configuration 4). 
NEA/NSC/DOC(2006)1

Gas Cooled (Thermal) Reactor - GCR

HTTR-GCR-RESR-002

CRIT-REAC-RRATE

Estimated Uncertainty in Reaction Rate Measurements

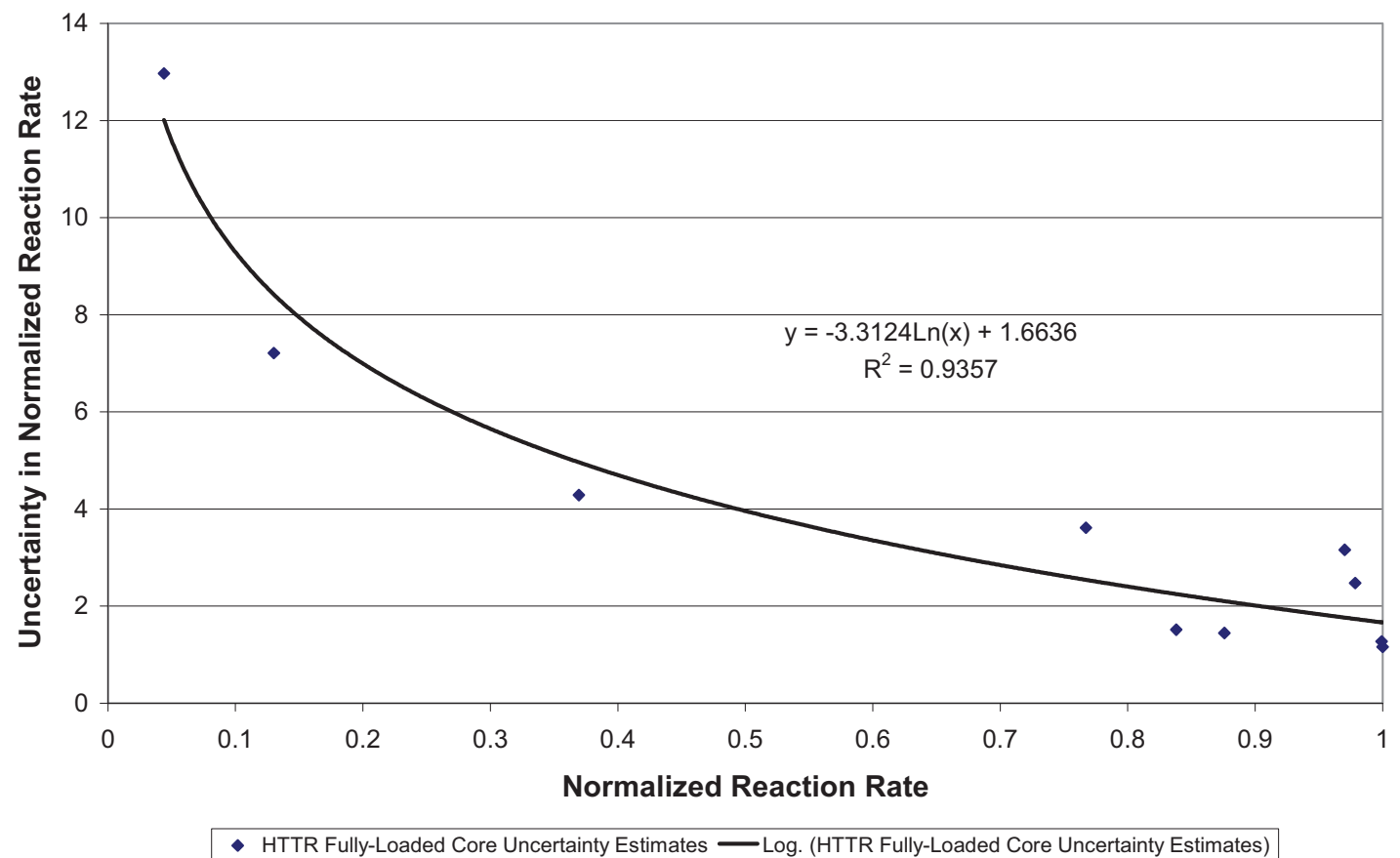

Figure 2.10. Uncertainty Trend Estimated with HTTR Fully-Loaded-Core Analysis.

The axial neutron fission reaction rate in the instrumentation columns were calculated by taking the benchmark model of the 24-fuel-column cores (configurations 3 and 4) in Section 3.1 and superimposing a flux tally over each of the instrumentation column positions: E05, E13, and E21. The flux was computed for $6.15-\mathrm{cm}$ radius discs with a thickness of $1 \mathrm{~cm}$ located at the center of one instrumentation channel in each instrumentation column (see Figure 2.11). A total of $522 \mathrm{~cm}$, representing the total height of the core fuel and reflector blocks, was modeled. The (x, y) coordinates used for columns E05, $\mathrm{E} 13$, and E21, are $(114.6005,72.4),(5.4,-135.447)$, and $(-120,63.04693)$, respectively, where the origin is located at the radial center of the core.

The F4 flux tally is used in MCNP, which determines the flux across a cell volume by tabulating the average track length of the neutrons. ${ }^{\text {a }}$ The tally is then modified by a tally multiplier card, Fm, that accounts for the total fission cross section of ${ }^{235} \mathrm{U}$, the fissile material in the fission chambers, to obtain the neutron reaction-rate in each instrumentation column.

The calculated neutron fission reaction rates are obtained by taking the variance-weighted average of results obtained using six variations of the input deck (Appendix A.1) with different random number seeds and tallies of the neutron reaction rate (Appendix A.3). This approach was used to reduce the statistical uncertainty in the neutron flux tallies because the relative error values obtained can underpredict the true uncertainty in the calculated neutron flux. ${ }^{\mathrm{b}}$ Therefore, the final calculated values are obtained from a total of 18 reaction-rate tallies ( 6 input decks with 3 instrumentation columns each).

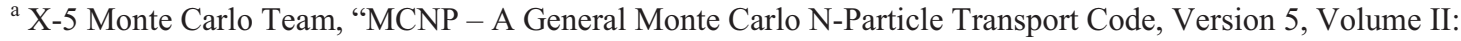
User's Guide,” LA-CP-03-0245 (April 24, 2003; revised October 2, 2005).

${ }^{b}$ F. B. Brown, “A Review of Best Practices for Monte Carlo Criticality Calculations," Proc. NCSD 2009, Richland, WA, September 13-17 (2009).
}

Revision: 0

Page 102 of 183

Date: March 31, 2010 
NEA/NSC/DOC(2006)1

Gas Cooled (Thermal) Reactor - GCR

HTTR-GCR-RESR-002

CRIT-REAC-RRATE

The average of the neutron reaction rate in each position is taken and normalized to represent the calculated axial neutron reaction-rate profile.

$$
\phi(z)_{\text {normalized }}=\frac{\phi(z)}{\phi_{\text {maximum }}},
$$

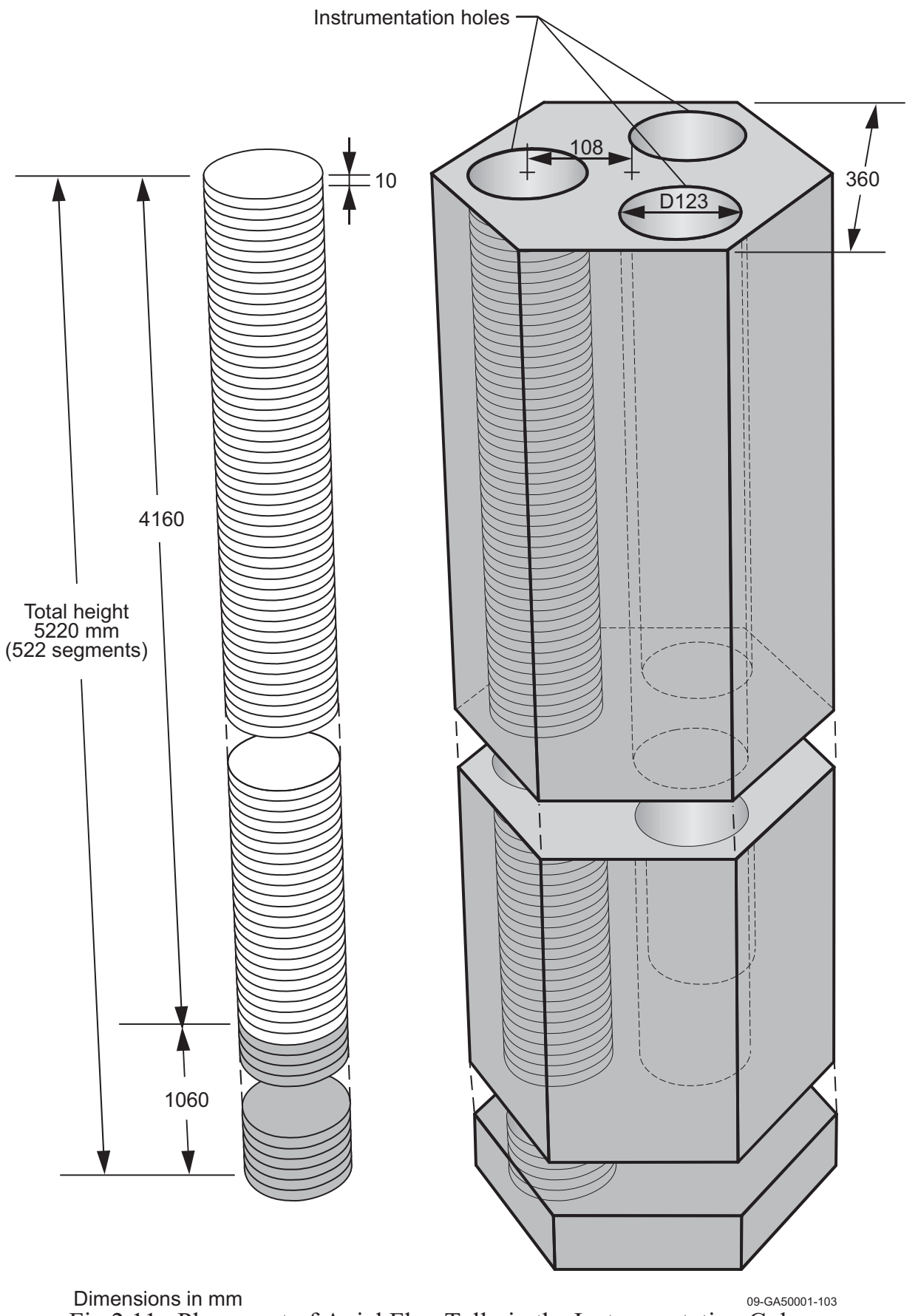

Fig 2.11. Placement of Axial Flux Tally in the Instrumentation Column. 
NEA/NSC/DOC(2006)1

Gas Cooled (Thermal) Reactor - GCR

HTTR-GCR-RESR-002

CRIT-REAC-RRATE

\subsection{Evaluation of Power Distribution Data}

Power distribution measurements were not made.

\section{$2.9 \quad$ Evaluation of Isotopic Measurements}

Isotopic measurements were not made.

\subsection{Evaluation of Other Miscellaneous Types of Measurements}

Other miscellaneous types of measurements were not made. 
NEA/NSC/DOC(2006)1

Gas Cooled (Thermal) Reactor - GCR

HTTR-GCR-RESR-002

CRIT-REAC-RRATE

\subsection{BENCHMARK SPECIFICATIONS}

\subsection{Benchmark-Model Specifications for Critical and / or Subcritical Measurements}

Whereas insufficient information is publicly available, a finely-detailed benchmark model could not be established. A benchmark of the HTTR was prepared and analyzed with as much detail as feasible. The simplification bias for this model could also not be fully determined. However, the uncertainties in the benchmark model are believed to be of sufficient magnitude to encompass any biases incurred due to the simplification process of the benchmark model and a bias for the removal of the core instrumentation has been estimated. It is currently difficult to obtain the necessary information to improve the confidence in the benchmark model; the necessary data is proprietary and its released is being restricted, because the benchmark configuration of the HTTR core is the same that is currently in operation. Once this information is made available, the HTTR benchmark can be adjusted as appropriate.

Models for all five configurations of the annular HTTR core are provided. The five configurations, or cases, in this benchmark analysis use four core layouts, with the third core layout implementing two different control rod positionings. Configuration 1 has 19 fuel columns. Configuration 2 has 21 fuel columns. Configurations 3 and 4 have 24 fuel columns. Configuration 5 has 27 fuel columns. Analysis of the fully-loaded, 30-fuel-column, core can be found in HTTR-GCR-RESR-001.

\subsubsection{Description of the Benchmark Model Simplifications}

Significant simplifications were incurred to develop a benchmark model of the HTTR because of a lack of information publicly available to determine dimensions and compositions. Simplifications will be discussed where applicable in the descriptions of the dimension and material properties of the model.

As stated previously, biases of the model were not assessed but will be addressed as additional HTTR information becomes available. Biases that have been partially investigated are listed in Section 3.1.1.1.

The fuel handling positions, dowels, and sockets were not included in the model due to insufficient data specifications, but were accounted for with a void fraction of $0.5 \%$ reduction in graphite density (based upon volume calculations using dimensions provided in Figure 1.52 of HTTR-GCR-RESR-001). The burnable poison insertion holes were placed on the same pitch as the fuel channels to simplify the model.

It is apparent from a comparison of Figures 1.65 and 1.67 of HTTR-GCR-RESR-001 that the depth to which the control rod, reserve shutdown system, and instrumentation holes are drilled varies. A depth of $1060 \mathrm{~mm}$ above the bottom of the core was selected for all positions to simplify the model. No bias was assessed.

Insufficient information was available to model the bottom-most reflector block according to actual design; therefore it was modeled with the same design as the two top reflector blocks and the other bottom reflector block. The top and bottom of each coolant channel is expected to taper from the $21-\mathrm{mm}$ diameter to the $41-\mathrm{mm}$ diameter of the fuel assemblies, but information was unavailable to describe the taper in the model. Therefore channels in the reflector blocks were modeled with 21 -mm diameters.

Individual sections of the dodecagon-block-shaped permanent reflector were not modeled due to insufficient information. It was modeled as a cylindrical region surrounding the core columns. A bias could not be assessed.

Insufficient information was available to model the shielding blocks surrounding the core and shielding plugs in the core. Therefore, they were not included in the benchmark model. It is assumed that all neutrons reaching the core boundaries are lost and not scattered back by the shielding material. A conservative estimate of room-return effects demonstrated a negligible change in $\mathrm{k}_{\text {eff. }}$. 
NEA/NSC/DOC(2006)1

\section{Gas Cooled (Thermal) Reactor - GCR \\ HTTR-GCR-RESR-002 \\ CRIT-REAC-RRATE}

In the materials section, impurity contents in the materials are based upon natural boron equivalency. In the model, however, only the ${ }^{10} \mathrm{~B}$ component is included, as the effect of the ${ }^{11} \mathrm{~B}$ content would be insignificant.

The density is the same $\left(1.80 \mathrm{~g} / \mathrm{cm}^{3}\right)$ for both types of burnable poison pellets. The boron content in the pellets is based on the reported weight percents instead of the atomic percents.

Partially-withdrawn control rod positions represent the average height of the values reported in Table 1.1, which are reported in Table 1.2 except for configuration 4. The average height for the partiallywithdrawn rods in configuration 4 is taken to be $1592 \mathrm{~mm}$. Fully-withdrawn rod positions are $4050 \mathrm{~mm}$ for all rods except those in the R2 positions; their maximum withdrawal height is $3325 \mathrm{~mm}$.

Too much information was available to specify parameters for the TRISO particle fuel. Because the fuel mass of an individual rod would most probably be the most accurate measured parameter, it was preserved in the benchmark model with some variation to other parameters as necessary. The TRISO kernel diameter is maintained at the nominal value of $600 \mu \mathrm{m}$ and the density of the fuel is $10.39 \mathrm{~g} / \mathrm{cm}^{3}$, which is within approximately $95 \%$ of the theoretical density of $\mathrm{UO}_{2}$. The number of TRISO particles in a given compact was reduced from 13,000 to 12,987 , with a packing fraction of $30 \%$, in order to conserve a nominal fuel mass per rod of $188.58 \mathrm{~g}$.

\subsubsection{Assessed Biases}

Although some biases have been partially investigated, there is incomplete information regarding the HTTR to properly address simplification biases in order to adjust the benchmark $\mathrm{k}_{\mathrm{eff}}$. As stated previously, a conservative estimate of potential room-return effects provided negligible results. As shown in Section 2.1.3.2 in Table 2.52, the effect of neglecting the free uranium content of the fuel compacts was negligible. Finally, the effect of modeling the helium coolant as void material was also negligible (as shown in Section 2.1.3.10, Table 2.77). The reported literature bias for air content in the graphite could not be verified (Section 2.1.3.7).

Previous efforts of the Japanese in analyzing the 19-fuel-column core (Case 1) obtained an analytical excess reactivity of $2.7 \% \Delta \mathrm{k} / \mathrm{k}$, with an estimated Monte Carlo calculation overestimate of $1.2 \% \Delta \mathrm{k} / \mathrm{k}$. Additional information would be necessary to completely verify published results

An approximate bias for the removal of reactor instrumentation from the three instrumentation columns in the core was calculated, as discussed in Section 2.1.2.6. Calculated biases with uncertainties are shown in Table 3.1.

\footnotetext{
${ }^{a}$ Fujimoto, N., Nakano, M., Takeuchi, M., Fujisaki, S., and Yamashita, K., "Start-Up Core Physics Tests of High Temperature Engineering Test Reactor (HTTR), (II): First Criticality by an Annular Form Fuel Loading and Its Criticality Prediction Method," J. Atomic Energy Society Japan, 42(5), 458-464 (2000).
}

Revision: 0

Page 106 of 183

Date: March 31, 2010 
NEA/NSC/DOC(2006)1

Gas Cooled (Thermal) Reactor - GCR

HTTR-GCR-RESR-002

CRIT-REAC-RRATE

Table 3.1. Estimated Bias for the Removal of Instrumentation Components.

\begin{tabular}{||c|ccc||}
\hline Case & $\Delta \mathrm{k}$ & \pm & $\sigma_{\Delta \mathrm{k}}$ \\
\hline \hline 1 & 0.00483 & \pm & 0.00139 \\
\hline 2 & 0.00403 & \pm & 0.00116 \\
\hline 3 & 0.00348 & \pm & 0.00100 \\
\hline 4 & 0.00315 & \pm & 0.00091 \\
\hline 5 & 0.00291 & \pm & 0.00084 \\
\hline
\end{tabular}

\subsubsection{Dimensions}

\subsubsection{Prismatic Pin-in-Block Fuel}

\section{TRISO Particles}

The basic ingredient for HTTR fuel is the TRISO particle. A $\mathrm{UO}_{2}$ kernel is surrounded by four coatings: a low density porous pyrolytic carbon (PyC) buffer layer, a high density inner isotropic PyC layer, a SiC layer, and a final outer PyC layer. A resinated graphite overcoat is then deposited around each TRISO particle. Figure 3.1 depicts the TRISO layers and their respective dimensions.

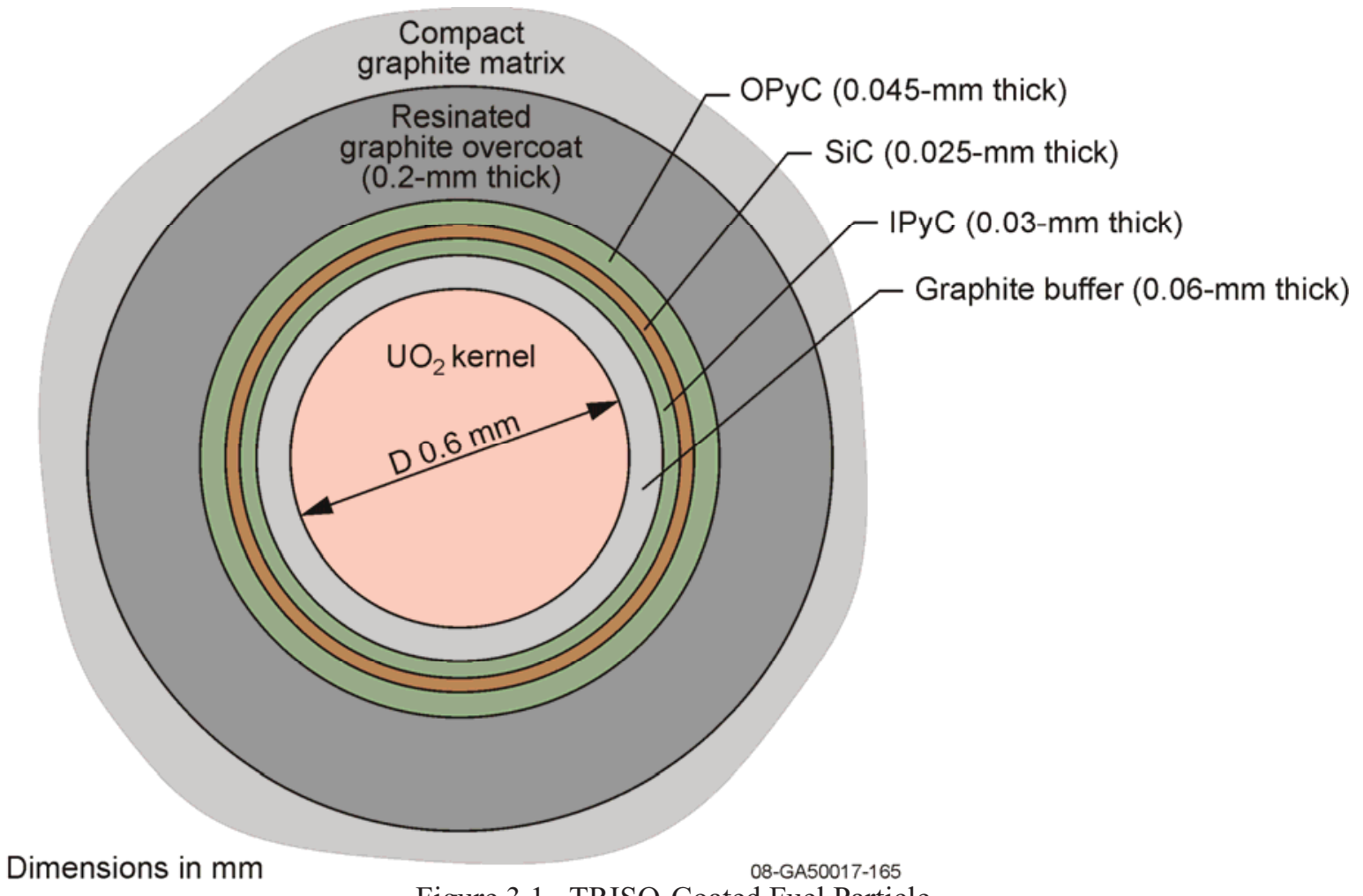

Figure 3.1. TRISO-Coated Fuel Particle. 
NEA/NSC/DOC(2006)1

\section{Gas Cooled (Thermal) Reactor - GCR \\ HTTR-GCR-RESR-002 \\ CRIT-REAC-RRATE}

\section{Compacts}

All fourteen fuel compacts in a single fuel pin are modeled as a single unit filled with the TRISO lattice. The stacked compacts have an inner diameter of $1 \mathrm{~cm}$, an outer diameter of $2.6 \mathrm{~cm}$, and an overall height of $54.6 \mathrm{~cm}$.

A horizontal cross section of the compacts is shown in Figure 3.2. In the benchmark model, 12,987 TRISO particles are randomly distributed throughout the compact matrix in a single compact. For a stack of 14 compacts, as modeled in this benchmark, the total number of TRISO particles is 181,818 .

A key parameter is that the total fuel mass of a single fuel rod (14 stacked compacts) is approximately $188.58 \mathrm{~g}$.

While the benchmark model retains randomness in distribution, many computer codes cannot properly model such configurations. It is up to the user to determine which method is most appropriate while accounting for its impact on the reactivity of the model. Example means for analyzing this model are provided in Section 4.1. The difference in methods for accurately modeling random TRISO particles in a full-core reactor has been discussed in Section 2.1.4.2.

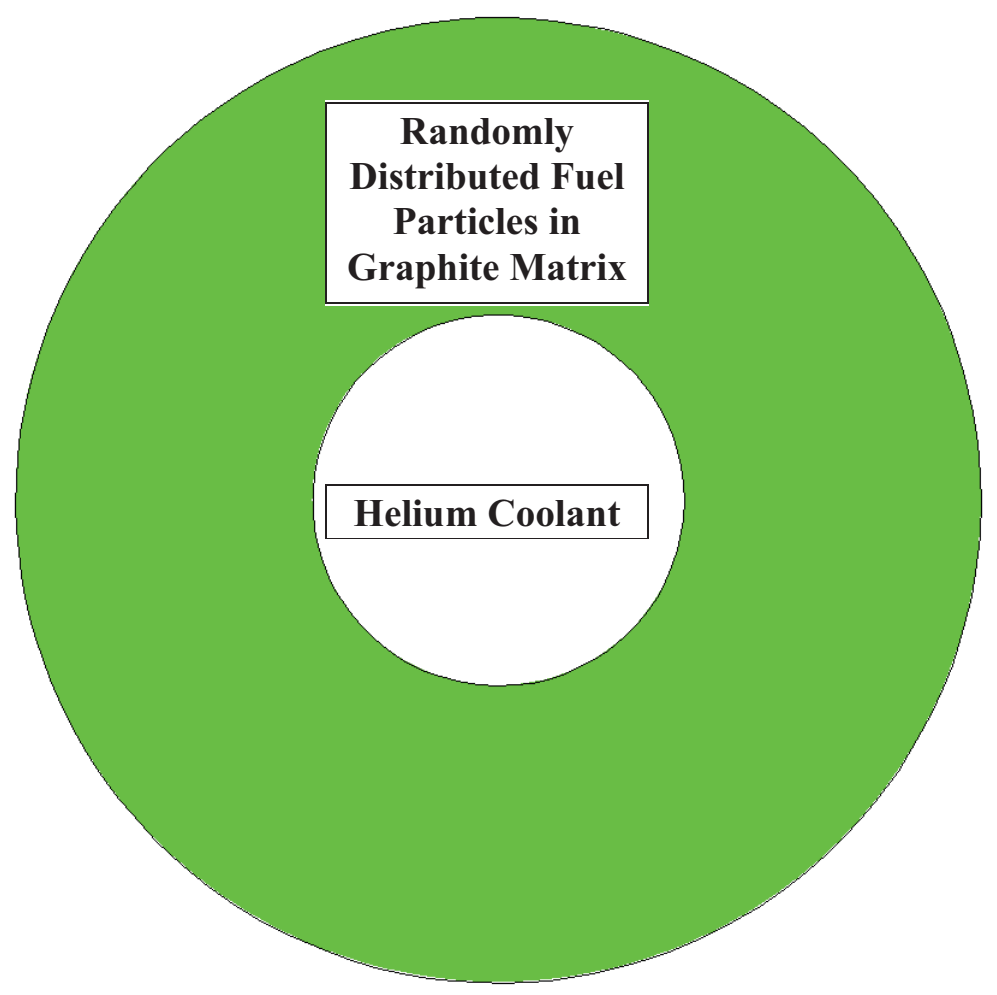

Figure 3.2. Fuel Compact Filled with Randomly Distributed TRISO Particles (Particles Not Shown). 
NEA/NSC/DOC(2006)1

Gas Cooled (Thermal) Reactor - GCR

HTTR-GCR-RESR-002

CRIT-REAC-RRATE

\section{Fuel Element}

A description of the HTTR fuel element is modeled (Figure 3.3).

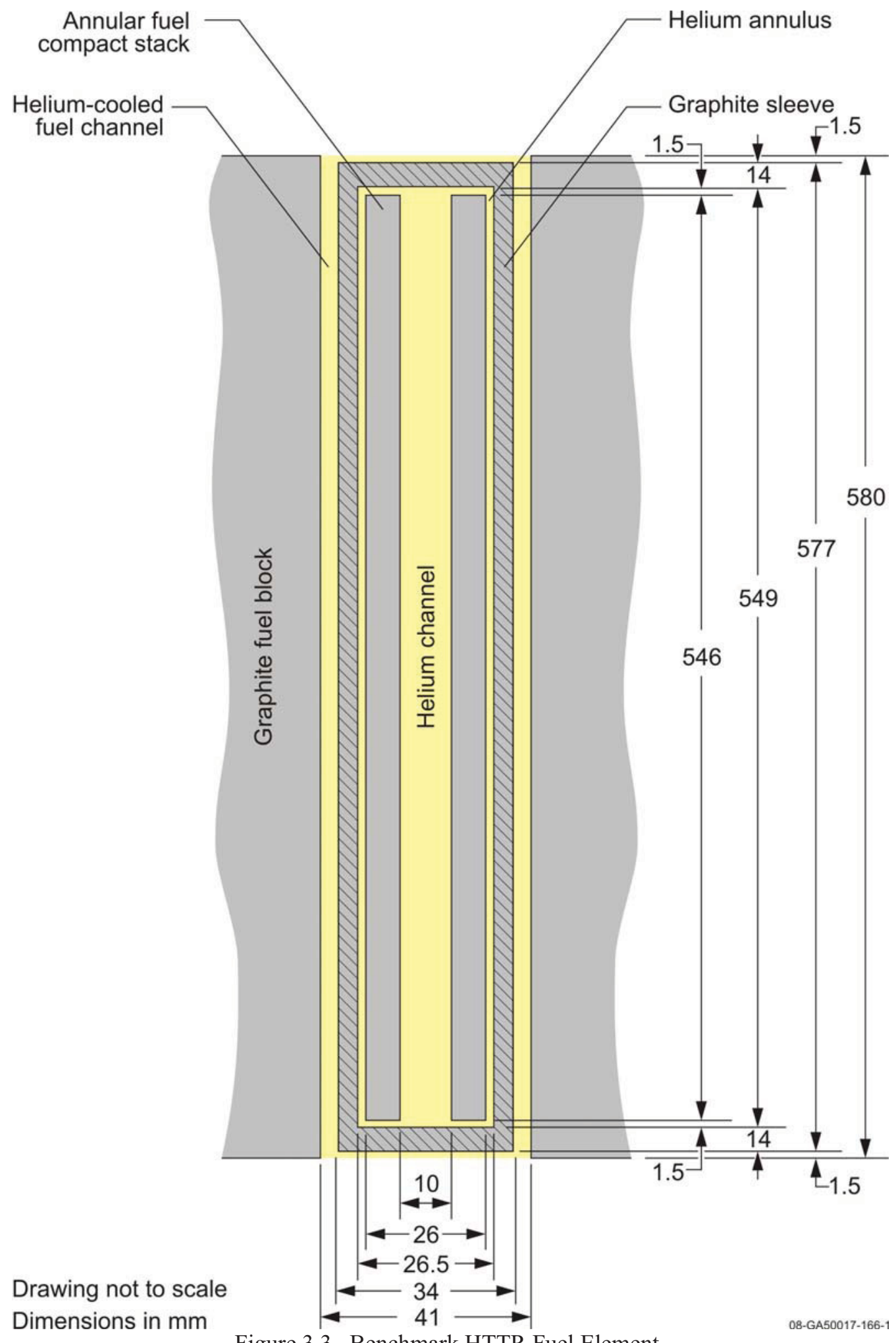

Figure 3.3. Benchmark HTTR Fuel Element. 
NEA/NSC/DOC(2006)1

Gas Cooled (Thermal) Reactor - GCR

HTTR-GCR-RESR-002

CRIT-REAC-RRATE

\subsubsection{Burnable Poisons}

The burnable poison pellets and graphite disks were modeled as individual stacks contained within a pin position in the fuel blocks (Figure 3.4). Each fuel block contained two BP pins and one empty pin position.
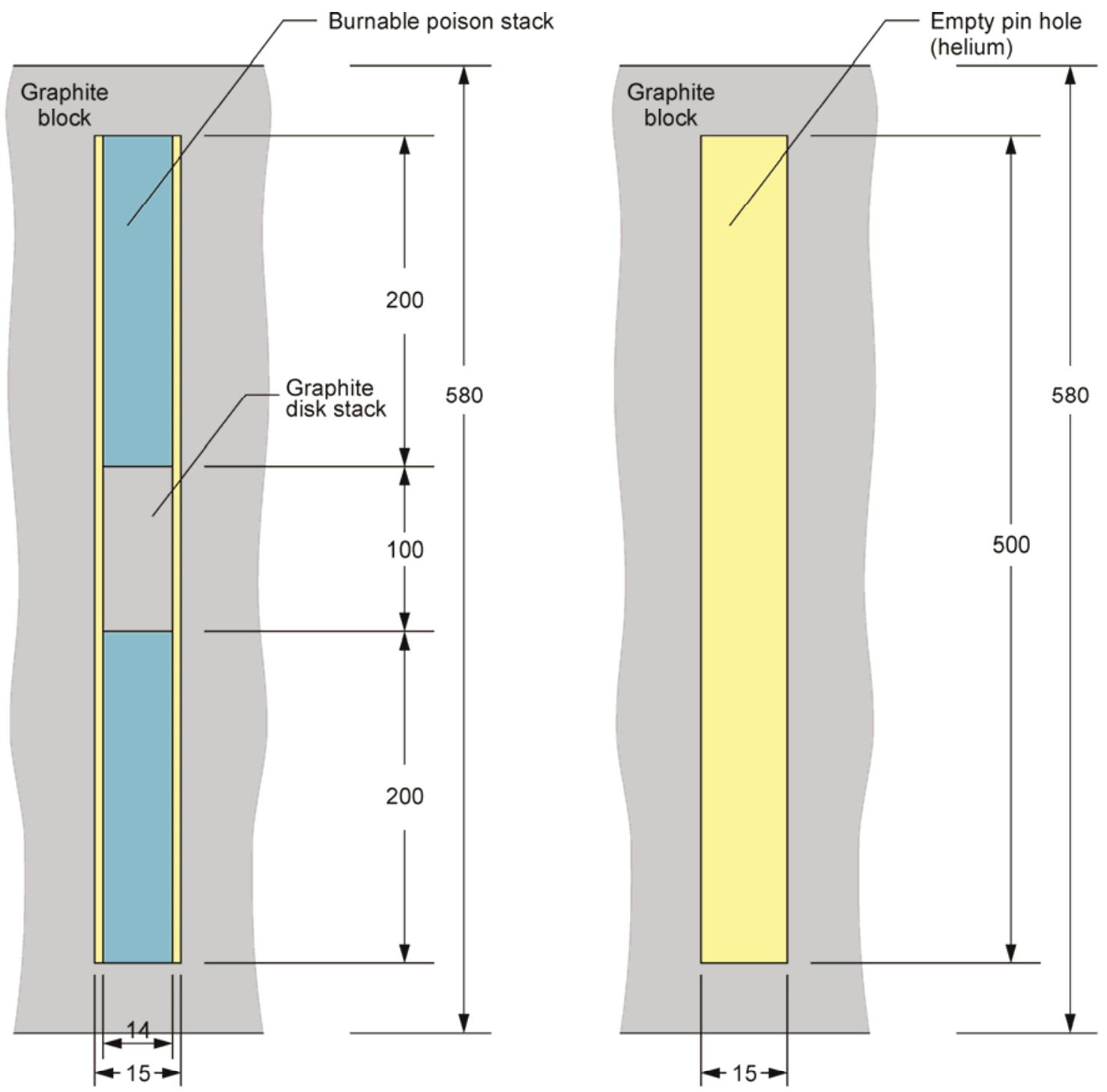

Drawing not to scale

Dimensions in $\mathrm{mm}$

Figure 3.4. Burnable Poison Pin (Left) and Empty Pin Position (Right).

08-GA50017-166-2

\subsubsection{Fuel Blocks}

The HTTR contains two types of regular hexagonal fuel blocks: 33-pin (Zones 1 and 2) and 31-pin (Zones 3 and 4). Diagrams of each fuel block design implemented in the benchmark model are shown in Figures 3.5 and 3.6, respectively. The pitch for all positions is $51.5 \mathrm{~mm}$. 
NEA/NSC/DOC(2006)1

Gas Cooled (Thermal) Reactor - GCR

HTTR-GCR-RESR-002

CRIT-REAC-RRATE

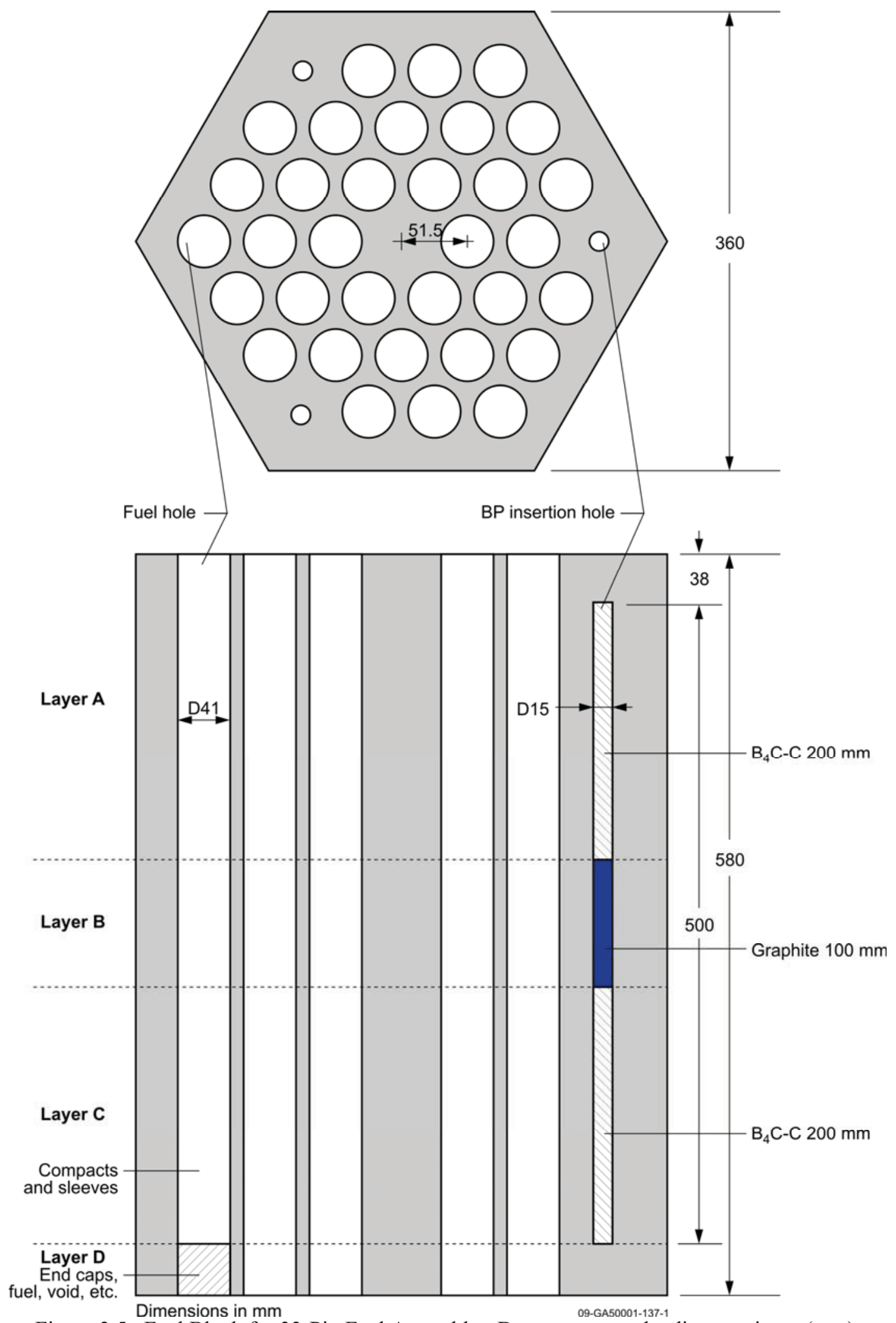

Figure 3.5. Fuel Block for 33-Pin Fuel Assembly. Dxx represents the diameter in xx (mm). 
NEA/NSC/DOC(2006)1

Gas Cooled (Thermal) Reactor - GCR

HTTR-GCR-RESR-002

CRIT-REAC-RRATE
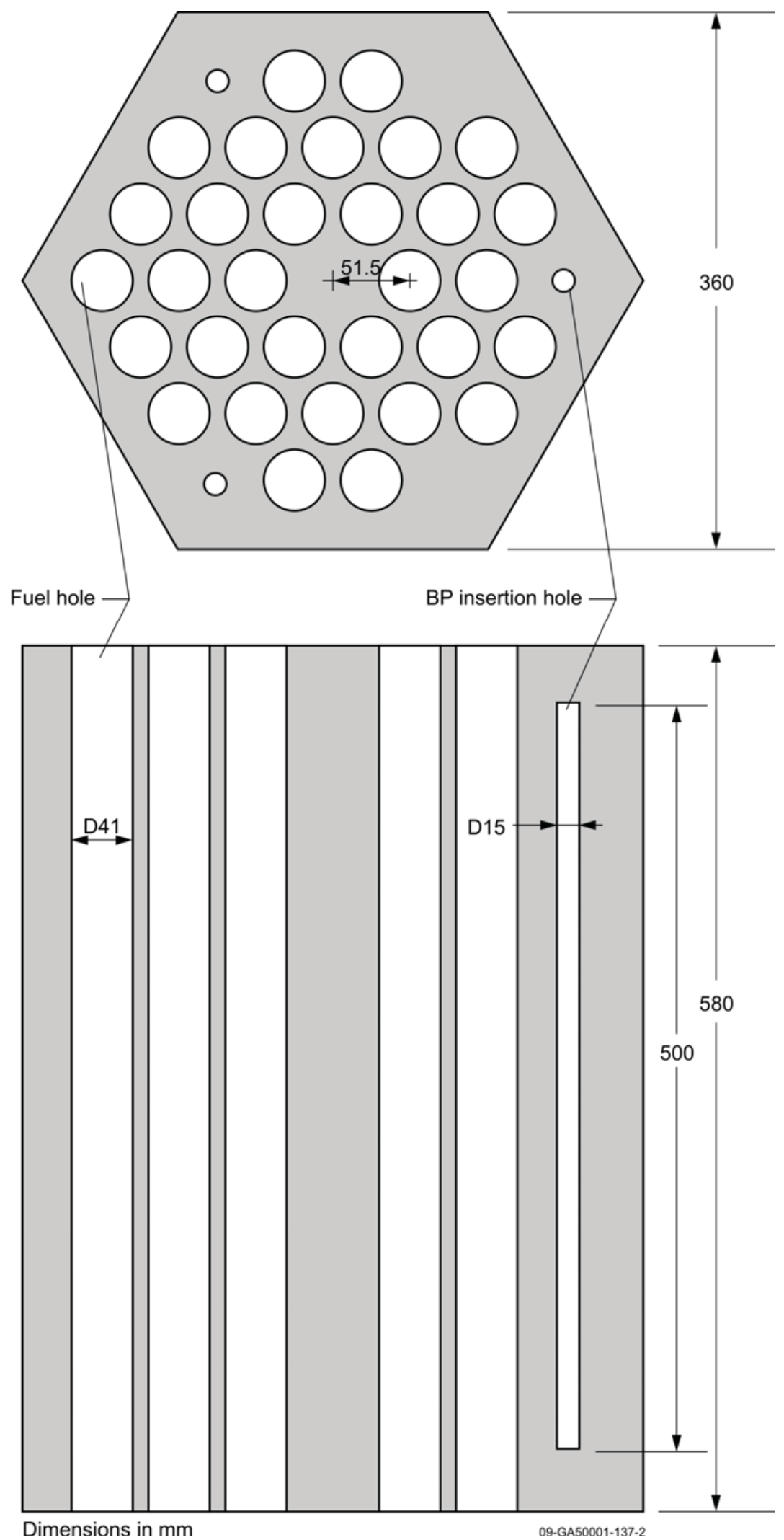

Figure 3.6. Fuel Block for 31-Pin Fuel Assembly. Dxx represents the diameter in xx (mm). 
NEA/NSC/DOC(2006)1

Gas Cooled (Thermal) Reactor - GCR

HTTR-GCR-RESR-002

CRIT-REAC-RRATE

\subsubsection{Dummy Blocks}

The HTTR contains regular hexagonal dummy blocks. A diagram of the dummy block design implemented in the benchmark model is shown in Figure 3.7.
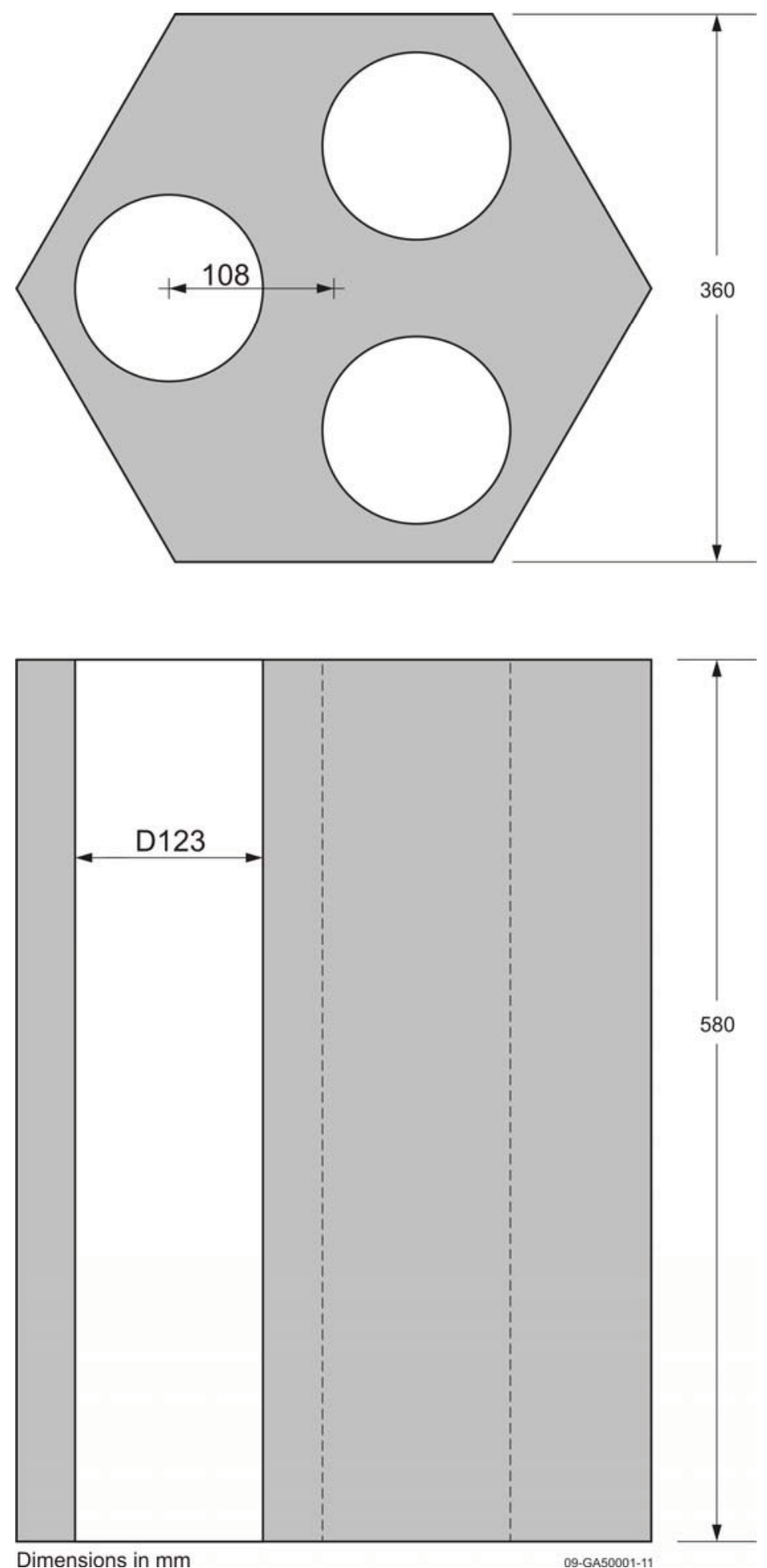

Dimensions in mm

Figure 3.7. Dummy Block. Dxx represents the diameter in $\mathrm{xx}(\mathrm{mm})$. 
NEA/NSC/DOC(2006)1

Gas Cooled (Thermal) Reactor - GCR

HTTR-GCR-RESR-002

CRIT-REAC-RRATE

\subsubsection{Control Rod System}

\section{Control Rods}

A diagram of a control rod section is shown in Figure 3.8. The absorber compacts are modeled as a single unit. Detailed dimensions regarding the cladding infrastructure for each section was unavailable, and the clad is therefore modeled without detail. A single control rod is comprised of ten sections (Figure 3.9) with a total height of $3.1 \mathrm{~m}$.

The control rods are divided up into four sets: center position (C), ring 1 (R1), ring 2 (R2), and ring 3 (R3). The center position contains two control rods. The other rings are comprised of six, six, and three positions, containing a total of twelve, twelve, and six control rods, respectively. Control rods in each set are synchronously moved.

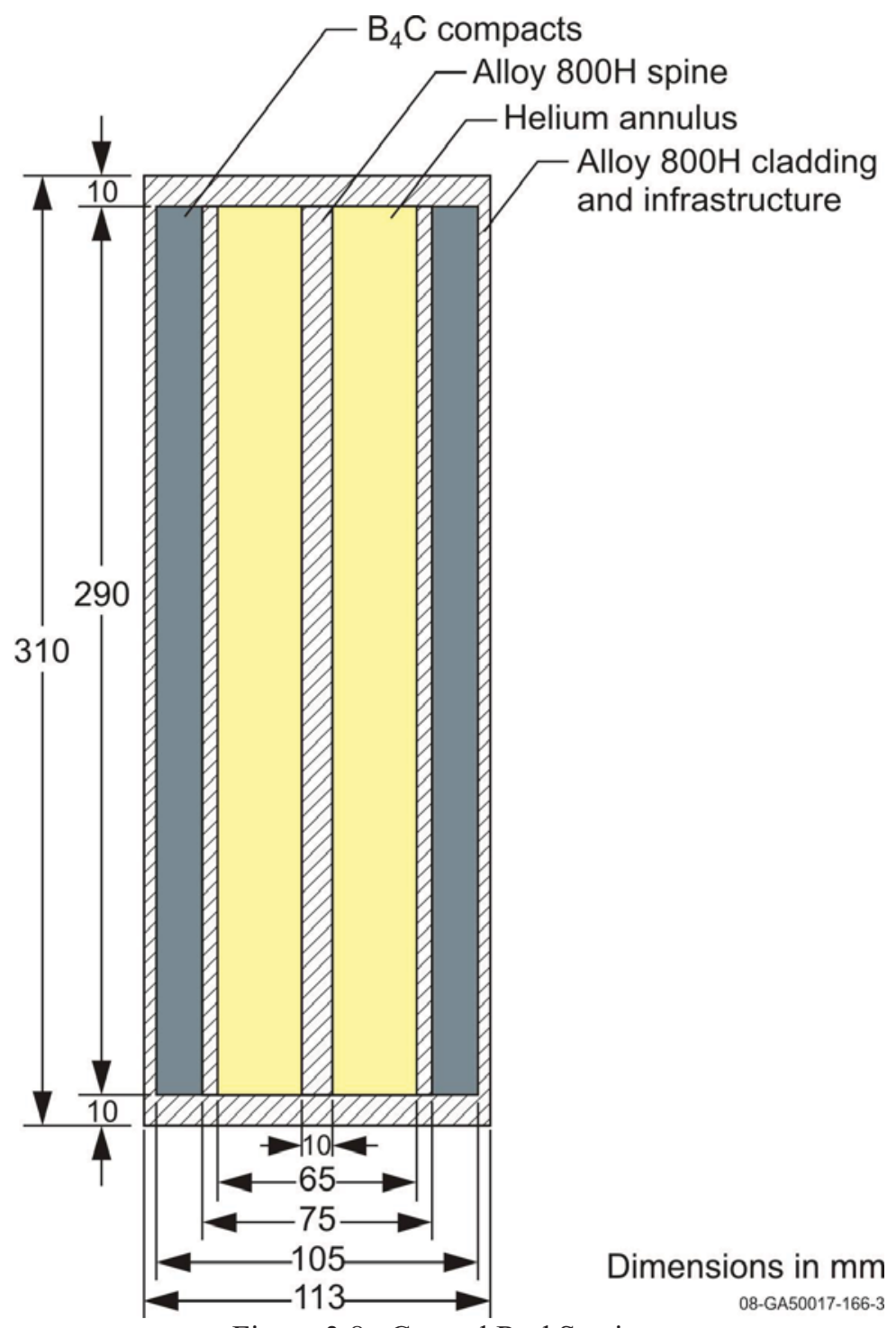

Figure 3.8. Control Rod Section. 
NEA/NSC/DOC(2006)1

Gas Cooled (Thermal) Reactor - GCR

HTTR-GCR-RESR-002

CRIT-REAC-RRATE

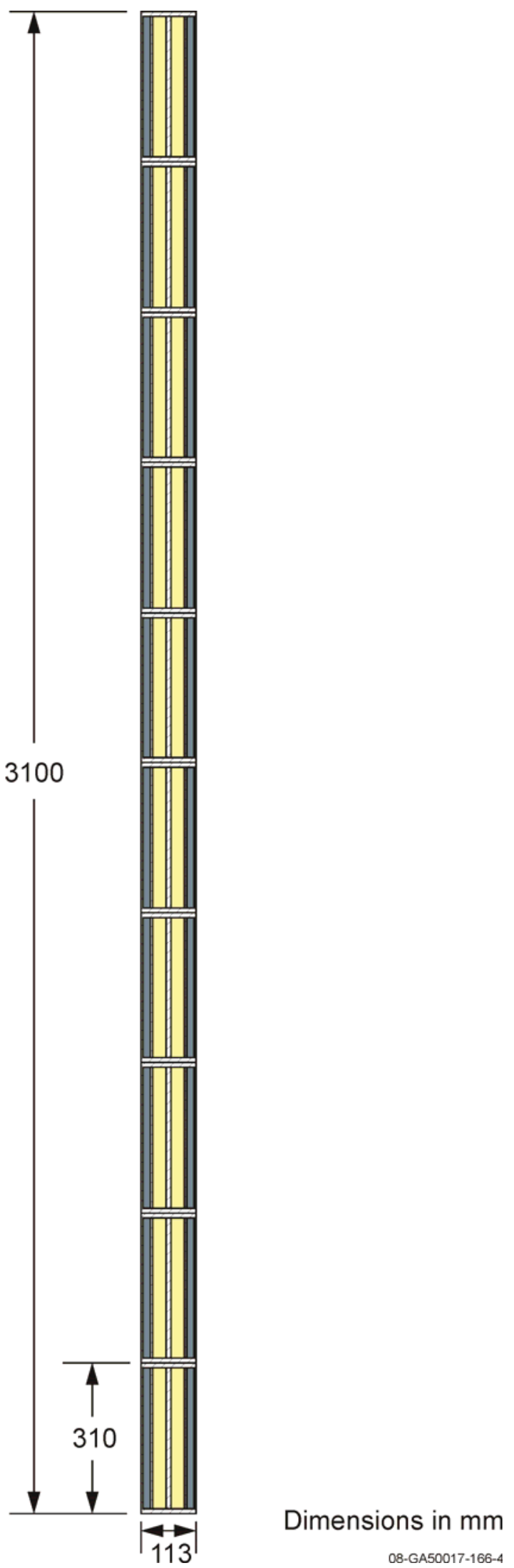

Figure 3.9. Control Rod Comprised of Ten Sections. 
NEA/NSC/DOC(2006)1

Gas Cooled (Thermal) Reactor - GCR

HTTR-GCR-RESR-002

CRIT-REAC-RRATE

\section{Control Rod Columns}

Individual control rod blocks were not modeled. A single control rod column was modeled with three holes to accommodate two control rods and an empty position (for the reserved shutdown system). A diagram of a generic control column (without control rods) is shown in Figure 3.10. The holes in the control rod and instrumentation columns are equidistant from each other, with an angle of $120^{\circ}$.
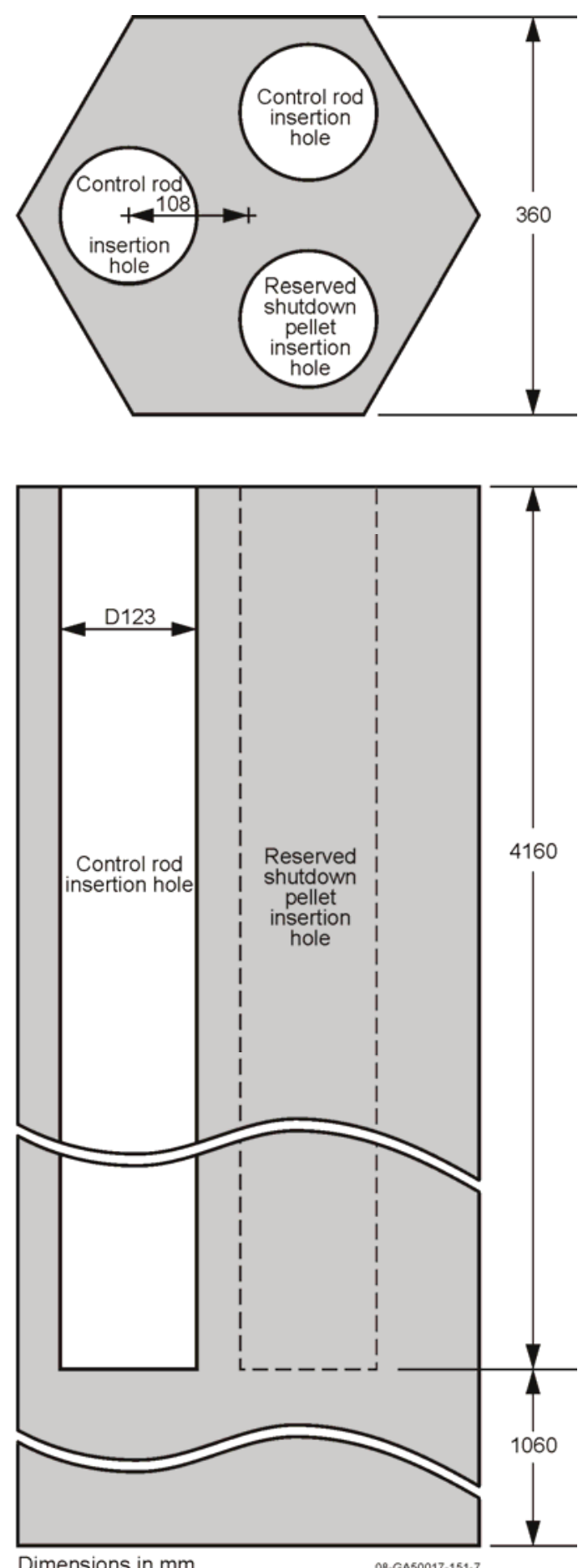

Figure 3.10. Control Rod Column. Dxx represents the diameter in xx (mm). 
NEA/NSC/DOC(2006)1

Gas Cooled (Thermal) Reactor - GCR

HTTR-GCR-RESR-002

CRIT-REAC-RRATE

\subsubsection{Instrumentation}

\section{$\underline{\text { Instrumentation Components }}$}

Instrumentation was not included in the benchmark model of the HTTR. An approximate bias with uncertainty was determined applied to the benchmark model (see Sections 2.1.2.6 and 3.1.1.1).

\section{Instrumentation Columns}

Instrumentation columns are modeled as a single unit without blocks, similar to the control rod columns. However, all three positions are empty (Figure 3.11).
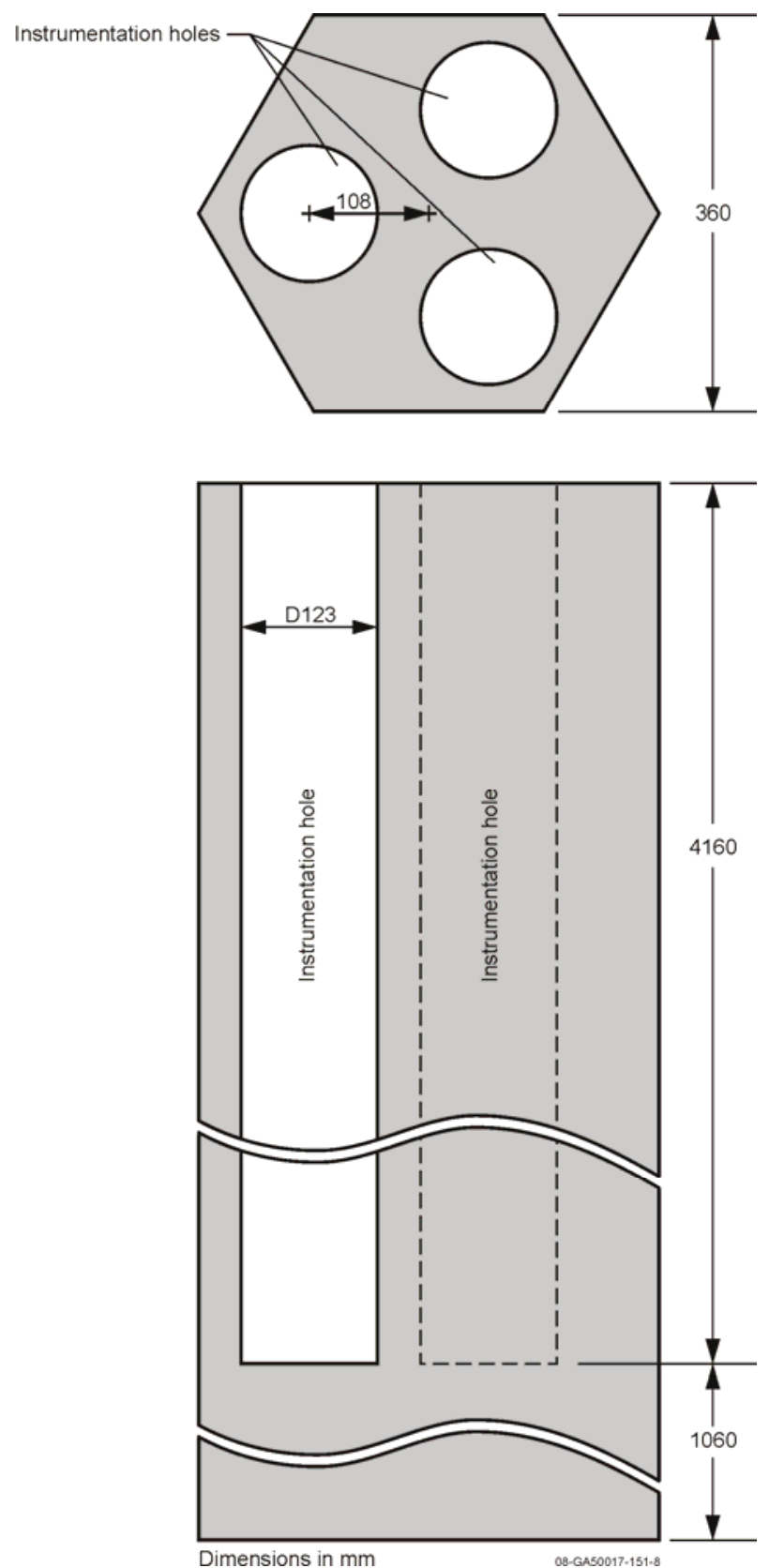

Figure 3.11. Instrumentation Column. Dxx represents the diameter in xx (mm). 
NEA/NSC/DOC(2006)1

Gas Cooled (Thermal) Reactor - GCR

HTTR-GCR-RESR-002

CRIT-REAC-RRATE

\subsubsection{Replaceable Reflector Columns}

The replaceable reflector columns are modeled as a solid unit and not as individual blocks, similar to the control rod and instrumentation columns but without any channels (Figure 3.12).
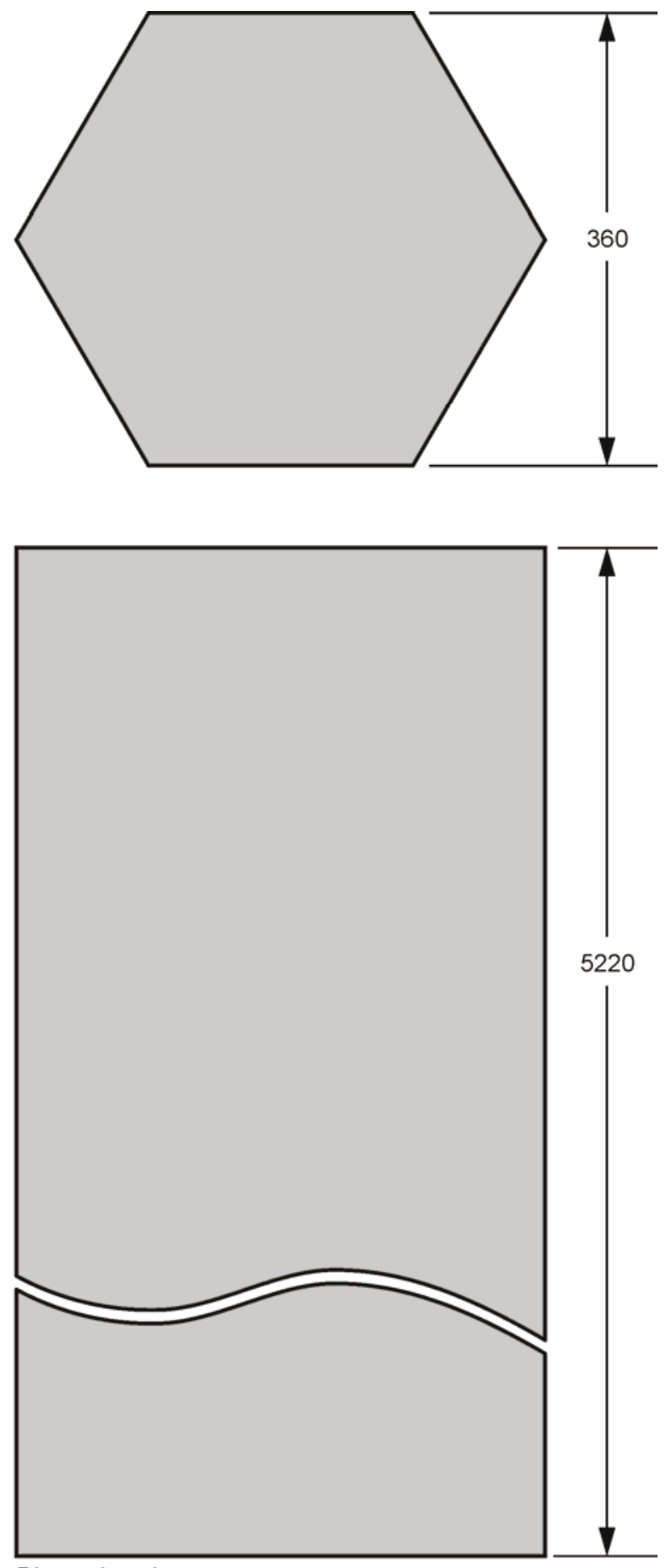

Dimensions in $\mathrm{mm}$

8.GA50017.151-11

Figure 3.12. Replaceable Reflector Column. 
NEA/NSC/DOC(2006)1

Gas Cooled (Thermal) Reactor - GCR

HTTR-GCR-RESR-002

CRIT-REAC-RRATE

\subsubsection{Replaceable Reflectors Blocks in Fuel Columns}

The replaceable reflector blocks, located at the top and the bottom fuel columns, are shown in Figures 3.13 and 3.14, for the 33-pin and 31-pin fuel assemblies, respectively. The replaceable reflector blocks have the same regular hexagonal shape and pitch as described for the fuel blocks.
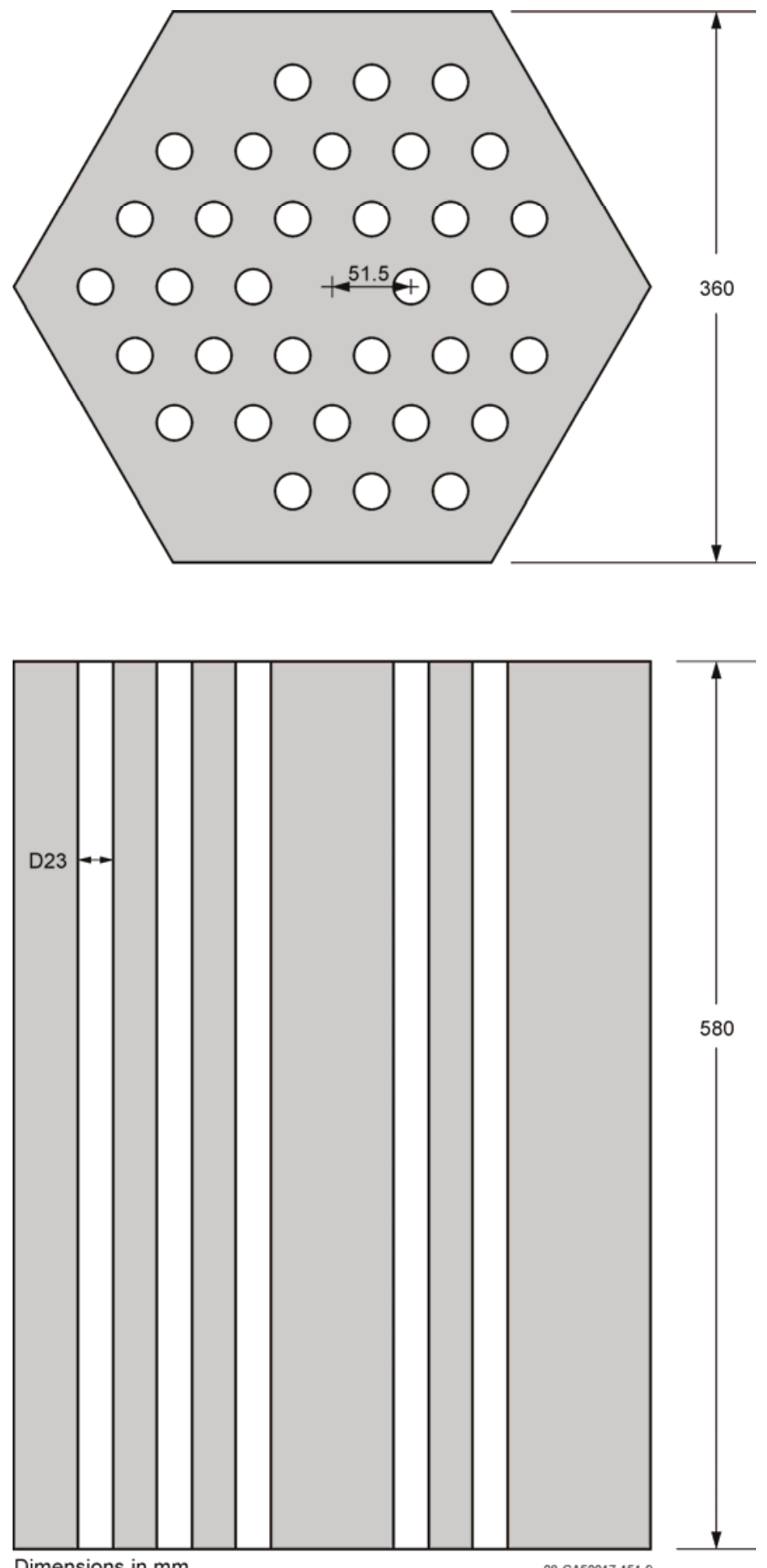

Figure 3.13. Replaceable Reflector Block for 33-Pin Fuel Assembly. Dxx represents the diameter in $\mathrm{xx}(\mathrm{mm})$. 
NEA/NSC/DOC(2006)1

Gas Cooled (Thermal) Reactor - GCR

HTTR-GCR-RESR-002

CRIT-REAC-RRATE
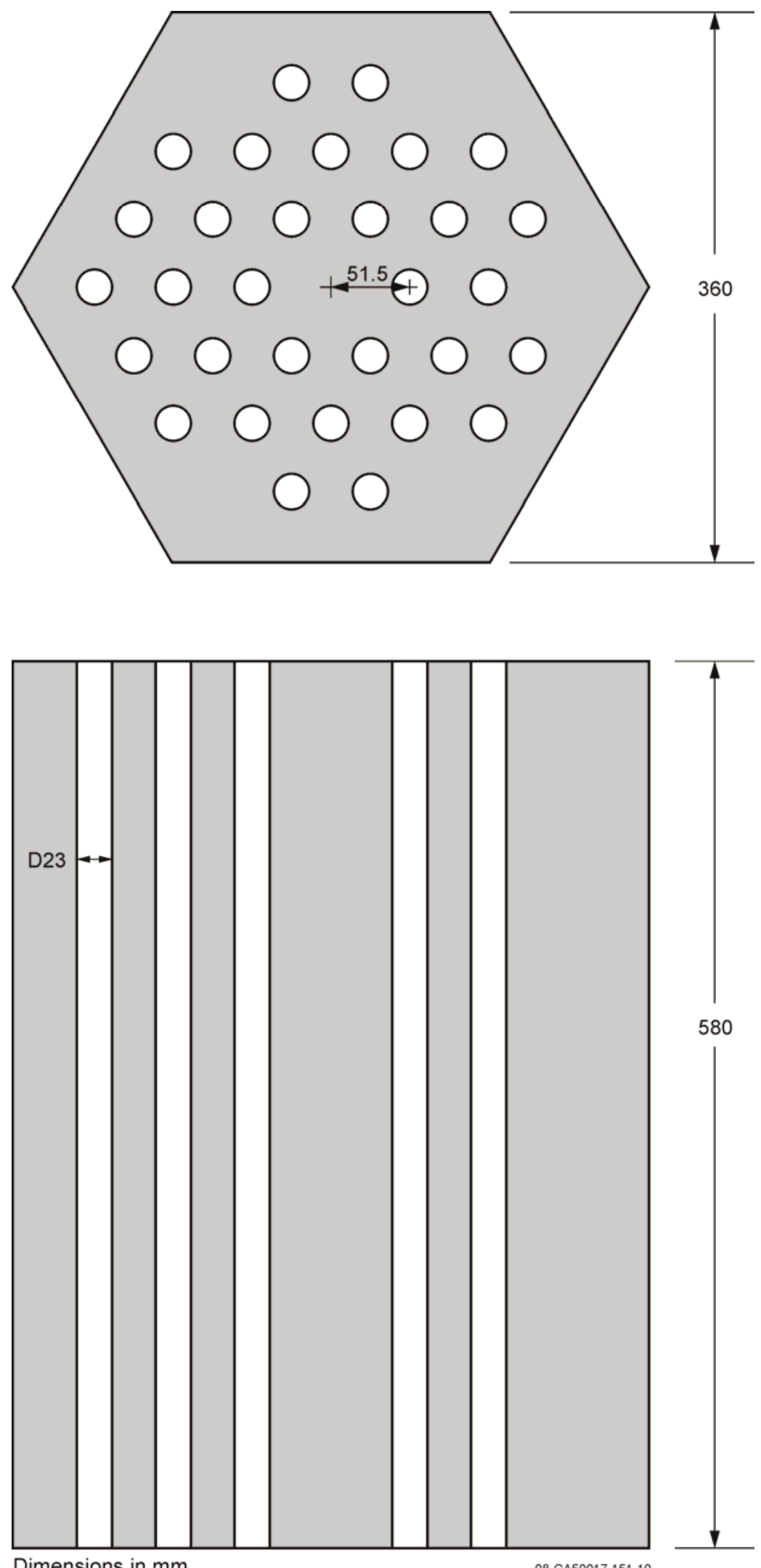

Figure 3.14. Replaceable Reflector Block for 31-Pin Fuel Assembly. Dxx represents the diameter in $\mathrm{xx}(\mathrm{mm})$. 
NEA/NSC/DOC(2006)1

Gas Cooled (Thermal) Reactor - GCR

HTTR-GCR-RESR-002

CRIT-REAC-RRATE

\subsubsection{Fuel Columns}

The fuel columns are separated into four zones (as shown in Figure 1.46 of HTTR-GCR-RESR-001). Each zone has a specified pattern of uranium enrichment. Each column contains two top replaceable reflector blocks (Figure 3.13 for Zones 1 and 2 or Figure 3.14 for Zones 3 and 4), five fuel blocks (Figure 3.5 for Zones 1 and 2 and Figure 3.6 for Zones 3 and 4), and two bottom replaceable reflector blocks (Figure 3.13 for Zones 1 and 2 or Figure 3.14 for Zones 3 and 4). The second and third fuel blocks from the top contain burnable poison pellets that are more enriched than the pellets in the other three positions. Figure 3.15 shows the enrichment of the uranium (wt.\%) in the TRISO fuel (upper left) and the natural boron content (wt.\%) in the burnable pellets (lower right).

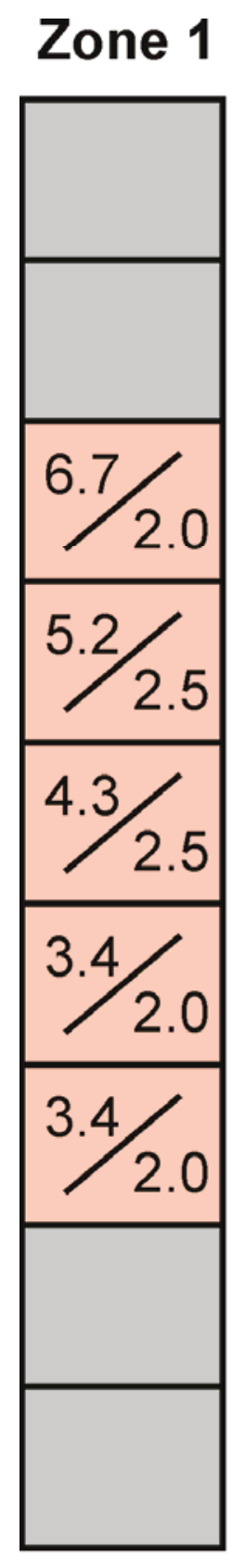

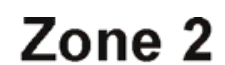

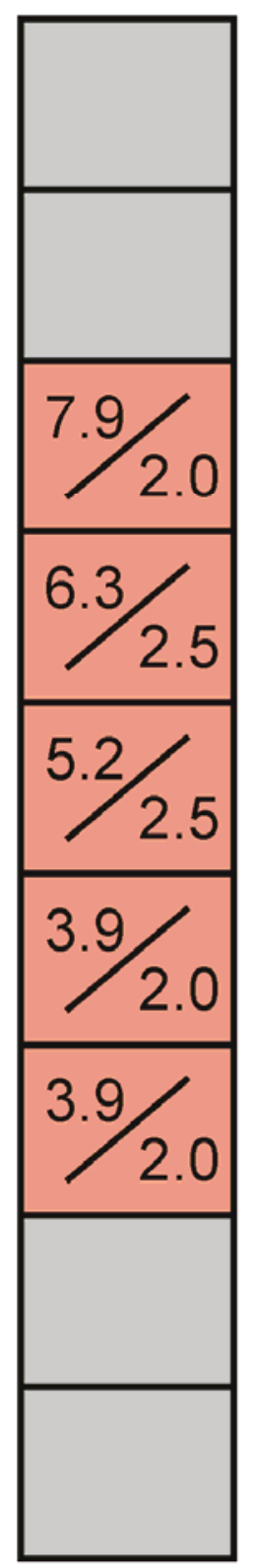

Zone 3

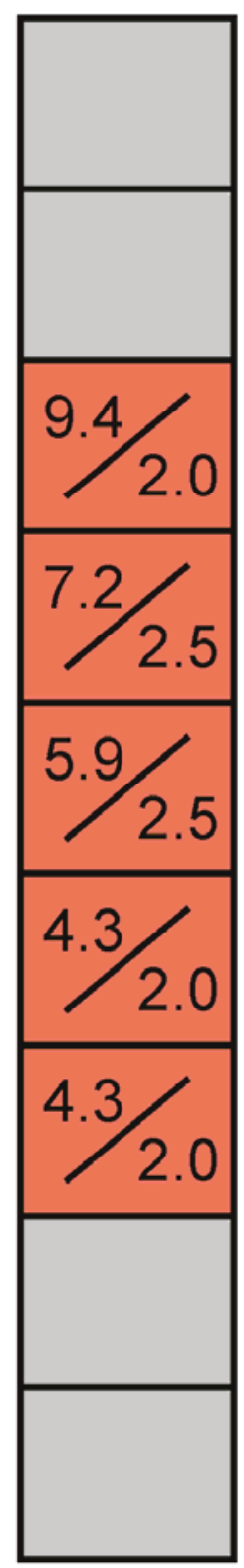

Figure 3.15. HTTR Fuel Zones.
Zone 4

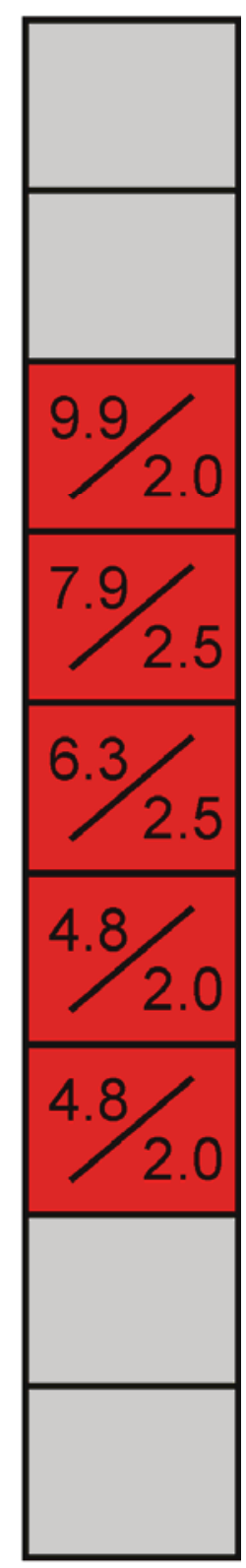

08-GA50017-167-1 


\subsubsection{Dummy Fuel Columns}

The dummy fuel columns are similar to the fuel columns shown in Figure 3.15 except that the fueled blocks are replaced with dummy fuel blocks. Each column therefore contains two top replaceable reflector blocks (Figures 3.13 or 3.14 if replacing Zones 1 and 2 or Zones 3 and 4, respectively), five dummy blocks (Figure 3.7), and two bottom replaceable reflector blocks (Figures 3.13 or 3.14). A dummy fuel column is shown in Figure 3.16.

\section{Dummy \\ Zone}

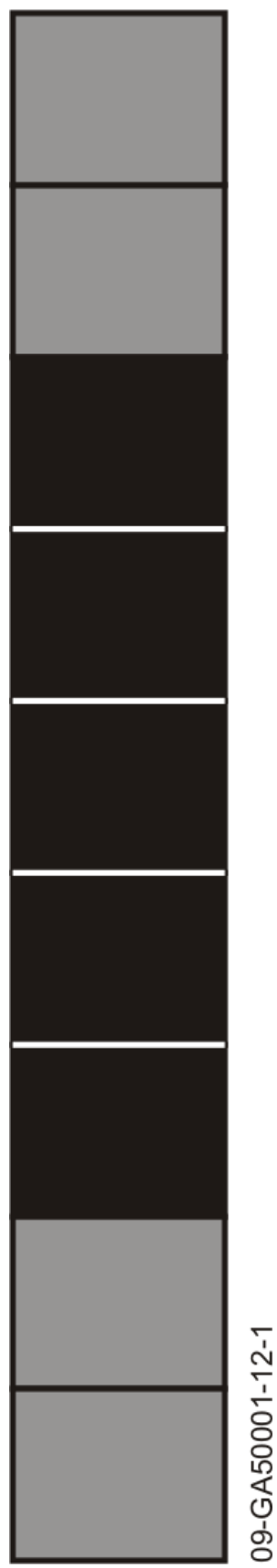

Figure 3.16. HTTR Dummy Fuel Zone. 
NEA/NSC/DOC(2006)1

Gas Cooled (Thermal) Reactor - GCR

HTTR-GCR-RESR-002

CRIT-REAC-RRATE

\subsubsection{Reactor Core Configuration}

Figures 3.17 through 3.20 are included to provide an understanding of the final configuration of the reactor. All of the annular core configurations have the same configuration and orientation as the fullyloaded core, but with dummy fuel columns in place of some of the fuel columns, as shown in Figures 3.21 through 3.24 .

The HTTR fully-loaded, 30-fuel-column, core configuration (HTTR-GCR-RESR-001) is shown in Figures 3.17, 3.18, 3.19. The first figure identifies the positions in the core for a given column type. The second figure provides the orientation of each column within its respective position in the core. The third figure shows the column identification number for each position in the core. Figure 3.20 shows a basic cross section of the HTTR fully-loaded core generated in MCNP.

The five configurations in this benchmark analysis use four core layouts, with the third core layout implementing two different control rod positions. Configuration 1 (Figure 3.21) has 19 fuel columns and 11 dummy fuel columns. Configuration 2 (Figure 3.22) has 21 fuel columns and 9 dummy fuel columns. Configurations 3 and 4 (Figure 3.23) have 24 fuel columns and 6 dummy fuel columns. Configuration 5 (Figure 3.24) has 27 fuel columns and 3 dummy fuel columns.

The permanent reflector surrounding the core has been circularized with a radius of $2125 \mathrm{~mm}$ and height of $5220 \mathrm{~mm}$. 
NEA/NSC/DOC(2006)1

Gas Cooled (Thermal) Reactor - GCR

HTTR-GCR-RESR-002

CRIT-REAC-RRATE

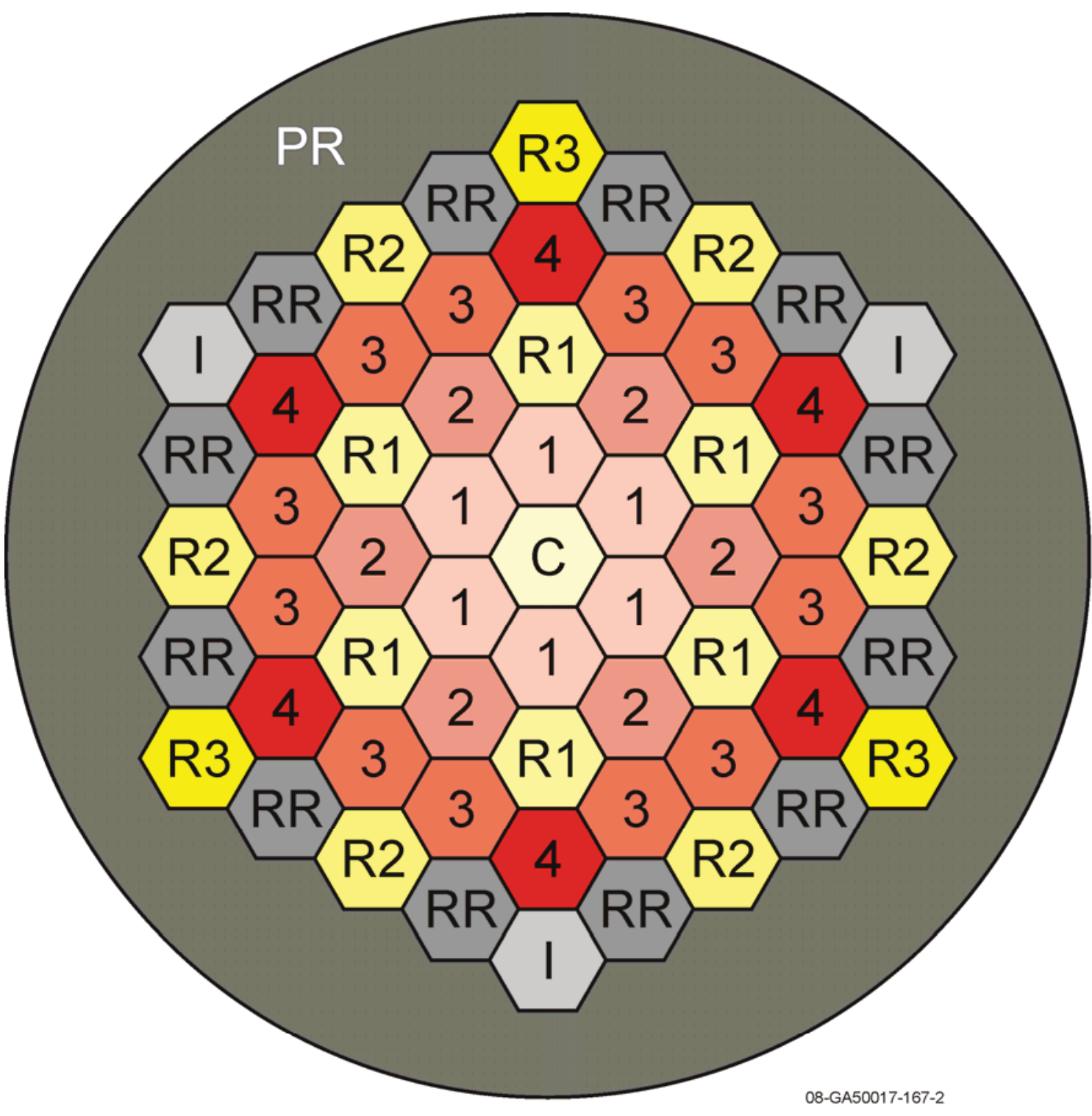

Fuel zones

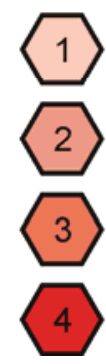

Control rods

C

Instrumentation

(1)

R1

Replaceable reflectors

RR

R2

Permanent reflector

Figure 3.17. HTTR Core Positions (Fully-Loaded, 30-Fuel-Column Core - No Dummy Fuel Columns). 
NEA/NSC/DOC(2006)1

Gas Cooled (Thermal) Reactor - GCR

HTTR-GCR-RESR-002

CRIT-REAC-RRATE

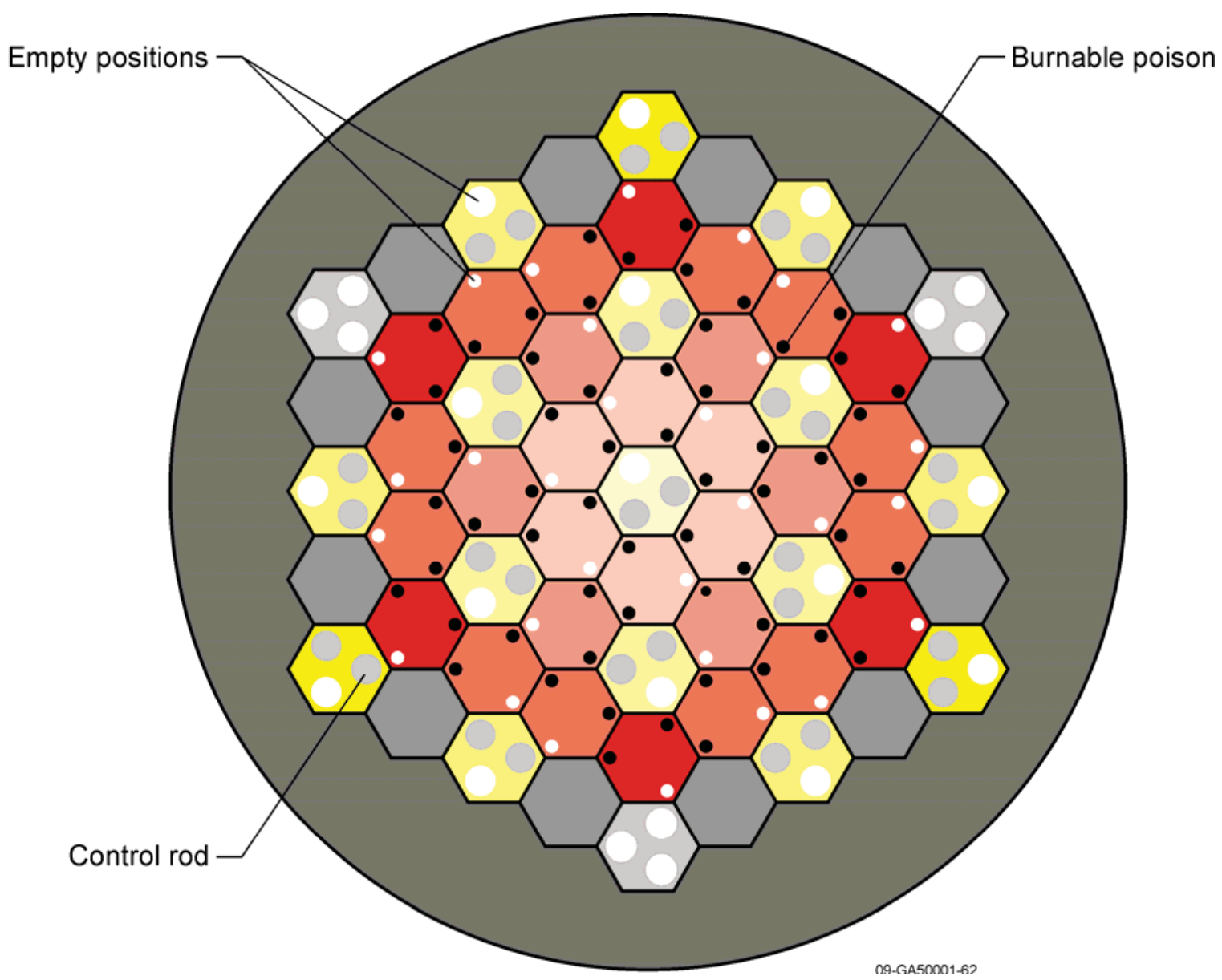

Figure 3.18. Fuel and Control Rod Column Orientations. 
NEA/NSC/DOC(2006)1

Gas Cooled (Thermal) Reactor - GCR

HTTR-GCR-RESR-002

CRIT-REAC-RRATE

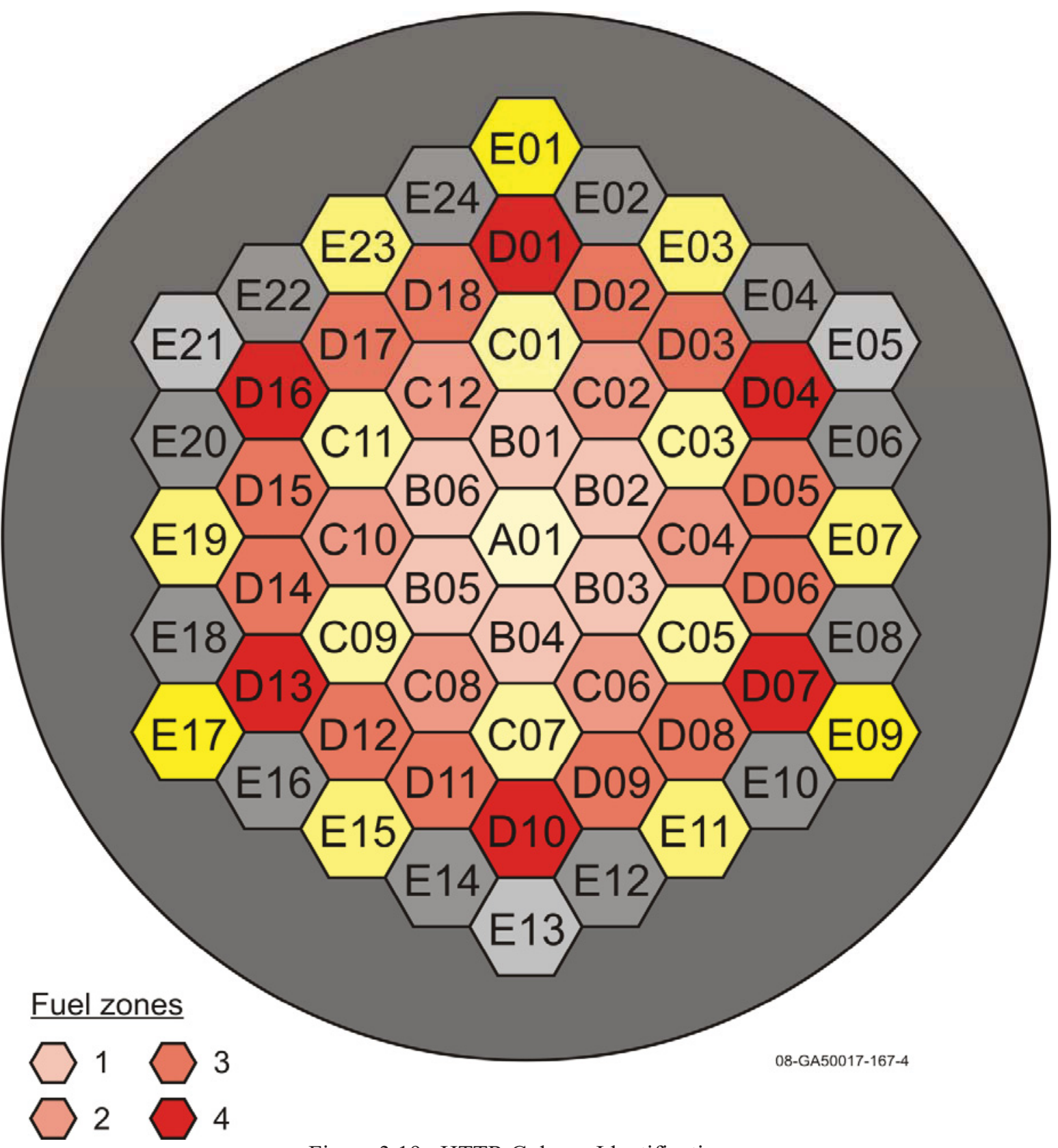

Figure 3.19. HTTR Column Identification. 
NEA/NSC/DOC(2006)1

Gas Cooled (Thermal) Reactor - GCR

\section{HTTR-GCR-RESR-002 \\ CRIT-REAC-RRATE}

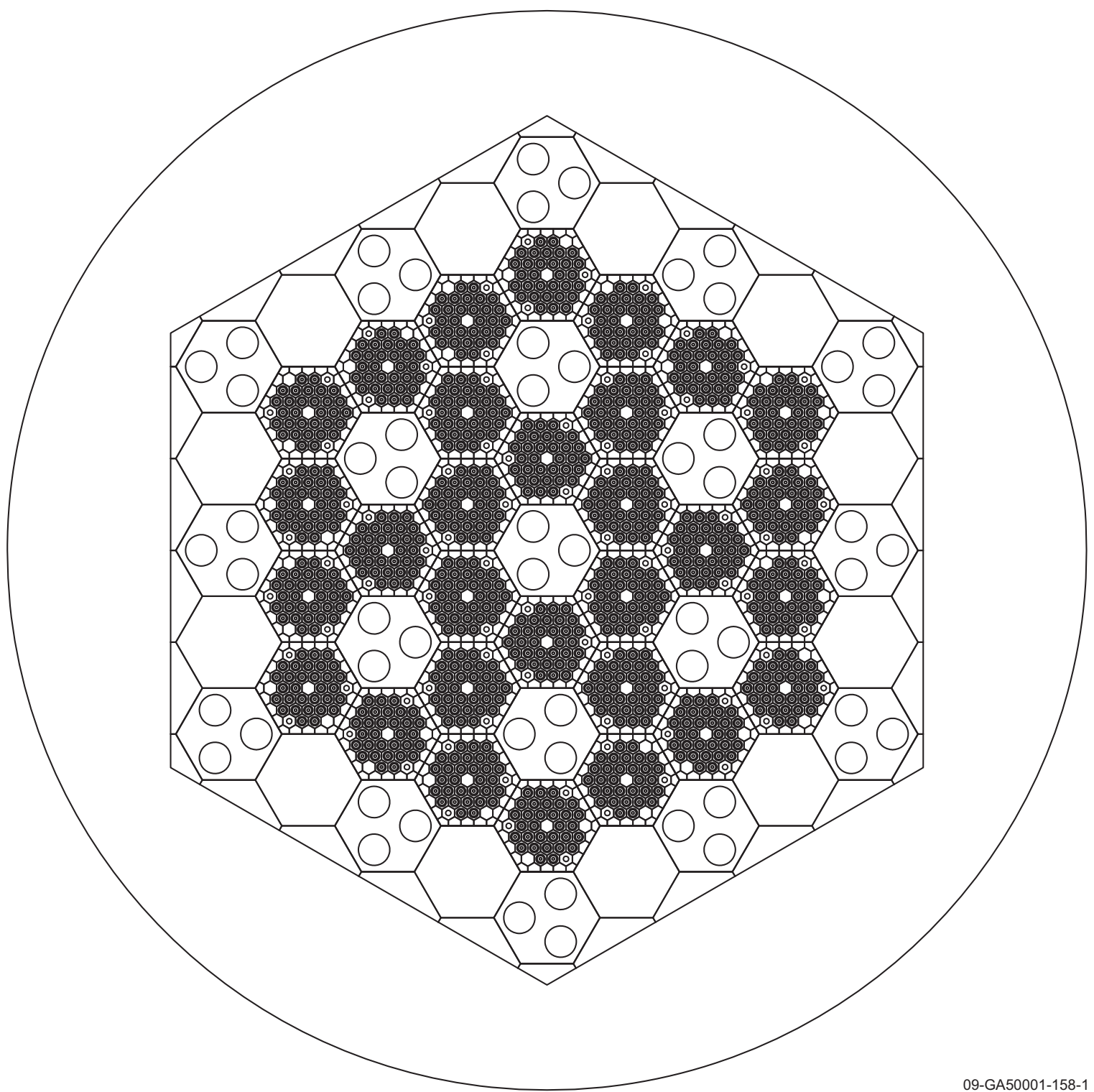

Figure 3.20. Cross Section of the HTTR Fully-Loaded, 30-Fuel-Column Core. 
NEA/NSC/DOC(2006)1

Gas Cooled (Thermal) Reactor - GCR

HTTR-GCR-RESR-002

CRIT-REAC-RRATE

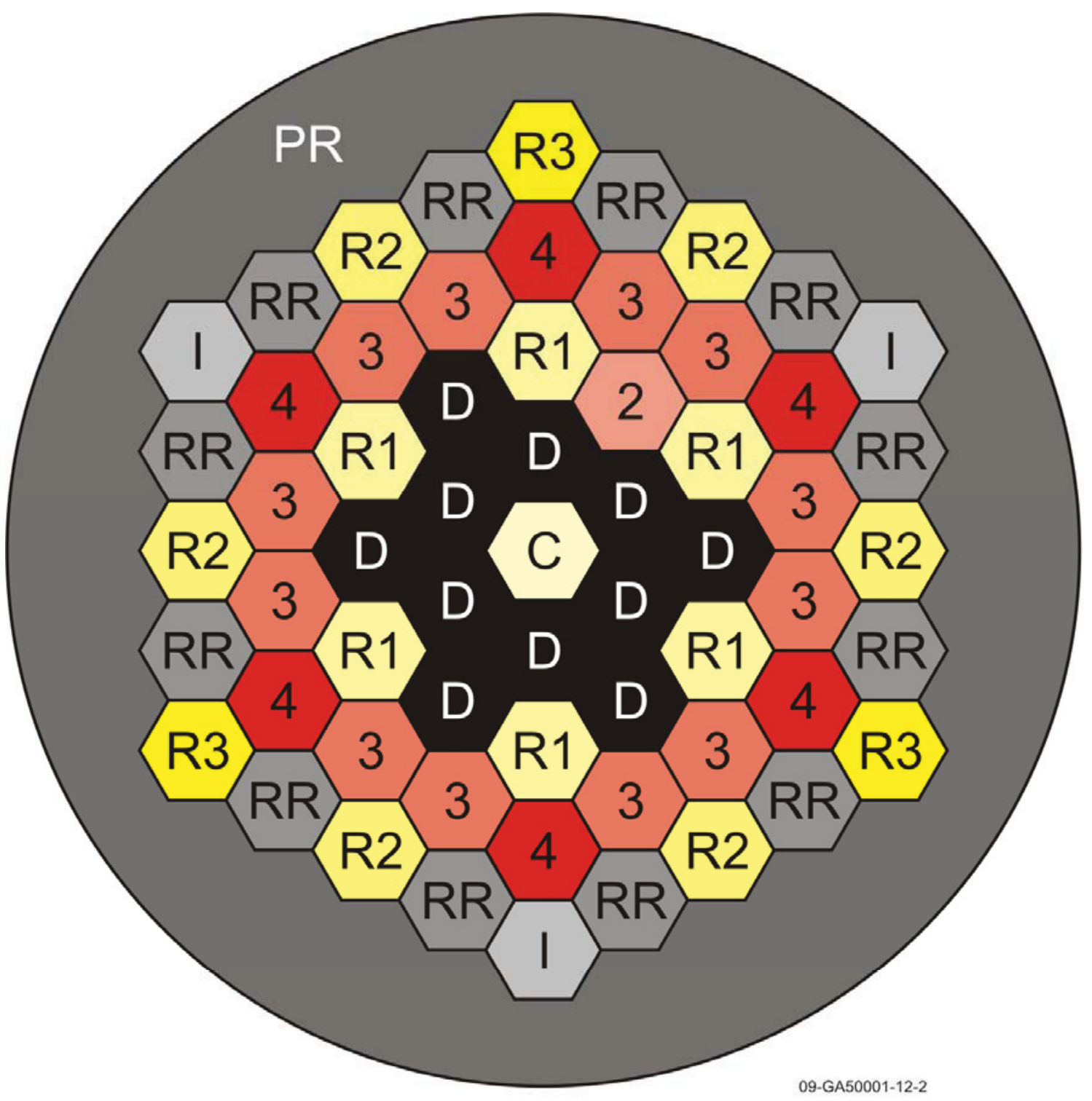

Fuel zones

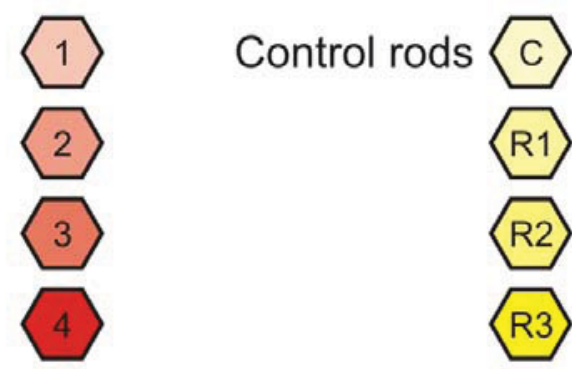

Dummy zone

Instrumentation

(1)

Replaceable reflectors

RR

Permanent reflector

Figure 3.21. HTTR Core Positions (Configuration 1, 19-Fuel-Columns). 
NEA/NSC/DOC(2006)1

Gas Cooled (Thermal) Reactor - GCR

HTTR-GCR-RESR-002

CRIT-REAC-RRATE

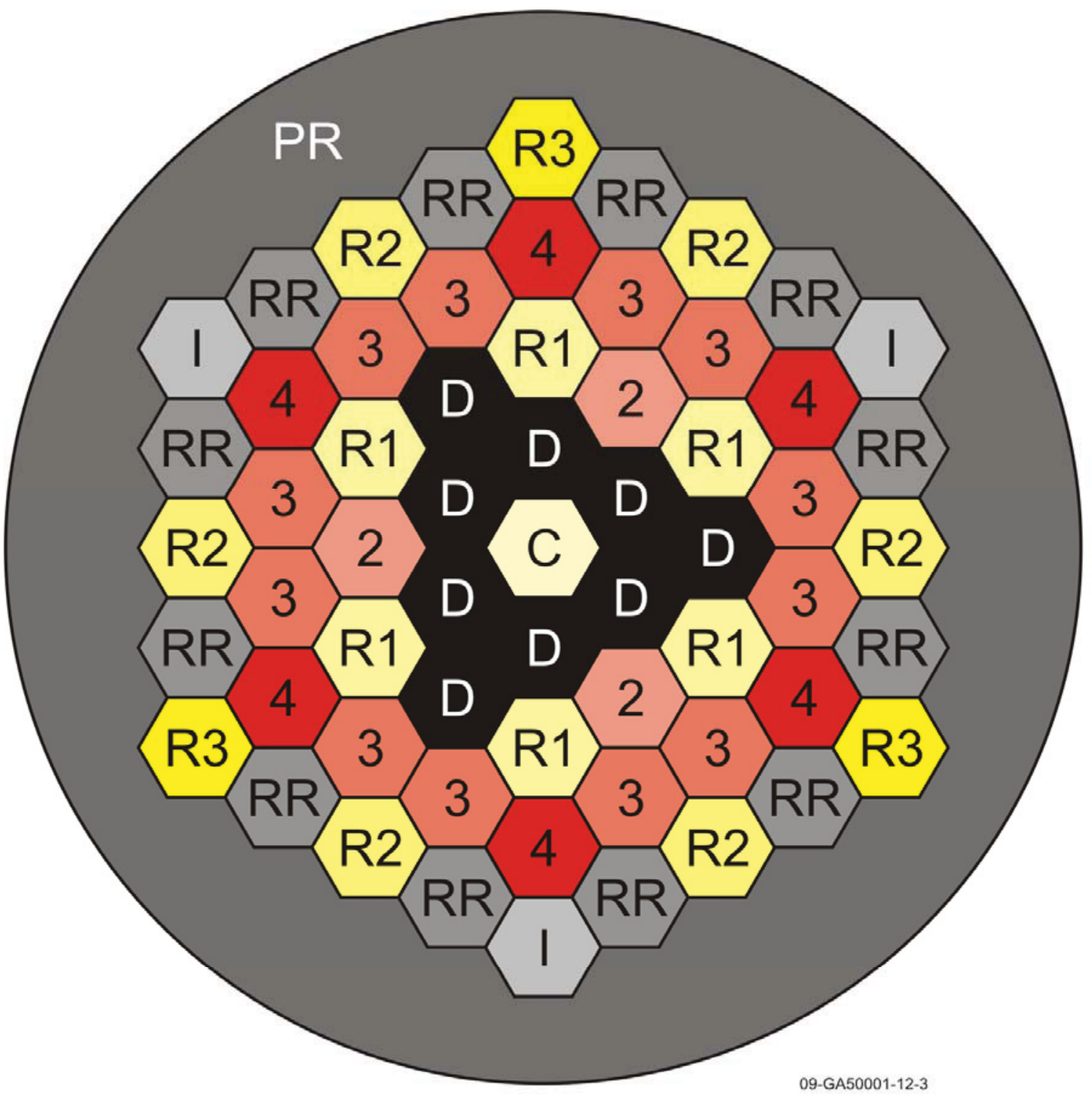

Fuel zones

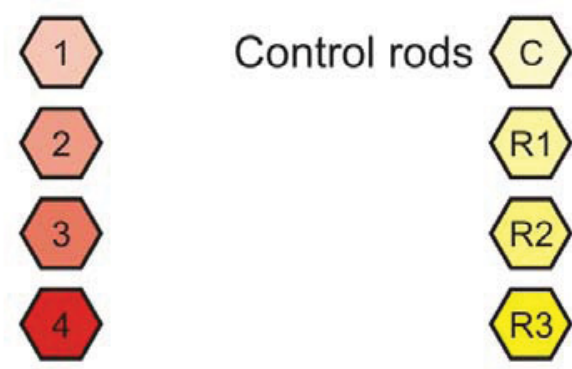

Dummy zone

Instrumentation

(1)

R2 Replaceable reflectors RR

R3 Permanent reflector

Figure 3.22. HTTR Core Positions (Configuration 2, 21-Fuel-Columns). 
NEA/NSC/DOC(2006)1

Gas Cooled (Thermal) Reactor - GCR

HTTR-GCR-RESR-002

CRIT-REAC-RRATE

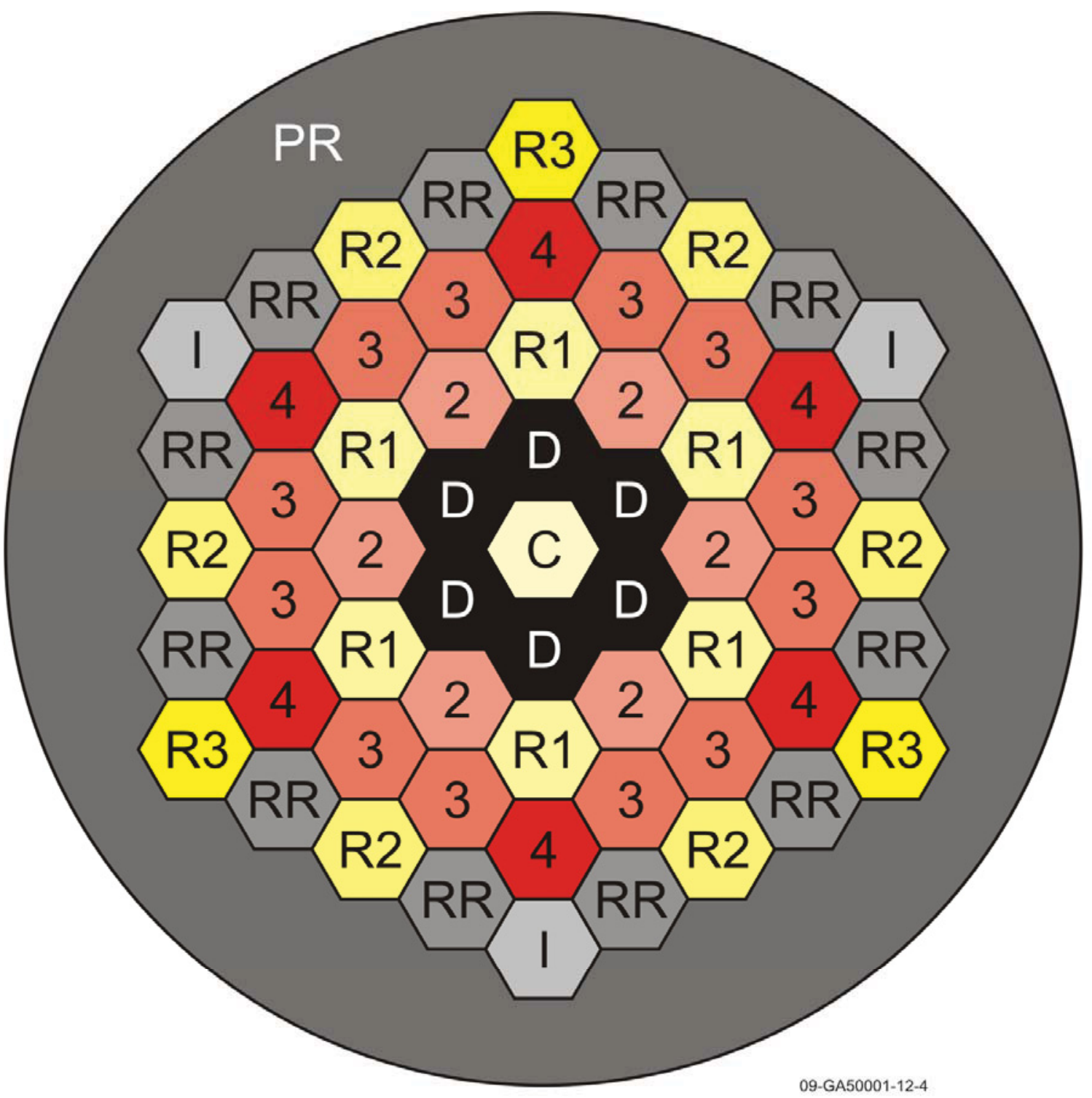

Fuel zones

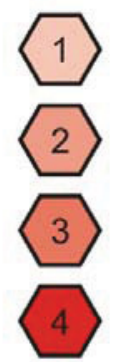

Control rods

C

Dummy zone

Instrumentation

(1)

R2

Replaceable reflectors

RR

R3

Permanent reflector

Figure 3.23. HTTR Core Positions (Configurations 3 and 4, 24-Fuel-Columns). 
NEA/NSC/DOC(2006)1

Gas Cooled (Thermal) Reactor - GCR

HTTR-GCR-RESR-002

CRIT-REAC-RRATE

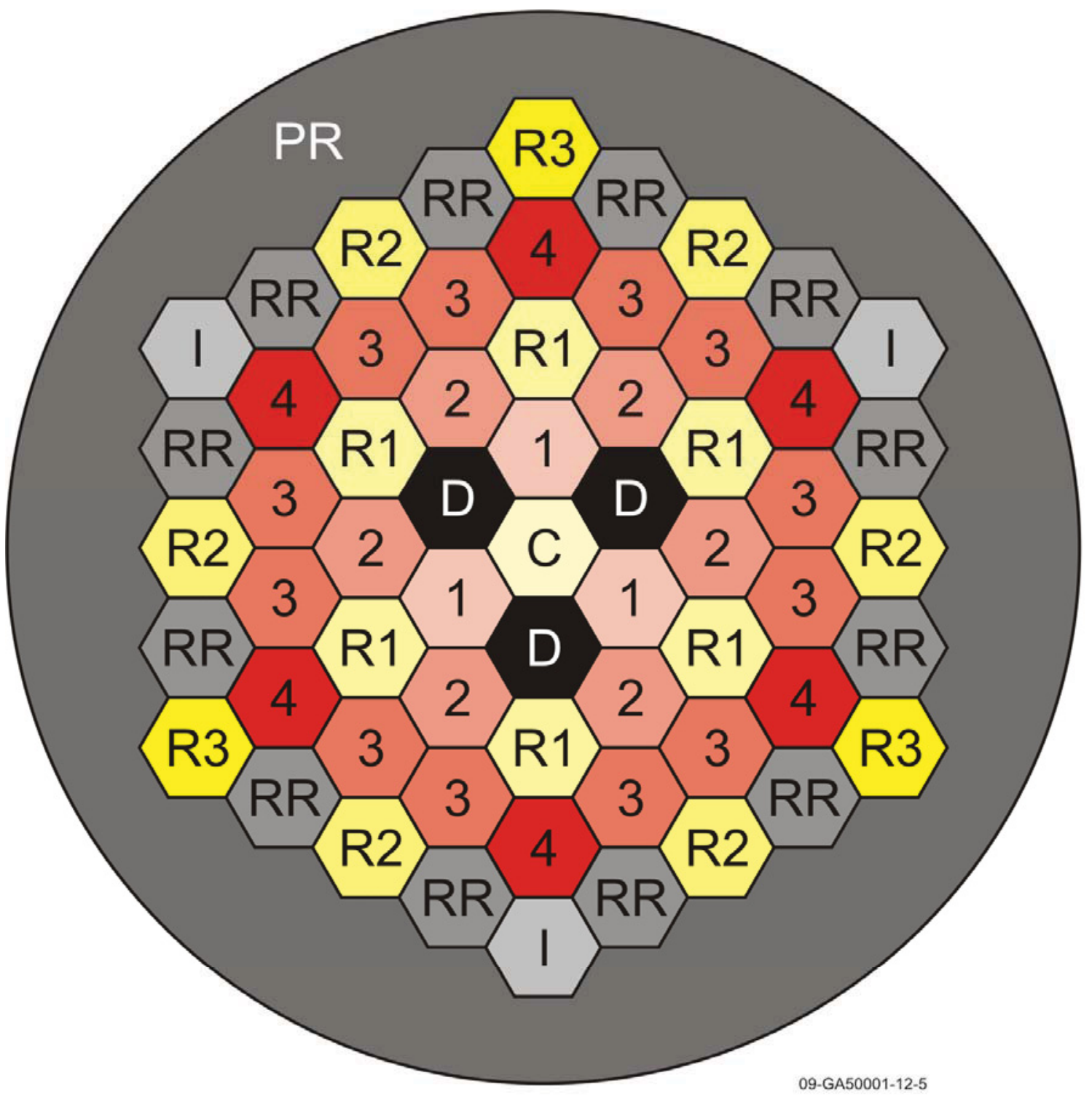

Fuel zones

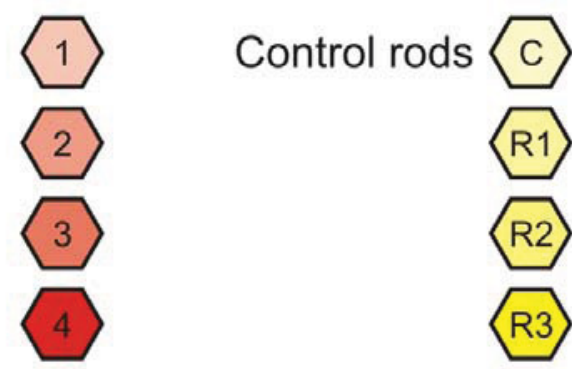

Dummy zone

Instrumentation

(1)

Replaceable reflectors

RR

Permanent reflector

Figure 3.24. HTTR Core Positions (Configuration 5, 27-Fuel-Columns). 
NEA/NSC/DOC(2006)1

\section{Gas Cooled (Thermal) Reactor - GCR \\ HTTR-GCR-RESR-002 \\ CRIT-REAC-RRATE}

\subsubsection{Critical Rod Positions}

The critical rod positions for configurations 1 through 5 are shown in Figures 3.25 through 3.29, respectively. Control rod positions are described with the zero position defined as level with the bottom place of the lowest fuel block (i.e. $1160 \mathrm{~mm}$ from the bottom of the core graphite, the lowest fuel block). These figures provide reference between the various column types in the core and the control rod positions; the dummy fuel column and fuel column are interchangeable in these figures, with no change in the dimensions.

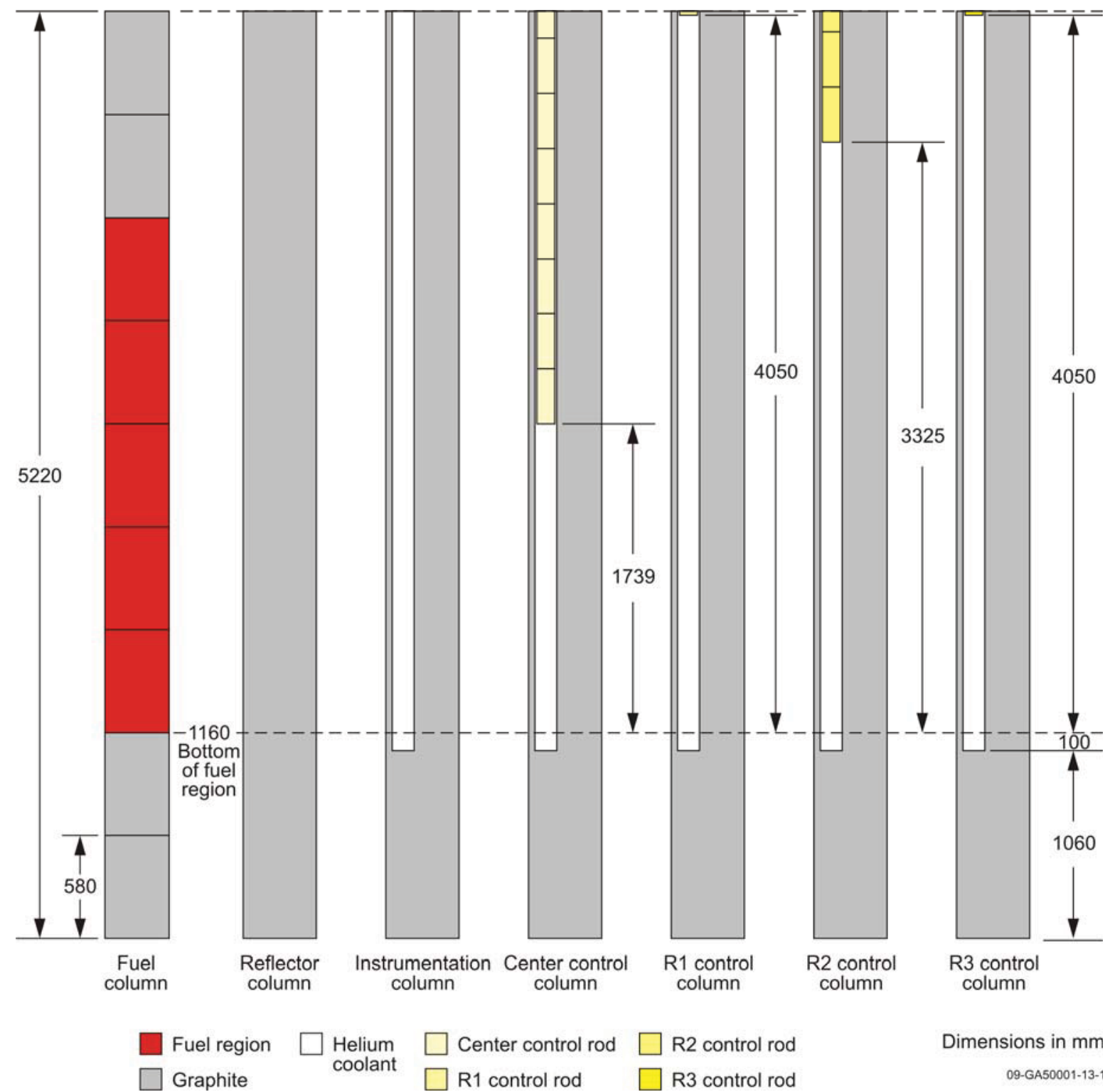

Figure 3.25. Axial Profile of Columns and Control Rod Positions (Configuration 1). 
NEA/NSC/DOC(2006)1

Gas Cooled (Thermal) Reactor - GCR

HTTR-GCR-RESR-002

CRIT-REAC-RRATE

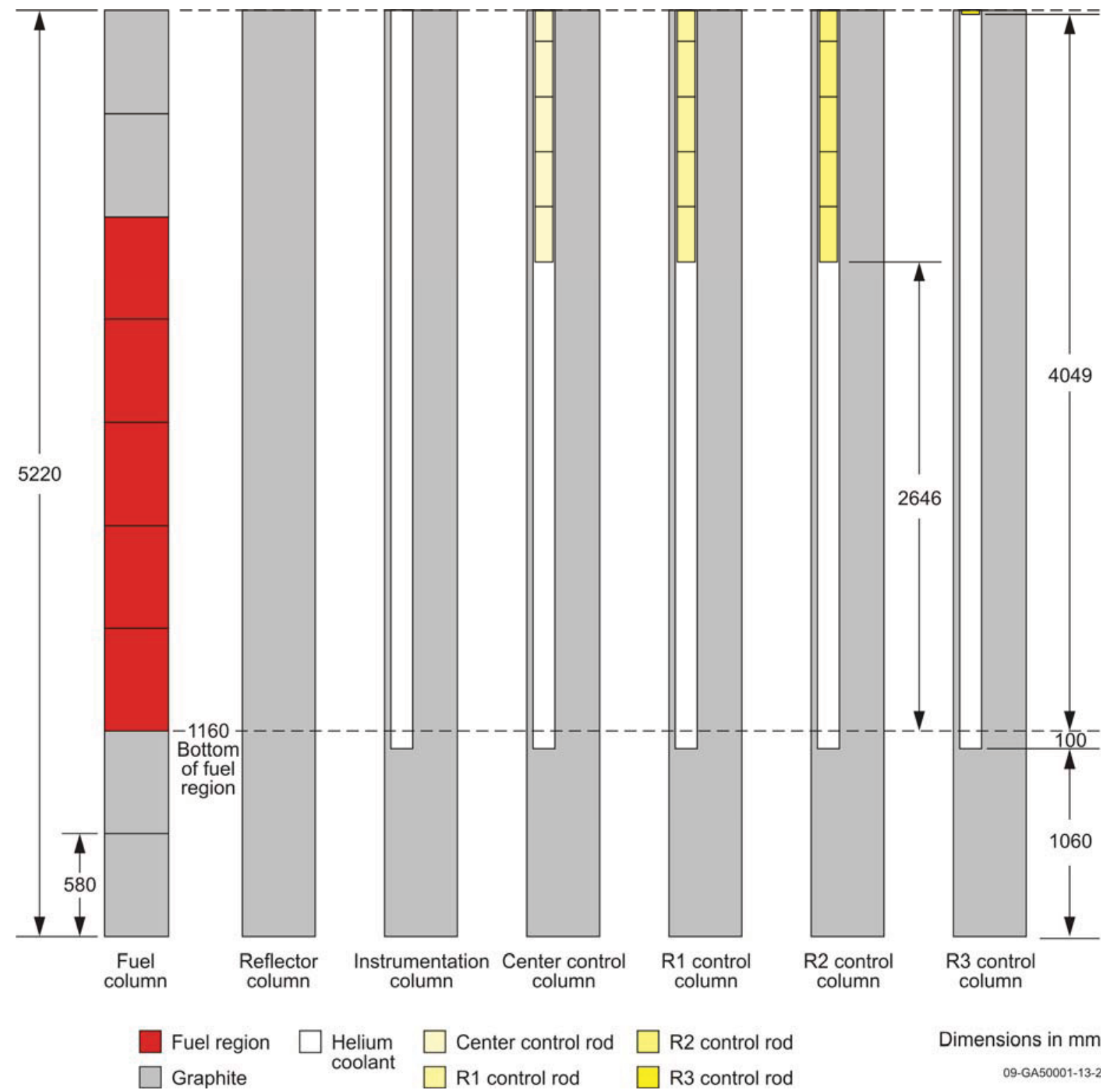

Figure 3.26. Axial Profile of Columns and Control Rod Positions (Configuration 2). 
NEA/NSC/DOC(2006)1

Gas Cooled (Thermal) Reactor - GCR

HTTR-GCR-RESR-002

CRIT-REAC-RRATE

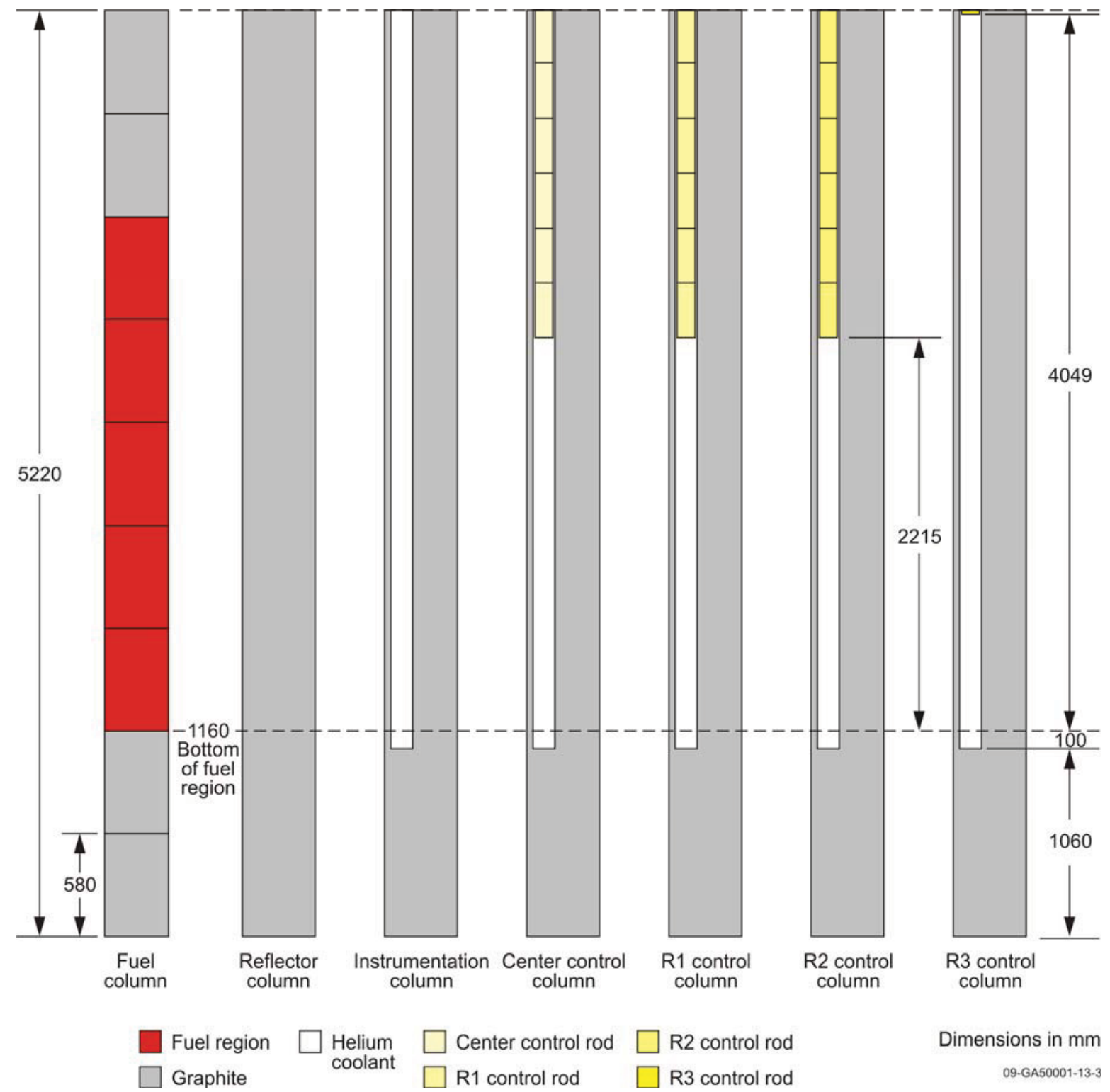

Figure 3.27. Axial Profile of Columns and Control Rod Positions (Configuration 3). 
NEA/NSC/DOC(2006)1

Gas Cooled (Thermal) Reactor - GCR

HTTR-GCR-RESR-002

CRIT-REAC-RRATE

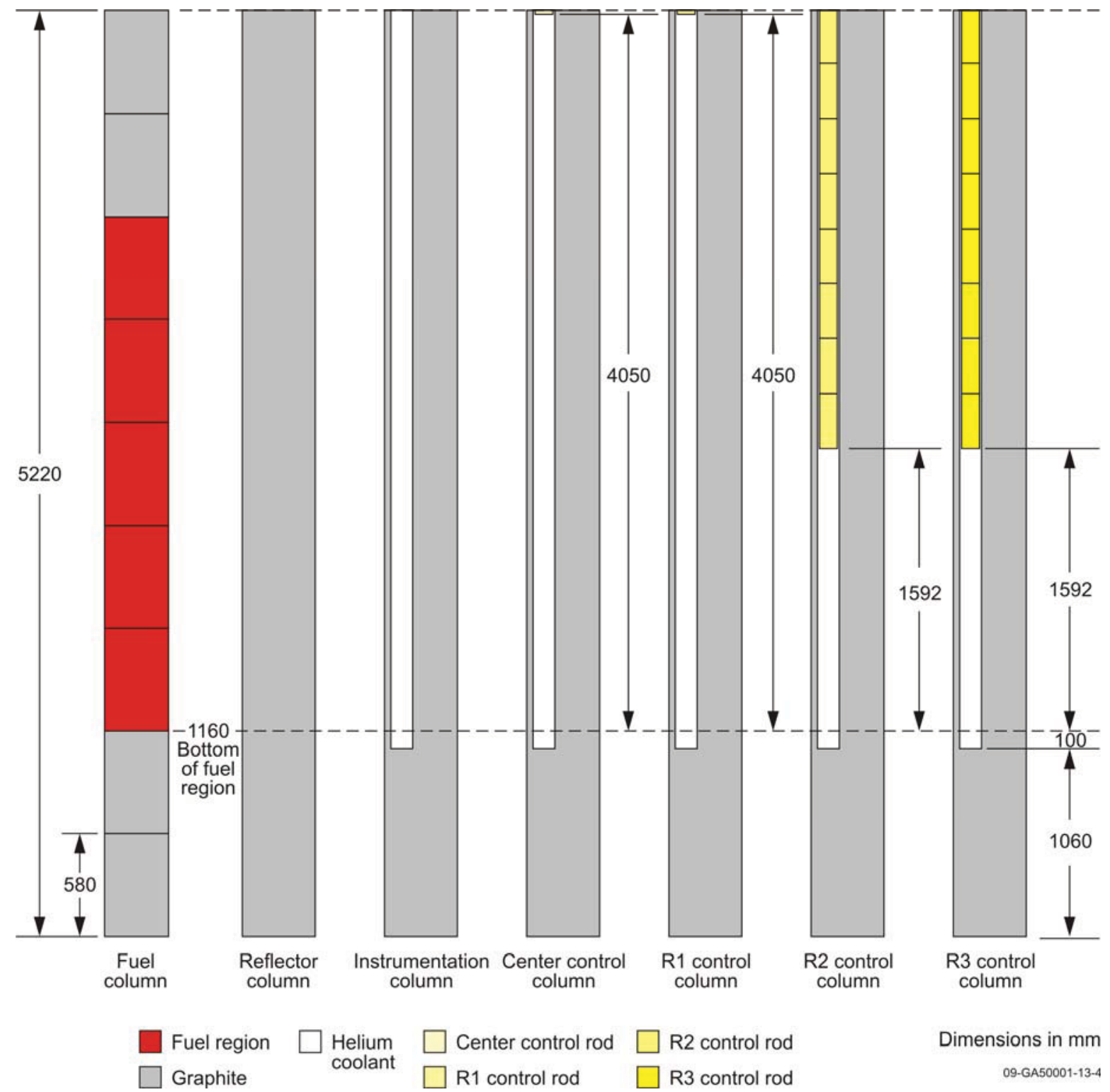

Figure 3.28. Axial Profile of Columns and Control Rod Positions (Configuration 4). 
NEA/NSC/DOC(2006)1

Gas Cooled (Thermal) Reactor - GCR

HTTR-GCR-RESR-002

CRIT-REAC-RRATE

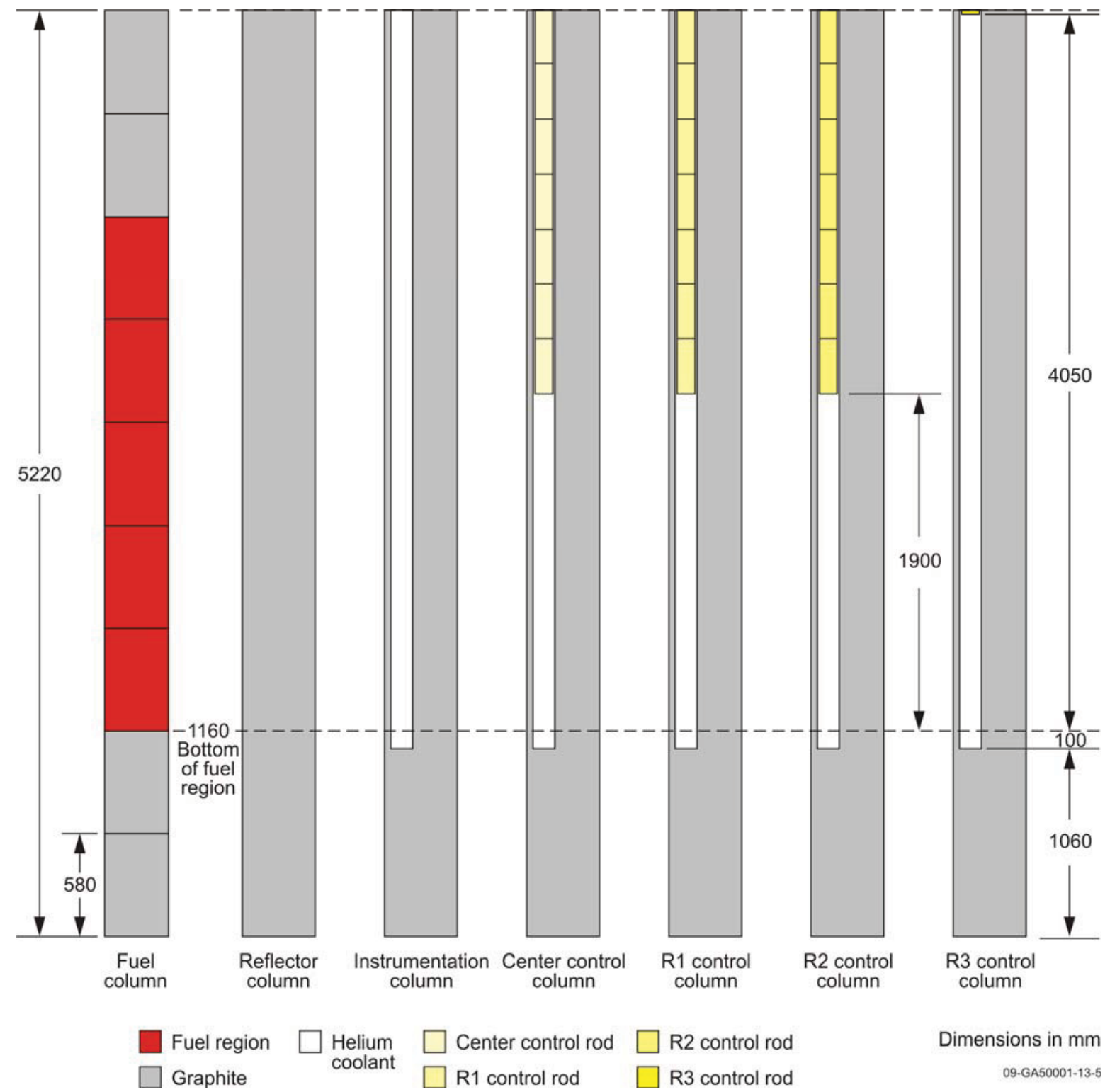

Figure 3.29. Axial Profile of Columns and Control Rod Positions (Configuration 5). 
NEA/NSC/DOC(2006)1

Gas Cooled (Thermal) Reactor - GCR

HTTR-GCR-RESR-002

CRIT-REAC-RRATE

\subsubsection{Material Data}

\subsubsection{Pin-in-Block Fuel}

\section{$\underline{\text { TRISO Particles }}$}

The mass density of the TRISO-coated $\mathrm{UO}_{2}$ fuel kernels is $10.40363 \mathrm{~g} / \mathrm{cm}^{3}$ (such that the total uranium mass per fuel rod is $188.58 \mathrm{~g}$ ) with an $\mathrm{O} / \mathrm{U}$ ratio of $\sim 2.00$ and an equivalent natural-boron impurity content of 0.00015 wt.\%. A summary of the atomic densities and compositions for the twelve enrichments found throughout the core are provided in Table 3.2.

Table 3.2. Atomic Densities (atoms/b-cm) of the $\mathrm{UO}_{2}$ Kernels for Varying Enrichments.

\begin{tabular}{||c|cccccc||}
\hline \hline Isotope & 3.40 wt. $\%$ & 3.90 wt. $\%$ & 4.30 wt. $\%$ & 4.80 wt. $\%$ & 5.20 wt. $\%$ & 5.90 wt. $\%$ \\
\hline \hline${ }^{10} \mathrm{~B}$ & $1.7299 \mathrm{E}-07$ & $1.7299 \mathrm{E}-07$ & $1.7299 \mathrm{E}-07$ & $1.7299 \mathrm{E}-07$ & $1.7299 \mathrm{E}-07$ & $1.7299 \mathrm{E}-07$ \\
$\mathrm{O}$ & $4.6404 \mathrm{E}-02$ & $4.6404 \mathrm{E}-02$ & $4.6404 \mathrm{E}-02$ & $4.6404 \mathrm{E}-02$ & $4.6404 \mathrm{E}-02$ & $4.6404 \mathrm{E}-02$ \\
${ }^{234} \mathrm{U}$ & $6.1026 \mathrm{E}-06$ & $7.0000 \mathrm{E}-06$ & $7.7180 \mathrm{E}-06$ & $8.6154 \mathrm{E}-06$ & $9.3334 \mathrm{E}-06$ & $1.0590 \mathrm{E}-05$ \\
${ }^{235} \mathrm{U}$ & $7.9888 \mathrm{E}-04$ & $9.1637 \mathrm{E}-04$ & $1.0104 \mathrm{E}-03$ & $1.1278 \mathrm{E}-03$ & $1.2218 \mathrm{E}-03$ & $1.3863 \mathrm{E}-03$ \\
${ }^{238} \mathrm{U}$ & $2.2405 \mathrm{E}-02$ & $2.2288 \mathrm{E}-02$ & $2.2195 \mathrm{E}-02$ & $2.2078 \mathrm{E}-02$ & $2.1984 \mathrm{E}-02$ & $2.1821 \mathrm{E}-02$ \\
\hline \hline Total & $6.9614 \mathrm{E}-02$ & $6.9616 \mathrm{E}-02$ & $6.9617 \mathrm{E}-02$ & $6.9618 \mathrm{E}-02$ & $6.9619 \mathrm{E}-02$ & $6.9622 \mathrm{E}-02$ \\
\hline \hline
\end{tabular}

Table 3.2 (cont'd.). Atomic Densities (atoms/b-cm) of the $\mathrm{UO}_{2}$ Kernels for Varying Enrichments.

\begin{tabular}{||c|cccccc||}
\hline \hline Isotope & 6.30 wt. $\%$ & 6.70 wt. $\%$ & 7.20 wt. $\%$ & 7.90 wt. $\%$ & 9.40 wt. $\%$ & 9.90 wt. $\%$ \\
\hline \hline${ }^{10} \mathrm{~B}$ & $1.7299 \mathrm{E}-07$ & $1.7299 \mathrm{E}-07$ & $1.7299 \mathrm{E}-07$ & $1.7299 \mathrm{E}-07$ & $1.7299 \mathrm{E}-07$ & $1.7299 \mathrm{E}-07$ \\
$\mathrm{O}$ & $4.6404 \mathrm{E}-02$ & $4.6404 \mathrm{E}-02$ & $4.6404 \mathrm{E}-02$ & $4.6404 \mathrm{E}-02$ & $4.6404 \mathrm{E}-02$ & $4.6404 \mathrm{E}-02$ \\
${ }^{234} \mathrm{U}$ & $1.1308 \mathrm{E}-05$ & $1.2026 \mathrm{E}-05$ & $1.2923 \mathrm{E}-05$ & $1.4180 \mathrm{E}-05$ & $1.6872 \mathrm{E}-05$ & $1.7769 \mathrm{E}-05$ \\
${ }^{235} \mathrm{U}$ & $1.4803 \mathrm{E}-03$ & $1.5743 \mathrm{E}-03$ & $1.6918 \mathrm{E}-03$ & $1.8562 \mathrm{E}-03$ & $2.2087 \mathrm{E}-03$ & $2.3262 \mathrm{E}-03$ \\
${ }^{238} \mathrm{U}$ & $2.1727 \mathrm{E}-02$ & $2.1634 \mathrm{E}-02$ & $2.1517 \mathrm{E}-02$ & $2.1353 \mathrm{E}-02$ & $2.1002 \mathrm{E}-02$ & $2.0886 \mathrm{E}-02$ \\
\hline \hline Total & $6.9623 \mathrm{E}-02$ & $6.9624 \mathrm{E}-02$ & $6.9625 \mathrm{E}-02$ & $6.9628 \mathrm{E}-02$ & $6.9632 \mathrm{E}-02$ & $6.9634 \mathrm{E}-02$ \\
\hline \hline
\end{tabular}

The material properties of the TRISO layers and graphite overcoat are provided in Table 3.3.

Table 3.3. Material Properties of the TRISO Coatings and Graphite Overcoat.

\begin{tabular}{|c|c|c|c|c|c|}
\hline Property & Buffer & IPyC & $\mathrm{SiC}$ & OPyC & Overcoat \\
\hline Mass Density $\left(\mathrm{g} / \mathrm{cm}^{3}\right)$ & 1.1 & 1.85 & 3.2 & 1.85 & 1.7 \\
\hline B-nat Impurity (wppm) & 1.5 & 1.5 & 1.5 & 1.5 & 1.5 \\
\hline $\begin{array}{l}\text { Atomic Density } \\
\text { (atoms } / \mathrm{b}-\mathrm{cm})\end{array}$ & $5.5153 \mathrm{E}-02$ & $9.2758 \mathrm{E}-02$ & $9.6122 \mathrm{E}-02$ & $9.2758 \mathrm{E}-02$ & 8.5237E-02 \\
\hline${ }^{10} \mathrm{~B}$ & $1.8290 \mathrm{E}-08$ & 3.0761E-08 & $5.3208 \mathrm{E}-08$ & $3.0761 \mathrm{E}-08$ & $2.8267 \mathrm{E}-08$ \\
\hline C-nat & $5.5153 \mathrm{E}-02$ & $9.2758 \mathrm{E}-02$ & $4.8061 \mathrm{E}-02$ & $9.2758 \mathrm{E}-02$ & $8.5237 \mathrm{E}-02$ \\
\hline $\mathrm{Si}$ & -- & -- & $4.8061 \mathrm{E}-02$ & -- & -- \\
\hline
\end{tabular}

Revision: 0 
NEA/NSC/DOC(2006)1

Gas Cooled (Thermal) Reactor - GCR

HTTR-GCR-RESR-002

CRIT-REAC-RRATE

\section{Compacts}

A key parameter is that the total fuel mass of a single fuel rod (14 stacked compacts) is approximately $188.58 \mathrm{~g}$.

The mass density of the fuel compact graphite matrix is $1.7 \mathrm{~g} / \mathrm{cm}^{3}$ with an equivalent natural-boron impurity content of $0.000082 \mathrm{wt} . \%$. The atomic density and composition of the compact matrix is shown in Table 3.4.

Table 3.4. Atomic Densities of the Fuel Compact Graphite Matrix.

\begin{tabular}{||c|c||}
\hline Isotope & Atoms/b-cm \\
\hline \hline${ }^{10} \mathrm{~B}$ & $1.5452 \mathrm{E}-08$ \\
C-nat & $8.5237 \mathrm{E}-02$ \\
\hline \hline Total & $8.5237 \mathrm{E}-02$ \\
\hline
\end{tabular}

\section{Fuel Element}

The IG-110 graphite sleeve and end caps for the fuel pins have a mass density of $1.77 \mathrm{~g} / \mathrm{cm}^{3}$ and an equivalent natural-boron content of $0.000037 \mathrm{wt} . \%$. The atomic density and composition of the graphite used in the fuel element is shown in Table 3.5.

Table 3.5. Atomic Densities of the Graphite Fuel Sleeve.

\begin{tabular}{||c|c||}
\hline Isotope & Atoms/b-cm \\
\hline \hline${ }^{10} \mathrm{~B}$ & $7.2596 \mathrm{E}-09$ \\
C-nat & $8.8747 \mathrm{E}-02$ \\
\hline \hline Total & $8.8747 \mathrm{E}-02$ \\
\hline
\end{tabular}

\subsubsection{Burnable Poisons}

The burnable poison pellets have a mass density of $1.80 \mathrm{~g} / \mathrm{cm}^{3}$; a summary of the atomic densities and compositions for the two natural-boron concentrations employed in the core are provided in Table 3.6. The mass density of the graphite disks used to separate the burnable poison pellets is $1.77 \mathrm{~g} / \mathrm{cm}^{3}$ with an equivalent natural-boron content of $0.000037 \mathrm{wt} . \%$. The atomic density and composition of the graphite disks is also found in Table 3.6 
NEA/NSC/DOC(2006)1

Gas Cooled (Thermal) Reactor - GCR

HTTR-GCR-RESR-002

CRIT-REAC-RRATE

Table 3.6. Atomic Densities (atoms $/ \mathrm{b}-\mathrm{cm}$ ) of the Burnable Poison Pellets and Graphite Disks.

\begin{tabular}{||c|ccc||}
\hline Isotope & $2.00 \mathrm{wt} . \%$ & $2.50 \mathrm{wt} . \%$ & Disks \\
\hline \hline${ }^{10} \mathrm{~B}$ & $3.9906 \mathrm{E}-04$ & $4.9882 \mathrm{E}-04$ & $7.2596 \mathrm{E}-09$ \\
${ }^{11} \mathrm{~B}$ & $1.6063 \mathrm{E}-03$ & $2.0078 \mathrm{E}-03$ & -- \\
C-nat & $8.8446 \mathrm{E}-02$ & $8.7995 \mathrm{E}-02$ & $8.8747 \mathrm{E}-02$ \\
\hline \hline Total & $9.0451 \mathrm{E}-02$ & $9.0501 \mathrm{E}-02$ & $8.8747 \mathrm{E}-02$ \\
\hline
\end{tabular}

\subsubsection{Fuel Blocks}

The IG-110 graphite fuel blocks have a mass density of $1.7512 \mathrm{~g} / \mathrm{cm}^{3}\left(1.76 \mathrm{~g} / \mathrm{cm}^{3}\right.$ base density decreased by a calculated $0.5 \%$ void fraction) and an equivalent natural-boron content of 0.000059 wt.\%. The atomic density and composition of the graphite fuel blocks is shown in Table 3.7.

Table 3.7. Atomic Densities of the Graphite Fuel Blocks.

\begin{tabular}{||c|c||}
\hline Isotope & Atoms/b-cm \\
\hline \hline${ }^{10} \mathrm{~B}$ & $1.1453 \mathrm{E}-08$ \\
C-nat & $8.7804 \mathrm{E}-02$ \\
\hline \hline Total & $8.7804 \mathrm{E}-02$ \\
\hline
\end{tabular}

\subsubsection{Dummy Blocks}

The IG-11 graphite fuel blocks have a mass density of $1.7413 \mathrm{~g} / \mathrm{cm}^{3}\left(1.75 \mathrm{~g} / \mathrm{cm}^{3}\right.$ base density decreased by a calculated $0.5 \%$ void fraction) and an equivalent natural-boron content of $0.00031 \mathrm{wt} . \%$. The atomic density and composition of the graphite dummy blocks is shown in Table 3.8.

Table 3.8. Atomic Densities of the Graphite Dummy Blocks.

\begin{tabular}{|c|c||}
\hline Isotope & Atoms $/ \mathrm{b}-\mathrm{cm}$ \\
\hline \hline${ }^{10} \mathrm{~B}$ & $5.9835 \mathrm{E}-08$ \\
C-nat & $8.7305 \mathrm{E}-02$ \\
\hline \hline Total & $8.7305 \mathrm{E}-02$ \\
\hline
\end{tabular}

\subsubsection{Control Rod System}

\section{Control Rods}

The absorber compacts have a mass density of $1.9 \mathrm{~g} / \mathrm{cm}^{3}$ and have a composition and atomic density as described in Table 3.9. 
NEA/NSC/DOC(2006)1

Gas Cooled (Thermal) Reactor - GCR

HTTR-GCR-RESR-002

CRIT-REAC-RRATE

Table 3.9. Atomic Densities of the Absorber Compacts.

\begin{tabular}{||c|c||}
\hline Isotope & Atoms $/ \mathrm{b}-\mathrm{cm}$ \\
\hline \hline${ }^{10} \mathrm{~B}$ & $6.3184 \mathrm{E}-03$ \\
${ }^{11} \mathrm{~B}$ & $2.5432 \mathrm{E}-02$ \\
C-nat & $6.6685 \mathrm{E}-02$ \\
\hline \hline Total & $9.8436 \mathrm{E}-02$ \\
\hline
\end{tabular}

The Alloy $800 \mathrm{H}$ cladding of the control rods has a mass density of $8.03 \mathrm{~g} / \mathrm{cm}^{3}$ with a composition and atomic density as shown in Table 3.10.

Table 3.10. Atomic Densities of the Alloy $800 \mathrm{H}$ Clad.

\begin{tabular}{||c|c||}
\hline Isotope & Atoms/b-cm \\
\hline \hline C-nat & $3.2210 \mathrm{E}-04$ \\
$\mathrm{Al}$ & $6.7209 \mathrm{E}-04$ \\
$\mathrm{Si}$ & $6.0263 \mathrm{E}-04$ \\
$\mathrm{P}$ & $3.1225 \mathrm{E}-05$ \\
$\mathrm{~S}$ & $1.5081 \mathrm{E}-05$ \\
$\mathrm{Ti}$ & $3.7884 \mathrm{E}-04$ \\
$\mathrm{Cr}$ & $1.9530 \mathrm{E}-02$ \\
$\mathrm{Mn}$ & $8.8022 \mathrm{E}-04$ \\
$\mathrm{Fe}$ & $3.8092 \mathrm{E}-02$ \\
$\mathrm{Ni}$ & $2.6777 \mathrm{E}-02$ \\
$\mathrm{Cu}$ & $2.2830 \mathrm{E}-04$ \\
\hline \hline $\mathrm{Total}$ & $8.7530 \mathrm{E}-02$ \\
\hline
\end{tabular}

\section{Control Rod Columns}

The IG-110 graphite fuel columns are modeled with the same physical properties as the fuel blocks in Section 3.1.3.3 and Table 3.7.

\subsubsection{Instrumentation}

\section{Instrumentation Components}

Insufficient information was available to adequately model instrumentation in the HTTR. An approximate bias with uncertainty was determined applied to the benchmark model (see Sections 2.1.2.6 and 3.1.1.1). 
NEA/NSC/DOC(2006)1

\section{Gas Cooled (Thermal) Reactor - GCR \\ HTTR-GCR-RESR-002 \\ CRIT-REAC-RRATE}

\section{Instrumentation Columns}

The IG-110 graphite instrumentation columns are modeled with the same physical properties as the fuel blocks in Section 3.1.3.3 and Table 3.7.

\subsubsection{Replaceable Reflector Columns}

The IG-110 replaceable reflector columns are modeled with the same physical properties as the fuel blocks in Section 3.1.3.3 and Table 3.7.

\subsubsection{Replaceable Reflectors Blocks in Fuel Columns}

The IG-110 replaceable reflector blocks are modeled with the same physical properties as the fuel blocks in Section 3.1.3.3 and Table 3.7.

\subsubsection{Permanent Reflector}

The PGX graphite permanent reflector has a mass density of $1.71789 \mathrm{~g} / \mathrm{cm}^{3}\left(1.76 \mathrm{~g} / \mathrm{cm}^{3}\right.$ base density decreased by a provided $0.7 \%$ void fraction) and an equivalent natural-boron content of $0.000191 \mathrm{wt} . \%$. The atomic density and composition of the permanent reflector is shown in Table 3.11.

Table 3.11. Atomic Densities of the Permanent Reflector.

\begin{tabular}{||c|c||}
\hline Isotope & Atoms/b-cm \\
\hline \hline${ }^{10} \mathrm{~B}$ & $3.6372 \mathrm{E}-08$ \\
C-nat & $8.6134 \mathrm{E}-02$ \\
\hline \hline Total & $8.6134 \mathrm{E}-02$ \\
\hline
\end{tabular}

\subsubsection{Helium Coolant}

The helium coolant has an atomic density of $2.4616 \mathrm{E}-05$ atoms $/ \mathrm{b}-\mathrm{cm}$ (mass density of $1.6361 \times 10^{-4}$ $\mathrm{g} / \mathrm{cm}^{3}$ ). No impurities are modeled in the coolant.

$$
\begin{gathered}
\frac{n}{V}=\frac{P \cdot N_{A}}{R \cdot T}=\frac{(1 \mathrm{~atm}) \cdot\left(0.60221 \frac{\mathrm{atoms} \cdot \mathrm{cm}^{2}}{\mathrm{~mol} \cdot \mathrm{b}}\right)}{\left(0.082054 \frac{\mathrm{L} \cdot \mathrm{atm}}{\mathrm{mol} \cdot \mathrm{K}}\right) \cdot(298.15 \mathrm{~K})} \cdot\left(\frac{1 \mathrm{~L}}{1000 \mathrm{~cm}^{3}}\right)=2.4616 \times 10^{-5} \frac{\mathrm{atoms}}{\mathrm{b} \cdot \mathrm{cm}} \\
\rho=\frac{n}{V} \cdot \frac{M}{N_{A}}=2.4616 \times 10^{-5} \frac{\mathrm{atoms}}{\mathrm{b} \cdot \mathrm{cm}} \cdot \frac{4.002602 \frac{\mathrm{g}}{\mathrm{mol}}}{\left(0.60221 \frac{\mathrm{atoms} \cdot \mathrm{cm}^{2}}{\mathrm{~mol} \cdot \mathrm{b}}\right)}=1.6361 \times 10^{-4} \frac{\mathrm{g}}{\mathrm{cm}^{3}}
\end{gathered}
$$


NEA/NSC/DOC(2006)1

Gas Cooled (Thermal) Reactor - GCR

HTTR-GCR-RESR-002

CRIT-REAC-RRATE

\subsubsection{Temperature Data}

The benchmark model temperature is $300 \mathrm{~K}$.

\subsubsection{Experimental and Benchmark-Model $\mathbf{k}_{\text {eff }}$ and / or Subcritical Parameters}

The experimental $\mathrm{k}_{\mathrm{eff}}$ was approximately at unity, made to delayed critical. A comprehensive bias assessment could not be performed; therefore, the experimental $\mathrm{k}_{\text {eff }}$ values were adjusted only for the bias incurred by removing the instrumentation in the core (Table 3.1). Furthermore, the uncertainty in the benchmark models (Tables 2.64 through 2.68) is the same as the uncertainty evaluated for the experimental, as the bias uncertainty for instrumentation has already been included. The benchmark eigenvalues for the annular HTTR core loadings are shown in Table 3.12.

Table 3.12. HTTR Benchmark Values.

\begin{tabular}{||c|c|c|c|c|c||}
\hline Case & Fuel Columns & Control $^{(\mathrm{a})}$ & $\mathrm{k}_{\mathrm{eff}}$ & $-\sigma$ & $+\sigma$ \\
\hline \hline 1 & 19 & $\mathrm{C}$ & 1.0048 & 0.0103 & 0.0100 \\
2 & 21 & $\mathrm{FS}$ & 1.0040 & 0.0100 & 0.0092 \\
3 & 24 & $\mathrm{FS}$ & 1.0035 & 0.0078 & 0.0084 \\
4 & 24 & $\mathrm{~F} 23$ & 1.0032 & 0.0080 & 0.0074 \\
5 & 27 & $\mathrm{FS}$ & 1.0029 & 0.0068 & 0.0075 \\
\hline
\end{tabular}

(a) $\mathrm{C}=$ criticality obtained using central control rod only. $\mathrm{FS}=$ flat standard pattern where $\mathrm{C}, \mathrm{R} 1$, and R2 CRs were inserted into the core at the same levels while R3 CRs were fully withdrawn. F23 = only R2 and R3 CRs were used for control while C and R1 CRs were fully withdrawn.

\subsection{Benchmark-Model Specifications for Buckling and Extrapolation-Length Measurements}

Buckling and extrapolation length measurements were not made.

\subsection{Benchmark-Model Specifications for Spectral Characteristics Measurements}

Spectral characteristics measurements were not made.

\subsection{Benchmark-Model Specifications for Reactivity Effects Measurements}

Benchmark specifications for the excess reactivity measurements pertaining to the annular core configurations are provided in Section 3.4 of HTTR-GCR-RESR-001.

\subsection{Benchmark-Model Specifications for Reactivity Coefficient Measurements}

Reactivity coefficient measurements were not made. 
NEA/NSC/DOC(2006)1

Gas Cooled (Thermal) Reactor - GCR

HTTR-GCR-RESR-002

CRIT-REAC-RRATE

\subsection{Benchmark-Model Specifications for Kinetics Measurements}

Kinetics measurements were not made.

\subsection{Benchmark-Model Specifications for Reaction-Rate Distribution Measurements}

\subsubsection{Description of the Benchmark Model Simplifications}

The simplifications of the benchmark model for determination of the axial neutron fission reaction-rate in the instrumentation columns of the HTTR (described in Section 1.7) are identical to those of the critical annular 24-fuel-column core configurations 3 and 4 described in Section 3.1.1.

\subsubsection{Dimensions}

The dimensions of the benchmark model for determination of the axial neutron reaction-rate in the instrumentation columns of the HTTR are identical to those of the critical annular 24-fuel-column core configurations 3 and 4 described in Section 3.1.2.

The axial neutron fission reaction-rate in the instrumentation columns is calculated by taking the benchmark model of the fully-loaded 30-fuel-column core and superimposing a flux tally over one of the instrumentation column positions: E05, E13, or E21. The flux is computed for 6.15-cm radius discs with a thickness of $1 \mathrm{~cm}$ located at the center of one instrumentation channel in each instrumentation column (see Figure 3.30). A total of $522 \mathrm{~cm}$, representing the total height of the core fuel and reflector blocks, was modeled. The (x, y) coordinates used for columns E05, E13, and E21, are (114.6005, 72.4), (5.4, 135.447), and (-120,63.04693), respectively, where the origin is located at the radial center of the core.

The F4 flux tally is used in MCNP, which determines the flux across a cell volume by tabulating the average track length of the neutrons. ${ }^{\text {a }}$ The tally is then modified by a tally multiplier card, Fm, that accounts for the total fission cross section of ${ }^{235} \mathrm{U}$, the fissile material in the fission chambers, to obtain the neutron reaction-rate in each instrumentation column.

The calculated neutron fission reaction rates are obtained by taking the variance-weighted average of results obtained using six variations of the input deck (Appendix A.1) with different random number seeds and tallies of the neutron reaction rate (Appendix A.3). This approach was used to reduce the statistical uncertainty in the neutron flux tallies because the relative error values obtained can underpredict the true uncertainty in the calculated neutron flux. ${ }^{b}$ Therefore, the final calculated values are obtained from a total of 18 reaction-rate tallies (6 input decks with 3 instrumentation columns each).

The average of the neutron reaction-rate in each position is taken and normalized to represent the calculated axial neutron reaction-rate profile:

$$
\phi(z)_{\text {normalized }}=\frac{\phi(z)}{\phi_{\text {maximum }}},
$$

\footnotetext{
${ }^{\text {a }}$ X-5 Monte Carlo Team, "MCNP - A General Monte Carlo N-Particle Transport Code, Version 5, Volume II: User's Guide,” LA-CP-03-0245 (April 24, 2003; revised October 2, 2005).

${ }^{b}$ F. B. Brown, “A Review of Best Practices for Monte Carlo Criticality Calculations," Proc. NCSD 2009, Richland, WA, September 13-17 (2009).
} 
NEA/NSC/DOC(2006)1

Gas Cooled (Thermal) Reactor - GCR

HTTR-GCR-RESR-002

CRIT-REAC-RRATE

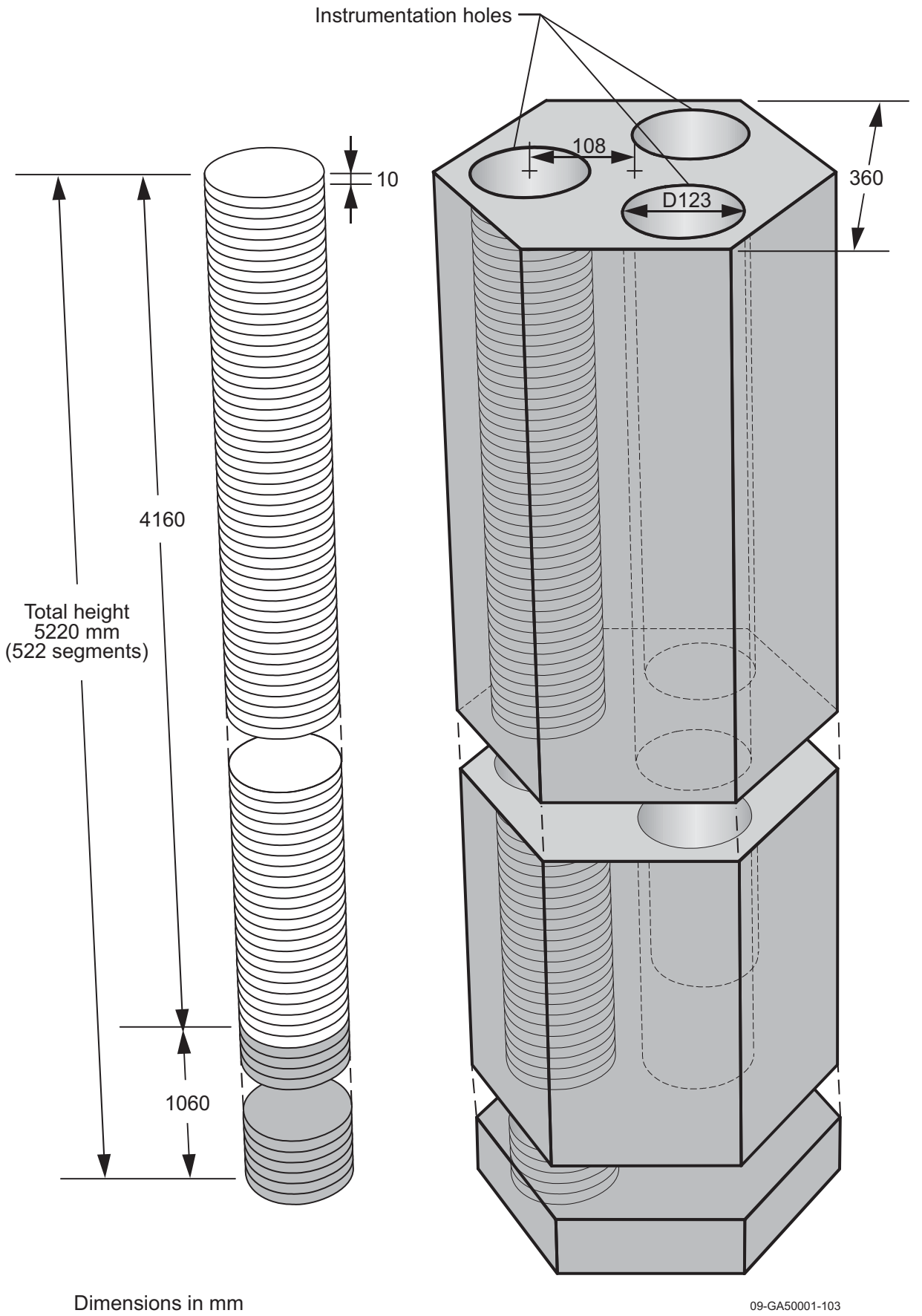

Figure 3.30. Placement of Axial Flux Tally in the Instrumentation Column

\subsubsection{Material Data}

The materials in the benchmark model for determination of the axial neutron reaction-rate in the instrumentation columns of the HTTR are identical to those in the critical annular 24-fuel-column core configurations 3 and 4 described in Section 3.1.3. 
NEA/NSC/DOC(2006)1

Gas Cooled (Thermal) Reactor - GCR

HTTR-GCR-RESR-002

CRIT-REAC-RRATE

\subsubsection{Temperature Data}

The benchmark model temperature is $300 \mathrm{~K}$.

\subsubsection{Benchmark-Model Specification for Reaction-Rate Distribution Measurements}

The expected benchmark values for the normalized axial neutron reaction-rate in the instrumentation columns of the HTTR, with their respective uncertainties (from Section 2.7.1), are shown in Table 3.13 and 3.14 for core configurations 3 and 4, respectively. The normalization is to the highest reaction-rate value, which is data point 4 at a height of 130.96 and $115.79 \mathrm{~cm}$ for core configurations 3 and 4 , respectively.

Table 3.13. Axial Neutron Fission Reaction Rate in the Instrumentation Columns of the HTTR (Configuration 3).

\begin{tabular}{||c|c|ccc|c||}
\hline $\begin{array}{c}\text { Data } \\
\text { Point }\end{array}$ & $\begin{array}{c}\text { Height } \\
(\mathrm{cm})\end{array}$ & $\begin{array}{c}\text { Normalized } \\
\text { Benchmark } \\
\text { Reaction Rate }\end{array}$ & \pm & $1 \sigma$ & $1 \sigma(\%)$ \\
\hline \hline 1 & 18.89 & 0.6946 & \pm & 0.0199 & 2.87 \\
2 & 27.86 & 0.7304 & \pm & 0.0198 & 2.70 \\
3 & 86.07 & 0.9555 & \pm & 0.0173 & 1.81 \\
4 & 130.96 & 1.0000 & \pm & 0.0166 & 1.66 \\
5 & 137.15 & 0.9854 & \pm & 0.0169 & 1.71 \\
6 & 143.96 & 0.9750 & \pm & 0.0170 & 1.75 \\
7 & 202.48 & 0.6735 & \pm & 0.0200 & 2.97 \\
8 & 260.99 & 0.2818 & \pm & 0.0165 & 5.86 \\
9 & 318.58 & 0.1041 & \pm & 0.0095 & 9.16 \\
\hline
\end{tabular}

(a) The height is in reference to the position relative to the bottom of the fifth layer of fuel. 
NEA/NSC/DOC(2006)1

Gas Cooled (Thermal) Reactor - GCR

HTTR-GCR-RESR-002

CRIT-REAC-RRATE

Table 3.14. Axial Neutron Fission Reaction Rate in the Instrumentation Columns of the HTTR (Configuration 4).

\begin{tabular}{|c|c|ccc|c||}
\hline $\begin{array}{c}\text { Data } \\
\text { Point }\end{array}$ & $\begin{array}{c}\text { Height } \\
(\mathrm{cm})\end{array}$ & $\begin{array}{c}\text { Normalized } \\
\text { Benchmark } \\
\text { Reaction Rate }\end{array}$ & \pm & $1 \sigma$ & $1 \sigma(\%)$ \\
\hline 1 & 18.79 & 0.7030 & \pm & 0.0199 & 2.83 \\
2 & 28.40 & 0.7383 & \pm & 0.0197 & 2.67 \\
3 & 86.36 & 0.9523 & \pm & 0.0174 & 1.83 \\
4 & 115.79 & 1.0000 & \pm & 0.0166 & 1.66 \\
5 & 137.45 & 0.9749 & \pm & 0.0170 & 1.75 \\
6 & 144.57 & 0.9562 & \pm & 0.0173 & 1.81 \\
7 & 202.72 & 0.7915 & \pm & 0.0193 & 2.44 \\
8 & 260.85 & 0.5582 & \pm & 0.0201 & 3.59 \\
9 & 318.37 & 0.3312 & \pm & 0.0176 & 5.32 \\
\hline
\end{tabular}

(a) The height is in reference to the position relative to the bottom of the fifth layer of fuel.

\subsection{Benchmark-Model Specifications for Power Distribution Measurements}

Power distribution measurements were not made.

\subsection{Benchmark-Model Specifications for Isotopic Measurements}

Isotopic measurements were not made.

\subsection{Benchmark-Model Specifications for Other Miscellaneous Types of Measurements}

Other miscellaneous types of measurements were not made. 
NEA/NSC/DOC(2006)1

Gas Cooled (Thermal) Reactor - GCR

HTTR-GCR-RESR-002

CRIT-REAC-RRATE

\subsection{RESULTS OF SAMPLE CALCULATIONS}

\subsection{Results of Calculations of the Critical or Subcritical Configurations}

Random particles cannot be easily modeled in MCNP. Therefore an ordered-lattice approach for modeling the benchmark was implemented, and results are provided

The computed $\mathrm{k}_{\mathrm{eff}}$ values for the benchmark model of the annular cores were evaluated with MCNP using the ENDF/B-V.2, -VI.8, and -VII.0, JEFF-3.1, and JENDL-3.3 cross section libraries. All benchmark model calculations are compared against the expected benchmark value reported in Section 3.1.5. The total uncertainty in the expected value of $\mathrm{k}_{\mathrm{eff}}$ is taken from Section 2.1.7. The JENDL-3.3 analysis was performed with the inclusion of ENDF/B-VII.0 thermal neutron scattering data because it was not included in the JENDL-3.3 library. Thermal neutron scattering, or $S(\alpha, \beta)$, adjusts the neutron cross sections for neutron upscatter at thermal energies and provides scattering data for elements bound within specific materials. The $\mathrm{k}_{\text {eff }}$ values were also calculated using ENDF/B-VII.0 and MCNPX. The MCNP5 calculations were performed with 1,050 generations (skipping the first 50) and 50,000 neutrons per generation.

It is currently difficult to obtain the necessary information to further improve the confidence in the benchmark model and effectively reduce the overall uncertainty; the necessary data is proprietary and its released is being restricted, because the benchmark configuration of the HTTR core is the same that is currently in operation. Once this information is made available, the HTTR benchmark can be adjusted as appropriate.

\subsubsection{Ordered TRISO Lattice within the Fuel Compacts}

The TRISO particles are modeled in rectangular lattices with the dimensions of $0.106 \mathrm{~cm}$ (length) $\times$ $0.106 \mathrm{~cm}$ (width) $\times 0.1 \mathrm{~cm}$ (height) to generate a volumetric packing fraction of $30 \%$ (not including the graphite overcoat) when only complete particles are placed within the compact. A cross-sectional view of the TRISO lattice block is shown in Figure 4.1. The graphite overcoat isn't completely represented in the lattice.

A horizontal cross section of the compacts is shown in Figure 4.2. As can be seen, selective placement of TRISO particles was necessary to conserve the fuel rod mass of $188.58 \mathrm{~g}$. For the current configuration, 12,987 TRISO particles are present within a standard fuel compact; this value is slightly less than the reported value of approximately 13,000.

The effective multiplication factor for configurations 1 through 5 are shown in Table 4.1 through 4.5, respectively. Calculated values of $k_{\text {eff }}$ differ from the benchmark model values by between 1.4 to $2.7 \%$. Reevaluation of the HTTR model as additional information becomes available might improve the quality of this benchmark. The benchmark models are most sensitive to graphite impurities, and graphite cross section data may also contributed to the bias. 
NEA/NSC/DOC(2006)1

Gas Cooled (Thermal) Reactor - GCR

HTTR-GCR-RESR-002

CRIT-REAC-RRATE

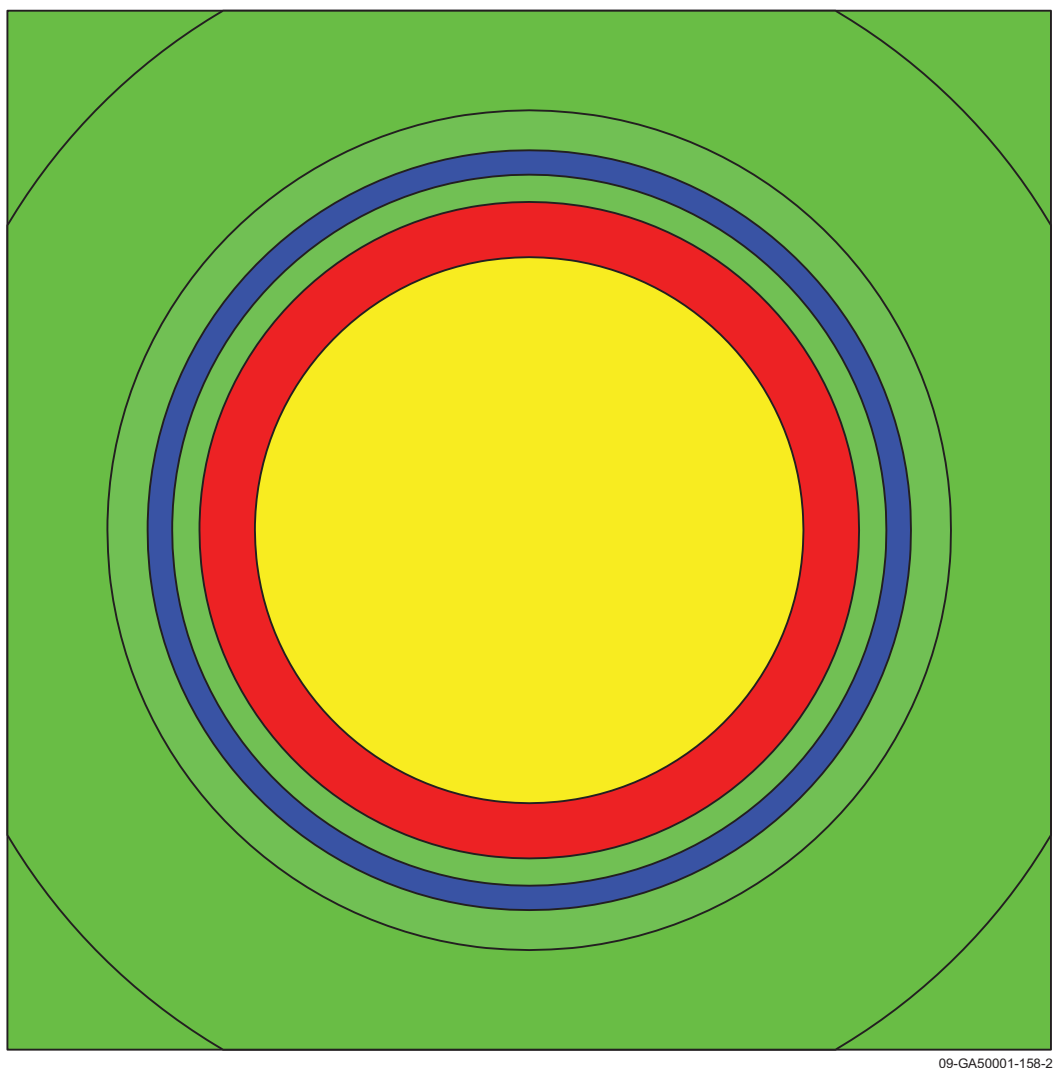

Figure 4.1. MCNP TRISO Lattice Unit Cell.

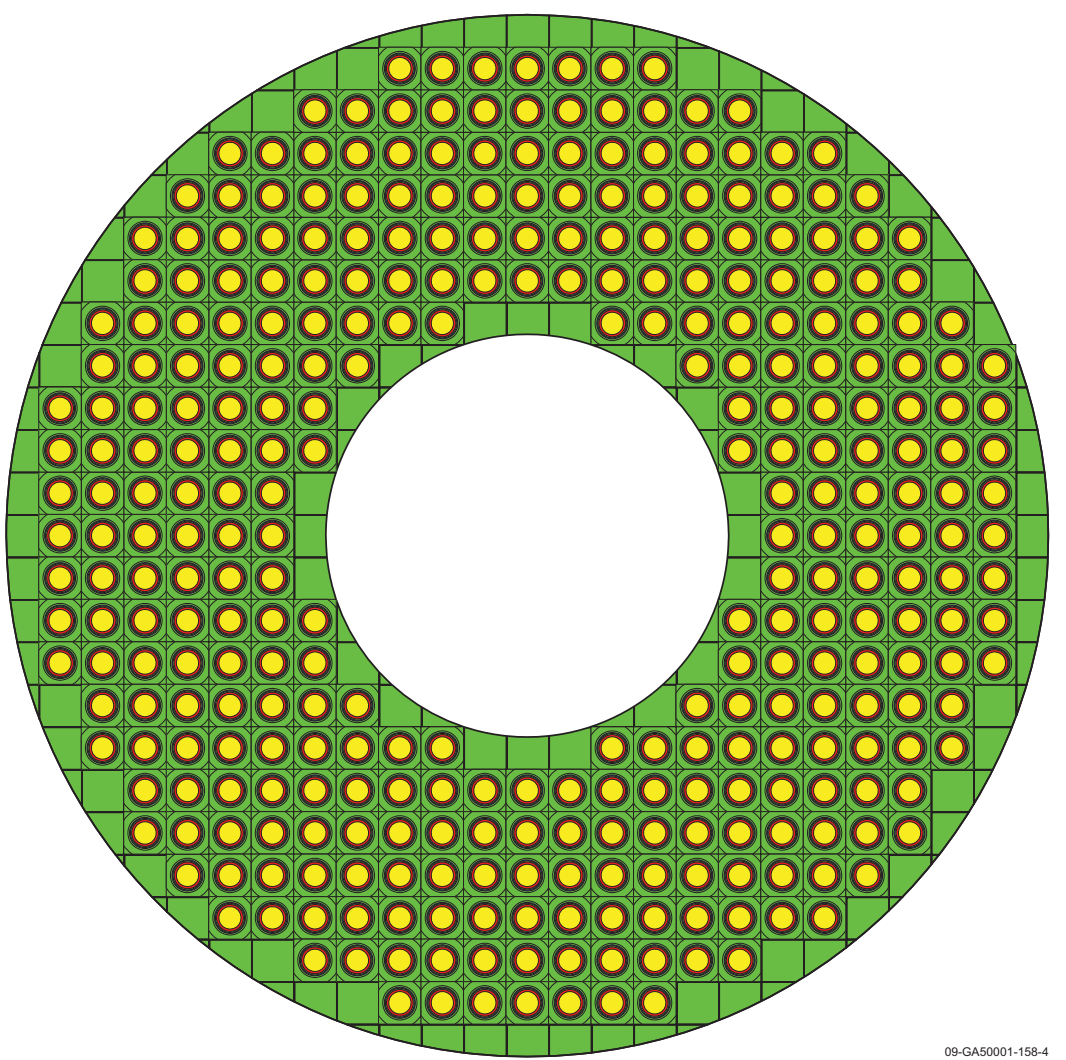

Figure 4.2. MCNP Ordered TRISO Lattice within the Fuel Compacts. 
NEA/NSC/DOC(2006)1

Gas Cooled (Thermal) Reactor - GCR

HTTR-GCR-RESR-002

CRIT-REAC-RRATE

Table 4.1. Final Results for the HTTR Benchmark Model Evaluation using an Ordered Lattice (Configuration 1).

\begin{tabular}{||c|ccc|c|cc|c||}
\hline $\begin{array}{c}\text { Neutron } \\
\text { Cross-Section } \\
\text { Library }\end{array}$ & \multicolumn{2}{|c|}{ Calculated } & Benchmark & \multicolumn{2}{|c||}{ Uncertainty } & (C-E)/E \\
\hline $\mathrm{k}_{\text {eff }}$ & \pm & $\sigma$ & $\mathrm{k}_{\mathrm{eff}}$ & $-\sigma(\%)$ & $+\sigma(\%)$ & $(\%)$ \\
\hline \hline ENDF/B-V.2 & 1.0250 & \pm & 0.0001 & $1.0048^{(\mathrm{a})}$ & 0.0103 & 0.0100 & 2.00 \\
ENDF/B-VI.8 & 1.0267 & \pm & 0.0001 & $1.0048^{(\mathrm{a})}$ & 0.0103 & 0.0100 & 2.17 \\
END/B-VII.0 & 1.0276 & \pm & 0.0001 & $1.0048^{(\mathrm{a})}$ & 0.0103 & 0.0100 & 2.27 \\
$\begin{array}{c}\text { JEFF-3.1 } \\
\text { JENDL-3.3 with }\end{array}$ & 1.0280 & \pm & 0.0001 & $1.0048^{(\mathrm{a})}$ & 0.0103 & 0.0100 & 2.31 \\
ENDF/B-VII.0 S $(\alpha, \beta)$ & 1.0222 & \pm & 0.0001 & $1.0048^{(\mathrm{a})}$ & 0.0103 & 0.0100 & 1.73 \\
\hline $\begin{array}{c}\text { ENDF/B-VII.0 } \\
\text { (MCNPX) }\end{array}$ & 1.0273 & \pm & 0.0001 & $1.0048^{(\mathrm{a})}$ & 0.0103 & 0.0100 & 2.24 \\
\hline \hline
\end{tabular}

(a) No biases have been currently evaluated for correcting the expected experimental $\mathrm{k}_{\text {eff }}$, besides the bias for removing the reactor instrumentation in the instrumentation columns.

Table 4.2. Final Results for the HTTR Benchmark Model Evaluation using an Ordered Lattice (Configuration 2).

\begin{tabular}{||c|ccc|c|cc|c||}
\hline $\begin{array}{c}\text { Neutron } \\
\text { Cross-Section } \\
\text { Library }\end{array}$ & \multicolumn{2}{|c|}{ Calculated } & Benchmark & \multicolumn{2}{|c||}{ Uncertainty } & (C-E)/E \\
\hline \hline ENDF/B-V.2 & 1.0268 & \pm & 0.0001 & $1.0040^{(\mathrm{a})}$ & 0.0100 & 0.0092 & 2.27 \\
ENDF/B-VI.8 & 1.0289 & \pm & 0.0001 & $1.0040^{(\mathrm{a})}$ & 0.0100 & 0.0092 & 2.48 \\
END/B-VII.0 & 1.0297 & \pm & 0.0001 & $1.0040^{(\mathrm{a})}$ & 0.0100 & 0.0092 & 2.55 \\
$\begin{array}{c}\text { JEFF-3.1 } \\
\text { JENDL-3.3 with }\end{array}$ & 1.0301 & \pm & 0.0001 & $1.0040^{(\mathrm{a})}$ & 0.0100 & 0.0092 & 2.60 \\
ENDF/B-VII.0 S $(\alpha, \beta)$ & 1.0241 & \pm & 0.0001 & $1.0040^{(\mathrm{a})}$ & 0.0100 & 0.0092 & 1.99 \\
\hline $\begin{array}{c}\text { ENDF/B-VII.0 } \\
\text { (MCNPX) }\end{array}$ & 1.0291 & \pm & 0.0001 & $1.0040^{(\mathrm{a})}$ & 0.0100 & 0.0092 & 2.50 \\
\hline \hline
\end{tabular}

(a) No biases have been currently evaluated for correcting the expected experimental $k_{\text {eff }}$, besides the bias for removing the reactor instrumentation in the instrumentation columns. 
NEA/NSC/DOC(2006)1

Gas Cooled (Thermal) Reactor - GCR

HTTR-GCR-RESR-002

CRIT-REAC-RRATE

Table 4.3. Final Results for the HTTR Benchmark Model Evaluation using an Ordered Lattice (Configuration 3).

\begin{tabular}{||c|ccc|c|cc|c||}
\hline $\begin{array}{c}\text { Neutron } \\
\text { Cross-Section } \\
\text { Library }\end{array}$ & \multicolumn{2}{|c|}{ Calculated } & Benchmark & \multicolumn{2}{|c||}{ Uncertainty } & (C-E)/E \\
\hline \hline ENDF/B-V.2 & 1.0224 & \pm & 0.0001 & $1.0035^{(\mathrm{a})}$ & 0.0078 & 0.0084 & 1.89 \\
ENDF/B-VI.8 & 1.0243 & \pm & 0.0001 & $1.0035^{(\mathrm{a})}$ & 0.0078 & 0.0084 & 2.07 \\
END/B-VII.0 & 1.0249 & \pm & 0.0001 & $1.0035^{(\mathrm{a})}$ & 0.0078 & 0.0084 & 2.13 \\
$\begin{array}{c}\text { JEFF-3.1 } \\
\text { JENDL-3.3 with }\end{array}$ & 1.0257 & \pm & 0.0001 & $1.0035^{(\mathrm{a})}$ & 0.0078 & 0.0084 & 2.21 \\
ENDF/B-VII.0 S $(\alpha, \beta)$ & 1.0198 & \pm & 0.0001 & $1.0035^{(\mathrm{a})}$ & 0.0078 & 0.0084 & 1.62 \\
\hline $\begin{array}{c}\text { ENDF/B-VII.0 } \\
\text { (MCNPX) }\end{array}$ & 1.0249 & \pm & 0.0001 & $1.0035^{(\mathrm{a})}$ & 0.0078 & 0.0084 & 2.13 \\
\hline \hline
\end{tabular}

(a) No biases have been currently evaluated for correcting the expected experimental $k_{\text {eff }}$, besides the bias for removing the reactor instrumentation in the instrumentation columns.

Table 4.4. Final Results for the HTTR Benchmark Model Evaluation using an Ordered Lattice (Configuration 4).

\begin{tabular}{||c|ccc|c|cc|c||}
\hline $\begin{array}{c}\text { Neutron } \\
\text { Cross-Section } \\
\text { Library }\end{array}$ & \multicolumn{2}{|c|}{ Calculated } & Benchmark & \multicolumn{2}{|c||}{ Uncertainty } & (C-E)/E \\
\hline \hline ENDF/B-V.2 & 1.0261 & \pm & 0.0001 & $1.0032^{(\mathrm{a})}$ & 0.0080 & 0.0074 & 2.29 \\
ENDF/B-VI.8 & 1.0284 & \pm & 0.0001 & $1.0032^{(\mathrm{a})}$ & 0.0080 & 0.0074 & 2.52 \\
END/B-VII.0 & 1.0287 & \pm & 0.0001 & $1.0032^{(\mathrm{a})}$ & 0.0080 & 0.0074 & 2.54 \\
$\begin{array}{c}\text { JEFF-3.1 } \\
\text { JENDL-3.3 with }\end{array}$ & 1.0298 & \pm & 0.0001 & $1.0032^{(\mathrm{a})}$ & 0.0080 & 0.0074 & 2.65 \\
ENDF/B-VII.0 S $(\alpha, \beta)$ & 1.0239 & \pm & 0.0001 & $1.0032^{(\mathrm{a})}$ & 0.0080 & 0.0074 & 2.07 \\
\hline $\begin{array}{c}\text { ENDF/B-VII.0 } \\
\text { (MCNPX) }\end{array}$ & 1.0287 & \pm & 0.0001 & $1.0032^{(\mathrm{a})}$ & 0.0080 & 0.0074 & 2.55 \\
\hline \hline
\end{tabular}

(a) No biases have been currently evaluated for correcting the expected experimental $\mathrm{k}_{\mathrm{eff}}$, besides the bias for removing the reactor instrumentation in the instrumentation columns. 
NEA/NSC/DOC(2006)1

Gas Cooled (Thermal) Reactor - GCR

HTTR-GCR-RESR-002

CRIT-REAC-RRATE

Table 4.5. Final Results for the HTTR Benchmark Model Evaluation using an Ordered Lattice (Configuration 5).

\begin{tabular}{||c|ccc|c|cc|c||}
\hline $\begin{array}{c}\text { Neutron } \\
\text { Cross-Section } \\
\text { Library }\end{array}$ & \multicolumn{2}{|c|}{ Calculated } & Benchmark & \multicolumn{2}{|c||}{ Uncertainty } & (C-E)/E \\
\hline \hline ENDF/B-V.2 & 1.0189 & \pm & 0.0001 & $1.0029^{(\mathrm{a})}$ & 0.0068 & 0.0075 & 1.60 \\
ENDF/B-VI.8 & 1.0211 & \pm & 0.0001 & $1.0029^{(\mathrm{a})}$ & 0.0068 & 0.0075 & 1.82 \\
END/B-VII.0 & 1.0218 & \pm & 0.0001 & $1.0029^{(\mathrm{a})}$ & 0.0068 & 0.0075 & 1.88 \\
$\begin{array}{c}\text { JEFF-3.1 } \\
\text { JENDL-3.3 with }\end{array}$ & 1.0224 & \pm & 0.0001 & $1.0029^{(\mathrm{a})}$ & 0.0068 & 0.0075 & 1.94 \\
ENDF/B-VII.0 S $(\alpha, \beta)$ & 1.0167 & \pm & 0.0001 & $1.0029^{(\mathrm{a})}$ & 0.0068 & 0.0075 & 1.37 \\
\hline $\begin{array}{c}\text { ENDF/B-VII.0 } \\
\text { (MCNPX) }\end{array}$ & 1.0217 & \pm & 0.0001 & $1.0029^{(\mathrm{a})}$ & 0.0068 & 0.0075 & 1.88 \\
\hline \hline
\end{tabular}

(a) No biases have been currently evaluated for correcting the expected experimental $k_{\text {eff }}$, besides the bias for removing the reactor instrumentation in the instrumentation columns.

\subsection{Results of Buckling and Extrapolation Length Calculations}

Buckling and extrapolation length measurements were not made.

\subsection{Results of Spectral-Characteristics Calculations}

Spectral characteristics measurements were not made.

\subsection{Results of Reactivity-Effects Calculations}

Sample calculation results for the excess reactivity measurements of the annular core configurations are provided in Section 4.4.1 of HTTR-GCR-RESR-001. 
NEA/NSC/DOC(2006)1

Gas Cooled (Thermal) Reactor - GCR

HTTR-GCR-RESR-002

CRIT-REAC-RRATE

\subsection{Results of Reactivity Coefficient Calculations}

Reactivity coefficient measurements were not made.

\subsection{Results of Kinetics Parameter Calculations}

Kinetics measurements were not made.

\subsection{Results of Reaction-Rate Distribution Calculations}

\subsubsection{Axial Reaction Rate Distribution}

The benchmark model for the critical annular 24-fuel-column configurations 3 and 4 described in Section 3.1 was utilized in the analysis of the reactor physics experiments in Section 1.7. The modeling approach described in Section 4.1 applies to the analysis in this section except that all calculations were performed only using the ENDF/B-VII.0 neutron cross-section library. Computed axial neutron reaction-rates in the instrumentation columns of the HTTR, averaged and normalized from tallies across the three columns from input decks using six different random number seeds, are summarized in Tables 4.6 and 4.7 as well as depicted in Figures 4.3 and 4.4 for configurations 3 and 4, respectively. The calculated flux with uncertainty bars is shown in Figures 4.5 and 4.6, respectively.

The calculated reaction rates are renormalized such that at data point 4 , both the benchmark and calculated values are 1.0000, using Equations 4.1 and 4.2, where the normalized flux at the $i^{\text {th }}$ position, $\varphi_{i, \mathrm{n}}$, is obtained by dividing the reaction rate at that position by the maximum flux, $\varphi_{i, \max }$. Then the calculated reaction rate, subscript $\mathrm{C}$, is renormalized to the maximum reaction rate of the benchmark experiment, subscript E.

$$
\begin{gathered}
\varphi_{i, n}=\frac{\varphi_{i}}{\varphi_{i, \max }} . \\
\varphi_{i, n, C}=\frac{\varphi_{i, n, C}}{\varphi_{i, n, \max , E}} .
\end{gathered}
$$

The calculated axial neutron fission reaction-rate values appear to be in good agreement with the experimental measurements; all values are within $2 \sigma$. The values reported in the right-hand column of Tables 4.6 and 4.7 represent the difference between the calculated (C) and the expected benchmark (E) values. 
NEA/NSC/DOC(2006)1

Gas Cooled (Thermal) Reactor - GCR

HTTR-GCR-RESR-002

CRIT-REAC-RRATE

Table 4.6. Calculated Axial Neutron Fission Reaction Rate in the Instrumentation Columns of the HTTR (Configuration 3).

\begin{tabular}{|c|c|ccc|cccc|c||}
\hline $\begin{array}{c}\text { Data } \\
\text { Point }\end{array}$ & $\begin{array}{c}\text { Height } \\
(\mathrm{cm})^{(\mathrm{a})}\end{array}$ & $\begin{array}{c}\text { Benchmark } \\
\text { Flux }\end{array}$ & \pm & $1 \sigma$ & Calculated & & & & \\
Flux & \pm & $\sigma$ & $\mathrm{C} / \mathrm{E}$ \\
\hline 1 & 18.89 & 0.6946 & \pm & 0.0199 & 0.6914 & \pm & 0.0014 & 0.995 \\
2 & 27.86 & 0.7304 & \pm & 0.0198 & 0.7326 & \pm & 0.0014 & 1.003 \\
3 & 86.07 & 0.9555 & \pm & 0.0173 & 0.9650 & \pm & 0.0016 & 1.010 \\
4 & 130.96 & 1.0000 & \pm & 0.0166 & 1.0000 & \pm & 0.0016 & 1.000 \\
5 & 137.15 & 0.9854 & \pm & 0.0169 & 0.9892 & \pm & 0.0016 & 1.004 \\
6 & 143.96 & 0.9750 & \pm & 0.0170 & 0.9761 & \pm & 0.0016 & 1.001 \\
7 & 202.48 & 0.6735 & \pm & 0.0200 & 0.6894 & \pm & 0.0014 & 1.024 \\
8 & 260.99 & 0.2818 & \pm & 0.0165 & 0.3043 & \pm & 0.0009 & 1.080 \\
9 & 318.58 & 0.1041 & \pm & 0.0095 & 0.1111 & \pm & 0.0005 & 1.068 \\
\hline
\end{tabular}

(a) The height is in reference to the position relative to the bottom of the fifth layer of fuel.

Table 4.7. Calculated Axial Neutron Fission Reaction Rate in the Instrumentation Columns of the HTTR (Configuration 4).

\begin{tabular}{|c|c|ccc|ccc|c||}
\hline $\begin{array}{c}\text { Data } \\
\text { Point }\end{array}$ & $\begin{array}{c}\text { Height } \\
(\mathrm{cm})\end{array}$ & $\begin{array}{c}\text { Benchmark } \\
\text { Reaction Rate }\end{array}$ & \pm & $1 \sigma$ & $\begin{array}{c}\text { Calculated } \\
\text { Reaction Rate }\end{array}$ & \pm & $1 \sigma$ & $\mathrm{C} / \mathrm{E}$ \\
\hline \hline 1 & 18.79 & 0.7030 & \pm & 0.0199 & 0.6932 & \pm & 0.0015 & 0.986 \\
2 & 28.40 & 0.7383 & \pm & 0.0197 & 0.7380 & \pm & 0.0016 & 1.000 \\
3 & 86.36 & 0.9523 & \pm 0.0174 & 0.9625 & \pm 0.0018 & 1.011 \\
4 & 115.79 & 1.0000 & \pm 0.0166 & 1.0000 & \pm 0.0018 & 1.000 \\
5 & 137.45 & 0.9749 & \pm 0.0170 & 0.9704 & \pm 0.0018 & 0.995 \\
6 & 144.57 & 0.9562 & \pm 0.0173 & 0.9569 & \pm 0.0018 & 1.001 \\
7 & 202.72 & 0.7915 & \pm 0.0193 & 0.7905 & \pm 0.0016 & 0.999 \\
8 & 260.85 & 0.5582 & \pm 0.0201 & 0.5625 & \pm 0.0014 & 1.008 \\
9 & 318.37 & 0.3312 & \pm 0.0176 & 0.3417 & \pm 0.0011 & 1.032 \\
\hline \hline
\end{tabular}

(a) The height is in reference to the position relative to the bottom of the fifth layer of fuel. 
NEA/NSC/DOC(2006)1

Gas Cooled (Thermal) Reactor - GCR

HTTR-GCR-RESR-002

CRIT-REAC-RRATE

\section{Axial Neutron Reaction-Rate in the Instrumentation Columns of the Annular 24(FS)-Fuel-Column HTTR Core}

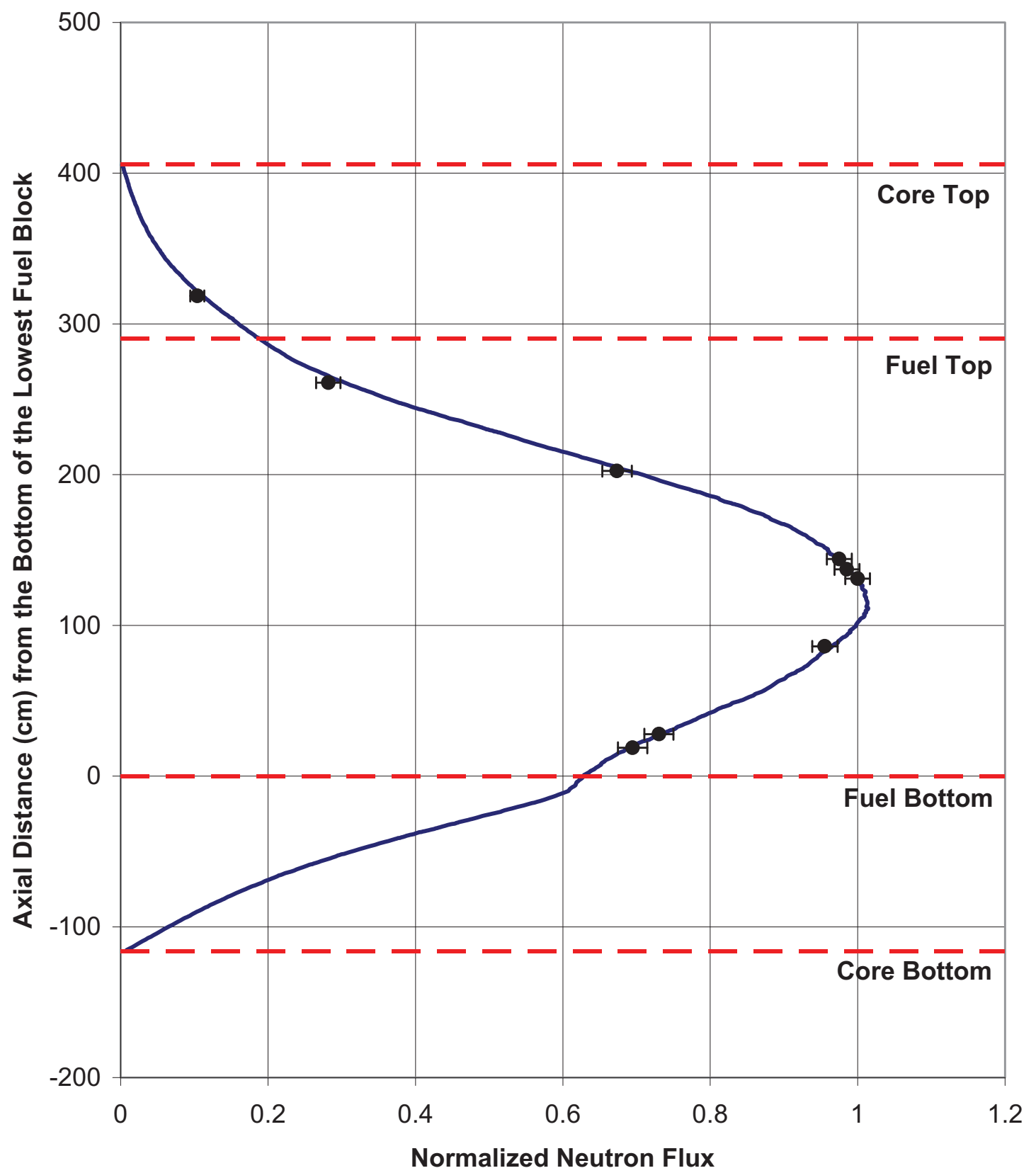

— Instrumentation Columns $\bullet$ Benchmark Values - - Core Boundaries

Figure 4.3. Calculated Axial Neutron Reaction Rate in the Instrumentation Columns of the HTTR (Configuration 3). 
NEA/NSC/DOC(2006)1

Gas Cooled (Thermal) Reactor - GCR

HTTR-GCR-RESR-002

CRIT-REAC-RRATE

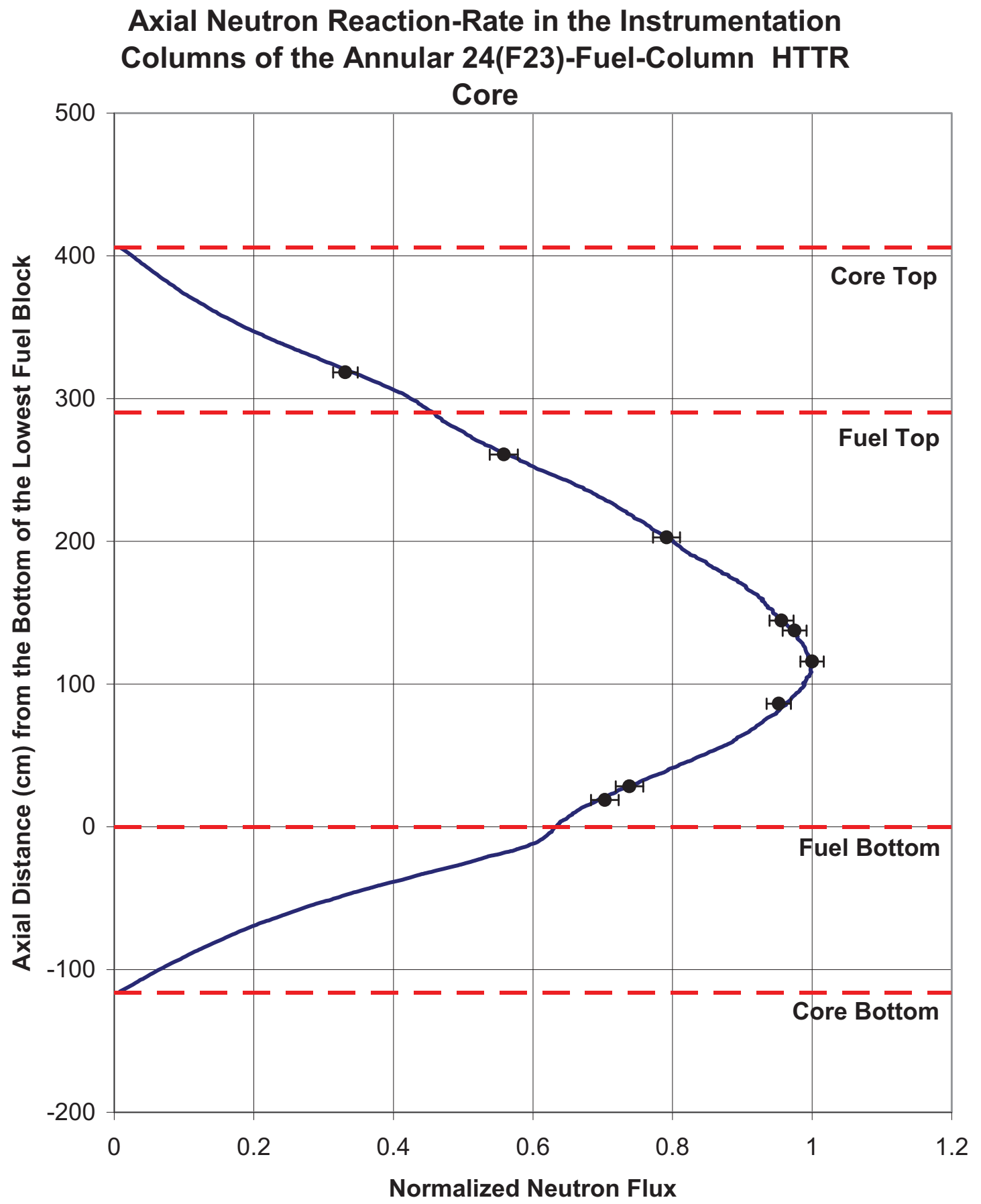

\section{Instrumentation Columns}

- Benchmark Values - - Core Boundaries

Figure 4.4. Calculated Axial Neutron Reaction Rate in the Instrumentation Columns of the HTTR (Configuration 4). 
NEA/NSC/DOC(2006)1

Gas Cooled (Thermal) Reactor - GCR

HTTR-GCR-RESR-002

CRIT-REAC-RRATE

\section{Axial Neutron Reaction-Rate in the Instrumentation Columns of the Annular 24(FS)-Fuel-Column HTTR Core}

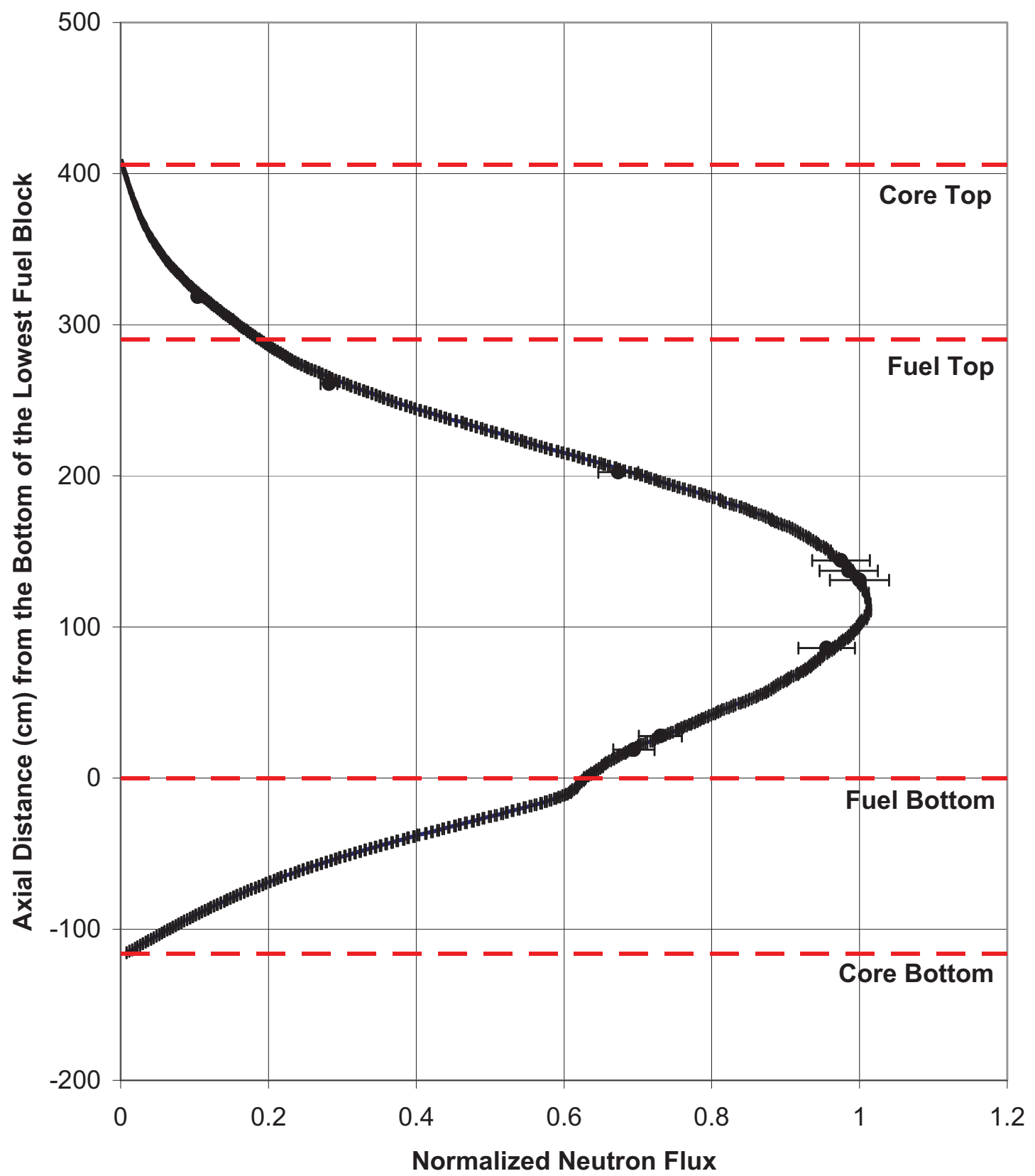

\section{Instrumentation Columns}

- Benchmark Values - - Core Boundaries

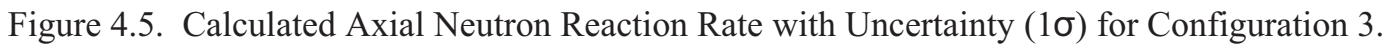


NEA/NSC/DOC(2006)1

Gas Cooled (Thermal) Reactor - GCR

HTTR-GCR-RESR-002

CRIT-REAC-RRATE

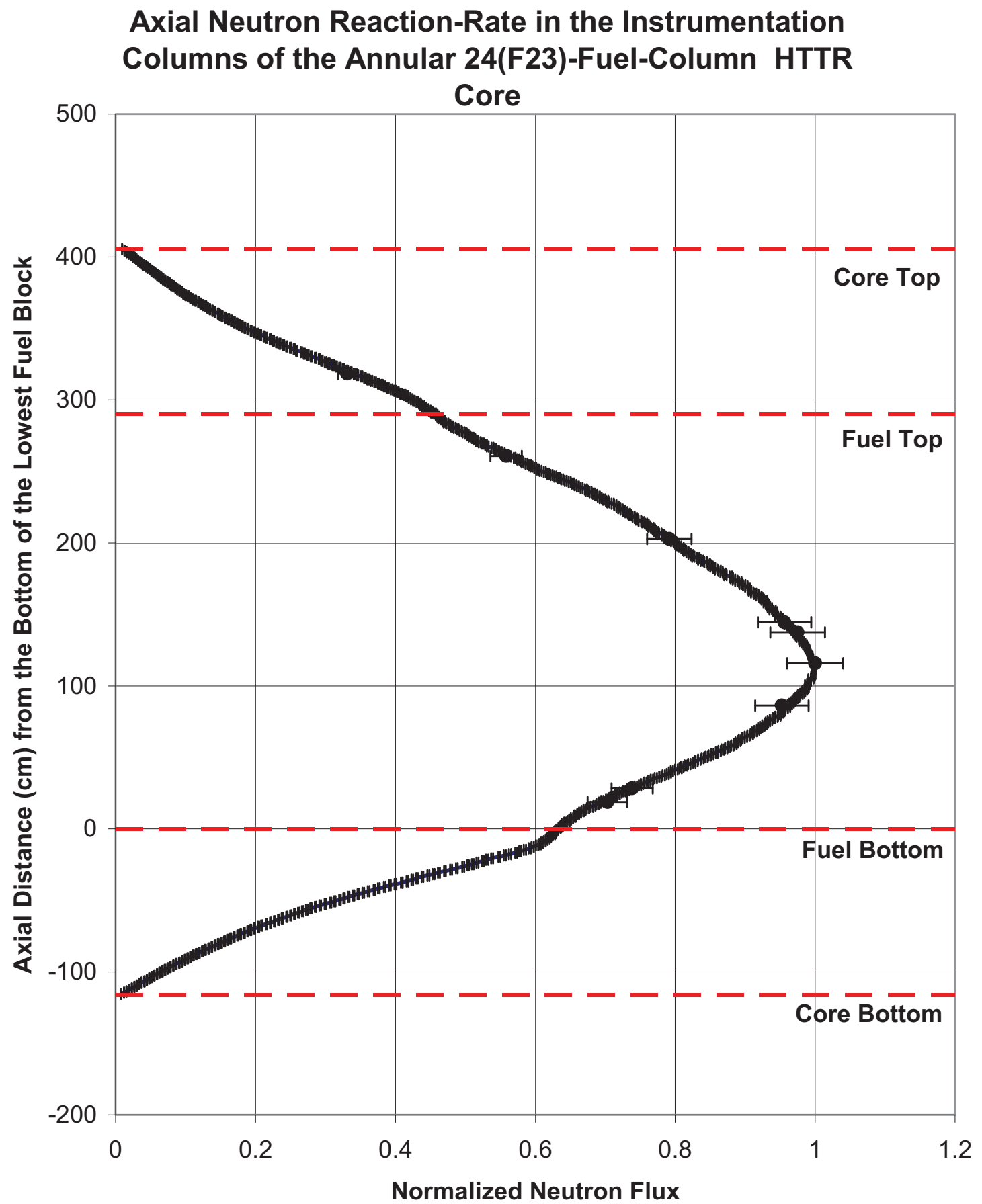

Instrumentation Columns

- Benchmark Values - - Core Boundaries

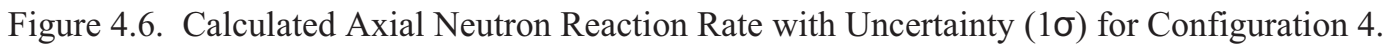




\section{NEA/NSC/DOC(2006)1 \\ Gas Cooled (Thermal) Reactor - GCR \\ HTTR-GCR-RESR-002 \\ CRIT-REAC-RRATE}

\subsection{Results of Power Distribution Calculations}

Power distribution measurements were not made.

\subsection{Results of Isotopic Calculations}

Isotopic measurements were not made.

\subsection{Results of Calculations for Other Miscellaneous Types of Measurements}

Other miscellaneous types of measurements were not made. 
NEA/NSC/DOC(2006)1

\section{Gas Cooled (Thermal) Reactor - GCR \\ HTTR-GCR-RESR-002 \\ CRIT-REAC-RRATE}

\subsection{REFERENCES}

1. N. Fujimoto, K. Yamashita, N. Nojiri, M. Takeuchi, and S. Fujisaki, "Annular Core Experiments in HTTR's Start-Up Core Physics Tests,” Nucl. Sci. Eng., 150, 310-321 (2005).

2. "Evaluation of High Temperature Gas Cooled Reactor Performance: Benchmark Analysis Related to Initial Testing of the HTTR and HTR-10," IAEA-TECDOC-1382, International Atomic Energy Agency, Vienna, November 2003.

3. S. Shiozawa, S. Fujikawa, T. Iyoku, K. Kunitomi, and Y. Tachibana, "Overview of HTTR Design Features," Nucl. Eng. Des., 233:11-21 (2004).

4. N. Nojiri, S. Shimakawa, N. Fujimoto, and M. Goto, "Characteristic Test of Initial HTTR Core," Nucl. Eng. Des., 233: 283-290 (2004). 
NEA/NSC/DOC(2006)1

Gas Cooled (Thermal) Reactor - GCR

HTTR-GCR-RESR-002

CRIT-REAC-RRATE

\section{APPENDIX A: COMPUTER CODES, CROSS SECTIONS, AND TYPICAL INPUT LISTINGS}

\section{A.1 Critical Configuration}

\section{A.1.1 Name(s) of code system(s) used.}

1. Monte Carlo n-Particle, version 5.1.40 (MCNP5)

2. Monte Carlo n-Particle Extensions, version 2.5.0 (MCNPX)

\section{A.1.2 Bibliographic references for the codes used.}

1. X-5 Monte Carlo Team, "MCNP - a General Monte Carlo n-Particle Transport Code, version 5," LA-UR-03-1987, Los Alamos National Laboratory (2003).

2. J. S. Hendricks, et al., "MCNPX Extensions," LA-UR-05-2675, Los Alamos National Laboratory (April 2005).

\section{A.1.3 Origin of cross-section data.}

The Evaluated Neutron Data File library, ENDF/B-VII.0, ${ }^{\text {a }}$ was utilized in the benchmark model analysis. Other versions, including ENDF/B-V.2 $2^{\mathrm{b}}$ and ENDF/B-VI.8, ${ }^{\mathrm{c}}$ were used with the benchmark model for a baseline comparison. The European Joint Evaluated Fission and Fusion File, JEFF-3.1 ${ }^{\mathrm{d}}$ and the Japanese Evaluated Nuclear Data Library, JENDL-3.3, ${ }^{\mathrm{e}}$ were also included for a basic evaluative comparison. Such comparisons are typical. ${ }^{\mathrm{fg}}$ The JENDL-3.3 analysis was performed with the inclusion of ENDF/BVII.0 thermal neutron scattering data because it was not included in the JENDL-3.3 library. Thermal neutron scattering, or $S(\alpha, \beta)$, adjusts the neutron cross sections for neutron upscatter at thermal energies and provides scattering data for elements bound within specific materials.

\section{A.1.4 Spectral calculations and data reduction methods used.}

Not applicable

\section{A.1.5 Number of energy groups or if continuous-energy cross sections are used in the different phases of the calculation.}

Continuous-energy cross sections

\footnotetext{
${ }^{a}$ M. B. Chadwick, et al., "ENDF/B-VII.0: Next Generation Evaluated Nuclear Data Library for Nuclear Science and Technology," Nucl. Data Sheets, 107: 2931-3060 (2006).

${ }^{\mathrm{b}}$ R. Kinsey, Ed., ENDF/B Summary Documentation, BNL-NCS-17542 (ENDF-201), $3^{\text {rd }}$ ed., Brookhaven National Laboratory (1979).

${ }^{c}$ H. D. Lemmel, P. K. McLaughlin, and V. G. Pronyaev, "ENDF/B-VI Release 8 (Last Release of ENDF/B-VI) the U.S. Evaluated Nuclear Data Library for Neutron Reaction Data," IAEA-NDS-100 Rev. 11, International Atomic Energy Agency, Vienna (November 2001).

d A. Koning, R. Forrest, M. Kellett, R. Mills, H. Henriksson, and Y. Rugama, "The JEFF-3.1 Nuclear Data Library," JEFF Report 21, Organisation for Economic Co-operation and Development, Paris (2006).

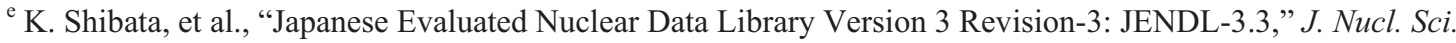
Tech., 39(11): 1125-1136 (November 2002).

${ }^{\mathrm{f}}$ A. C. Kahler, "Monte Carlo Eigenvalue Calculations withENDF/B-VI.8, JEFF-3.0, and JENDL-3.3 Cross Sections for a Selection of International Criticality Safety Benchmark Evaluation Project Handbook Benchmarks," Nucl. Sci. Eng., 145: 213-224 (2003).

${ }^{g}$ M. Goto, N. Nojiri, and S. Shimakawa, "Neutronics Calculations of HTTR with Several Nuclear Data Libraries," J. Nucl. Sci. Tech., 43(10): 1237-1244 (2006).
} 
NEA/NSC/DOC(2006)1

Gas Cooled (Thermal) Reactor - GCR

HTTR-GCR-RESR-002

CRIT-REAC-RRATE

\section{A.1.6 Component calculations.}

- Type of cell calculation - Reactor core and reflectors

- Geometry-Cylindrical

- Theory used - Not applicable

- Method used - Monte Carlo

- Calculation characteristics - histories/cycles/cycles skipped $=50,000 / 1,050 / 50$

continuous-energy cross sections

\section{A.1.7 Other assumptions and characteristics.}

Not applicable

\section{A.1.8 Typical input listings.}

MCNP5 and MCNPX Input Deck for the 27-fuel-column core, configuration 5, of the HTTR:

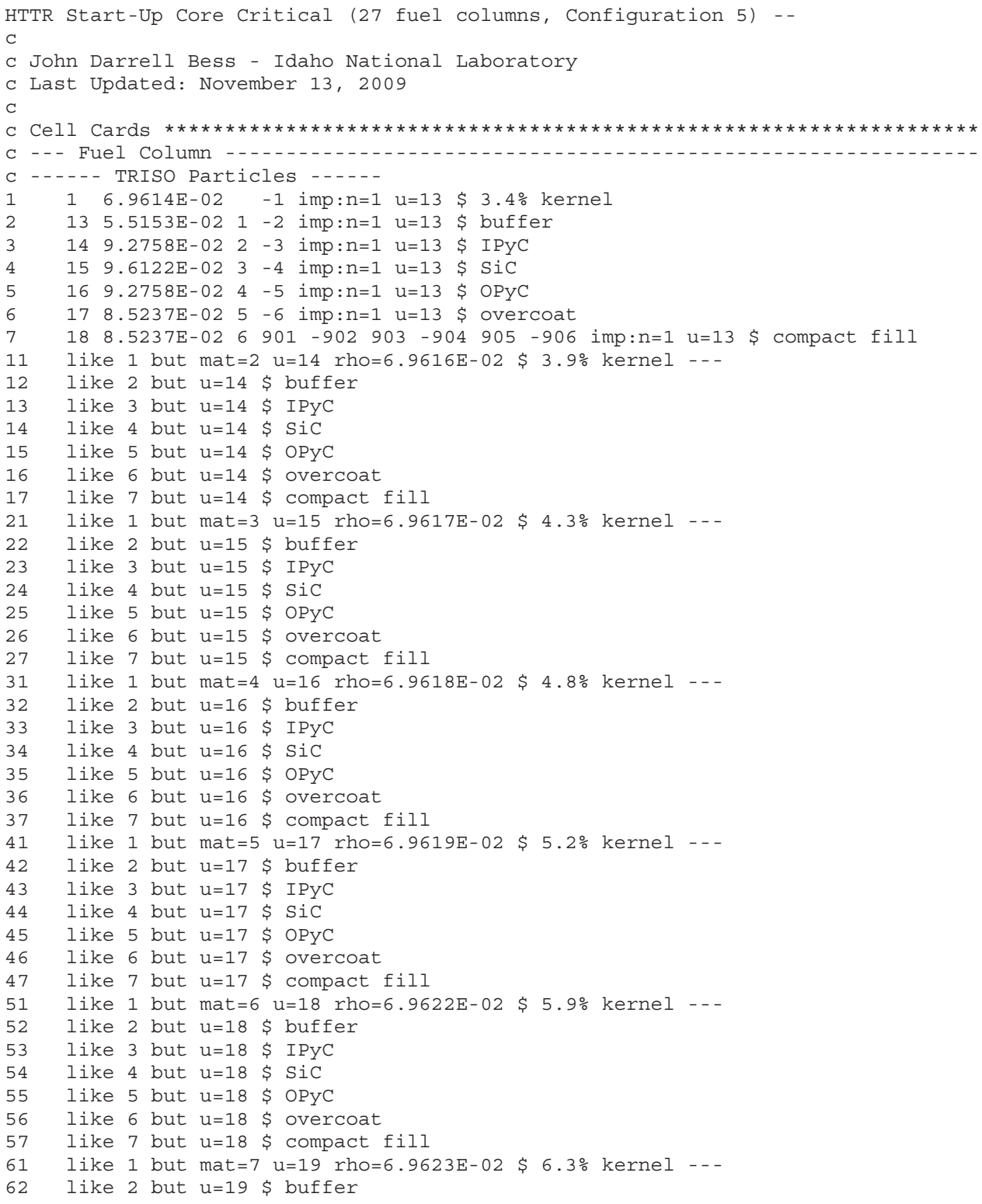

Revision: 0

Page 161 of 183

Date: March 31, 2010 
NEA/NSC/DOC(2006)1

\section{Gas Cooled (Thermal) Reactor - GCR}

\section{HTTR-GCR-RESR-002 \\ CRIT-REAC-RRATE}

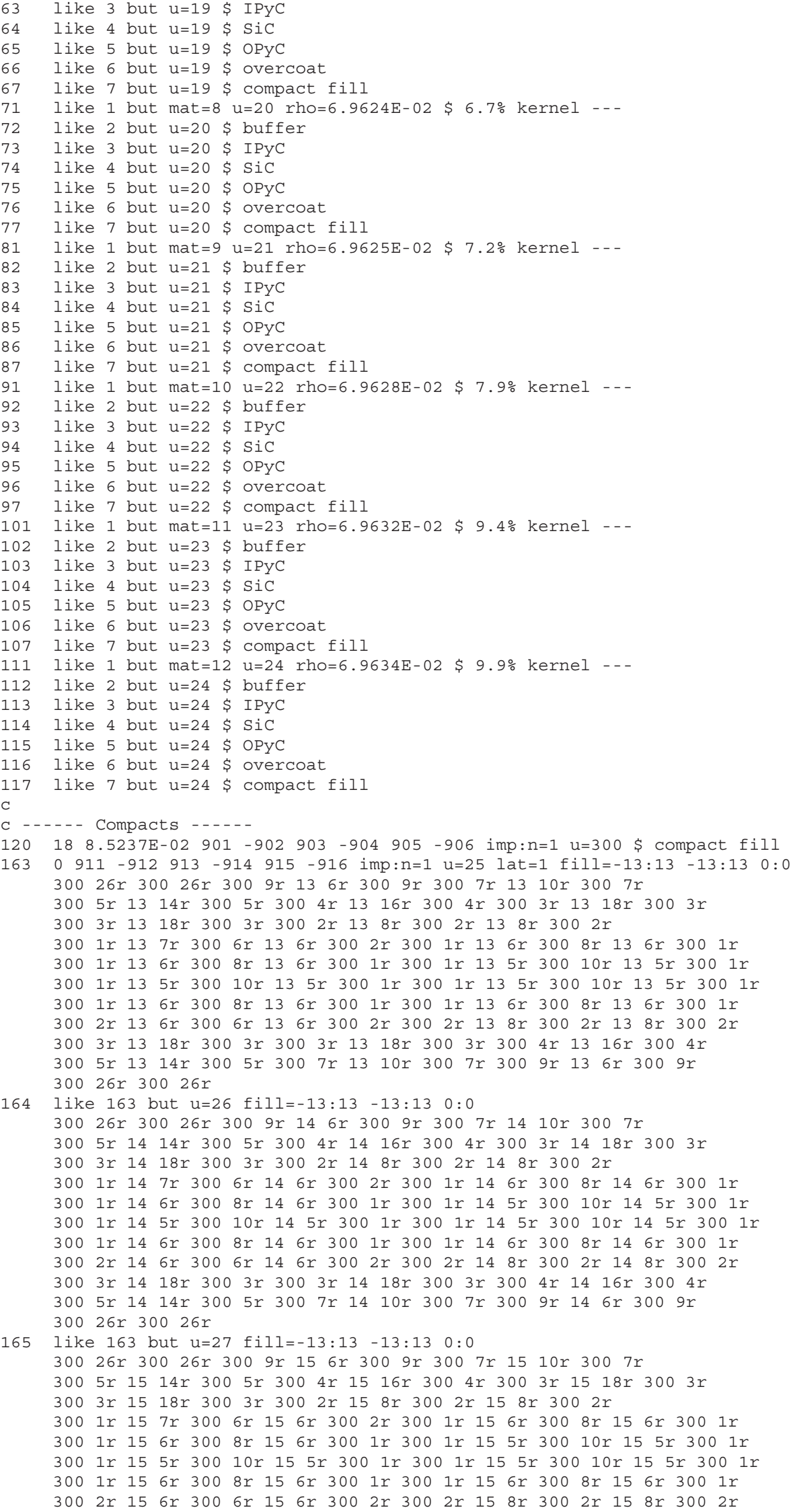

Revision: 0

Page 162 of 183

Date: March 31, 2010 
NEA/NSC/DOC(2006)1

\section{Gas Cooled (Thermal) Reactor - GCR}

\section{HTTR-GCR-RESR-002 \\ CRIT-REAC-RRATE}

$3003 r 1518 r 3003 r 300$ 3r 15 18r 300 3r 300 4r 15 16r $3004 r$ $3005 r 1514 r 300$ 5r 300 7r 15 10r 300 7r 300 9r 15 6r 300 9r $30026 r 30026 r$

166 like 163 but $u=28$ fill=-13:13 $-13: 13 \quad 0: 0$

$30026 r 30026 r 300$ 9r 16 6r 300 9r 300 7r 16 10r 300 7r

300 5r $1614 r 300$ 5r 300 4r 16 16r 300 4r 300 3r 16 18r $3003 r$

$3003 r 1618 r 3003 r 300$ 2r 16 8r 300 2r 16 8r $3002 r$

300 Ir 16 7r 300 6r 16 6r 300 2r 300 1r 16 6r 300 8r 16 6r 300 1r

300 1r 16 6r 300 8r 16 6r 300 1r 300 Ir 16 5r 300 10r 16 5r 300 ir

300 Ir 16 5r 300 10r 16 5r 300 Ir 300 Ir 16 5r $30010 r 165 r 300$ ir

300 1r 16 6r 300 8r 16 6r 300 1r 300 1r 16 6r 300 8r 16 6r 300 1r

$3002 r 16$ rr 300 rr 16 6r 300 2r 300 2r 16 8r 300 2r 16 8r 300 2r

$3003 r 1618 r 3003 r 300$ 3r 16 18r 300 3r 300 4r 16 16r $3004 r$

300 5r 16 14r 300 5r 300 7r 16 10r 300 7r 300 9r 16 6r 300 9r

$30026 r 30026 r$

167 like 163 but $u=29$ fill=-13:13 $-13: 13 \quad 0: 0$

$30026 r 30026 r 300$ 9r 17 6r 300 9r 300 7r 17 10r 300 7r

300 5r 17 14r 300 5r 300 4r 17 16r 300 4r 300 3r 17 18r 300 3r

$3003 r 1718 r 3003 r 300$ 2r 17 8r 300 2r 17 8r $3002 r$

300 Ir 17 7r 300 6r 17 6r 300 2r 300 rr 17 6r 300 8r 17 6r 300 1r

300 1r 17 6r 300 8r 17 6r 300 1r 300 Ir 17 5r 300 10r 17 5r 300 ir

300 1r 17 5r 300 10r 17 5r 300 Ir 300 Ir 17 5r $30010 r 175 r 300$ 1r

$3001 r 176 r 300$ 8r 17 6r 300 1r 300 1r 17 6r 300 8r 17 6r 300 1r

$3002 r 176 r 300$ 6r 17 6r 300 2r 300 2r 17 8r 300 2r 17 8r 300 2r

$3003 r 17$ 18r 300 3r 300 3r 17 18r 300 3r 300 4r 17 16r $3004 r$

300 5r 17 14r 300 5r 300 7r 17 10r 300 7r 300 9r 17 6r 300 9r

$30026 r 30026 r$

168 like 163 but $u=30$ fill=-13:13 $-13: 13 \quad 0: 0$

$30026 r 30026 r 300$ 9r 18 6r 300 9r 300 7r 18 10r 300 7r

$3005 r 1814 r 3005 r 300$ 4r 18 16r 300 4r 300 3r 18 18r $3003 r$

$3003 r 18$ 18r $300 \quad 3 r 300$ 2r 18 8r 300 2r 18 8r $3002 r$

300 Ir 18 7r $3006 r 18$ 6r 300 2r 300 1r 18 6r 300 8r 18 6r 300 rr

300 Ir 18 6r 300 8r 18 6r 300 1r 300 lr 18 5r 300 10r 18 5r 300 1r

300 Ir 18 5r 300 10r 18 5r 300 Ir 300 Ir 18 5r 300 10r 18 5r 300 Ir

300 Ir 18 6r 300 8r 18 6r 300 1r 300 lr 18 6r 300 8r 18 6r 300 1r

$3002 r 186 r 3006 r 18$ 6r 300 2r 300 2r 18 8r 300 2r 18 8r $3002 r$

$3003 r 1818 r 3003 r 300$ 3r 18 18r 300 3r 300 4r 18 16r $3004 r$

300 5r 18 14r 300 5r 300 7r 18 10r 300 7r 300 9r 18 6r 300 9r

$30026 r 30026 r$

169 like 163 but $u=31$ fill=-13:13 $-13: 13 \quad 0: 0$

$30026 r 30026 r 300$ 9r $196 r 300$ 9r 300 7r 19 10r 300 7r

300 5r $1914 r 300$ rr 300 4r 19 16r 300 4r 300 3r 19 18r $3003 r$

$3003 r 1918 r 3003 r 300$ 2r 19 8r 300 2r 19 8r $3002 r$

300 Ir 19 7r 300 6r $196 r 300$ 2r 300 1r 19 6r 300 8r $196 r 300$ 1r

300 1r $196 r 300$ 8r $196 r 300$ 1r 300 1r 19 5r 300 10r 19 5r 300 1r

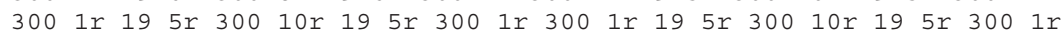

300 Ir $196 r 300$ 8r 19 6r 300 Ir 300 Ir 19 6r 300 8r $196 r 300$ 1r

300 2r $196 r 300$ 6r 19 6r 300 2r 300 2r 19 8r 300 2r 19 8r 300 2r

$3003 r 1918 r 3003 r 3003 r 1918 r 300$ 3r 300 4r $1916 r 3004 r$

300 5r $1914 r 300$ 5r 300 7r 19 10r 300 7r 300 9r $196 r 300$ 9r

$30026 r 30026 r$

180 like 163 but u=32 fill=-13:13 - $13: 13 \quad 0: 0$

$30026 r 30026 r 300$ 9r 20 6r 300 9r 300 7r 20 10r 300 7r

300 5r $2014 r 300$ 5r 300 4r 20 16r 300 4r 300 3r $2018 r 3003 r$

$3003 r 2018 r 3003 r 300$ 2r 20 8r 300 2r $208 r 3002 r$

300 Ir 20 7r $3006 r 20$ 6r 300 2r 300 1r 20 6r 300 8r 20 6r 300 1r

300 Ir $206 r 300$ 8r 20 6r 300 1r 300 1r 20 5r $30010 r 205 r 300$ Ir

300 Ir 20 5r 300 10r 20 5r 300 1r 300 Ir 20 5r 300 10r 20 5r 300 Ir

300 1r $206 r 300$ 8r $206 r 300$ 1r 300 1r 20 6r 300 8r $206 r 300$ 1r

$3002 r 206 r 3006 r 206 r 300$ 2r 300 2r 20 8r $3002 r 208 r 3002 r$

$3003 r 2018 r 3003 r 300$ 3r 20 18r 300 3r 300 4r 20 16r $3004 r$

$3005 r 2014 r 300$ 5r 300 7r 20 10r 300 7r 300 9r 20 6r 300 9r

$30026 r 30026 r$

181 like 163 but $u=33$ fill=-13:13 $-13: 13 \quad 0: 0$

$30026 r 30026 r 300$ 9r 21 6r 300 9r 300 7r 21 10r 300 7r

$3005 r 2114 r 3005 r 3004 r 21$ 16r 300 4r 300 3r $2118 r 3003 r$

$3003 r 2118 r 3003 r 300$ 2r 21 8r 300 2r $218 r 3002 r$

300 1r $217 r 3006 r 216 r 300$ 2r 300 1r $216 r 300$ 8r $216 r 3001 r$

300 1r $216 r 300$ 8r 21 6r 300 1r 300 lr 21 5r $30010 r 215 r 300$ 1r

300 1r $215 r 300$ 10r $215 r 300$ Ir 300 Ir $215 r 30010 r 215 r 300$ Ir

$3001 r 216 r 3008 r 216 r 300$ 1r 300 1r $216 r 300$ 8r $216 r 3001 r$

$3002 r 216 r 3006 r 216 r 300$ 2r 300 2r 21 8r 300 2r $218 r 3002 r$

$3003 r 2118 \times 3003 r 3003 r 21$ 18r 300 3r 300 4r 21 16r $3004 r$

300 5r 21 14r 300 5r 300 7r 21 10r 300 7r 300 9r 21 6r 300 9r

$30026 r 30026 r$

182 like 163 but $u=34$ fill=-13:13 $-13: 13 \quad 0: 0$

Revision: 0

Page 163 of 183

Date: March 31, 2010 
NEA/NSC/DOC(2006)1

\section{Gas Cooled (Thermal) Reactor - GCR \\ HTTR-GCR-RESR-002 \\ CRIT-REAC-RRATE}

$30026 r 30026 r 300$ 9r 22 6r 300 9r 300 7r 22 10r 300 7r

$3005 r 2214 r 3005 r 3004 r 22$ 16r 300 4r $3003 r 2218 r 3003 r$

$3003 r 2218 r 3003 r 300$ 2r 22 8r 300 2r 22 8r $3002 r$

300 1r 22 7r $3006 r 22$ 6r 300 2r 300 1r 22 6r 300 8r 22 6r 300 1r

300 Ir $226 r 300$ 8r 22 6r 300 1r 300 1r 22 5r 300 10r $225 r 300$ Ir

300 1r $225 r 30010 r 22$ 5r 300 1r 300 1r 22 5r $30010 r 22$ 5r 300 Ir

300 Ir $226 r 300$ 8r 22 6r 300 1r 300 1r 22 6r 300 8r 22 6r 300 1r

$3002 r 226 r 3006 r 226 r 300$ 2r 300 2r 22 8r $3002 r 228 r 3002 r$

$3003 r 2218 r 3003 r 300$ 3r 22 18r 300 3r 300 4r 22 16r $3004 r$

$3005 r 2214 r 300$ 5r 300 7r 22 10r 300 7r 300 9r 22 6r 300 9r

$30026 r 30026 r$

183 like 163 but $u=35$ fill=-13:13 $-13: 13 \quad 0: 0$

$30026 r 30026 r 300$ 9r 23 6r 300 9r 300 7r $2310 r 300$ 7r

$3005 r 2314 r 300$ 5r 300 4r 23 16r 300 4r 300 3r 23 18r $3003 r$

$3003 r 2318 r 3003 r 3002 r 23$ 8r 300 2r 23 8r $3002 r$

300 Ir $237 r 3006 r 236 r 300$ 2r 300 1r 23 6r 300 8r $236 r 300$ Ir

300 Ir 23 6r 300 8r 23 6r 300 1r 300 Ir 23 5r 300 10r 23 5r 300 1r

300 1r $235 r 300$ 10r 23 5r 300 1r 300 Ir 23 5r $30010 r 23$ 5r 300 1r

300 Ir $236 r 300$ 8r $236 r 300$ 1r 300 Ir 23 6r 300 8r $236 r 300$ 1r

$3002 r 236 r 3006 r 236 r 300$ 2r 300 2r 23 8r 300 2r 23 8r 300 2r

$3003 r 2318 r 3003 r 300$ 3r $2318 r 300$ 3r 300 4r $2316 r 3004 r$

300 5r $2314 r 300$ 5r 300 7r 23 10r 300 7r 300 9r 23 6r 300 9r

$30026 r 30026 r$

184 like 163 but $u=36$ fill=-13:13 $-13: 13 \quad 0: 0$

$30026 r 30026 r 300$ 9r 24 6r 300 9r 300 7r 24 10r 300 7r

$3005 r 2414 r 300$ 5r 300 4r 24 16r 300 4r 300 3r $2418 r 3003 r$

$3003 r 2418 r 3003 r 300$ 2r 24 8r 300 2r 24 8r $3002 r$

300 1r $247 r 300$ 6r 24 6r 300 2r 300 1r 24 6r 300 8r $246 r 300$ 1r

300 Ir $246 r 300$ 8r 24 6r 300 Ir 300 Ir 24 5r 300 10r 24 5r 300 1r

300 1r $245 r 300$ 10r 245 r 300 1r 300 1r 245 r $30010 r 245 r 300$ 1r

300 1r $246 r 300$ 8r 24 6r 300 1r 300 1r 24 6r 300 8r 24 6r 300 1r

$3002 r 246 r 3006 r 246 r 300$ 2r 300 2r 24 8r 300 2r $248 r 3002 r$

$3003 r 2418 r 3003 r 300$ 3r 24 18r 300 3r 300 4r 24 16r $3004 r$

$3005 r 2414 r 300$ 5r 300 7r 24 10r 300 7r 300 9r 24 6r 300 9r

$30026 r 30026 r$

$91630921-922923-924925-926$ imp:n=1 u=925 lat=1 fill=25

9164 like 9163 but $u=926$ fill=26

9165 like 9163 but $u=927$ fill $=27$

9166 like 9163 but $u=928$ fill $=28$

9167 like 9163 but $u=929$ fill $=29$

9168 like 9163 but $u=930 \mathrm{fill}=30$

9169 like 9163 but $u=931 \mathrm{fill}=31$

9180 like 9163 but $u=932$ fill=32

9181 like 9163 but $u=933$ fill=33

9182 like 9163 but $u=934 \mathrm{fill}=34$

9183 like 9163 but $u=935 \mathrm{fill}=35$

9184 like 9163 but $u=936$ fill=36

$185012-13$ imp: $n=1 \quad u=37 \quad \mathrm{fill}=925$

186 like 185 but $u=38$ fill $=926$

187 like 185 but $u=39$ fill $=927$

188 like 185 but $u=40 \mathrm{fill}=928$

189 like 185 but $u=41$ fill $=929$

190 like 185 but $u=42$ fill $=930$

191 like 185 but $u=43$ fill=931

192 like 185 but $u=44$ fill=932

193 like 185 but $u=45$ fill=933

194 like 185 but $u=46$ fill=934

195 like 185 but $u=47$ fill=935

196 like 185 but $u=48$ fill=936

C

C ..... Fuel Pins -....

$251272.4616 \mathrm{E}-05-12$ imp:n=1 u=37 \$ central hole

252 like 251 but $u=38$

253 like 251 but $u=39$

254 like 251 but $u=40$

255 like 251 but $\mathrm{u}=41$

256 like 251 but $u=42$

257 like 251 but $u=43$

258 like 251 but $u=44$

259 like 251 but $u=45$

260 like 251 but $u=46$

261 like 251 but $u=47$

262 like 251 but $u=48$

$263272.4616 \mathrm{E}-0513-21$ imp:n=1 u=37 \$ annulus between compact and sleeve

264 like 263 but $u=38$

265 like 263 but $u=39$

Revision: 0

Page 164 of 183

Date: March 31, 2010 
NEA/NSC/DOC(2006)1

Gas Cooled (Thermal) Reactor - GCR

HTTR-GCR-RESR-002

CRIT-REAC-RRATE

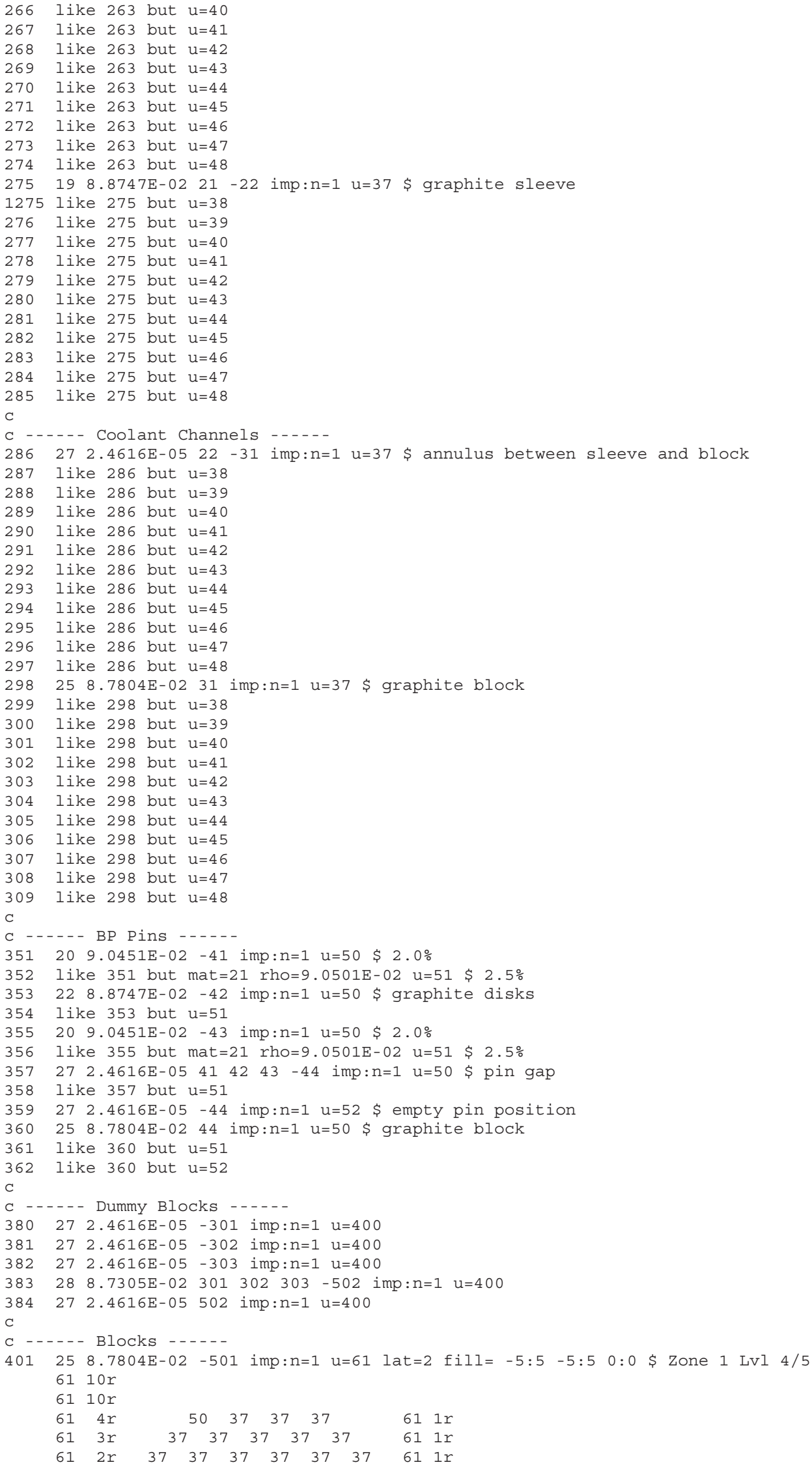

Revision: 0

Page 165 of 183

Date: March 31, 2010 
NEA/NSC/DOC(2006)1

Gas Cooled (Thermal) Reactor - GCR

\section{HTTR-GCR-RESR-002 \\ CRIT-REAC-RRATE}

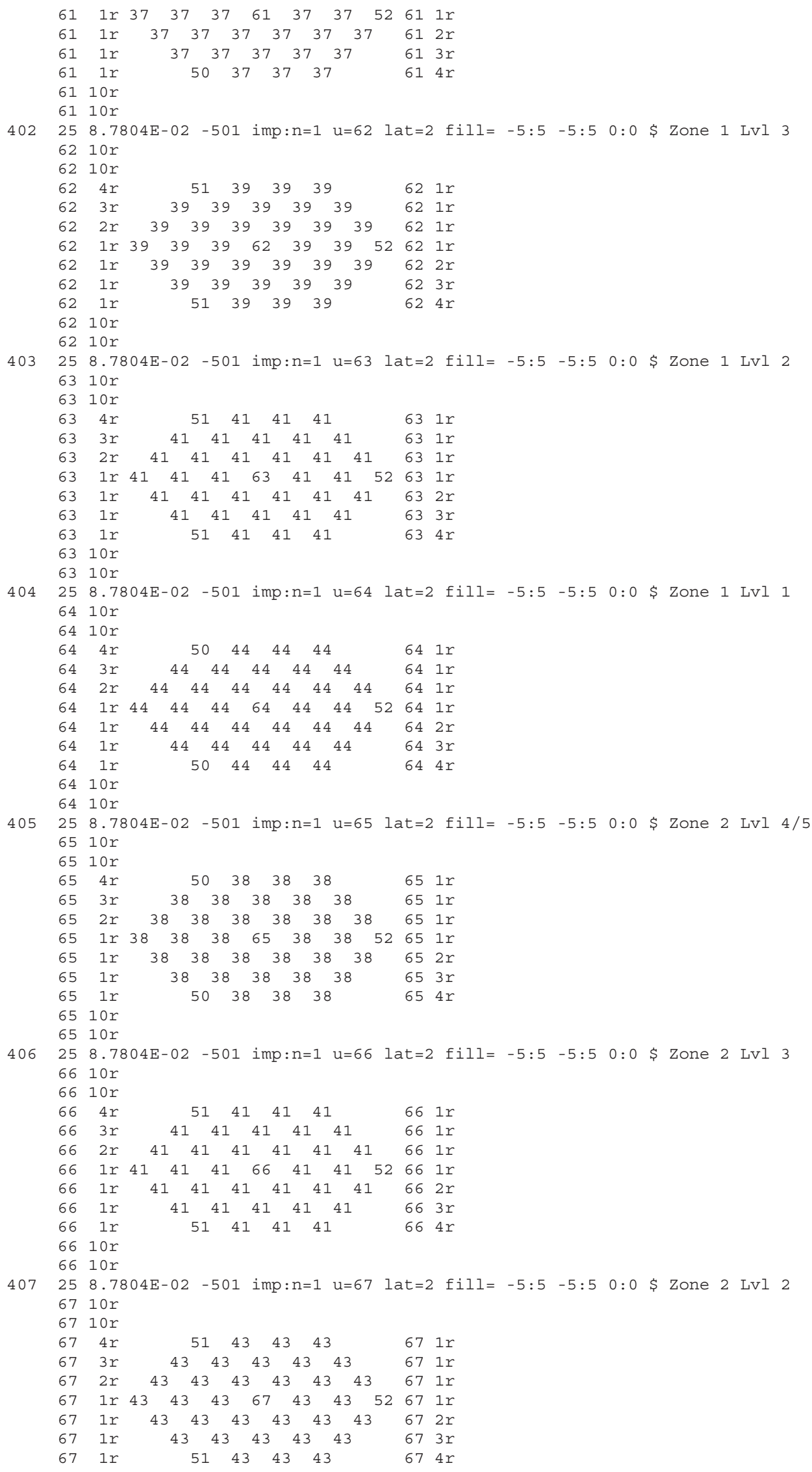

Revision: 0

Date: March 31, 2010 
NEA/NSC/DOC(2006)1

Gas Cooled (Thermal) Reactor - GCR

\section{HTTR-GCR-RESR-002 \\ CRIT-REAC-RRATE}

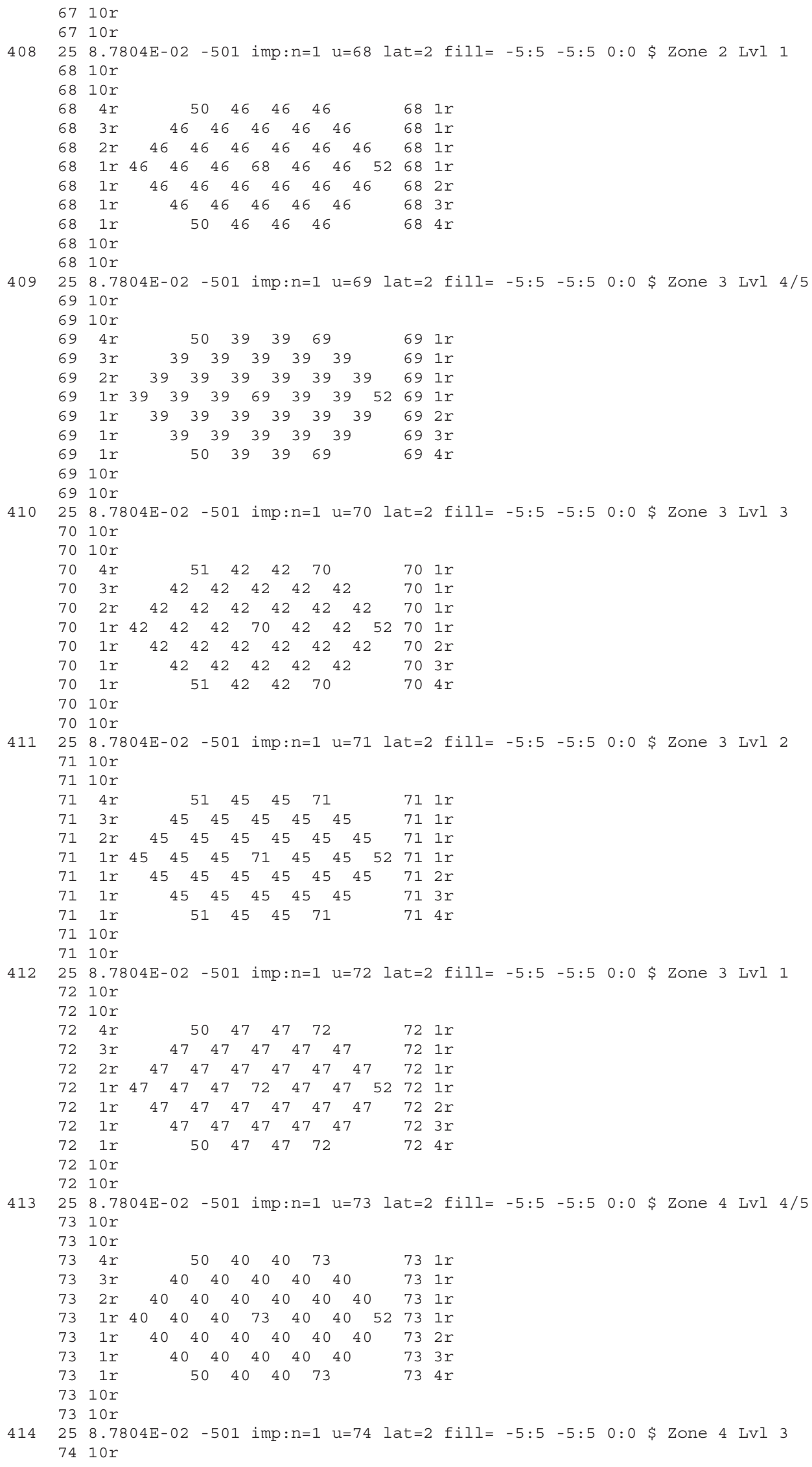

Revision: 0

Date: March 31, 2010 
NEA/NSC/DOC(2006)1

Gas Cooled (Thermal) Reactor - GCR

\section{HTTR-GCR-RESR-002 \\ CRIT-REAC-RRATE}

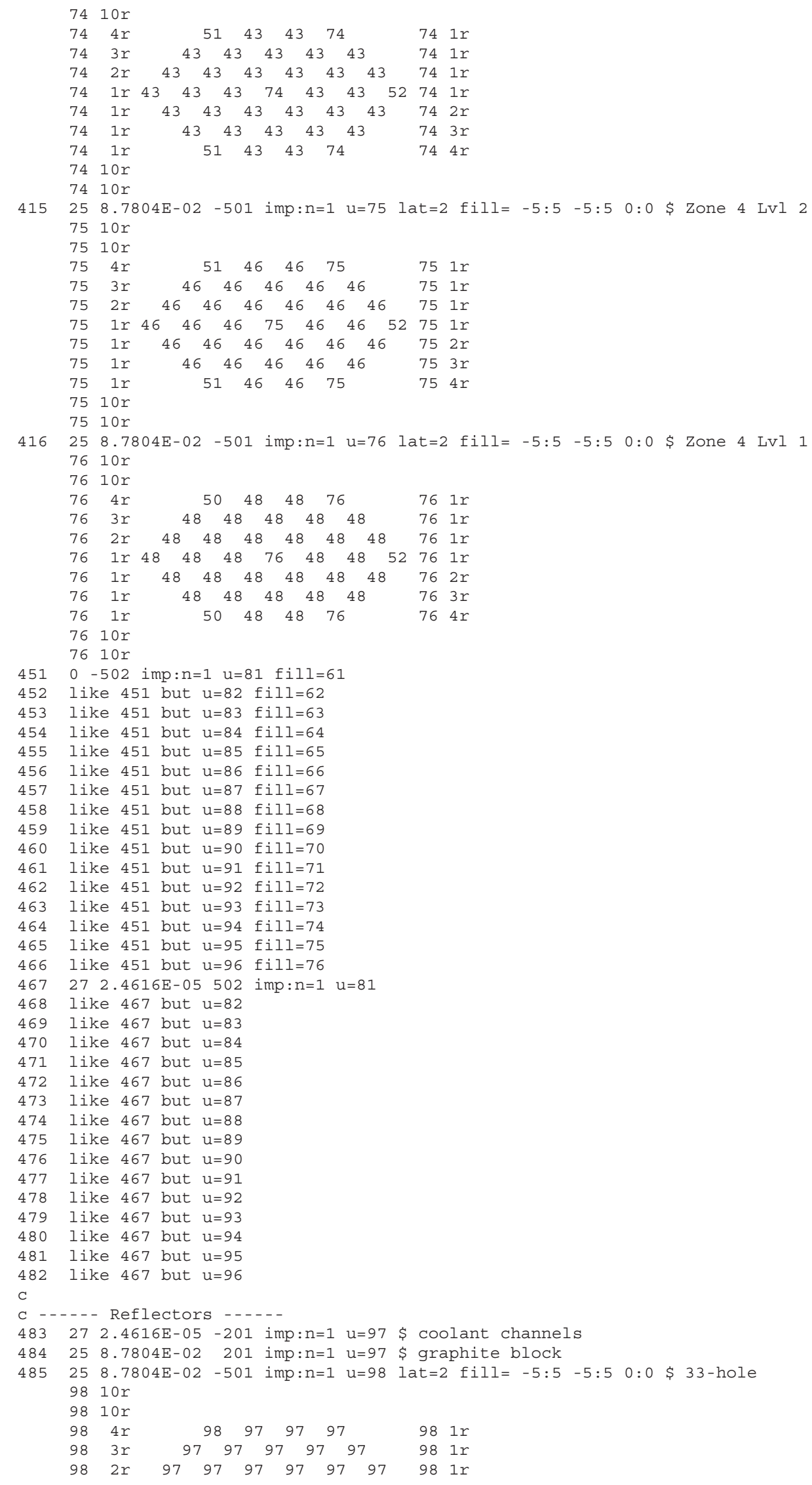

Revision: 0

Page 168 of 183

Date: March 31, 2010 
NEA/NSC/DOC(2006)1

Gas Cooled (Thermal) Reactor - GCR

\section{HTTR-GCR-RESR-002 \\ CRIT-REAC-RRATE}

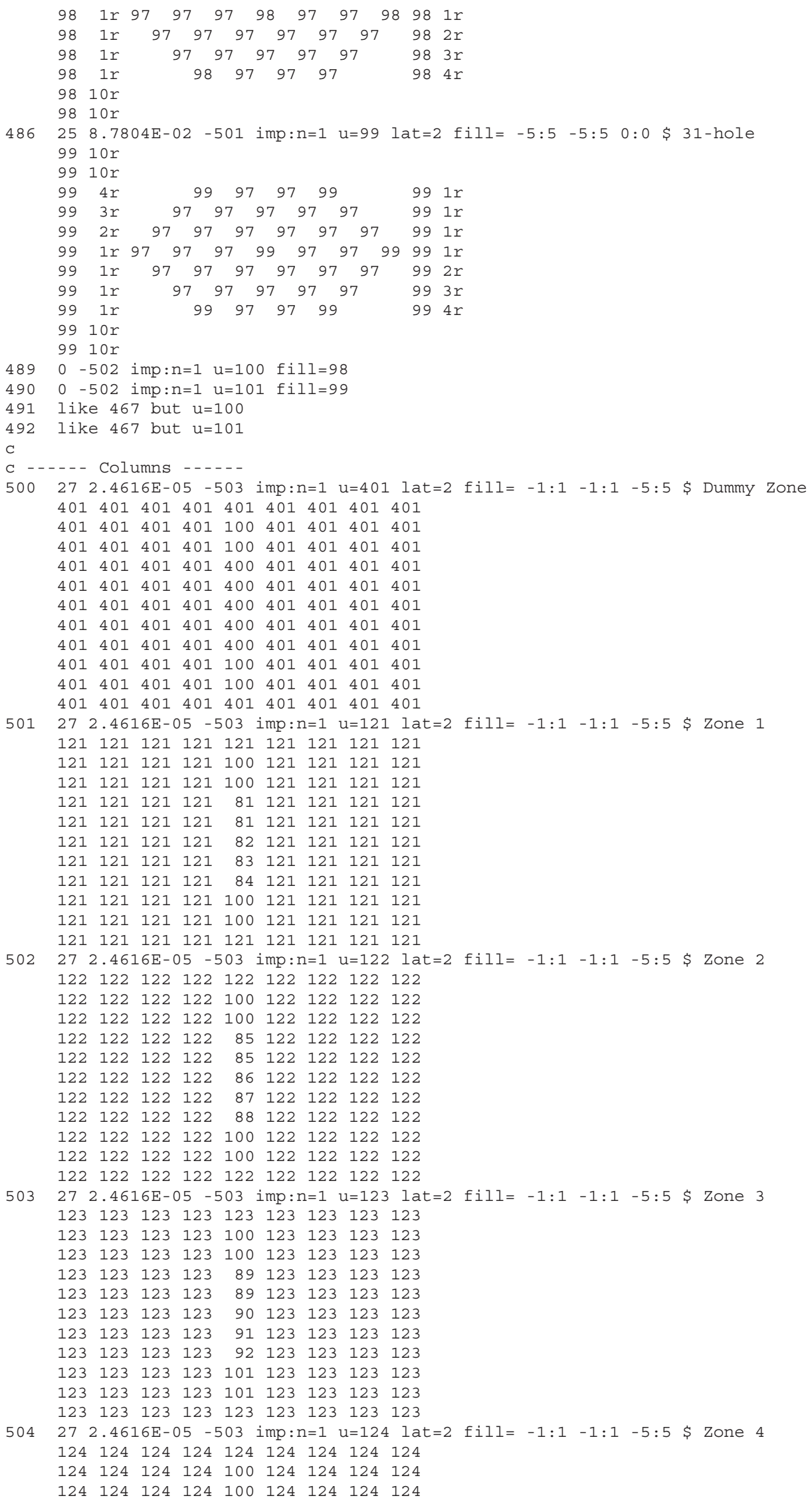

Revision: 0

Date: March 31, 2010 
NEA/NSC/DOC(2006)1

Gas Cooled (Thermal) Reactor - GCR

\section{HTTR-GCR-RESR-002 \\ CRIT-REAC-RRATE}

$\begin{array}{llllrrrrr}124 & 124 & 124 & 124 & 93 & 124 & 124 & 124 & 124 \\ 124 & 124 & 124 & 124 & 93 & 124 & 124 & 124 & 124 \\ 124 & 124 & 124 & 124 & 94 & 124 & 124 & 124 & 124 \\ 124 & 124 & 124 & 124 & 95 & 124 & 124 & 124 & 124 \\ 124 & 124 & 124 & 124 & 96 & 124 & 124 & 124 & 124 \\ 124 & 124 & 124 & 124 & 101 & 124 & 124 & 124 & 124 \\ 124 & 124 & 124 & 124 & 101 & 124 & 124 & 124 & 124 \\ 124 & 124 & 124 & 124 & 124 & 124 & 124 & 124 & 124 \\ 0 & -950 & \text { imp: } \mathrm{n}=1 & \mathrm{u}=125 & \mathrm{fill}=121 & \$ & \mathrm{~B} 04\end{array}$

Revision: 0 
NEA/NSC/DOC(2006)1

\section{Gas Cooled (Thermal) Reactor - GCR}

\section{HTTR-GCR-RESR-002 \\ CRIT-REAC-RRATE}

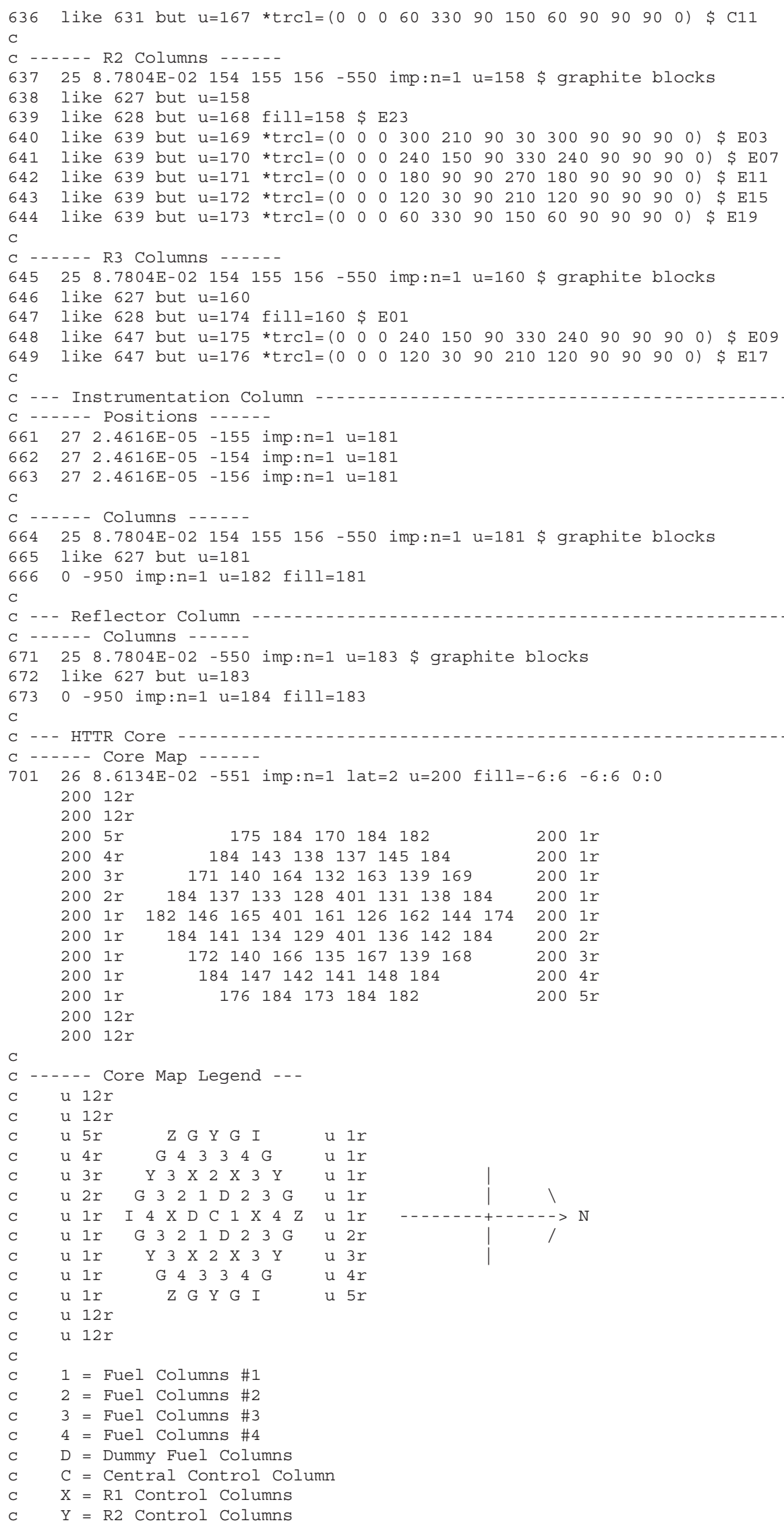

Revision: 0 
NEA/NSC/DOC(2006)1

Gas Cooled (Thermal) Reactor - GCR

\section{HTTR-GCR-RESR-002 \\ CRIT-REAC-RRATE}

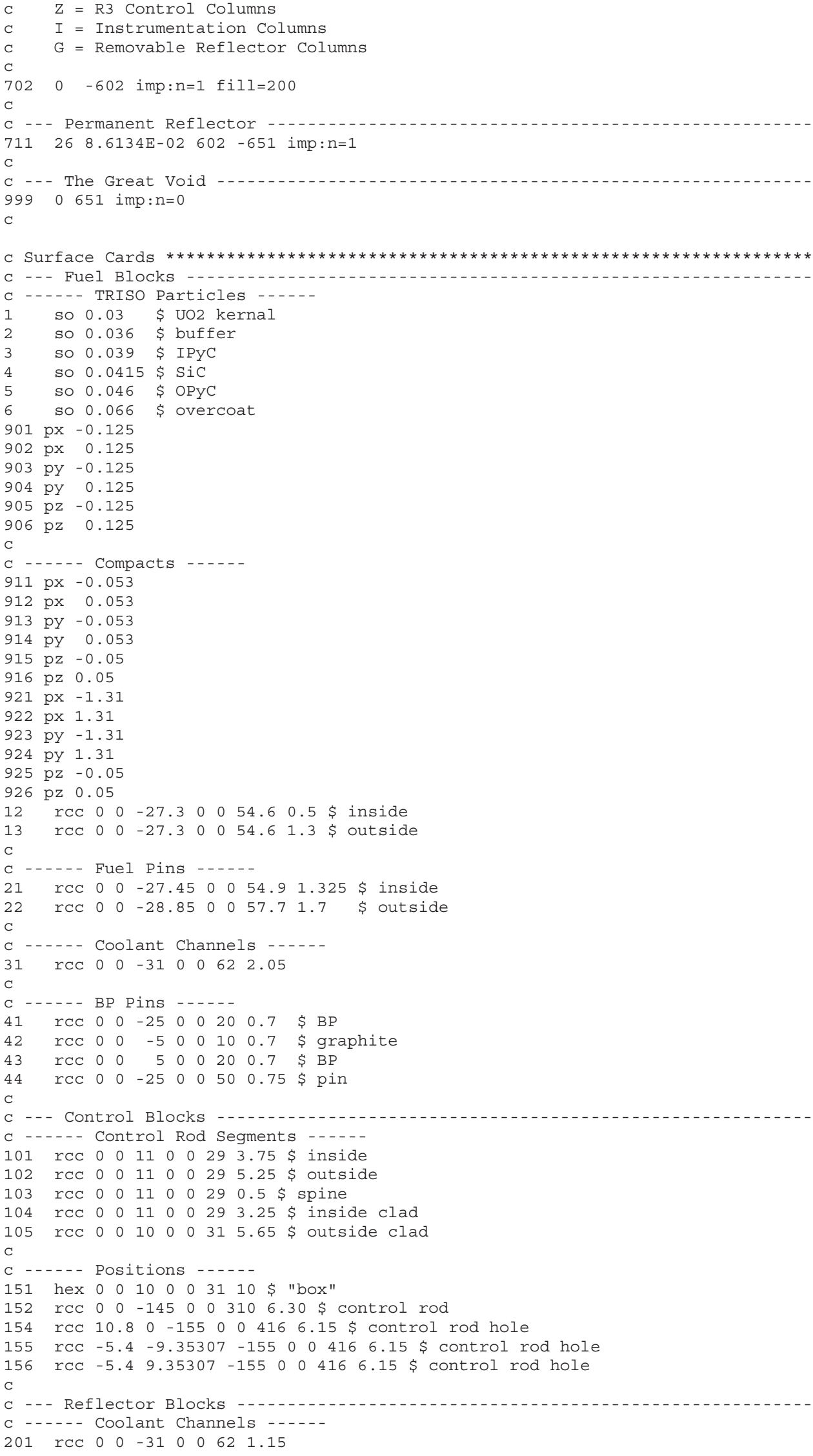

Revision: 0 
NEA/NSC/DOC(2006)1

Gas Cooled (Thermal) Reactor - GCR

\section{HTTR-GCR-RESR-002 \\ CRIT-REAC-RRATE}

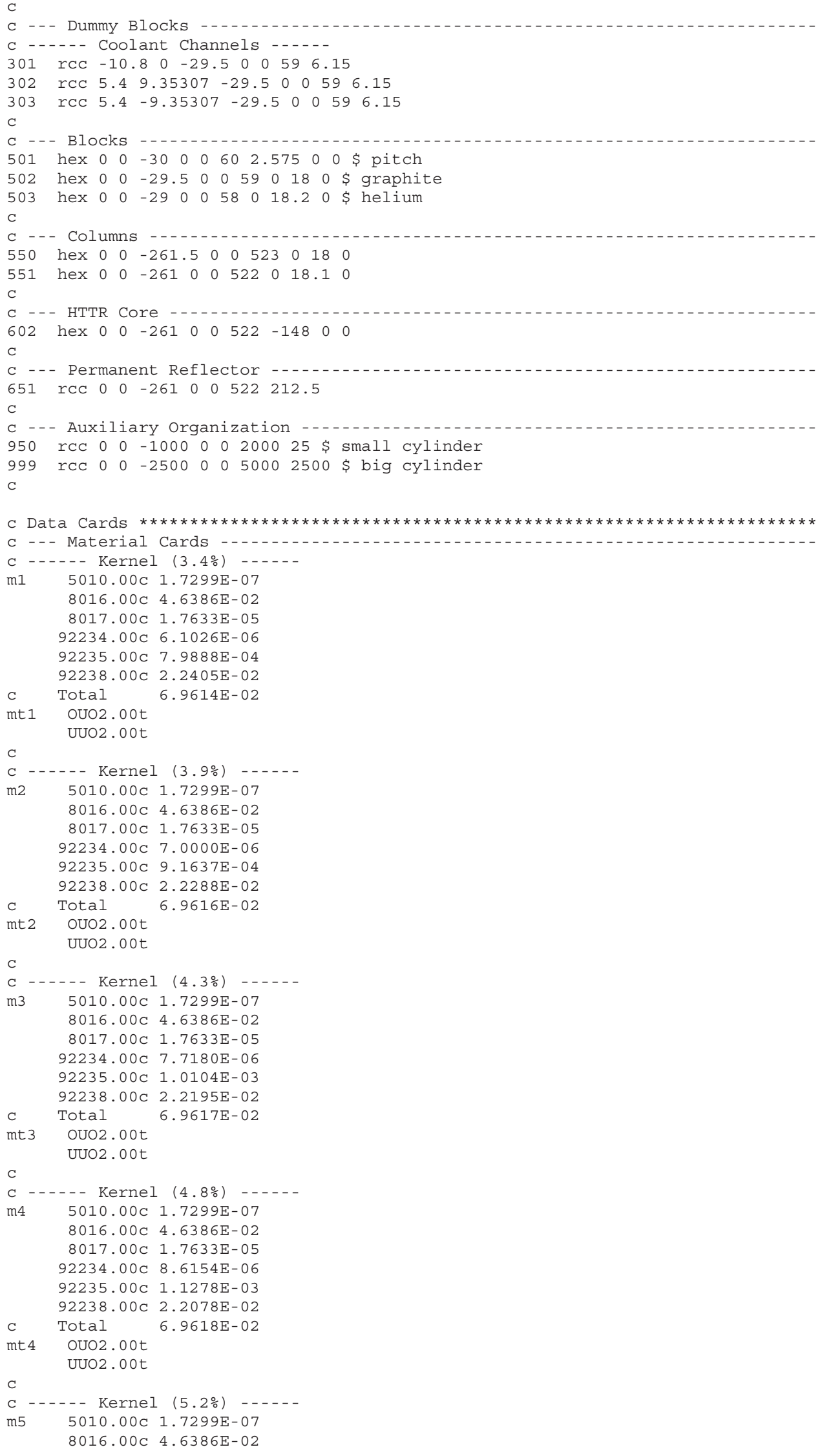

Revision: 0

Date: March 31, 2010 
NEA/NSC/DOC(2006)1

Gas Cooled (Thermal) Reactor - GCR

\section{HTTR-GCR-RESR-002 \\ CRIT-REAC-RRATE}

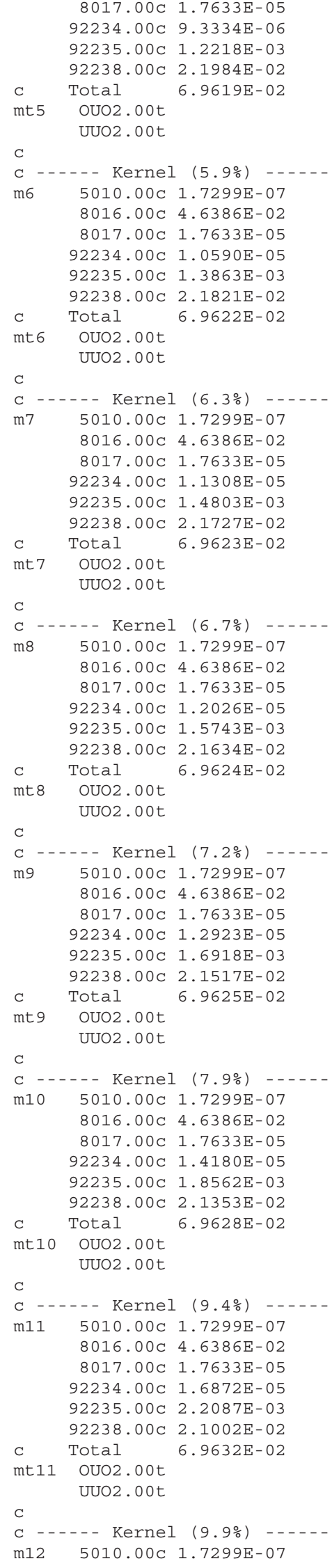

Revision: 0

Date: March 31, 2010 
NEA/NSC/DOC(2006)1

Gas Cooled (Thermal) Reactor - GCR

HTTR-GCR-RESR-002

CRIT-REAC-RRATE

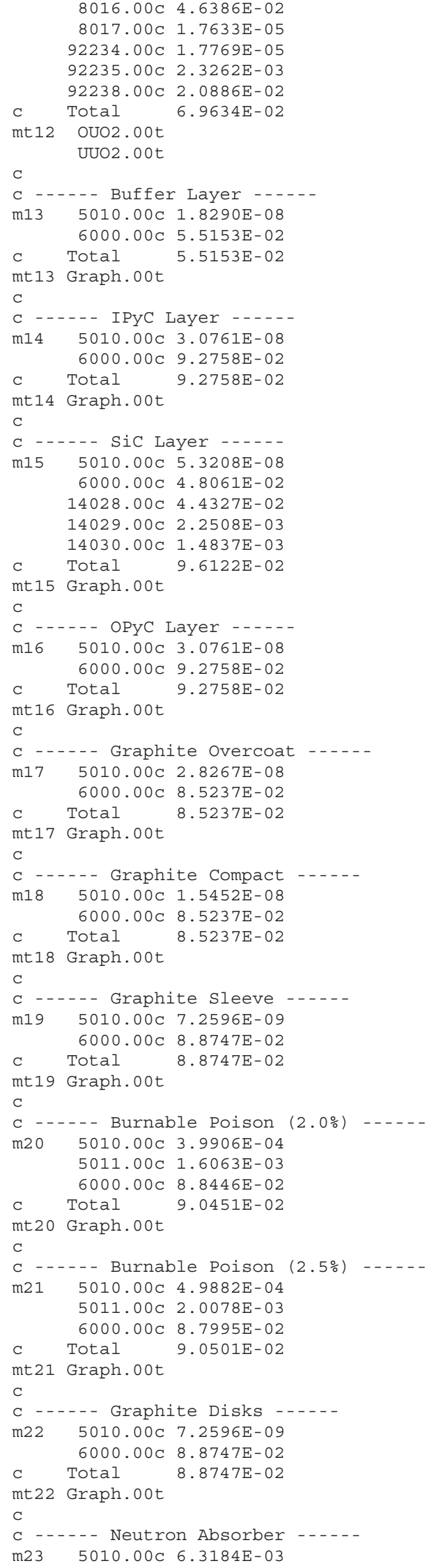

Revision: 0 
NEA/NSC/DOC(2006)1

Gas Cooled (Thermal) Reactor - GCR

\section{HTTR-GCR-RESR-002 \\ CRIT-REAC-RRATE}

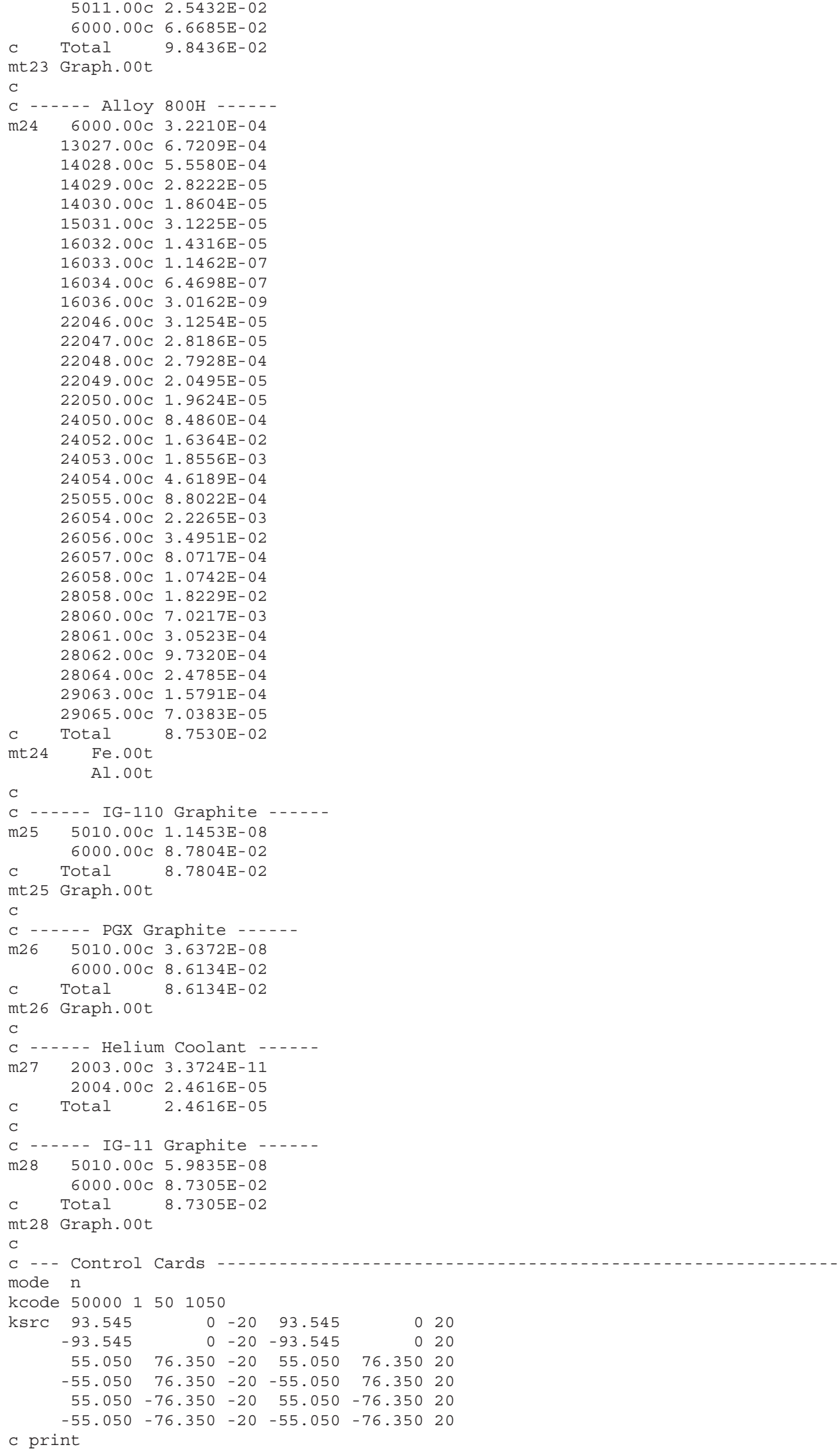

Revision: 0

Date: March 31, 2010 
NEA/NSC/DOC(2006)1

Gas Cooled (Thermal) Reactor - GCR

HTTR-GCR-RESR-002

CRIT-REAC-RRATE

\section{A.2 Buckling and Extrapolation Length Configurations}

Buckling and extrapolation length measurements were not made.

\section{A.3 Spectral-Characteristics Configurations}

Spectral characteristics measurements were not made.

\section{A.4 Reactivity-Effects Configurations}

MCNP5 Input Deck for the excess reactivity measurements of the HTTR:

The input decks from the 30-fuel-column core configuration in HTTR-GCR-RESR-001 and the 19-, 21-, 24-, and 27-fuel-column core configurations in this report are used to determine the excess reactivity of the HTTR core loading. Control rod positions are either fully withdrawn or adjusted to positions already reported in this report and HTTR-GCR-RESR-001.

\section{A.5 Reactivity Coefficient Configurations}

Reactivity coefficient measurements have not been evaluated.

\section{A.6 Kinetics Parameter Configurations}

Kinetics measurements have not been evaluated.

\section{A.7 Reaction-Rate Configurations}

MCNP5 Input Deck for the axial neutron fission reaction rate in the instrumentation columns of the HTTR:

The input deck used to determine the axial reaction rate in the instrumentation columns is that of either configuration 3 or 4 (the 24-fuel-column annular core) with the following appended coding to the end of the input deck:

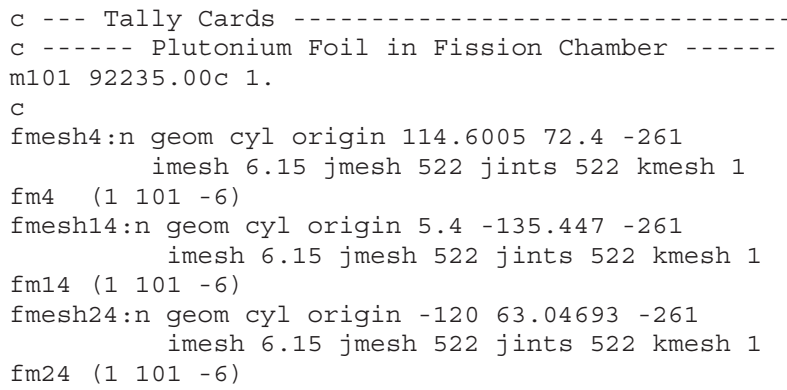


NEA/NSC/DOC(2006)1

Gas Cooled (Thermal) Reactor - GCR

HTTR-GCR-RESR-002

CRIT-REAC-RRATE

\section{A.8 Power Distribution Configuration}

Power distribution measurements were not made.

\section{A.9 Isotopic Configurations}

Isotopic measurements were not made.

\section{A.10 Configurations of Other Miscellaneous Types of Measurements}

Other miscellaneous types of measurements were not made. 
NEA/NSC/DOC(2006)1

Gas Cooled (Thermal) Reactor - GCR

HTTR-GCR-RESR-002

CRIT-REAC-RRATE

\section{APPENDIX B: CALCULATED SPECTRAL DATA}

\section{B.1 Spectral Data for the Critical and Subcritical Configurations}

Data generated in the MCNP5 output files include information regarding the energy of the average lethargy causing fission (EALF) and the percentages of fission caused by thermal, intermediate, and fast energy neutrons for each case shown in Tables 4.1 through 4.5; results are shown in Tables B.1 through B.5, respectively. There was no significant difference in the spectra when using different neutron libraries. The MCNP5 calculations were performed with 1,050 generations (skipping the first 50) and 50,000 neutrons per generation. The only significant difference is that the EALF values are slightly elevated and the neutron fission spectra is distributed slightly more in the fast energy range in Case 4. This configuration uses only the control rods in the reflector region to manage core criticality whereas the other configurations typically remove the reflector rods and maintain criticality via the core control systems. The JENDL-3.3 analysis was performed with the inclusion of ENDF/B-VII.0 thermal neutron scattering data because it was not included in the JENDL-3.3 library.

Table B.1. Spectral Data for the HTTR Benchmark Model Evaluation using an Ordered Lattice (Case 1).

\begin{tabular}{||c|c|ccc||}
\hline $\begin{array}{c}\text { Neutron } \\
\text { Cross-Section } \\
\text { Library }\end{array}$ & $\begin{array}{c}\text { EALF } \\
(\mathrm{eV})\end{array}$ & $\begin{array}{c}\text { Percentage of Neutrons Causing Fission } \\
<0.625 \mathrm{eV}\end{array}$ & $\begin{array}{c}0.625 \mathrm{eV}- \\
100 \mathrm{keV}\end{array}$ & $>100 \mathrm{keV}$ \\
\hline \hline ENDF/B-V.2 & 0.0776 & 92.36 & 6.73 & 0.91 \\
ENDF/B-VI.8 & 0.0758 & 92.53 & 6.57 & 0.90 \\
END/B-VII.0 & 0.0758 & 92.53 & 6.57 & 0.90 \\
JEFF-3.1 & 0.0755 & 92.53 & 6.57 & 0.90 \\
JENDL-3.3 with & 0.0763 & 92.48 & 6.60 & 0.92 \\
ENDF/B-VII.0 S $(\alpha, \beta)$ & \multicolumn{4}{l}{} \\
\hline
\end{tabular}

Table B.2. Spectral Data for the HTTR Benchmark Model Evaluation using an Ordered Lattice (Case 2).

\begin{tabular}{||c|c|ccc||}
\hline $\begin{array}{c}\text { Neutron } \\
\text { Cross-Section } \\
\text { Library }\end{array}$ & $\begin{array}{c}\text { EALF } \\
(\mathrm{eV})\end{array}$ & $\begin{array}{c}\text { Percentage of Neutrons Causing Fission } \\
<0.625 \mathrm{eV}\end{array}$ \\
$0.625 \mathrm{eV}-$ & $>100 \mathrm{keV}$ & $>100 \mathrm{keV}$ \\
\hline \hline ENDF/B-V.2 & 0.0774 & 92.39 & 6.71 & 0.90 \\
ENDF/B-VI.8 & 0.0757 & 92.56 & 6.55 & 0.89 \\
END/B-VII.0 & 0.0758 & 92.56 & 6.55 & 0.90 \\
JEFF-3.1 & 0.0754 & 92.56 & 6.55 & 0.89 \\
JENDL-3.3 with & 0.0761 & 92.52 & 6.57 & 0.91 \\
\hline ENDF/B-VII.0 S $(\alpha, \beta)$ &
\end{tabular}


NEA/NSC/DOC(2006)1

Gas Cooled (Thermal) Reactor - GCR

HTTR-GCR-RESR-002

CRIT-REAC-RRATE

Table B.3. Spectral Data for the HTTR Benchmark Model Evaluation using an Ordered Lattice (Case 3).

\begin{tabular}{||c|c|ccc||}
\hline $\begin{array}{c}\text { Neutron } \\
\text { Cross-Section } \\
\text { Library }\end{array}$ & $\begin{array}{c}\text { EALF } \\
(\mathrm{eV})\end{array}$ & $\begin{array}{c}\text { Percentage of Neutrons Causing Fission } \\
<0.625 \mathrm{eV} \\
0.625 \mathrm{eV}-\end{array}$ & $>100 \mathrm{keV}$ & $\mathrm{keV}$ \\
\hline \hline ENDF/B-V.2 & 0.0775 & 92.41 & 6.69 & 0.90 \\
ENDF/B-VI.8 & 0.0759 & 92.56 & 6.55 & 0.89 \\
END/B-VII.0 & 0.0759 & 92.57 & 6.54 & 0.89 \\
JEFF-3.1 & 0.0756 & 92.58 & 6.54 & 0.89 \\
JENDL-3.3 with & 0.0763 & 92.53 & 6.57 & 0.90 \\
ENDF/B-VII.0 S $(\alpha, \beta)$ & 0.073 \\
\hline
\end{tabular}

Table B.4. Spectral Data for the HTTR Benchmark Model Evaluation using an Ordered Lattice (Case 4).

\begin{tabular}{||c|c|ccc||}
\hline $\begin{array}{c}\text { Neutron } \\
\text { Cross-Section } \\
\text { Library }\end{array}$ & $\begin{array}{c}\text { EALF } \\
(\mathrm{eV})\end{array}$ & $\begin{array}{c}\text { Percentage of Neutrons Causing Fission } \\
<0.625 \mathrm{eV}\end{array}$ & $\begin{array}{c}0.625 \mathrm{eV}- \\
100 \mathrm{keV}\end{array}$ & $>100 \mathrm{keV}$ \\
\hline \hline ENDF/B-V.2 & 0.0813 & 91.98 & 7.11 & 0.91 \\
ENDF/B-VI.8 & 0.0795 & 92.15 & 6.94 & 0.90 \\
END/B-VII.0 & 0.0797 & 92.14 & 6.95 & 0.91 \\
JEFF-3.1 & 0.0792 & 92.16 & 6.94 & 0.90 \\
JENDL-3.3 with & 0.0800 & 92.11 & 6.98 & 0.92 \\
\hline
\end{tabular}

Table B.5. Spectral Data for the HTTR Benchmark Model Evaluation using an Ordered Lattice (Case 5).

\begin{tabular}{||c|c|ccc||}
\hline $\begin{array}{c}\text { Neutron } \\
\text { Cross-Section } \\
\text { Library }\end{array}$ & $\begin{array}{c}\text { EALF } \\
(\mathrm{eV})\end{array}$ & $\begin{array}{c}\text { Percentage of Neutrons Causing Fission } \\
<0.625 \mathrm{eV}\end{array}$ & $\begin{array}{c}0.625 \mathrm{eV}- \\
100 \mathrm{keV}\end{array}$ & $>100 \mathrm{keV}$ \\
\hline \hline ENDF/B-V.2 & 0.0769 & 92.52 & 6.58 & 0.90 \\
ENDF/B-VI.8 & 0.0752 & 92.69 & 6.42 & 0.89 \\
END/B-VII.0 & 0.0753 & 92.69 & 6.42 & 0.89 \\
JEFF-3.1 & 0.0749 & 92.70 & 6.42 & 0.89 \\
JENDL-3.3 with & 0.0756 & 92.66 & 6.45 & 0.90 \\
ENDF/B-VII.0 S $(\alpha, \beta)$ & \multicolumn{4}{l}{} \\
\hline
\end{tabular}


NEA/NSC/DOC(2006)1

Gas Cooled (Thermal) Reactor - GCR

HTTR-GCR-RESR-002

CRIT-REAC-RRATE

\section{APPENDIX C: DATA FROM THE $16^{\text {TH }}$ EDITION CHART OF THE NUCLIDES ${ }^{\mathrm{a}}$}

\section{C.1 Isotopic Abundances and Atomic Weights}

This evaluation incorporated atomic weights and isotopic abundances found in the $16^{\text {th }}$ edition of the Chart of the Nuclides. A list of the values used in the benchmark model or in the generation of the MCNP input deck is compiled in Table C.1.

Table C.1. Summary of Data Employed from the $16^{\text {th }} \mathrm{Ed}$. of the Chart of the Nuclides.

\begin{tabular}{||ccc||}
\hline $\begin{array}{c}\text { Isotope or } \\
\text { Element }\end{array}$ & $\begin{array}{c}\text { Atomic } \\
\text { Weight }\end{array}$ & $\begin{array}{c}\text { Isotopic } \\
\text { Abundance }\end{array}$ \\
\hline \hline $\mathrm{He}$ & 4.002602 & -- \\
${ }^{3} \mathrm{He}$ & -- & 0.000137 \\
${ }^{4} \mathrm{He}$ & -- & 99.999863 \\
\hline${ }^{10} \mathrm{~B}$ & 10.0129370 & 19.9 \\
${ }^{11} \mathrm{~B}$ & 11.0093055 & 80.1 \\
\hline $\mathrm{C}$ & 12.0107 & -- \\
\hline $\mathrm{N}$ & 14.0067 & -- \\
${ }^{14} \mathrm{~N}$ & -- & 99.632 \\
${ }^{15} \mathrm{~N}$ & -- & 0.368 \\
\hline $\mathrm{O}$ & 15.9994 & -- \\
${ }^{16} \mathrm{O}$ & -- & 99.757 \\
${ }^{17} \mathrm{O}$ & -- & 0.038 \\
${ }^{18} \mathrm{O}{ }^{(\mathrm{a})}$ & -- & 0.205 \\
\hline $\mathrm{Na}$ & 22.989770 & -- \\
\hline $\mathrm{Na}$ & 26.981538 & -- \\
\hline${ }^{16}$ & 28.0855 & -- \\
${ }^{32} \mathrm{Si}$ & -- & 92.2297 \\
${ }^{28} \mathrm{Si}$ & -- & 4.6832 \\
${ }^{36} \mathrm{~S}$ & -- & 3.0872 \\
${ }^{29} \mathrm{Si}$ & -- & -- \\
${ }^{30} \mathrm{Si}$ & -- & 04.93 \\
\hline${ }^{32} \mathrm{P}$ & 30.973761 & 4.29 \\
$\mathrm{~S}$ & 32.065 & 0.02 \\
\hline & -- & - \\
\hline & -- & - \\
\hline
\end{tabular}

${ }^{\text {a }}$ Nuclides and Isotopes: Chart of the Nuclides, $16^{\text {th }}$ edition, (2002). 
NEA/NSC/DOC(2006)1

Gas Cooled (Thermal) Reactor - GCR

HTTR-GCR-RESR-002

CRIT-REAC-RRATE

Table C.1 (cont'd.). Summary of Data Employed from the $16^{\text {th }} \mathrm{Ed}$. of the Chart of the Nuclides.

\begin{tabular}{|c|c|c|}
\hline $\begin{array}{c}\text { Isotope or } \\
\text { Element }\end{array}$ & $\begin{array}{l}\text { Atomic } \\
\text { Weight }\end{array}$ & $\begin{array}{c}\text { Isotopic } \\
\text { Abundance }\end{array}$ \\
\hline${ }^{40} \mathrm{Ca}$ & -- & 96.941 \\
\hline${ }^{42} \mathrm{Ca}$ & -- & 0.647 \\
\hline${ }^{43} \mathrm{Ca}$ & -- & 0.135 \\
\hline${ }^{44} \mathrm{Ca}$ & -- & 2.086 \\
\hline${ }^{46} \mathrm{Ca}$ & -- & 0.004 \\
\hline${ }^{48} \mathrm{Ca}$ & -- & 0.187 \\
\hline $\mathrm{Ti}$ & 47.867 & -- \\
\hline${ }^{46} \mathrm{Ti}$ & -- & 8.25 \\
\hline${ }^{47} \mathrm{Ti}$ & -- & 7.44 \\
\hline${ }^{48} \mathrm{Ti}$ & -- & 73.72 \\
\hline${ }^{49} \mathrm{Ti}$ & -- & 5.41 \\
\hline${ }^{50} \mathrm{Ti}$ & -- & 5.18 \\
\hline $\mathrm{Cr}$ & 51.9961 & -- \\
\hline${ }^{50} \mathrm{Cr}$ & -- & 4.345 \\
\hline${ }^{52} \mathrm{Cr}$ & -- & 83.789 \\
\hline${ }^{53} \mathrm{Cr}$ & -- & 9.501 \\
\hline${ }^{54} \mathrm{Cr}$ & -- & 2.365 \\
\hline $\mathrm{Mn}$ & 54.938049 & -- \\
\hline $\mathrm{Fe}$ & 55.845 & -- \\
\hline${ }^{54} \mathrm{Fe}$ & -- & 5.845 \\
\hline${ }^{56} \mathrm{Fe}$ & -- & 91.754 \\
\hline${ }^{57} \mathrm{Fe}$ & -- & 2.119 \\
\hline${ }^{58} \mathrm{Fe}$ & -- & 0.282 \\
\hline $\mathrm{Ni}$ & 58.6934 & -- \\
\hline${ }^{58} \mathrm{Ni}$ & -- & 68.0769 \\
\hline${ }^{60} \mathrm{Ni}$ & -- & 26.2231 \\
\hline${ }^{61} \mathrm{Ni}$ & -- & 1.1399 \\
\hline${ }^{62} \mathrm{Ni}$ & -- & 3.6345 \\
\hline${ }^{64} \mathrm{Ni}$ & -- & 0.9256 \\
\hline $\mathrm{Cu}$ & 63.546 & -- \\
\hline${ }^{63} \mathrm{Cu}$ & -- & 69.17 \\
\hline${ }^{65} \mathrm{Cu}$ & -- & 30.83 \\
\hline
\end{tabular}


NEA/NSC/DOC(2006)1

Gas Cooled (Thermal) Reactor - GCR

HTTR-GCR-RESR-002

CRIT-REAC-RRATE

Table C.1 (cont'd.). Summary of Data Employed from the $16^{\text {th }} \mathrm{Ed}$. of the Chart of the Nuclides.

\begin{tabular}{||ccc||}
\hline $\begin{array}{c}\text { Isotope or } \\
\text { Element }\end{array}$ & $\begin{array}{c}\text { Atomic } \\
\text { Weight }\end{array}$ & $\begin{array}{c}\text { Isotopic } \\
\text { Abundance }\end{array}$ \\
\hline \hline${ }^{234} \mathrm{U}$ & 234.040946 & $0.0055^{(\mathrm{b})}$ \\
${ }^{235} \mathrm{U}$ & 235.043923 & $0.7200^{(\mathrm{b})}$ \\
${ }^{238} \mathrm{U}$ & 238.050783 & $99.2745^{(\mathrm{b})}$ \\
\hline
\end{tabular}

(a) Neutronically, ${ }^{18} \mathrm{O}$ is treated as ${ }^{16} \mathrm{O}$.

(b) Natural isotopic abundance of $U$. 